\title{
Transport, disorder and reaction in spreading phenomena
}

\author{
Dissertation
}

\author{
zur Erlangung des Doktorgrades \\ der Mathematisch-Naturwissenschaftlichen Fakultäten \\ der Georg-August-Universität zu Göttingen
}

vorgelegt von

Vitaly Belik

aus Moskau

Göttingen, 2008 
D 7

Referent:

Prof. Dr. Theo Geisel

Koreferent:

Prof. Dr. Marcus Müller

Tag der mündlichen Prüfung: 
светлой памяти Ниониле и Прасковье

to Nionila and Praskovia, in memoriam 



\section{Contents}

1 Introduction 4

2 Fundamentals $\quad 8$

2.1 Superdiffusion . . . . . . . . . . . . . . . . . . 8

2.2 Disorder and gravity model . . . . . . . . . . . . . . . . . 12

2.3 Basic epidemiological models . . . . . . . . . . . . . . . . . . 14

2.3 .1 SI model . . . . . . . . . . . . . . . . . . . . . 15

2.3 .2 SIR model . . . . . . . . . . . . . . . . . . 16

2.3 .3 SIS model . . . . . . . . . . . . . . . . . . 17

2.4 Spatial spread of epidemics . . . . . . . . . . . . . . . . 17

3 Balancing between start and destination: superdiffusion and gravity model 22

3.1 The model . . . . . . . . . . . . . . . . . . . 22

3.1.1 Disordered environment . . . . . . . . . . . . . 24

3.1.1.1 Periodic potentials . . . . . . . . . . . . . 25

3.1.1.2 Random potentials . . . . . . . . . . . . . 26

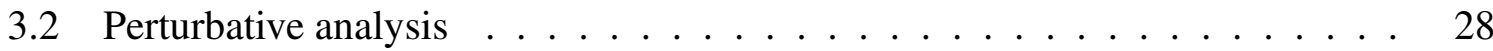

3.2.1 Periodic potentials . . . . . . . . . . . . . . . 28

3.2.1.1 Non-degenerate energy levels . . . . . . . . . . . . . . 29

3.2.1.2 Energy levels near the Bragg plain . . . . . . . . . . . . . 30

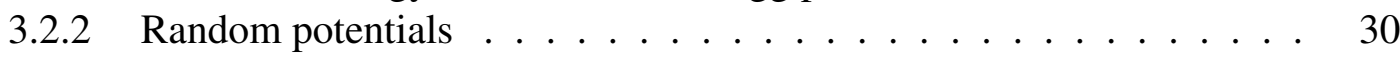

3.3 Dynamics on intermediate scales . . . . . . . . . . . . . . 31

3.3.1 Periodic potentials . . . . . . . . . . . . . . 31

3.3.1.1 Ordinary diffusion . . . . . . . . . . . . 32

3.3.1.1.1 Cosine potential . . . . . . . . . . 32

3.3.1.1.2 Piecewise potential ................ 33

3.3.1.1.3 Peaks and wells potentials ......... . . 34

3.3.1.2 Superdiffusion . . . . . . . . . . . . . . 36

3.3.1.2.1 Cosine potential . . . . . . . . . . . 37

3.3.1.2.2 Piecewise constant potential . . . . . . . 38

3.3.1.2.2.1 Kronig-Penney method . . . . . . . 39

3.3.1.2.3 Wells and Peaks potentials . . . . . . . . . 40

3.3.2 Random potentials . . . . . . . . . . . . . . . . 42

3.3.2.1 Ordinary diffusion . . . . . . . . . . . . . . 43

3.3.2.2 Superdiffusion . . . . . . . . . . . . . . 44

3.4 Behavior on large scales $\ldots \ldots \ldots \ldots$. . . . . . . . . . . . 46 
3.4.1 Periodic potentials ....................... 46

3.4 .2 Random potentials . . . . . . . . . . . . . . . . 46

3.5 Numerical results . . . . . . . . . . . . . . . . . . . . . . . . . 48

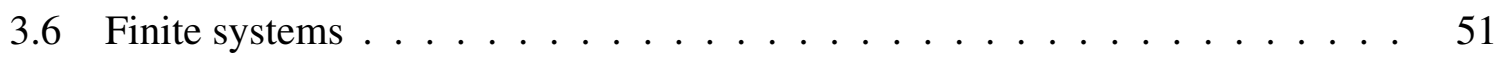

3.7 Global coupling . . . . . . . . . . . . . . . . . 53

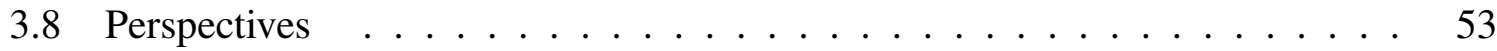

3.9 Summary and discussions $\ldots \ldots \ldots \ldots \ldots$

4 Epidemic spread due to recurrent host movements $\mathbf{5 8}$

4.1 General model . . . . . . . . . . . . . . . . . . . 58

4.1.1 Recurrent movements on star-like topologies . . . . . . . . . . . 60

4.1.1.1 Stationary properties . . . . . . . . . . . . 61

4.1 .2 Interacting species $\ldots \ldots \ldots 63$

4.2 Direct versus bidirectional coupling . . . . . . . . . . . . . . 64

4.3 Exemplary case of two populations . . . . . . . . . . . . . . . . 66

4.4 Epidemics on continuum support . . . . . . . . . . . . . . 68

4.4.1 Epidemics due to recurrent transport . . . . . . . . . . . . . 68

4.4 .2 Front velocity . . . . . . . . . . . . . . . . 71

4.4.3 Dependence of the front velocity on the travel rate . . . . . . . 73

4.4.4 Dependence on the commuter ratio . . . . . . . . . . . . . . . . . 74

4.4.5 Dependence on the infection rate . . . . . . . . . . . . 76

4.4.6 Front shape . . . . . . . . . . . . . . . . . . 77

4.4.7 Epidemics with direct coupling . . . . . . . . . . . . . . . . . . . 79

4.4.7.1 Front velocity . . . . . . . . . . . . . 82

4.4.7.2 Front shape .................. . . 83

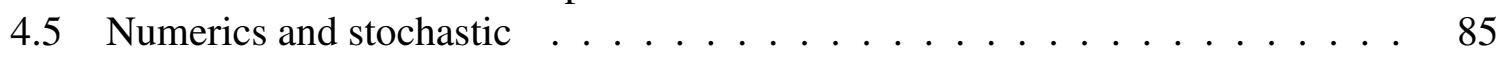

4.5.1 Brunet-Derrida scaling . . . . . . . . . . . . . . 86

4.5.2 Behavior of the front velocity for small travel rates . . . . . . . . . . . 87

4.6 Epidemics on networks . . . . . . . . . . . . . . . . . . 90

4.6.1 Modelling travel rates . . . . . . . . . . . . . . . . 91

4.6.1.1 Topological disorder . . . . . . . . . . . . . 93

4.6.1.2 Equal commuter ratios independent of the location . . . . . 94

4.6.1.3 Non-equal commuter ratios without normalization . . . . . 95

4.6.1.4 Rates dependent on the actual population sizes . . . . . . . 96

4.6.1.5 Available flows . . . . . . . . . . . . . . 96

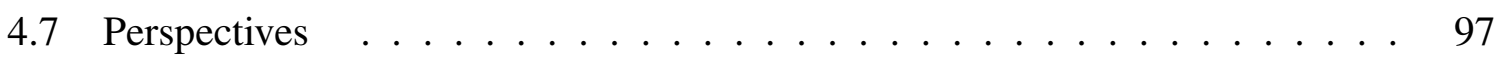

4.8 Summary and discussion . . . . . . . . . . . . . . . 100

5 Conclusion $\quad 103$

$\begin{array}{ll}\text { A Bloch theory } & 107\end{array}$

B Perturbation theory for random potentials 109 
Acknowledgments

113

Bibliography 


\section{Introduction}

In recent decades, there have been many successful attempts to describe various phenomena emerging in human society and animal systems deploying approaches, proven to be useful in natural sciences, especially in physics. To name a few of them: epidemics (Anderson and May, 1991), prey-predator ecological interactions (Holland and Hastings, 2008), traffic jams (Helbing, 1997) and panic reactions (Helbing et al., 2000). In these approaches, individuals are considered as abstract interacting agents endowed with properties essential to account for the behavior of the whole system. In the past the modeling of multi-agent systems was limited by the lack of reliable real data. Recent progress in this field can be primarily attributed to developments in communication technologies. Powerfull computer facilities and ubiquitous GPS devices have made it possible the collection, processing and storage of the data on individual behavior (González et al., 2008; Cooke et al., 2004).

The collected data reveals the complexity of phenomena emerging in multi-agent systems. Thus, the need for guiding principles arises which facilitates the understanding of dynamics, allows assessment of crucial factors and their incorporation into models. Basic, easily tractable mathematical models may provide indispensable insights into the behavior of complex systems. In this thesis we will consider two such models. The first deals with superdiffusion in inhomogeneous environments. The second describes epidemics mediated by recurrent movements of agents.

Epidemiology was the main field of application that we had in mind during preparation of the thesis. Epidemics have been accompanying mankind throughout history, with serious social and cultural consequences (Winkle, 2005). For example, during the Bubonic plague epidemic in Middle Ages, 30-40 percent of the total European population was eliminated. Since the early eighties, AIDS have caused 25 million deaths worldwide. Though mathematical epidemiology may not be the paramount factor in the battle against epidemics - which belongs to the advances of molecular biology and genetic engineering - it may help to save a lot of resources by helping to plan preventive and respond measures (Anderson and May, 1991).

Infectious diseases are carried by agents - either animals or humans - called hosts. There are two aspects of the spatial spread of infectious diseases (Riley, 2007). The first one concerns host contacts within a population; the other involves contacts between hosts from different populations. For a long time, interaction between different populations was accounted for by the assumption of the diffusive movements of the hosts. For example, the historic epidemic of Bubonic plague was modeled in this way (Noble, 1974). In this type of models, the local infection dynamics (reaction) is decoupled from the transport (diffusion) (Kolmogorov et al., 1937; Fisher, 1937). Due to the highly developed means of transportation, people nowadays are able to cover much greater distances within shorter timeframe as compared to the past (Cliff and Haggett, 2004). Contrary to ordinary diffusion (Gardiner, 1997)the human- 
mediated spread exhibits superdiffusive properties (Brockmann et al., 2006) closely related to power-law distributions (Metzler and Klafter, 2000, 2004). Some animals were also found to perform superdiffusive random walks (Sims et al., 2008).

On large scales, humans move between different locations, e.g. cities or villages, which differ in population size. Contact patterns among individuals and their travel behavior possess scale-free and small-world properties (Liljeros et al., 2001; González et al., 2008) witnessing strong social inhomogeneity. Transportation routes represent a random network rather than a regular lattice (Gastner and Newman, 2006). Therefore, a concept of inhomogeneity or disorder is indispensable for appropriate models of human-mediated transport.

The main question pursued in the first part of this thesis is how inhomogeneity affects spreading phenomena, especially if long-range interactions are allowed. Incorporation of the inhomogeneity into the theory of superdiffusive processes is a non-trivial task. Depending on the system under consideration different approaches are deployed. In contrast to the generalized Langevin approach (Fogedby, 1998; Metzler and Klafter, 2000) the topological superdiffusion (Brockmann and Sokolov, 2002; Brockmann and Geisel, 2003a,b; Chen and Deem, 2003), reveals that weak inhomogeneity is present on all scales. Following this approach, we generalize the topological superdiffusion concept, explicitly taking into account the relative weight of start and target location of a single movement event. Our model is reminiscent of the gravity model widely used in epidemiology, social sciences and economics (Xia et al., 2004; Zipf, 1946; Wilson, 1967). The gravity model states that the flow of goods or individuals between two different locations is directly proportional to the population sizes of these locations, and inversely proportional to some power of the distance between them.

Using our model we show, that if agents perform ordinary diffusion, the spreading process is attenuated by inhomogeneity on large scales. In contrast, if superdiffusive movements are allowed the spread can be also accelerated on large scales, depending on the relative impact of source and target positions (Belik and Brockmann, 2007). It is a counterintuitive result because usually inhomogeneity attenuate diffusion. This result holds for periodic as well as random inhomogeneities.

Power-law distributions of jump lengths are often considered as suitable candidates for an optimal strategy in random search problems (Condamin et al., 2008). The intermediate values of the power law exponent are supposed to enhance exploration by a random walker and thus represent optimal search strategy (Viswanathan et al., 1999b). However, accounting for finite size effects within our model, we show that in the presence of inhomogeneity the whole range of power law exponents - from diffusive to superdiffusive — can be optimal. The particular choice depends on the impact of temporary source and target locations.

To adequately describe spatial epidemic dynamics, we consider patterns of human movements in detail. Humans spend the most of their time at home and in the workplace. From time to time, they leave their homeplace to perform trips to neighboring locations, or distant places and then return. Thus on large scales they do not perform random walks, hopping among all possible locations; they rather perform recurrent movements or commute (González et al., 2008). The reaction-diffusion approach that assumes unbounded agent movements can not be applied in this case. To account for the effective coupling of the epidemic outbreaks between different locations, a heuristic approach was proposed, in which recurrent travel mechanism is not considered explicitly. The model states that the rate of change of the number of infectives 


\section{Introduction}

in one location is proportional to the number of infectives in other locations with the factors of proportionality called coupling strengths (Rushton and Mautner, 1955). This model referred to as the direct coupling model is widely used in epidemiological modeling (Grenfell et al., 2001). Surprisingly enough, little effort was devoted to explicit incorporation of the recurrent travel behavior into a microscopic epidemiological model. The goal of the second part of our work is to fill this gap.

The main question pursued in the second part of our work concerns the role of the recurrent transport mechanism in the spread of infectious diseases. Starting from the microscopic domain we build up the general model for host movements with travel rates dependent on the location of origin. In a special case our model describes recurrent travel pattern on overlapping star-like topologies (Sattenspiel and Dietz, 1995). We go beyond the formulation of general ideas and build a continuum version of the model describing epidemic due to recurrent travel on a one-dimensional lattice of locations. We analyze it in dependence on parameters such as travel and infection rates and get essential insight into the dynamics of epidemics due to recurrent hosts movements. We obtain attenuation of the epidemic wave as compared to the common reaction-diffusion model as the major consequence of the recurrent travel. We show that with increasing travel rate, the velocity of the epidemic wave in the recurrent case saturates towards its asymptotic value. It is in contrast to the ordinary diffusion-reaction approach leading to unbounded growth of the velocity with the travel rate.

We show that model with recurrent travel can in general not be reduced to neither the directly coupled metapopulational epidemic model nor to the reaction-diffusion model. We demonstrate that the directly coupled model recovers only in the limit of high travel rates. We explicitly calculate the elusive coupling strengths of the direct coupled model from the host travel rates. With respect to the last mentioned result, some of our findings were independently anticipated by Keeling and Rohani (2005), although we proceed more systematically. Detailed numerical investigations of our model implies similarities between the reaction-diffusion case and the recurrent movement case for a high ratio of reaction rate versus travel rate.

Regular topologies constitute a crude approximation of real settings more reminiscent of random networks. Epidemics on networks were extensively explored in the last decades. The most studies were dedicated to networks with nodes representing single hosts and edges mimicking social ties (Newman, 2002). Different nodes of the network can also represent different populations (locations) with links corresponding to transportation routes between them (Hufnagel et al., 2004; Collizza et al., 2007). We discuss this type of network models. In the spirit of the first part of the thesis we also consider inhomogeneously populated locations and propose several plausible models for parameters of recurrent travel, e.g. travel rates.

In this thesis we reveal the important role of inhomogeneity in systems with long-range movements and shed light on the consequences of recurrent host movements for spatio-temporal dynamics of epidemics. We are convinced that our work contributes to the thorough theoretical understanding of various spreading phenomena in the modern world.

In the course of our work we have used various analytical and numerical methods. In the first part that concern superdiffusion in inhomogeneous environments, we have used Bloch band theory for periodic media as well as the perturbational techniques to calculate spectral properties of the transport operator. We have also used numerical diagonalization to support our analytical results. In the second part that concerns epidemics mediated by recurrent 


\section{Introduction}

movements we have used linear stability analysis, numerical integration and full stochastic agent-based simulations.

The work is structured as follows. In Chapter 2, we introduce basic concepts necessary for understanding the following material, including superdiffusion, gravity model, and basic epidemiological concepts. In Chapter 3, we study superdiffusion in inhomogeneous periodic and random correlated environments. We define the model, then investigate it on intermediate and large scales. In Chapter 4, we consider epidemic spread due to origin-dependent travel rates and formulate a general model. After that we derive a continuum model for a onedimensional support. Then we relate the general model as well as its continuum version to the direct coupled model and investigate it numerically in a wide range of parameters. We also provide comparison with the reaction-diffusion framework. Eventually, we discuss modeling approaches to epidemics on networks. In Chapter 5, we discuss the main results and draw conclusions. Details concerning Bloch theory and the derivation of perturbational results can be found in the Appendix. 


\section{Fundamentals}

In the current chapter we will present concepts indispensable for the understanding of the results presented in the following chapters. We start with the notion of superdiffusive processes, proceed with the concept of disorder, mention different ways to incorporate it, in particular an approach inspired by gravity models, recall common epidemiological models and eventually introduce the reaction-diffusion framework.

\subsection{Superdiffusion}

Before we proceed to superdiffusion, we need to recall the basics of ordinary diffusion and the underlying concept of random walk. Experimental observation of diffusion is due to Robert Brown (Brown, 1866), who in 1927 investigated pollen suspension in water and observed their perpetual movements. Such movements bear his name. They are called Brownian motion and represent an example of diffusion. Note, that he had a predecessor - a Dutch physician Jan Ingenhousz observed a similar phenomenon around 1785. The pollen move because of collisions with molecules of the water which always perform thermal movements. Adolf Fick (Fick, 1855) has derived the diffusion equation for concentration of some fluid substance using purely phenomenological approach without considerations on microscopic level. For a long time the honor of explanation and theoretical description of the Brownian motion, and thus diffusion, was ascribed to Albert Einstein (Einstein, 1905). However ordinary diffusion was theoretically considered already in 1900 by Louis Bachelier in his for a long time erroneously forgotten study (Bachelier, 1900) in the context of fluctuations of stock market prices. He considered prices as a random walk, i.e. jumps occurring in different directions according to some probability. Bachelier also derived the diffusion equation and solved it successfully. But even before that earlier Lord John Reighley derived the diffusion equation from essentially the random walk ansatz. Among the early contributors to the subject one can also mention Thorwald Nicolai Thiele and Marian Smoluchowski. One can find more details on the early history of the study of diffusion phenomena in (Montroll and Shlesinger, 1984; Ebeling et al., 2008).

A particle performing ordinary diffusion is typically characterized by spatio-temporal scaling relation $|X(t)| \sim t^{1 / 2}$, where $|X(t)|$ denotes a displacement from the starting point. A large number of physical and biological systems are in conflict with this relation and exhibit anomalous diffusion. Whenever $|X(t)| \sim t^{1 / \mu}$ with an exponent $0<\mu<2$ a system is said to exhibit superdiffusive behavior. In Figure 2.1, random walk underlying ordinary diffusion is compared to superdiffusive movements or Lévy flights named after the French mathematician Paul Lévy. He elaborated the mathematical foundations of the theory of Lévy flights (Lévy, 1954). Superdiffusion was discovered in a wide range of systems, for instance, chaotic dy- 
(a)

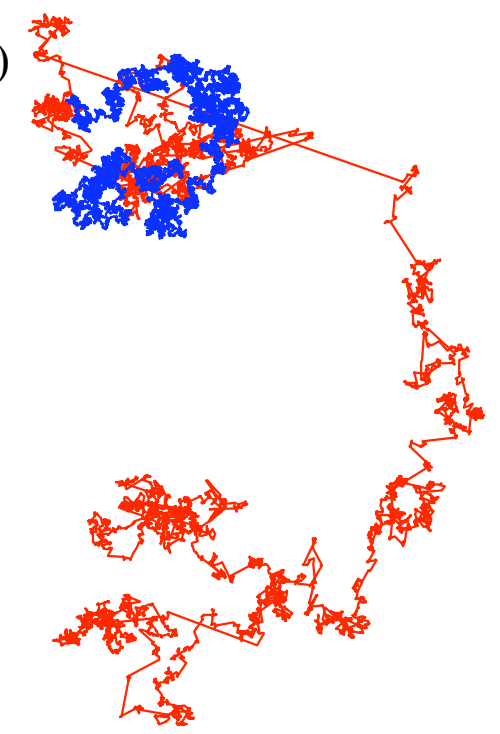

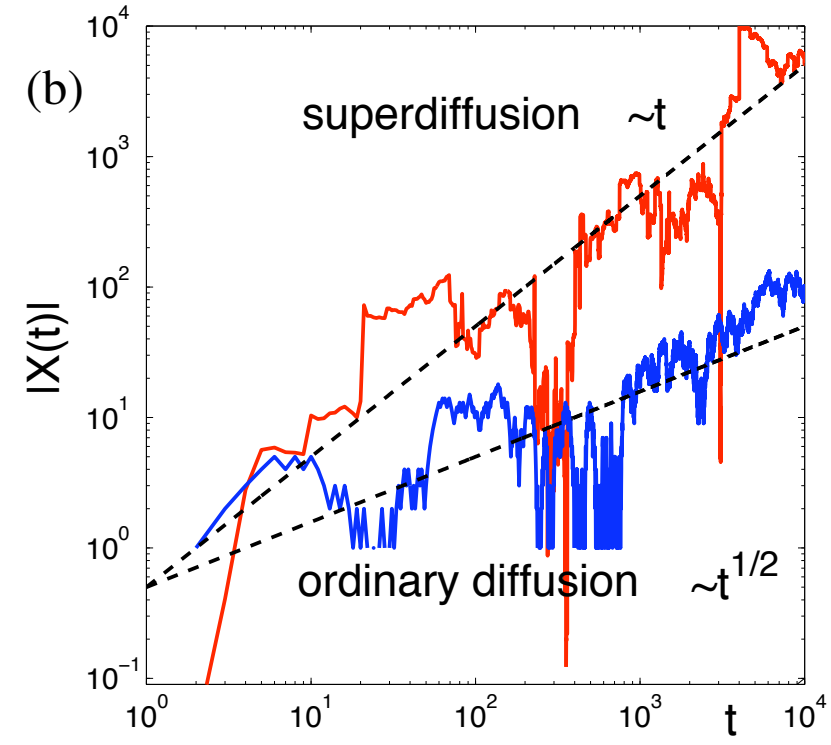

Figure 2.1: Panel (a): ordinary random walk (blue) characterized by localized movements and superdiffusive Lévy flights lacking a characteristic scale (red). In superdiffusive case long jumps are interspersed with clusters of local movements. Panel (b): scaling properties of ordinary random walk and superdiffusive Lévy flights. The dependence of displacement $|X|$ on time $t$ is presented for ordinary diffusion and superdiffusion $(\mu=1)$. Dashed lines represent scaling asymptotics.

namical systems (Geisel, 1985), particles in turbulent flows (Porta et al., 2001), saccadic eye movements (Brockmann and Geisel, 2000), and very recently in the geographic dispersal of bank notes (Brockmann et al., 2006).

After these historical remarks we proceed now to a theoretical description of diffusion. Let us consider a Markov stochastic process, i.e. a set of states of a system at different times with random increments $x\left(t_{0}\right), x\left(t_{1}\right), \ldots, x(t)$ which can be interpreted as a position in state or real space obeying the Chapman-Kolmogorov equation (Gardiner, 1997; van Kampen, 2007; Ebeling and Sokolov, 2006; Honerkamp, 1990)

$$
p\left(x, t \mid x_{0}, t_{0}\right)=\int d y p\left(x, t \mid y, t^{\prime}\right) p\left(y, t^{\prime} \mid x_{0}, t_{0}\right) .
$$

Here $p\left(x, t \mid x_{0}, t_{0}\right)$ denotes the probability to be at some particular position $x$ at time $t$ having started at time $t_{0}$ at $x_{0}$ depends only on the position $y$ at the previous time $t^{\prime}$ and the transition probability from that position into the current location. Let us choose a Markov process with transition probabilities for small time intervals $\tau$ given by

$$
p(x, t+\tau \mid y, t)=[1-a(y ; t) \tau] \delta(y-x)+\tau w(x \mid y ; t)+\mathcal{O}\left(\tau^{2}\right)
$$

with

$$
a(y, t)=\int d x w(x \mid y ; t) .
$$


This condition means that the process will keep its current location $x$ with a probability $1-a(y, t) \tau$ or will jump to a new location $y$ with the probability $w(x \mid y ; t) \tau$. The quantity $w(x \mid y ; t)$ is called the transition or transfer rate. We will not consider its time dependence: $w(x \mid y ; t)=w(x \mid y)$. From (2.1)-(2.3), under consideration of $p(x, t)=\int d y p(x, t \mid y, t)$, and performing the limit $\tau \rightarrow 0$ we obtain the Master equation

$$
\partial_{t} p(x, t)=\int d y[p(y, t) w(x \mid y)-p(x, t) w(y \mid x)]
$$

If we consider an ensemble of random walkers we can describe their distribution in space at any time by the probability density function $p(x, t)$ fulfilling the Master equation, which is essentially the balance equation. This means that the rate of change of the probability density at point $x$ consists of the contributions due to walkers coming to $x$ from all possible $y$ minus the contribution due to walkers going away from $x$ to any possible $y$.

The Master equation can be further simplified. If we assume the transition rates being sharply peaked as a function of the jump size, the equation known as the Fokker-Planck equation among physicists and as the Kolmogorov forward equation among mathematicians, is recovered. It can be written as

$$
\frac{\partial}{\partial t} p(x, t)=-\frac{\partial}{\partial x}[a(x) p(x, t)]+\frac{\partial^{2}}{\partial x^{2}}[b(x) p(x, t)]
$$

with

$$
a(x)=\int d r r w(x ; r) \text { and } b(x)=\int d r r^{2} w(x ; r)
$$

being the conditional moments. Here and in the following, if is stated explicitely, we integrate over the whole infinite domain. The transition probability is considered here to be a function of the starting point $y$ and the jump length ${ }^{1} r=x-y$. The first term is called the drift term and the second term is called the dispersive or diffusive term. Note that the Master equation is of a more general nature than the Fokker-Planck equation. The latter can be obtained from the Master equation under some restrictions on transition rates.

Note, that in the case when transition rates are symmetric, the drift term vanishes. For instance, if we assume that $w(x \mid y)=\delta(x-y-a)+\delta(x-y+a) /(2 \tau)$ for $|x-y|<a$ with

${ }^{1}$ Indeed if we define $w(x \mid y)=w(y ; r)$ then from $(2.4)$

$$
\partial_{t} p(x, t)=\int d r w(x-r ; r) p(x-r ; t)-p(x, t) \int d r w(x ;-r)
$$

and assuming slow variation of $p(x, t)$ as a function of $x$ using the Taylor expansion up to the second order (the full Taylor expansion in this case is called Kramer's-Moyal expansion) we obtain

$$
\begin{aligned}
\partial_{t} p(x, t)=\int d r w(x ; r) p(x ; t)-\int d r r & {\left[\frac{\partial}{\partial x} w(x ; r) p(x, t)\right] } \\
& +\frac{1}{2} \int d r r^{2}\left[\frac{\partial}{\partial x} w(x ; r) p(x, t)\right]-p(x, t) \int d r w(x ;-r) .
\end{aligned}
$$

From the last equation follows the Fokker-Planck equation (2.5). There are also other methods to derive the Master equation, for example the system size expansion by van Kampen (van Kampen, 2007). 
characteristic jump length $a>0$ and time scale given by $\tau$, then we obtain from the ordinary diffusion equation (2.5)

$$
\partial_{t} p(x, t)=D \Delta p(x, t)
$$

where the constant $D=a^{2} / \tau$ is called the diffusion coefficient. Note that for Equation (2.5) to be correct we need to consider our process on scales larger than $a$ to obtain a continuous description. The characteristic time increment $\tau$ also needs to be small enough but such that $D$ is kept constant. The solution of the equation (2.7) is given by

$$
p(x, t)=\frac{1}{\sqrt{2 \pi D t}} \exp \left[-\frac{x^{2}}{(2 D t)^{2}}\right],
$$

i.e. it is a Gaussian. From this, one obtains the scaling of the absolute value of the coordinate $|x(t)| \sim t^{1 / 2}$ depicted in Figure 2.1(b). Thus (2.7) describes statistical properties of the random walk with continuous time.

If we allow for long range jumps by introducing the transition rate

$$
w(x \mid y)=\frac{a^{\mu} \mu}{2 \tau} \begin{cases}0 & |x-y|<a \\ |x-y|^{-1-\mu} & |x-y|>a\end{cases}
$$

with $0<\mu<2$, we cannot write down the usual Fokker-Planck equation because the second moment of the transfer rate is diverging. Instead, from the Master equation (2.4) we obtain

$$
\partial_{t} p(x, t)=\frac{a^{\mu} \mu}{2 \tau} \int_{|x-y|>a} d y \frac{p(y, t)-p(x, t)}{|x-y|^{1+\mu}},
$$

which can be rewritten in the limit $a \rightarrow 0$ and $\tau \rightarrow 0$ as

$$
\partial_{t} p(x, t)=D_{\mu} \Delta^{\mu / 2} p(x, t),
$$

where we have kept the superdiffusion coefficient $D_{\mu}=\left(a^{\mu} / \tau\right) \pi \mu /[\Gamma(1+\mu) \sin (\pi \mu / 2)]$ constant and introduced the fractional Laplacian $\Delta^{\mu / 2} f(x)=\mathcal{C}_{\mu} \times$ p.v. $\int d y \frac{f(y)-f(x)}{|x-y|^{1+\mu}}$ with $\mathcal{C}_{\mu}=\Gamma(1+\mu) \sin (\pi \mu / 2) / \pi$. In Fourier space, it is equivalent to multiplication with $-|k|^{\mu}$ (Hilfer, 2000). Solving (2.9) in the Fourier space we obtain

$$
p(x, t)=\frac{1}{2 \pi} \int d k e^{-k x-D|k|^{\mu} t} .
$$

Hence, we find the scaling $\left|x_{\mu}(t)\right| \sim t^{1 / \mu}$, which is also depicted in Figure ??. Random walks obeying condition (2.8) are called Lévy flights. Such processes possess self-similar fractal properties (See Figure 2.1).

Another way to describe stochastic processes is to use stochastic differential equations. One can show (Gardiner, 1997) that the Fokker-Planck equation (2.5) is equivalent to the stochastic differential equation for the increment of the stochastic process $X(t)$

$$
d X=a(X, t)+\sqrt{b(X, t)} d W
$$




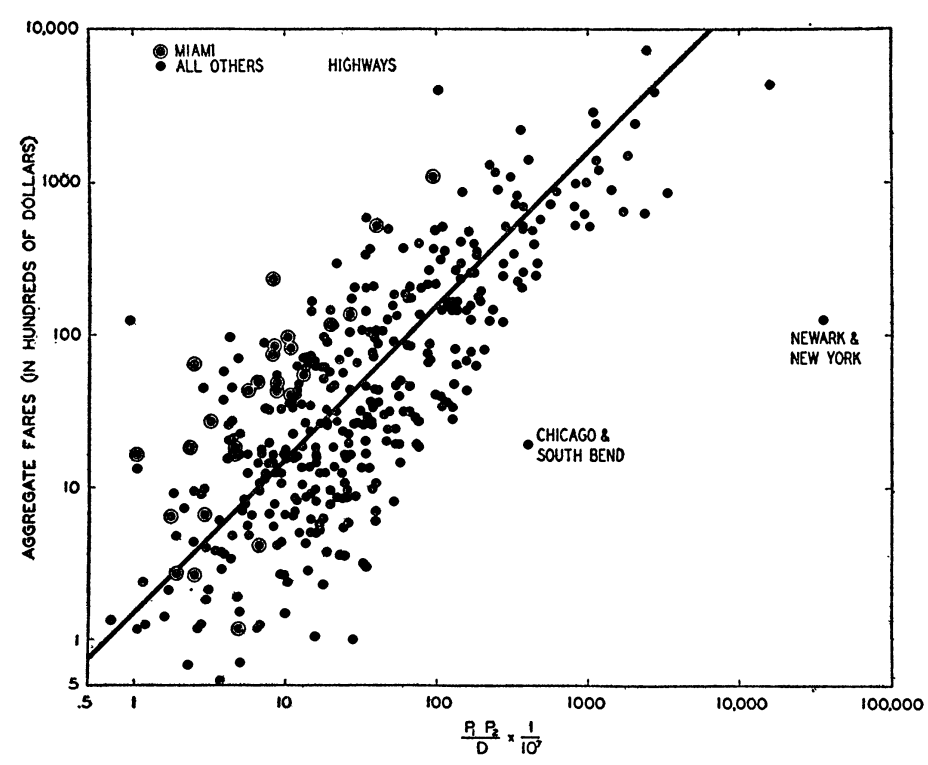

Figure 2.2: Empirical evidence for the gravity model (Zipf, 1946). Dependence of the aggregate fares paid by the highway bus passengers on the quantity $P_{1} P_{2} / r \times 10^{-7}$. Here $P_{1}$ and $P_{2}$ denote sizes of a pair of locations and $r$ denotes distance between them. Solid line has the slope of -1 , supporting the gravity model hypothesis.

where $d X$ and $d W$ are limits of the differences $\Delta X=X(t+\Delta t)-X(t)$ and $\Delta W=$ $W(t+\Delta t)-W(t)$ for $\Delta t \rightarrow 0 . \Delta W$ represents a difference of a Wiener process and is a Gaussian random variable with zero mean and variance of $\Delta t$. For the definition of the stochastic differential $d W$, one can take either the Ito or the Stratonovich interpretation (Gardiner, 1997; Stratonovich, 1963). One can also rewrite the stochastic differential equation as the Langevin equation

$$
\frac{d x}{d t}=a(x, t)+\sqrt{b(x, t)} \xi(t)
$$

where $\xi(t)$ is a stochastic force with properties $\langle\xi(t)\rangle=0$ and $\left\langle\xi(t) \xi\left(t^{\prime}\right)\right\rangle=\delta\left(t-t^{\prime}\right)$. It is called white noise. Note, that the Langevin equation possesses some cumbersome mathematical properties. $\xi(t)$ with infinite variance corresponds to no real process. This requires consideration of $\xi(t)$ as a limiting case of a properly defined process which turns to be very complicated (Gardiner, 1997). If we discard fluctuations in Equation (2.11), we deduce the equation describing macroscopic, mean-field dynamics

$$
\frac{d x}{d t}=a(x, t)
$$

Let us recall that the conditional moment $a(x, t)$ could be derived from the corresponding transition probability $w(x \mid y ; t)$ by using definition (2.6).

\subsection{Disorder and gravity model}

A superdiffusive process governed by Equation (2.9) is spatially homogeneous and isotropic, as the probability rate $w(y \mid x)$ of a displacement from $x$ to $y$ depends only on the distance, e.g. for the $d$-dimensional case $w(\boldsymbol{x} \mid \boldsymbol{y}) \propto|\boldsymbol{x}-\boldsymbol{y}|^{-(d+\mu)}$. Numerous random processes, however, occur on spatially disordered substrates or evolve in the presence of quenched or 
frozen spatial inhomogeneities. There exist a variety of phenomena requiring disorder which are completely absence without it. Geometrical type of disorder leads to percolation phase transitions (Hughes, 1995b). This type of disorder arises in problems of diffusion on fractal structures (ben Avraham and Havlin, 2000) or random networks (Kozma et al., 2005) We discuss such kinds of disorder in Chapter 4 in Section 4.6. Our main concern will be inhomogeneities which can be modeled by position dependent transition rates of a random walk (Bouchaud and Georges, 1990; Haus and Kehr, 1987). For example the charge transport in the presence of impurities in solid state physics leading to localization (Anderson, 1958), could be described in such a framework.

Using the Fokker-Planck equation, disorder can be incorporated into the model of ordinary diffusion in a straightforward way. Let us restrict ourselves to systems with time-independent parameters. One can show that the first conditional moment $a(x)$ from Equation (2.5) equals the external force $F(x)$ with a minus sign. The second conditional moment $b(x)$ may be interpreted as a position-dependent diffusion coefficient $D(x)$. Both the external force and the position-dependent diffusion coefficient can account for disordered environment (van Kampen, 2007) $)^{2}$

$$
\frac{\partial p(x, t)}{\partial t}=-\frac{\partial F(x) p(x, t)}{\partial x}+\frac{\partial^{2} D(x) p(x, t)}{\partial x^{2}} .
$$

In the case of superdiffusive movements, the situation is more involved. Depending on the underlying physical or biological system, one obtains various generalizations of (2.13) which incorporates the spatial inhomogeneities. In the generalized Langevin approach (Fogedby, 1998; Metzler and Klafter, 2000, 2004), an additional force term $-\nabla F$ on the right hand side of Equation (2.9) accounts for an external position dependent force field $F(x)$. In topologically superdiffusive systems, such as intersegment transfer of gene regulatory enzymes on DNA strands (Brockmann and Geisel, 2003b; Sokolov et al., 2005; Lomholt et al., 2005; Chen and Deem, 2003), the transition rate is modified by a Boltzmann factor, i.e. $w(y \mid x) \propto$ $|x-y|^{-(d+\mu)} \times \exp \{-\beta[V(y)-V(x)] / 2\}$, where $V(x)$ is a position-dependent potential and $\beta$ denotes inverse temperature. In subordinated superdiffusive processes, an ordinary diffusion process subject to an external force is sampled at highly variable operational time intervals (Sokolov, 2000). All three systems exhibit very different response properties to the imposed spatial structure (Brockmann and Sokolov, 2002), but converge to the same FokkerPlanck equation in the limit of ordinary diffusion.

Inhomogeneities, in particular in the population structure can also be encountered very frequently also in ecological and epidemiological problems. For a long time the flows between inhomogeneously populated locations were approximated by the so-called gravity models. The early history of the gravity model concept dating into XIX-th century is described by Garrothers (Garrothers, 1956). Empirical evidence was provided in the forties of the XX-th century (Zipf, 1946). For recent studies see e.g. (Jung et al., 2008; Viboud et al., 2006; Xia et al., 2004).

The gravity model assumes that flows of individuals or goods between two locations $n$ and $m$ are proportional to the population sizes of both of them $F_{n m}=F_{m n} \sim P_{n} P_{m}$. Further

\footnotetext{
${ }^{2}$ Dependent on the system under consideration, the proper Fokker-Planck equation for inhomogeneous environment reads $\partial_{t} p=-\partial_{x}\left[F(x) p+D(x) \partial_{x} p\right]$ (van Kampen, 2007; Bouchaud and Georges, 1990).
} 
one can introduce another refinement of the model concerning the dependence on the distance between locations. In Figure 2.2 the dependence of aggregate fares paid by bus highway passengers reflecting the flow of individuals on the quantity $P_{1} P_{2} / r \times 10^{-7}$ is presented. One can observe a good agreement a proposed gravity '. Originally the interlocation flow was supposed to be inversely proportional to the distance, but it can be modified to be $F_{n m} \sim r_{n m}^{-2-\mu}$ with $0<\mu<2$ mimicking the superdiffusive nature of human mediated spread (Brockmann et al., 2006). One can show that the gravity model comes out as a solution of the entropy optimization problem (Wilson, 1967; Erlander, 1980). Thus we can set up a model incorporating the gravity model approach and superdiffusion. For the transition rate in the continuum case we can write

$$
w(x \mid y) \sim \frac{P(x)^{c} P(y)^{c-1}}{|x-y|^{d+\mu}}
$$

with $P(x)$ giving us the population density on point $x$. Population sizes in this formula are often additionally raised to power $c$, what is a common practise (Xia et al., 2004). Inserting this transition rate into the Master equation (2.4) the following fractional Fokker-Planck equation can be derived

$$
\partial_{t} p=D s^{c} \Delta^{\mu / 2} s^{(c-1)} p-D p s^{c-1} \Delta^{\mu / 2} s^{c},
$$

where instead of the population densities the salience field $s(x)$ accounting for attractiveness is introduced. High salience corresponds to the higher attractiveness of a location ${ }^{3}$. It may be assumed to be proportional to the population density $s(x) \sim P(x)$. Chapter 3 is devoted to the investigation of the equation (2.14) in different inhomogeneous environments, represented by different models for the inhomogeneous salience field.

\subsection{Basic epidemiological models}

In order to understand the spatio-temporal models of the spread of infectious diseases considered in Chapter 4, we have to recall the basic mathematical framework used in epidemiology. For the description of local infectious dynamics in an isolated population, the compartmental models have proven their usefulness (Anderson and May, 1991). In such kinds of models the population is divided into classes or species according to their disease status, e.g. susceptible or healthy individuals, infected and latent individuals. Different species can interact with each other producing new species and generally can have its own birth and death dynamics. Furthermore, one usually a well-mixed population, i.e. everybody can contact everybody and the frequency of contacts is proportional to the densities of species. Thus such a system can be described by the standard chemical kinetics rules (van Kampen, 2007). Individuals are described as particles experiencing collisions in volume $\Omega$ at constant temperature. If we have chemical reactions among different $M$ species described by the equation

$$
s_{1} X_{1}+s_{2} X_{2}+\ldots \stackrel{k_{+}}{\rightarrow} r_{1} X_{1}+r_{2} X_{2}+\ldots
$$

\footnotetext{
${ }^{3}$ The term salience means something most noticeable or important and comes from the neuroscience of visual field processing, see e.g. (Brockmann and Geisel, 2000).
} 
then the rate of transition from the state $\left\{n_{j}\right\}$ describing the number of species $j$ into the state $\left\{n_{j}+r_{j}-s_{j}\right\}$ due to reaction (2.15) reads

$$
w\left(n_{j}+r_{j}-s_{j} ; n_{j}\right)=\Omega k_{+}\left(r_{i}-s_{i}\right) \prod_{j=1}^{M}\left(\frac{n_{j}}{\Omega}\right)^{s_{j}} .
$$

Such a description is called the frequency dependent model. Without a loss of generality, we can set the density $\rho=N / \Omega$ to unity. Using (2.6) and (2.12) one can write down mean-field equations for the average number of species, e.g.

$$
\frac{d n_{j}}{d t}=N k_{+}\left(r_{i}-s_{i}\right) \prod_{j=1}^{J}\left(\frac{n_{j}}{N}\right)^{s_{j}}
$$

Now we discuss three frequently used epidemiological models based on the approach of chemical kinetics.

\subsubsection{SI model}

The most simple reaction scheme includes susceptibles and infectives, which can infect susceptibles. Infectives keep their infective status forever. This model is appropriate for description of chronic infectious diseases with no immunity. Birth and death dynamics are neglected in this model which correspond to consideration of the epidemic on time scales smaller than a life period of the host. The reaction scheme reads

$$
S+I \stackrel{\alpha}{\rightarrow} 2 I
$$

with corresponding mean-field equation

$$
\frac{d I}{d t}=\frac{\alpha}{N}(N-I) I
$$

where we have used conservation of the individuals $N=S+I$. In terms of the concentrations $j=I / N$

$$
\frac{d j}{d t}=\alpha(1-j) j
$$

For a given initial condition $j(0)=j_{0}$ the solution reads

$$
j(t)=\frac{j_{0}}{\left(1-j_{0}\right) e^{-\alpha t}+j_{0}} .
$$

The evolution of the number of infectives and susceptibles as well as their stochastic counterparts are presented in Figure 2.3(a). Zero number of infectives is an unstable fixed point and $j^{\star}=1$ is a stable one. A non-zero stable fixed point is called the endemic steady state. 

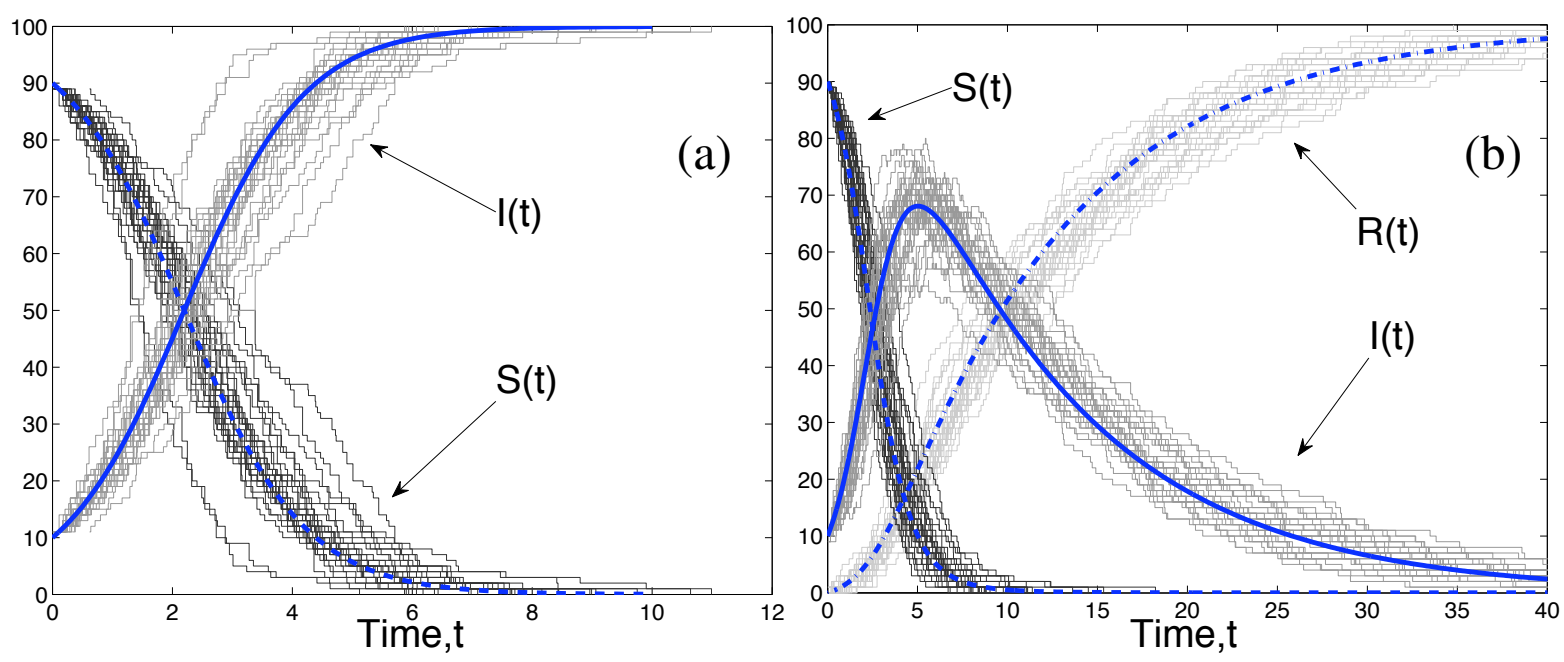

Figure 2.3: Time evolution of SI (a) and SIR (b) epidemics. Blue lines denote solutions of the mean field equations (2.17) and (2.18). Grey lines correspond to results of stochastic simulations of 30 different realisations. Population size $\mathrm{N}=100$, infection rate $\alpha=1$, recovery rate $\beta=0.1$ and $I(0)=$ 10.

\subsubsection{SIR model}

This SIR model was initially proposed by Kermack and McKendrick and it distinguishes between three classes of individuals: susceptibles, infectives and recovered (immune) or removed (Kermack and McKendrick, 1927). Along with infection of susceptibles, the infectives can recover or die. This model could be appropriate for either very dangerous diseases with high mortality or diseases leading to strong immunity against them. The reaction scheme reads

$$
\begin{aligned}
& I+S \stackrel{\alpha}{\rightarrow} 2 I \\
& I \stackrel{\beta}{\rightarrow} R
\end{aligned}
$$

The mean field equations look like

$$
\begin{aligned}
& \frac{d j}{d t}=\alpha s j-\beta j \\
& \frac{d s}{d t}=-\alpha s j,
\end{aligned}
$$

where the equation for recovered individuals is omitted because of conservation of the overall population $N=S+I+R$. Even in such a simple model no analytical solution can be given and one needs to rely on numerical solutions. Linear stability analysis near the zero fix point $j^{\star}=0, s=1$ shows that the epidemic takes place only if $\mathcal{R}_{0}=\alpha / \beta>1$. Here we have encountered the $\mathcal{R}_{0}$, " $\hbar$ of epidemiology" which gives the average number of secondary cases in completely susceptible population. Frequently, this number is uncritically used as a basic characteristic of the epidemic. However, the knowledge of $\mathcal{R}_{0}$ without structure of the intrinsic heterogeneity of the populations (Pastor-Satorras and Vespignani, 2000; May and 
Lloyd, 2001) is not enough to predict the outbreak of epidemics. It must be mentioned that the spatial structure of the population affects $\mathcal{R}_{0}$ as well, cf. (Arino and van den Driessche, 2003) and the discussion in Section 4.6. The threshold behavior characteristic for SIR is a common feature of different epidemiological models. To prevent an epidemic one needs to put the parameter under the outbreak threshold. The time course of the SIR epidemic is presented in Figure 2.3(b).

\subsubsection{SIS model}

SIS model or susceptible-infected-susceptible is a good model for non-severe diseases which do not cause immunity, such like some parasite or bacterial infections. Alongside with the reaction (2.16), recovery of infectives can occur which are not removed from the population contrary to the SIR case

$$
I \stackrel{\beta}{\rightarrow} S .
$$

SIS is essentially an extention of the SI model permitting a non-unity epidemic state $j^{\star}=$ $1-\beta / \alpha$ for $\alpha>\beta$ (otherwise there is no outbreak) and occasional extinction of the epidemic in the stochastic case. The solution of the mean-field equations reads

$$
j(t)=\frac{j_{0}(1-\beta / \alpha)}{\left(1-\beta / \alpha-j_{0}\right) e^{-(\alpha-\beta) t}+j_{0}} .
$$

For further details on different epidemiological models incorporating vital dynamics and social heterogeneity one can consult other books (Anderson and May, 1991; Murray, 1993; Keeling and Rohani, 2007; Daley and Gani, 1999; Diekmann and Heesterbeek, 2000). The three epidemiological models introduced above will be used in Chapter 4 to illustrate the spatial spread of infectious diseases due to recurrent host movements.

\subsection{Spatial spread of epidemics}

The epidemiological models as presented above describe epidemics in a single well-mixed population. This is only a very crude approximation if we want to describe the spatial spread of infectious diseases. Let us consider a set of populated locations or patches, i.e. the metapopulation (Hanski, 1998), and look precisely how the infectious disease spreads from one location to another. Directly transmitted infections are spread due to contacts between infected and susceptible individuals and thus the interlocation transfer of disease is mediated by movements of infected individuals between different locations. For a long time it was assumed that such movements have the character of random walk or diffusion (Rvachev and Longini, 1985; Hufnagel et al., 2004; Collizza et al., 2007). The individuals were considered as indistinguishable particles. The local dynamics could be described in such a case as e.g. for SI model

$$
\frac{d I_{n}}{d t}=\frac{\alpha}{N} S_{n} I_{n}+\sum_{m}\left(w_{n m} I_{m}-w_{m n} I_{n}\right),
$$




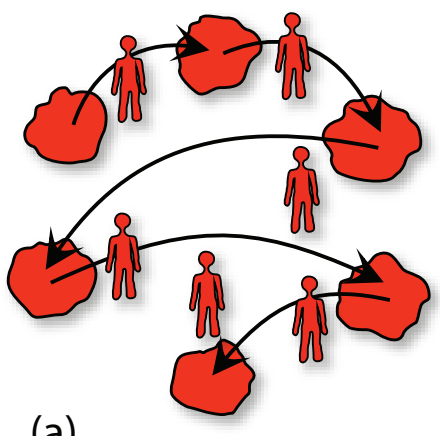

(a)

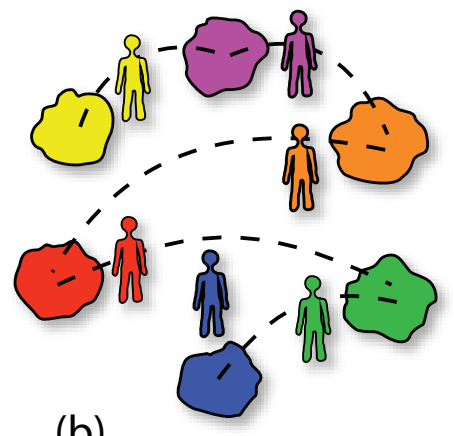

(b)

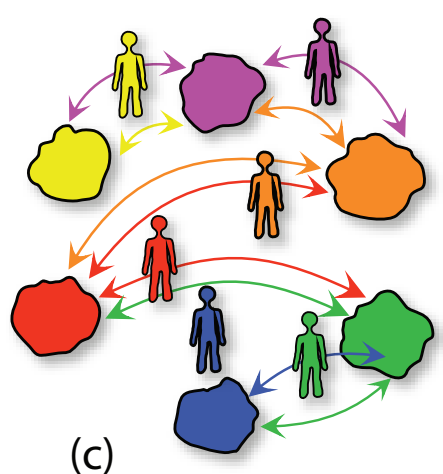

(c)

Figure 2.4: Schematic representation of three spatial epidemic models. (a) Reaction-diffusion model with infection spread due to random walk travel pattern of indistinguishable individuals. (b) Direct coupling model. Epidemics in different locations are heuristically coupled without explicit incorporation of the travel pattern. Dashed curves symbolize coupling. (c) Our model with epidemic spread mediated by the recurrent host movements between their abode and other locations with explicitly incorporated movement pattern. Colored arrows with symbolize movements of agents belonging to different locations.

where a term for the diffusive transport is added to the usual local infectious dynamics term. $w_{n m}$ are transition probabilities to move from location $m$ to location $n$, which can be directly assessed by measuring the flows between different locations. Thus this approach is called the reaction-diffusion approach. It is illustrated in Figure 2.4(a). The most genuine example of such situation are chemical systems (Ebeling and Sokolov, 2006). As a biological example one can mention foraging of animals (Edwards et al., 2008) which represents a prey-predator system with a spatial component. One can argue that historic nomadic populations have searched for food and new resources in a random walk manner. There were also individuals who spent a lot of time just wandering about, like pilgrims in the Middle Ages visiting religious sanctuaries or wandering Junggesellen in Germany. At the beginning of XX-th century in the US seasonal workers - hobos practiced a wandering way of life.

Often the transition to a continuum medium instead of discrete multi-patches description reveals essential insights into dynamics. In the continuum limit the reaction-diffusion approach leads to the Fisher-Kolmogorov-Petrovsky-Piscounov (FKPP) equation (Kolmogorov et al., 1937; Fisher, 1937) for the density of infectives $u=u(x, t)$

$$
\partial_{t} u=\mathcal{D} \Delta u+f(u)
$$

where $f(u)$ denotes a particular kind of infectious dynamics, e.g. for SI model $f(u)=\alpha u(1-$ $u) . \mathcal{D}$ is the diffusion coefficient. The advantage of the mean-field equation (2.19) and its continuum counterpart (2.20) is their derivation from first principles. The analysis of this model reveals the travelling wave pattern of the epidemic spread. An important analytically assessable quantity is the velocity of the wave front. This particular model was successfully applied to the spread of bubonic plague in the Medieval Europe (Noble, 1974). Let us note that if individuals are allowed to perform long-range movements, important consequences for 


\section{Fundamentals}

the spread of epidemics arise (Brockmann and Hufnagel, 2007). The role of disorder in such systems remains an important open question. We will consider it in Chapter 3.

In contrast to models assuming random walk movement pattern, hosts rather often possess some particular range of action, their abode or home range, i.e. the region where they spent most of the time leaving it only occasionally and return. Humans constitute the most pronounced example. Recently due to the availability of highly resolved data empirical evidence of recurrent and bounded or confined nature of human movements is established(González et al., 2008). In Figure 2.5(a) the probability $P(L)$ to be at the $L$-th preferred location is presented. One can observe that literally " $40 \%$ of the time individuals are found at their first two preferred locations". Thus humans spend their time mostly among the few locations belonging to their activity or home range. They often just commute between home and workplace and therefore can be hardly considered as indistinguishable particles performing random walk. If the home ranges of different individuals overlap, they can contact and infect each other and by returning to their home range contribute to the spatial spread of epidemics (Figure 2.5(b)). For some animals, the recurrent movement model constitutes also the appropriate description. Consider for example bees or ants.

Because the reaction-diffusion approach obviously does not work for humans and some animals, a heuristic model was proposed as early as in (Rushton and Mautner, 1955) which we call directly coupled model. In this model the incidence, i.e. the rate of change of the number of infectives in one location with time is proportional to the prevalence, i.e. the number of infectives in other locations. Proportionality factors or coupling strengths are supposed to be assessed a posteriori from past epidemics, without derivation from first principles. Individuals are distinguishable according to their home place. Host movements are not considered explicitly. In fact individuals do not move effectively at all and are considered as always present on all available locations. This model is illustrated in Figure 2.4(b). Until recently no connection of the coupling strengths to the movement properties were established. The mean-field equations for the directly coupled SI model read

$$
\frac{d I_{n}}{d t}=\alpha S_{n} \times\left(\sum_{m} \varepsilon_{n m} I_{m}\right),
$$

where $\varepsilon_{n m}$ denotes coupling strengths between location $m$ and location $n$. Such kinds of models were also successfully applied to the description of spatial spread of epidemics (Ferrari et al., 2008; Grenfell et al., 2001; Riley, 2007; Camitz and Liljeros, 2006). Coupling strengths were set to be proportional to travel rates $w_{n m}$ without justification in (Camitz and Liljeros, 2006).

A continuum formulation of the direct coupling approach was also investigated, e.g. in (Postnikov and Sokolov, 2007; Naether et al., 2008) A more general direct coupling model was proposed in (Lopez et al., 1999). The continuum equation in the SI case is

$$
\partial_{t} u=\alpha(1-u) \mathcal{D} \Delta u+\alpha u(1-u)
$$

with the coefficient $\mathcal{D}=\omega d^{2} / 2$ comprising the movement rate $\omega$ over inter-location distance $d$. Without establishing the connection with the recurrent movements it was found for example that the SIR wave front shape looks much more asymmetrical than it is predicted by the 

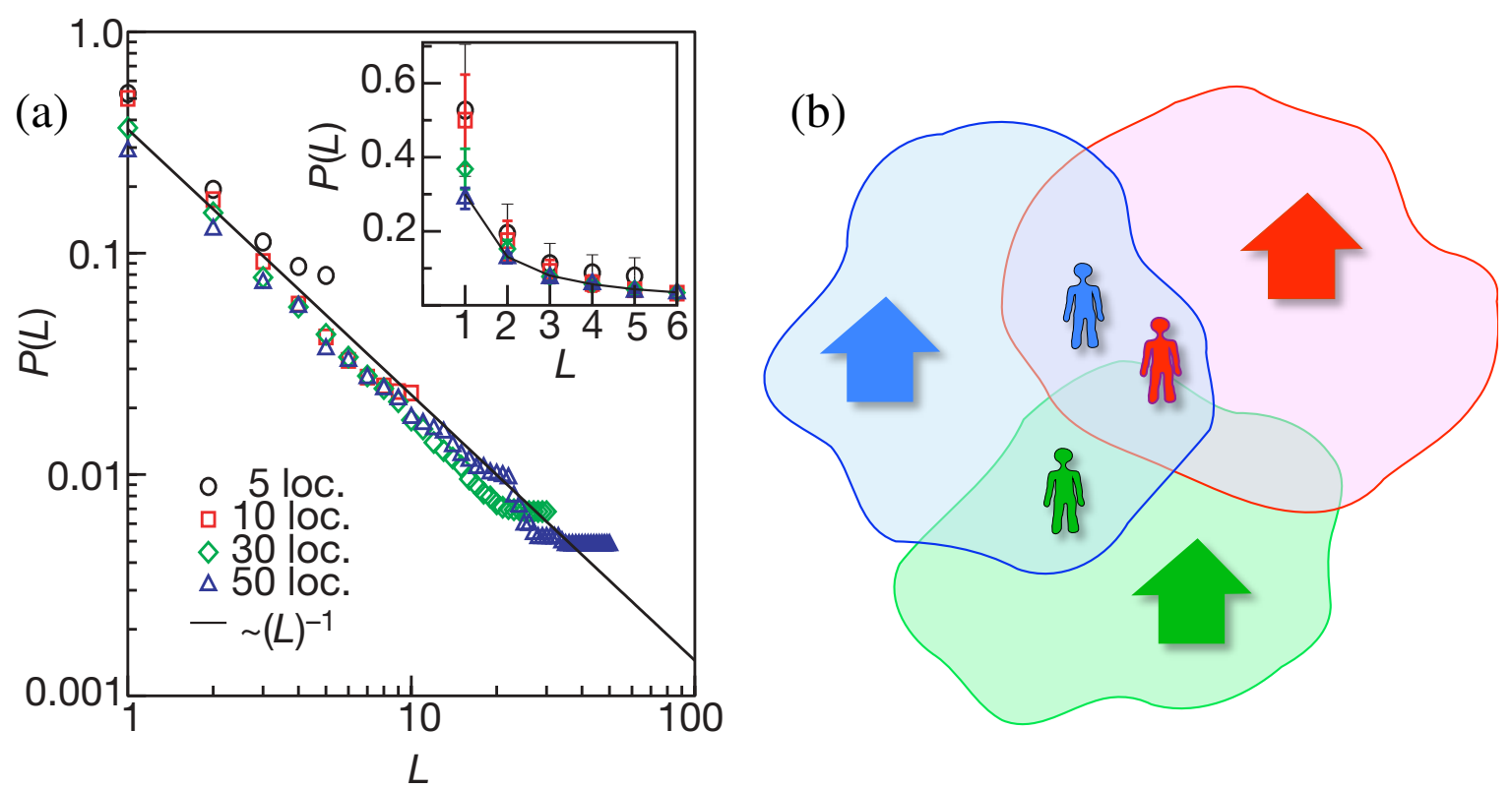

Figure 2.5: On panel (a) the probability $P$ to be on the $L$-th preferred location (first, second and so forth) is presented (González et al., 2008). Thus humans spend most of their time between a few locations within their range of activity. This presents empirical evidence for the recurrent nature of human movements. Panel (b) presents illustration of the overlap of individual activity ranges. The model for recurrent host movements will be analyzed in Chapter 4 .

reaction-diffusion model. Similar front shapes were indeed found empirically and are called Kendall waves. Compare also discussions in Section 4.4.6.

Our ultimate goal is to explicitly incorporate the recurrent movements into the model for the spatial spread of infectious disease. Chapter 4 is devoted to this topic and shows that the directly coupled model can be deduced from the model which we propose for description of epidemics with recurrent host movements only in the case of a high movement rate. Only in this case connection between coupling strengths and travel rate can be established (4.2). Otherwise to account for recurrent host movements one needs to consider a much more complicated model, the main idea of which is to subdivide individuals sojourning at some particular locations into subclasses according to their location of origin and then consider dynamics of these classes separately. This approach is illustrated in Figure 2.4(c). In the continuum limit in the case of the SI model with immobile susceptibles equations for epidemics due to recurrent movements read (Section 4.4.1)

$$
\begin{aligned}
\partial_{t} u & =\alpha(1-u-v)(u+v+D \Delta v)+\omega_{1} v-\omega_{2} u \\
\partial_{t} v & =\omega_{2} u-\omega_{1} v
\end{aligned}
$$

where $u=u(x, t)$ and $v=v(x, t)$ denote the number of infectives belonging to location $x$ being at home and elsewhere respectively. $\omega_{1}$ and $\omega_{2}$ denote forward (from home) and backward (to home) movement rates. $D$ is a constant incorporating interlocation distance. As one can observe, the equations describing the random walk model (2.20) and the recurrent movement (2.21) model are structurally different. It makes a difference if agents commute between 
determined location (their abode) or if they are allowed to travel among many different locations. We will show that one of the important consequences of the recurrent movement is a reduction of the velocity of the wave front as compared to random walk movement pattern (Section 4.4.2). Let us note that there were other attempts to go beyond ordinary random walks to properly account for movements of humans or animals, e.g. through the introduction of a finite jump velocity for random walks (Méndez et al., 2004), by considering a directed random walk (Horsthemke, 1999), by explicitly modeling overlapping home ranges (Reluga et al., 2006) or extensively simulating modern urban environments (Eubank et al., 2004).

Note, that for the models considered above we had in mind the epidemiological applications in the first place. Application in other fields of theoretical biology, such as prey-predator systems or evolutionary population dynamics, are obvious candidates for further research. 


\section{Balancing between start and destination: superdiffusion and gravity model}

As previously mentioned in Chapter 2, given two ingredients - the gravity model ansatz and the superdiffusive assumption for the movement rates of individuals - we recover the generalized topological superdiffusion model. In the present chapter, we define this model in a precise way. Performing theoretical and numerical model analysis, we will use powerful spectral tools such as the Bloch theory and perturbative method. We will demonstrate a striking difference in the qualitative behavior between ordinary diffusion and Lévy flights in inhomogeneous environment that reveals rather counterintuitive aspects of the generalized topological superdiffusion.

\subsection{The model}

Our general aim is to investigate the dynamic impact of heterogeneous environments on diffusive processes. We devote particular attention to the relative impact of source and target locations on the transition rates $w(\boldsymbol{x} \mid \boldsymbol{y})$ for spatial displacements of the random walk (see Figure 3.1). We define the spatial inhomogeneity of the environment in terms of the attractiveness or salience $s(\boldsymbol{x})>0$ of a location $\boldsymbol{x}$. For large and small values of $s(\boldsymbol{x})$, the location $\boldsymbol{x}$ is attractive and unattractive, respectively. We assume that in equilibrium, a walker's stationary probability $p^{\star}(\boldsymbol{x})$ of being at a location $\boldsymbol{x}$ is proportional to the salience at $\boldsymbol{x}$, i.e. $p^{\star}(\boldsymbol{x}) \propto s(\boldsymbol{x})$. In this respect, the salience field can be defined operationally as the attractiveness of a site $\boldsymbol{x}$. Furthermore, we assume that a transition from $\boldsymbol{y}$ to $\boldsymbol{x}$ is more likely to occur when the salience is large at the target location $\boldsymbol{x}$, and less likely to occur when the salience is large at the source location $\boldsymbol{y}$. This leads to a transition rate

$$
w(\boldsymbol{x} \mid \boldsymbol{y})=\frac{1}{\tau} s^{c}(\boldsymbol{x}) f(|\boldsymbol{x}-\boldsymbol{y}|) s^{c-1}(\boldsymbol{y}),
$$

where $\tau$ is a time constant and the sandwiched term $f(|\boldsymbol{x}-\boldsymbol{y}|) \propto|\boldsymbol{x}-\boldsymbol{y}|^{-(d+\mu)}$ with $0<\mu \leq 2$ accounts for Lévy flight jump lengths. $d$ denotes a spatial dimension and we need to care of normalization. Inserting (3.1) into the master equation

$$
\partial_{t} p(\boldsymbol{x}, t)=\int d y w(\boldsymbol{x} \mid \boldsymbol{y}) p(\boldsymbol{y}, t)-w(\boldsymbol{y} \mid \boldsymbol{x}) p(\boldsymbol{x}, t)
$$

one can see that the detailed balance is fulfilled such that the stationary solution, if it exists, is proportional to the salience. 


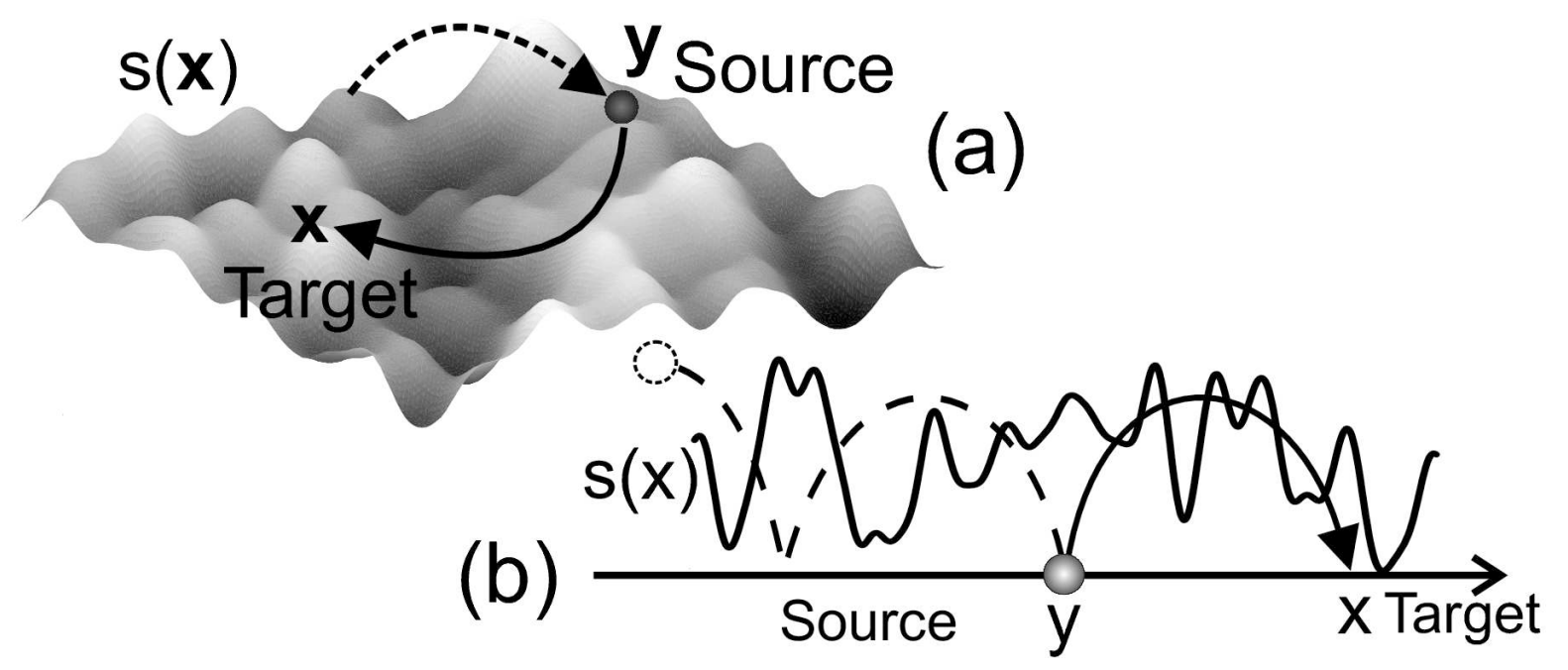

Figure 3.1: Random walk processes in inhomogeneous salience fields $s(\mathbf{x})$ in two (a) and one (b) dimension. Source and target locations of a single jump are denoted by $\mathbf{y}$ and $\mathbf{x}$, respectively.

The central parameter in our analysis is the weight parameter $0 \leq c \leq 1$, which quantifies the relative impact of source and target salience on the dynamics. When $c=1$, a transition $\boldsymbol{y} \rightarrow \boldsymbol{x}$ only depends on the salience at the target site and is independent of the salience at the source. When $c=0$, the salience of the target site has no influence on the transition, and the rate is decreased with increasing salience at the source. The intermediate case $c=1 / 2$, is equivalent to the topological superdiffusion approach (Brockmann and Sokolov, 2002) with a salience given by a Boltzmann factor $s(\boldsymbol{x})=e^{-\beta v(\boldsymbol{x})}$ in a potential $v(\boldsymbol{x})$.

In combination with the transition rate (3.1), the master equation (3.2) is equivalent to the fractional Fokker-Planck equation (FFPE)

$$
\partial_{t} p=D s^{c} \Delta^{\mu / 2} s^{(c-1)} p-D p s^{c-1} \Delta^{\mu / 2} s^{c},
$$

where $p=p(\boldsymbol{x}, t), s=s(\boldsymbol{x})$ and $D$ is a generalized diffusion coefficient. The fractional Laplacian is defined by

$$
\Delta^{\mu / 2} f(\boldsymbol{x})=\mathcal{C}_{\mu} \int d \boldsymbol{y} \frac{f(\boldsymbol{y})-f(\boldsymbol{x})}{|\boldsymbol{x}-\boldsymbol{y}|^{d+\mu}}
$$

with $\mathcal{C}_{\mu}=2^{\mu} \pi^{-d / 2} \Gamma((\mu+d) / 2) / \Gamma(-\mu / 2)$. In Fourier space, $\Delta^{\mu / 2}$ corresponds to multiplication by $-|\boldsymbol{k}|^{\mu}: \mathcal{F}\left\{\Delta^{\mu / 2} f\right\}(\boldsymbol{k})=-|\boldsymbol{k}|^{\mu} \mathcal{F}\{f\}(\boldsymbol{k})$. In what follows, we will restrict our analysis only to a one-dimensional case, although generalizations to further dimensions are straightforward. Note that in the case of ordinary diffusion Equation (3.3), reduces to the usual Fokker-Planck equation

$$
\partial_{t} p=\left[-\nabla F+\frac{1}{2} \Delta D\right] p,
$$

where $F(x)=-c \beta \frac{d v}{d x} D(x)$ and $D(x)=2 \exp [-\beta(2 c-1) v(x)]$.

The fractional Fokker-Planck equation (3.3) reduces to a number of known stochastic processes for specific choices of the parameters $c$ and $\mu$. For instance, when $s(x)=$ const (3.3) 
is equivalent to free superdiffusion, i.e. (2.9). When $c=1 / 2$ and $\mu=2$, (3.3) reads $\partial_{t} p=$ $-\nabla F p+\Delta p$, with an effective force $F(\boldsymbol{x})=-v^{\prime}(\boldsymbol{x})$ and the corresponding effective potential $v(\boldsymbol{x})=-\beta^{-1} \log s(\boldsymbol{x})$, i.e. diffusion in an external force field. When $c=0$, (3.3) reads $\partial_{t} p=$ $D \Delta^{\mu / 2} p / s$, which is equivalent to generalized multiplicative Langevin dynamics (Fogedby, 1998) for the process $X(t)$, i.e. $\mathrm{d} X=D(X) \mathrm{d} L_{\mu}$ with $D(X)=\sqrt{2} \exp [\beta V(X) / 2] / D$, and $L_{\mu}(t)$ is a homogeneous Lévy stable process (Feller, 1971). Note that $c=0$ corresponds to a well-known case of diffusion among random wells or traps (Haus and Kehr, 1987; Bouchaud and Georges, 1990; ben Avraham and Havlin, 2000).

As it is known, under certain conditions, the ordinary Fokker-Planck equation is equivalent to the Schrödinger equation used in quantum physics with an imaginary time $i \hbar t$ instead of $t$ (Risken, 1996). In the following, we will use a formulation of the problem in terms of the generalized fractional Schrödinger equation (FSE), which is an advantage because of the symmetry of the resulting operator. Indeed, the FFPE (3.3) is not symmetric because in general, the transition rate $w(x \mid y)$ is not equal to the rate of transition in the opposite direction $w(y \mid x)$. However, if the detailed balance condition is fulfilled and there exists a stationary solution of Equation (3.3), one can recast the original problem into symmetric form using a transformation

$$
p(x, t) \rightarrow \psi(x, t)=s^{1 / 2}(x) p(x, t) .
$$

Thus we arrive at the generalized fractional Schrödinger equation

$$
\partial_{t} \psi(x, t)=\mathcal{H} \psi
$$

where without a loss of generality we set $D=1$. The last equation reads explicitly

$$
\partial_{t} \psi=s^{c-1 / 2} \Delta^{\mu / 2} s^{c-1 / 2} \psi-\psi s^{c-1} \Delta^{\mu / 2} s^{c} .
$$

It has the same spectral properties as the original equation (3.3). This fact will be used in the folowing investigation of the generalized topological superdiffusion in disordered environments.

\subsubsection{Disordered environment}

Having formulated the problem of superdiffusion in inhomogeneous media, let us turn to the actual models of the media. Instead of the operational definition of the salience from the previous section, it can now be defined as the Boltzmann probability with a parameter $\varepsilon>0$ corresponding to inverse temperature and quantifying the strength of the inhomogeneity

$$
s(x)=e^{-\varepsilon v(x)},
$$

for a given potential $v(x)$, which we need to select now. Investigating different diffusive and superdiffusive phenomena in an external force field, the most elementary potentials are frequently considered, e.g the harmonic one - the simplest potential leading to a non-constant force. If we are interested in the transport properties of the diffusive processes like in the Kramer's escape problem, a double-well potential is a good choice. If we have in mind the motivation, i.e. the gravity model for movements of individuals, it does not seem to be of 
particular interest to consider the case of just one or two attractive locations modelled by oneor two-well potential. Considering e.g. animal foraging or human travel habits in population dynamic or epidemiological context, we are faced with inhomogeneous environments (be it a spatial distribution of resources, preys, or sizes of the habitats) in extended and complex form.

As it was shown (Brockmann, 2003), inhomogeneities with a finite variance do not scale out for the topological superdiffusion in contrast to the generalized Langevin approach (Fogedby, 1998). This holds for the generalized topological superdiffusion model as well. Indeed, performing a transformation of the spatial variable $x \rightarrow z=x / \gamma$ the fractional Schrödinger equation (3.4) reads ${ }^{1}$

$$
\partial_{t} \psi(\gamma z, t)=\frac{1}{\gamma^{\mu}}\left[s^{c-1 / 2}(\gamma z) \Delta^{\mu / 2} s^{c-1 / 2}(\gamma z) \psi(\gamma z, t)-\psi(\gamma z, t) s^{c-1}(\gamma z) \Delta^{\mu / 2} s(\gamma z)\right] .
$$

From the last equation, it is obvious that we can restore an invariant form of the fractional Schrödinger equation if we transform the time coordinate as $t \rightarrow \tau=t / \gamma^{\mu}$. The salience is defined as the Boltzmann probability (3.5) and the potential with finite variance does not vanish even on large scales.

For all values of the parameter $c \neq 1 / 2$, the transition rate defined as (3.1) depends on a potential offset. This implies that if we substitute the potential $v(x)$ by another one differing only by the constant $v_{0}>0$ from the old one, the effective time constant from the otherwise same transition rate will change as

$$
\tau \rightarrow \frac{\tau}{\exp \left[\varepsilon v_{0}(2 c-1)\right]}
$$

Thus, for $c<1 / 2$ (large influence of the source location) an increase of the potential leads to lower transition rates, while for $c>1 / 2$ this leads to higher transition rates as compared with the transition rate for the original potential.

For further investigations, we chose seven inhomogeneous model potentials - four periodic and three random ones - which may serve as a good approximation of real systems.

\subsubsection{Periodic potentials}

We chose periodic potentials as a first approximation of an inhomogeneous environment. We will see later that the main dynamical features and properties of the superdiffusive processes with dis-balanced role of the source and target locations, will be preserved also in the case of more complicated models of the environment, such as random potentials. Furthermore, periodic potentials are self-sappealing because of a powerful and beautiful method of Bloch bands which can be successfully applied in our case as well. Computational demands are also relatively easy to satisfy as compared with random potentials. More complex inhomogeneities may be considered as a superposition of periodic inhomogeneities with different periods (Economou, 2006).

As representative potentials, we chose the following four sample potentials (figure 3.2 and table 3.1). A - the most simple case with the only one harmonic in the Fourier representation

\footnotetext{
${ }^{1}$ We have used here the scaling property of the fractional Laplacian $\Delta_{\gamma z}^{\mu / 2}=\frac{1}{\gamma^{\mu}} \Delta_{z}^{\mu / 2}$.
} 


\begin{tabular}{|c|c||c|c|}
\hline & $v(x)$ & & $S(k)$ \\
\hline \hline $\mathbf{A}$ & $\sqrt{2} \cos (x / \lambda)$ & $\mathbf{E}$ & $2 \xi \theta(|k-\pi /(2 \xi)|)$ \\
\hline $\mathbf{B}$ & $\begin{array}{l}1 \quad \frac{2 n-1}{4}<x / \lambda<\frac{2 n+1}{4} \\
-1 \quad \text { otherwise }\end{array}$ & $\mathbf{F}$ & $2 \xi \exp \left(-\frac{k^{2} \xi^{2}}{\pi}\right)$ \\
\hline $\mathbf{D , C}$ & $\pm a[\cos (x / 2 \lambda)]^{2 \gamma}+b$ & $\mathbf{G}$ & $2 \xi \exp \left(-\frac{2 \xi|k|}{\pi}\right)$ \\
\hline
\end{tabular}

Table 3.1: Analytical expressions for periodic potentials (A-D) and spectra of the random phase potentials (E-G) used to model an inhomogeneous environment. Parameters $a$ and $b$ were chosen in a way to fulfill normalization requirements (3.6). Parameter $\gamma$ was set to 32. See also Figure 3.2.

is of course a cosine potential. As a coarse approximation of cosine, a piecewise potential $\mathbf{B}$ can be chosen. For sparsely distributed inhomogeneities, seldom peaks $\mathbf{C}$ and seldom wells $\mathbf{D}$ are good model potentials with opposite bias. All types of potentials are normalized so that they possess zero mean and unit variance

$$
\frac{1}{2 \pi \lambda} \int_{2 \pi \lambda} d x v(x)=0 \text { and } \frac{1}{2 \pi \lambda} \int_{2 \pi \lambda} d x v^{2}(x)=1
$$

\subsubsection{Random potentials}

If we bear real applications in mind, periodic inhomogeneities are of course an exaggeration and just a first approximation to real environments. If we aim to harness the understanding of realistic phenomena, there is no way around considering random environments. To model random environments, we have chosen four different random correlated potentials defined by the formula

$$
v(x)=\frac{1}{2 \pi} \int d k \phi(k) e^{i \theta(k)-i k x}
$$

where integration is performed over the whole infinite domain. Here the random phase $\theta(k)$ is uniformly distributed over the interval $(0,2 \pi]$ and the amplitude is given by the correlation spectrum $S(k)$, satisfying the relation

$$
\phi(k) \overline{\phi\left(k^{\prime}\right)}=2 \pi S(k) \delta\left(k-k^{\prime}\right)
$$

where the bar denotes complex conjugation. We chose three different spectra with the correlation length $\xi$, which are listed in Table 3.1. Potentials are depicted in Figure 3.2: $\mathbf{E}$ is a potential with a Heaviside power spectrum, $\mathbf{F}$ is a potential with a Gaussian spectrum and $\mathbf{G}$ possesses an exponential power spectrum. We also require, as in the case of periodic potentials, zero mean and unit variance

$$
\langle v(x)\rangle_{v}=0 \text { and }\left\langle v^{2}(x)\right\rangle_{v}=1 .
$$



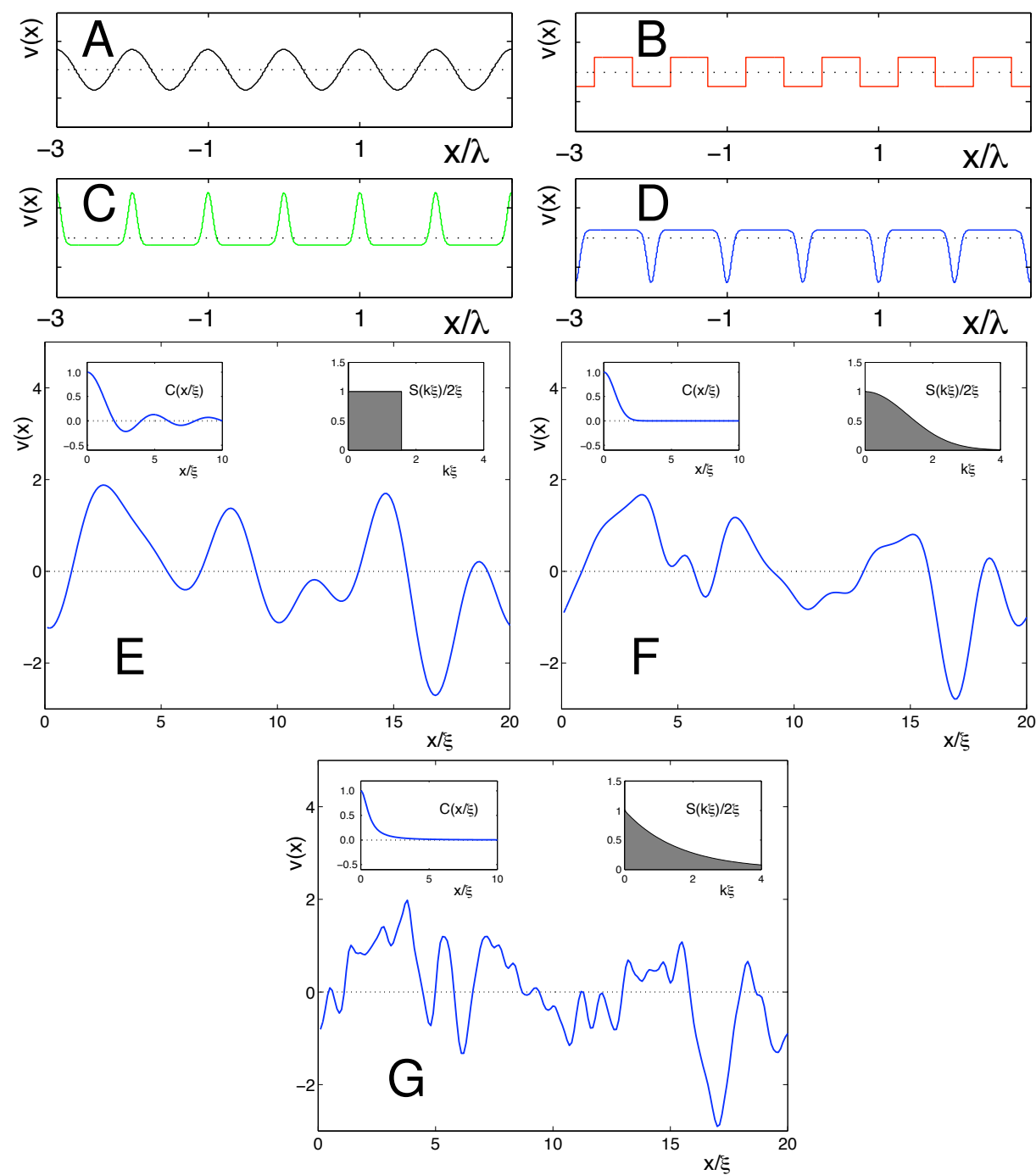

Figure 3.2: Potentials used to model inhomogeneities possessing internal length scale with zero mean and unit variance. Periodic potentials: A - cosine potential, B - piecewise constant potential, C and $\mathrm{D}$ - potentials with seldom inhomogeneities and opposites bias (peaks and wells respectively). Random potentials: E - potential with a Heaviside power spectrum, F - potential with a Gaussian power spectrum, $\mathrm{G}$ - potential with an exponential power spectrum. Insets show power spectrum $S(k)$ and correlation function $C(x)=\int d k e^{-i k x} S(k)$ of corresponding random potentials. See also Table 3.1.

We will develop an analytical perturbation theory for weak inhomogeneities in Section 3.2. Utilizing these analytical results, we will turn our attention to the dynamics of diffusive and superdiffusive processes on intermediate (Section 3.3) and large (Section 3.4) scales. We will compare our analytical results with the numerics in the case of strong inhomogeneities. Considering the dynamics on intermediate scales in periodic inhomogeneities, we will use a powerfull method of Bloch bands (Appendix A). Finally, we investigate the finite-size effects (Section 3.6), discuss the consequences of the salience normalization, resembling the evolu- 
tionary game theory (Section 3.7) and briefly mention how reaction can be incorporated in our model (Section 3.8).

\subsection{Perturbative analysis}

As mentioned in the previous section, the fractional Fokker-Planck equation (3.3) can be recast into the generalized fractional Schrödinger equation (3.4). To analyze this equation in the case of weak inhomogeneities we can use a powerfull tool - perturbation theory (Landau and Lifshitz, 2002). The main idea of it is as follows: given the salience in the form (3.5) we expand it in terms of the effective potential strength $\varepsilon \ll 1$ up to the second order, we obtain the Hamiltonian

$$
\mathcal{H} \approx \mathcal{H}_{0}+\mathcal{V}(\varepsilon)
$$

with an unperturbed free Hamiltonian $\mathcal{H}_{0}=\Delta^{\mu / 2}$ and the perturbation part given by (consult Appendix B)

$$
\begin{aligned}
& \mathcal{V} \psi=-\varepsilon\left[(c-1 / 2) \Delta^{\mu / 2} v \psi\right.\left.+(c-1 / 2) v \Delta^{\mu / 2} \psi-c \psi \Delta^{\mu / 2}\right] \\
&+\varepsilon^{2}\left[\frac{(c-1 / 2)^{2}}{2} \Delta^{\mu / 2} v^{2} \psi-(c-1 / 2)^{2} v \Delta^{\mu / 2} v \psi\right. \\
&\left.\quad+\frac{(c-1 / 2)^{2}}{2} v^{2} \Delta^{\mu / 2} \psi-\frac{c^{2}}{2} \psi \Delta^{\mu / 2} v^{2}-c(c-1) v \psi \Delta^{\mu / 2} v\right] .
\end{aligned}
$$

According to the stationary perturbation theory, the discrete part of the spectrum of $\mathcal{H}$ is given by

$$
E_{n}=E_{n}^{0}+V_{n n}+\sum_{m}^{\prime} \frac{\left|V_{m n}\right|^{2}}{E_{n}^{0}-E_{m}^{0}}
$$

with $E_{n}^{0}$ - eigenvalues of the unperturbed operator $\mathcal{H}_{0}$. The matrix elements $V_{n m}$ reads

$$
V_{n m}=\left\langle\varphi_{n}|\mathcal{V}| \varphi_{m}\right\rangle
$$

where $\varphi_{n}$ are orthogonal eigenfunctions of the unperturbed operator and we used the Dirac notation. e consider periodic and random potentials separately.

\subsubsection{Periodic potentials}

According to the Bloch theory (Appendix A) for periodic potentials, for which holds: $v(x+$ $2 \pi \lambda)=v(x)$, the spectrum of the Hamiltonian operator take the form

$$
E=E_{n}(q), \text { with } n \in \mathbb{Z}, q \in[0,1] .
$$

Due to continuity of the parameter $q$, called the Bloch phase we have a set of spectral bands numerated by the integer index $n$. 


\subsubsection{Non-degenerate energy levels}

If $q \neq 1 / 2$, the difference between energies of two levels $n \neq m$ of the free Hamiltonian is large $\left(E_{n}^{0}(q)-E_{m}^{0}(q) \sim \mathcal{O}(1)\right)$. It follows that the energies of the perturbed system can be expressed as

$$
E_{n}(q)=E_{n}^{0}(q)+V_{n n}+\sum_{m}^{\prime} \frac{\left|V_{n m}(q)\right|^{2}}{E_{n}^{0}(q)-E_{m}^{0}(q)},
$$

where matrix elements $V_{n m}(q)$ are given through $V_{n m}(q)=\left\langle\phi_{n}^{0}(q)\left|\mathcal{H}_{\text {pert }}(\varepsilon)\right| \phi_{m}^{0}(q)\right\rangle$. The plane waves $\phi_{n}^{0}(q)=e^{-i(n-q) x / \lambda}$ are eigenfunctions of the non-perturbed operator with periodic boundary conditions. Or equivalently

$$
V_{n m}(q)=\frac{1}{2 \pi \lambda} \int_{2 \pi \lambda} d x e^{-i(n-q) x / \lambda} \mathcal{V}(\varepsilon) e^{-i(m-q) x / \lambda} .
$$

In Section 3.4 we are interested in the dynamics on large scales. For that purpose, we will need to consider the first Bloch band $E_{0}(q)$. After some algebra we obtain from (3.8) (see Appendix B for analogous calculation in the case of random potentials)

$$
\begin{aligned}
\lambda^{\mu} E_{0}(q) & =-|q|^{\mu}-\varepsilon^{2} \sum_{m}\left[(c-1 / 2)^{2}|q|^{\mu}\left|\hat{v}_{m}\right|^{2}+(c-1 / 2)^{2}|m+q|^{\mu}\left|\hat{v}_{m}\right|^{2}\right. \\
& \left.-c(c-1)|m|^{\mu}\left|\hat{v}_{m}\right|^{2}-\frac{\left[(c-1 / 2)|q|^{\mu}+(c-1 / 2)\left(|m-q|^{\mu}-c|m|^{\mu}\right)\right]}{|m-q|^{\mu}-|q|^{\mu}}\right] .
\end{aligned}
$$

We restrict summation only to positive values and obtain

$$
\lambda^{\mu} E_{0}(q)=-|q|^{\mu}\left(1-2 \varepsilon^{2} \sum_{m>0} g_{\mu, c}(q / m)\left|\hat{v}_{m}\right|^{2}\right),
$$

where $g_{\mu, c}(z)$ is given by

$$
\begin{aligned}
g_{\mu, c}(z) & =-2(c-1 / 2)^{2}+\frac{1}{z^{\mu}}\left\{2 c(c-1)-(c-1 / 2)^{2}\left(|1-z|^{\mu}+|1+z|^{\mu}\right)\right. \\
& +\left[(c-1 / 2)\left(z^{\mu}+|1+z|^{\mu}\right)-c\right]^{2} /\left(|1+z|^{\mu}-z^{\mu}\right) \\
& \left.+\left[(c-1 / 2)\left(z^{\mu}+|1-z|^{\mu}\right)-c\right]^{2} /\left(|1-z|^{\mu}-z^{\mu}\right)\right\} .
\end{aligned}
$$

Thus, eigenvalues deviate from the free case by a factor proportional to $\varepsilon^{2}$, which also depends on the superdiffusive exponent $\mu$ as well as on the source-target impact $c$ and on the Fourier coefficients $\left|\hat{v}_{m}\right|^{2}$ of the potential.

We can introduce the generalized diffusion coefficient $D_{\mu, c}(q ; \varepsilon) \approx-E_{0}(q) /(q \lambda)^{\mu}$ to describe the influence of the inhomogeneity. In the limit of vanishing potential, the generalized diffusion coefficient is identical to the diffusion coefficient $D$ of the free system. Thus, from (3.9) we obtain

$$
D_{\mu, c}(q ; \varepsilon) / D=1-\varepsilon^{2} G_{\mu, c}(q)
$$

where

$$
G_{\mu, c}(q)=2 \sum_{m>0} g_{\mu, c}(q / m)\left|\hat{v}_{m}\right|^{2}
$$




\subsubsection{Energy levels near the Bragg plain}

If the Bloch phase takes values $q=0,1 / 2$ or 1 , the energies of the levels $\left(n_{1}, n_{2}\right):(n,-n),(1-$ $n, n)$ and $(n, 2-n)$ become degenerate and equation (3.8) diverges. We denoted as $n_{1}$ and $n_{2}$, levels with higher and lower energy (if approaching the Bragg plane from the left) of two degenerate in non-perturbed case levels. In this case, the correct equation reads (Nolting, 2000)

$$
E_{n}^{ \pm}(q) \approx E_{n}^{0}(q)+\frac{1}{2}\left(V_{n_{1} n_{1}}+V_{n_{2} n_{2}}\right) \pm \frac{1}{2} \sqrt{\left(V_{n_{1} n_{1}}-V_{n_{2} n_{2}}\right)^{2}+4\left|V_{n_{1} n_{2}}\right|^{2}}
$$

where, because of the condition $\left|V_{n_{1} n_{1}}-V_{n_{2} n_{2}}\right|=\mathcal{O}\left(\varepsilon^{2}\right) \ll \mathcal{O}(\varepsilon)=\left|V_{n_{1} n_{2}}\right|$, we can neglect a first term under the radical. Thus we obtain ${ }^{2}$

$$
\begin{aligned}
E_{n}^{ \pm}(q) & =E_{n}^{0}(q)-\frac{\varepsilon^{2}}{2 \lambda^{\mu}} \sum_{m}\left\{( c - 1 / 2 ) ^ { 2 } \left[2\left(\left|n_{1}-q\right|^{\mu}+\left|n_{2}-q\right|^{\mu}\right)+\left|n_{1}-m-q\right|^{\mu}\right.\right. \\
& \left.\left.+\left|n_{2}-m-q\right|^{\mu}\right]-2 c(c-1)|m|^{\mu}\right\}\left|\hat{v}_{m}\right|^{2}+\frac{\varepsilon^{2}}{\lambda^{\mu}} \sum_{m}\left[\frac { ( c - 1 / 2 ) ^ { 2 } } { 2 } \left(\left|n_{1}-q\right|^{\mu}\right.\right. \\
& \left.\left.+\left|n_{2}-q\right|^{\mu}+2|m|^{\mu}\right)+\frac{c^{2}}{2}\left|n_{1}-n_{2}\right|^{\mu}+c(c-1)\left|n_{2}-m\right|^{\mu}\right] \hat{v}_{n_{1}-m} \hat{v}_{m-n_{2}} \\
& +\frac{\varepsilon}{\lambda^{\mu}}\left[(c-1 / 2)\left(\left|n_{1}-q\right|^{\mu}+\left|n_{2}-q\right|^{\mu}\right)-c\left|n_{1}-n_{2}\right|^{\mu}\right] \hat{v}_{n_{1}-n_{2}} .
\end{aligned}
$$

\subsubsection{Random potentials}

In the case of random phase potentials we start with the finite support of the length $2 \pi L$. Then the eigenfunction of the unperturbed operator $\Delta^{\mu / 2}$ are plain waves $\phi_{n}=e^{-i n x / L}$ and thus the matrix elements are given by the expression

$$
V_{n m}=\left\langle\varphi_{n}|\mathcal{V}| \varphi_{m}\right\rangle=\frac{1}{2 \pi L} \int_{2 \pi L} d x e^{-i \frac{n}{L} x} \mathcal{V} e^{i \frac{n}{L} x}
$$

Proceeding in the same way as described in Appendix B, after the limit transition to the infinite support, we obtain the spectrum of our Hamiltonian in the form

$$
E(k) \approx-D_{\mu, c}(k ; \varepsilon)|k|^{\mu},
$$

where again the generalized diffusion coefficient $D_{\mu, c}(k ; \varepsilon)$, now for the case of random potentials, is introduced. The generalized diffusion coefficient describes the relaxation properties on the corresponding length scale $\Lambda=k^{-1}$. Again in the limit of vanishing potential the generalized diffusion coefficient is identical to the diffusion coefficient $D$ of the free system. Up to the second order in $\varepsilon$ we obtain

$$
D_{\mu, c}(k ; \varepsilon) / D=1-\varepsilon^{2} G_{\mu, c}(k),
$$

\footnotetext{
${ }^{2}$ With this equation one can explain the linear splitting of only lowest bands in cosine potential for $q=1 / 2$ (see Section 3.3). In this case $n_{1}-n_{2}=2 n-1$, and because all Fourier coefficients vanish except $2 n-1= \pm 1$, we have the linear splitting only in the lowest bands. For all other potentials, higher harmonics are present and we have linear splitting also in upper bands. Note that the splitting occurs in the higher bands for $c$ changing its values, apart of $c=1 / 2$, mostly due to terms proportional to $(c-1 / 2)^{2}$ (See Figures 3.3 and 3.7), a deviation from the linear splitting being also more pronounced.
} 
where

$$
G_{\mu, c}(k)=\frac{1}{2 \pi} \int d q S(q) g_{\mu, c}(k / q)
$$

with $g_{\mu, c}(z)$ given by (3.10) and integration performed over the whole infinite domain.

\subsection{Dynamics on intermediate scales}

In this section, we consider dynamics of the generalized topological superdiffusion on intermediate scales. We will pay particular attention to the influence of the source-target impact factor $c$. To this end, we will consider in detail the Bloch band structure for the periodic potentials and the dynamical factor $G_{\mu, c}(k)$ from (3.14) in the case of random potentials for different source-target impact values $c$. We will especially examine the differences of dynamical properties from the already-known balanced case $c=1 / 2$ (Brockmann and Geisel, 2003a,b). Generally, with varying $c$, differences between diffusive and superdiffusive case are more pronounced than differences among different superdiffusive processes. In what follows, we consider ordinary diffusive processes and superdiffusive processes separately. We will limit our analysis to the following choices of source and target impact factor $c=0,1 / 4,1 / 2,3 / 4,1$. We will compare ordinary diffusion $(\mu=2)$ with an exemplary superdiffusive case $(\mu=1)$. It will be shown that some intermediate-scale features are absent on large scales.

\subsubsection{Periodic potentials}

After numerical treatment of Equation (3.4), or more precisely (A.7), with potentials depicted in Figure 3.2, we note the following main features of the results. The structure of Bloch bands become richer and more involved as compared to the case of the balanced source-destination impact Brockmann and Geisel (2003a,b). Presented results can be immediately generalized to processes in random environments.

Because values of the energy are different quantities for different values of the power law exponent $\mu$, it is more convenient, instead of eigenvalues of the fractional Hamiltonianto, to consider the generalized crystal momentum, defined through

$$
\kappa_{n}(q)=\lambda\left[-E_{n}(q)\right]^{1 / \mu} .
$$

In the case of free motion it has the same value for all superdiffusive processes, namely

$$
\kappa_{n}(q)=|n-q| \quad \forall \mu: 0<\mu<2 .
$$

With the changing Bloch phase $q$ from zero to one-half, the crystal momentum changes continuously from minimum $\kappa_{n}(0)$ to maximum $\kappa_{n}(1 / 2)$ in each band. Thus, if we consider a dependence of the Bloch band structure on the potential strength $\varepsilon, \kappa_{n}(0)$ and $\kappa_{n}(1 / 2)$ are the only values we need, because all other eigenvalues are within these range. In the following we skip denoting the Bloch phase in the generalized crystal momentum as consider it a function of only the inhomogeneity strength, i.e. $\kappa_{n}(\varepsilon)$ bearing in mind that the Bolch phase $q$ takes all values from 0 to 1 . 
The most common features of a band structure are shrinkage, upward or downward shifts and appearance (disappearance) of gaps with varying $\varepsilon$. In what follows, qualitative interpretations of the band behavior will depend on one property of the exponential. An inequality $\int_{a}^{b} e^{-\lambda t} d \lambda<\int_{a}^{b} d \lambda e^{-(a+b) t / 2}$ holds (concavity of exponential) for modes with eigenvalues $\lambda$ in the range $[a, b]$ in the spectral decomposition of the solution of the Schrödinger equation (the separation ansatz). Hence, the shrinkage of bands (without shift) leads to the slowdown of the corresponding process. Here, we have compared decomposition sums with modes in the whole range $[a, b]$ and those with the only eigenvalue $\langle\lambda\rangle=(a+b) / 2$, given a uniform density of eigenvalues. The shift can be interpreted more easily. Downward shift means lower $\lambda$ values and thus slowdown of a process. Upward shift means higher $\lambda$ values and thus acceleration. Gaps can be reduced to shrinking and shift. An interplay between the above-mentioned factors determines dynamical properties of the system on corresponding scales.

\subsubsection{Ordinary diffusion}

Processes with Lévy index $\mu=2$ correspond to the ordinary diffusion case. As compared with the plain picture in the balanced case $c=1 / 2$, the band structure becomes more complicated. For example, gaps absent for $c=1 / 2$, appear. In general, all bands get thinner with growing $c$. Downward shift of the upper bands for extreme processes $c \rightarrow 0$ and $c \rightarrow 1$ implies attenuation on intermediate scales. The first band still decreases with $\varepsilon$, but for smaller $c$ it is broader, than for the intermediate $c$ values. It is thinner for larger $c$, implying stronger attenuation of processes with greater $c$ values (strong target impact) on large scales. Hence, dynamics on large and intermediate scales exhibits pronounced differences to the balanced case. While the behavior of the ordinary diffusion was not very much affected by the particular potential shape in the balanced case, we now see well-pronounced differences.

\subsection{Cosine potential}

The Bloch band structure obtained by the numerical diagonalization of the discrete version of the Hamiltonian for the ordinary diffusion process in the cosine potential (A) is presented in Figure 3.3. One can observe that, as compared to the case $c=1 / 2$, gaps appear in the upper bands and at the smaller inhomogeneity strength. Going away from values of $c$ around one half, the bands become thinner and shift downwards, and more upper bands become involved. However, for large extreme values of $c \rightarrow 1$, the effect is more pronounced, than for the small extreme values $c \rightarrow 0$. As it will be seen later, this effect is universal and appears in all potential types. This can be interpreted as follows: the downwards shift of the upper bands and simultaneous shrinkage for the extreme processes $(c \rightarrow 0, c \rightarrow 1)$ implies attenuation on intermediate scales (corresponding eigenvalues $\lambda_{n}$ become smaller) with increasing $\varepsilon$. This effect becomes even stronger in the superdiffusive case (cf. Section 3.3.1.2) and is also apparent in the case of random potentials (see Section (3.3.2)). Besides, more gaps and shrinkage of bands means the corresponding modes becomes almost $q$-independent.

Insights into dynamics on large scales discussed in detail in Section 3.4 can be found in the first band, which still decreases with $\varepsilon$, as it was also the case for $c=1 / 2$. However, for 


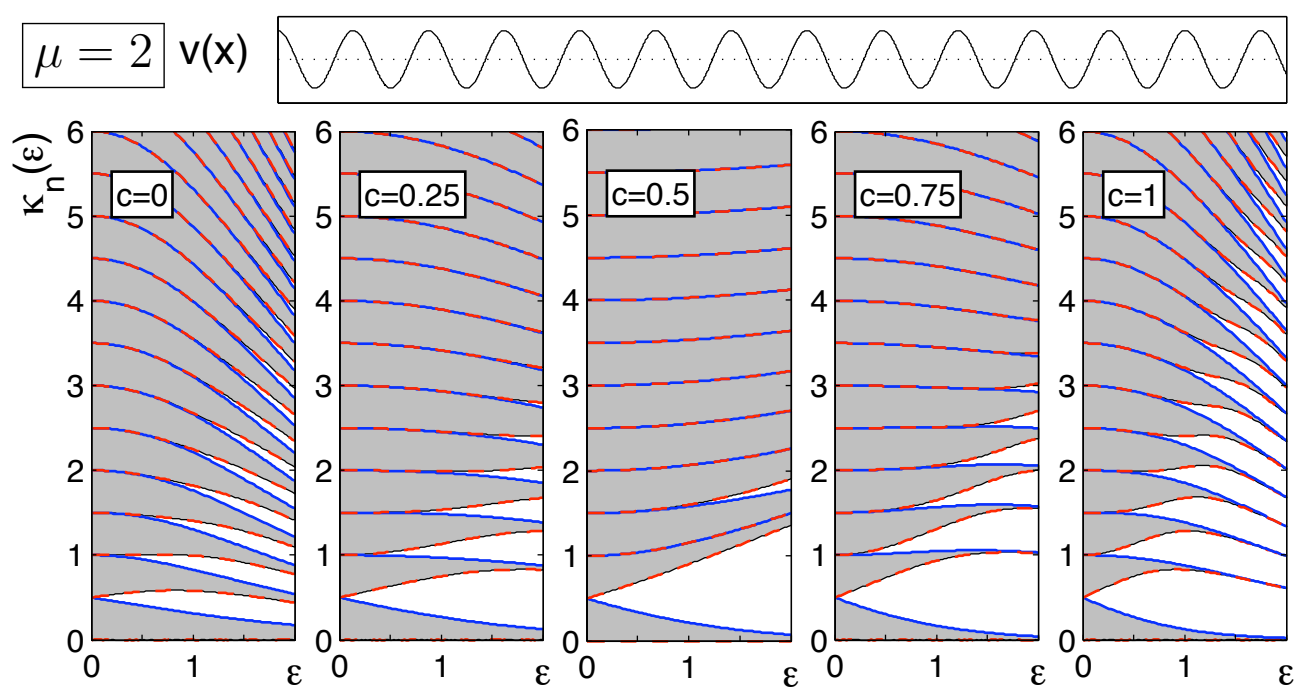

Figure 3.3: Ordinary diffusion in the cosine potential. The generalized crystal momentum $\kappa_{n}(\varepsilon)$ defined by Equation (3.15) is presented as function of effective potential strength $\varepsilon$. Panels depict band structures for different source-target impact $c=0,1 / 4,1 / 2,3 / 4$ and 1 . Blue and red lines correspond to upper and lower band boundaries.

smaller $c$, the band is broader, than for intermediate $c$ values, and is thinner for large values of $c$. That means less pronounced attenuation on larger scales for processes with strong source impact $(c<1 / 2)$ and more pronounced attenuation for processes with strong target impact $(c>1 / 2)$. Apart from this, one can conclude that, for very small and very large $c$, the overall band structures look pretty similar.

Note, that in the case of cosine potential the Fourier coefficients from (A.7) can also be obtained analytically in terms of the modified Bessel functions $J_{n}(\varepsilon \sqrt{2})$ with matrix elements of the Hamiltonian given by

$$
\langle n|\tilde{\mathcal{H}}(q)| m\rangle=\lambda^{-\mu} \sum_{l}\left[J_{m-l}(\varepsilon)|m-l|^{\mu} J_{l-n}(\varepsilon)-J_{m-l}(\varepsilon)|l-q|^{\mu} J_{l-n}(\varepsilon(c))\right]
$$

\subsection{Piecewise potential}

In contrast to smooth and plain behavior of the bands in cosine potential with varying potential strength $\varepsilon$, the band structure of the piecewise potential (B) looks much more complicated (Figure 3.4). A complicated interplay of many modes in the Fourier expansion, due to nonsmoothness of the potential shape, leads to this picture. Bands meet and repel each other. This kind of behavior in the balanced case was characteristic exclusively of superdiffusion. Thus, ordinary diffusion is now also very much affected by the piecewise potential for $c \neq 1 / 2$.

Dynamics on intermediate scales has some symmetry with regard to the value $c=1 / 2$ as was also the case for the cosine potential. Initially, starting at $c=0$, a complicated structure 


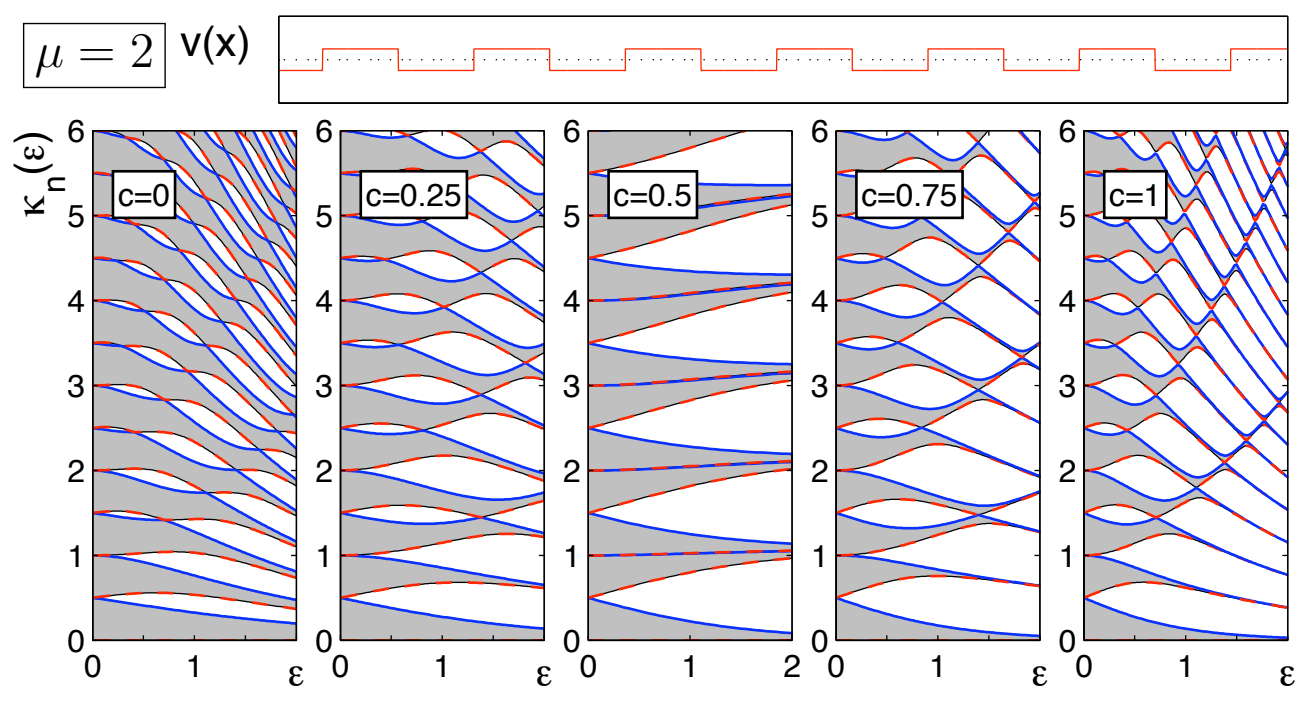

Figure 3.4: Ordinary diffusion in the piecewise constant potential. The generalized crystal momentum $\kappa_{n}(\varepsilon)$ as a function of effective potential strength $\varepsilon$ for different source-target impact $c$. For detailed description see caption of Figure 3.3. Note complex gaps for $c \neq 1 / 2$.

with many gaps becomes simpler. For $c=1 / 2$, one observes a smooth shrinkage of bands with growing $\varepsilon$ without gaps. With $c$ continuing to grow, gaps appear again and the picture becomes complicated, resembling the case $c=0$. The only remaining difference is the shrinkage of bands with $c$. Thus, ordinary diffusive processes on intermediate scales are least affected by the piecewise potential if $c=1 / 2$, i.e. equal source and target impact.

The first band looks rather similar to that for the cosine potential. This is also confirmed by the large scale asymptotic analysis (see Section 3.4). It becomes thinner with growing target impact, implying the most attenuation in the case $c=1$.

Note also, the similarity between the band structure of the piecewise and peaks potential for mostly target dependent processes as well as band structure in the wells potential for mostly source dependent processes. This implies the pronounced role of peaks and wells for mostly target and source dependent processes correspondingly.

\subsection{Peaks and wells potentials}

In more sophisticated potentials with seldom inhomogeneities ( $\mathbf{C}$ and $\mathbf{D}$ - peaks or wells, which differ only in their bias), an interesting effect appears. Namely, an a priory local ordinary diffusive process with $c \neq 1 / 2$ can "feel" the bias of the potential, i.e. can distinguish between peaks and wells potentials, although bands look identical in the balanced case $c=1 / 2$ (Figure 3.5 and 3.6). The further away the value $c=1 / 2$, the greater the difference. Thus, even in the ordinary diffusive limit, the potential bias has important implications for the band structure.

For the strongly target-dependent processes $(c \rightarrow 1)$ in the peaks potential, the band structure exhibits complex gaps, which vanish and appear again with growing $\varepsilon$ (See Figure 3.5), 


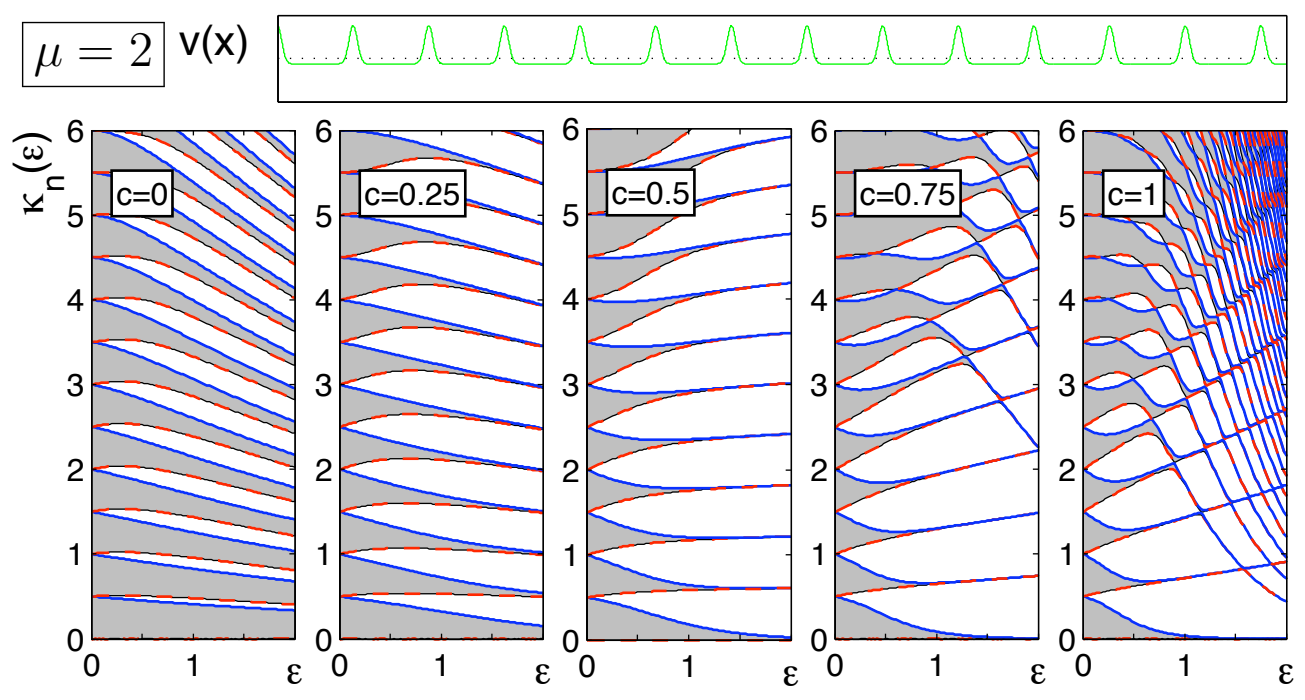

Figure 3.5: Ordinary diffusion in the potential with seldom peaks. The generalized crystal momentum $\kappa_{n}(\varepsilon)$ as a function of effective potential strength $\varepsilon$ for different source-target impact $c$. For detailed description see caption of Figure.3.3. Note the difference from Figure 3.6 in disbalanced case: $c \neq 1 / 2$.

witnessing a strong influence of inhomogeneity. A similar picture, though with broader bands that indicate less attenuation with a similar downward shift, emerges also in the wells potential in the case of strongly source-dependent process $(c \rightarrow 0$ Figure 3.6). Target-dependent processes in the peak potential get stuck in the attractive valleys like source-dependent processes in the wells potential get stuck in the wells. We could conclude that on intermediate scales, a process with $c \rightarrow 0$ in the wells potential relaxes faster than in the peaks potential for $c \rightarrow 1$, although both processes have similar properties. The same is also true on large scales, for which only the first band is significant. A further analysis will show that it is a general property of ordinary diffusive processes independent of the potential shape.

The only source dependent process $(c=0$ Figure 3.5$)$ does not feel peaks potential very much, as well as only target-dependent process in the wells potential ( $c=1$ Figure 3.6), because bands change smoothly with growing $\varepsilon$. In the peaks potential for $c=0$, only the source position determines the transfer rate. Almost all the locations are attractive and the probability to leave a location is relatively small. In the peaks potential, the source-dependent process seems to even be facilitated by unattractive peaks, leaving them with a higher probability. In the wells potential, and for $c=1$, almost all locations are non-attractive. There is also a large probability to leave even an attractive seldom well due to solely target dependence of the transfer probability. The bandwidth of upper bands, but not the shift, remains approximately the same for $c>1 / 2$ in the wells potential, implying that the process remains slightly affected by varying $c$ above one half. With growing target dependence, the probability to leave to an unattractive plateau of the well potential becomes higher, but not high enough to reduce attenuation. In both cases, bands have a slight downward shift and shrinkage of bands with $\varepsilon$ implying that the processes are more attenuated on intermediate scales, as compared to the balanced case. However, the source-dependent process in seldom peaks is less attenuated than 


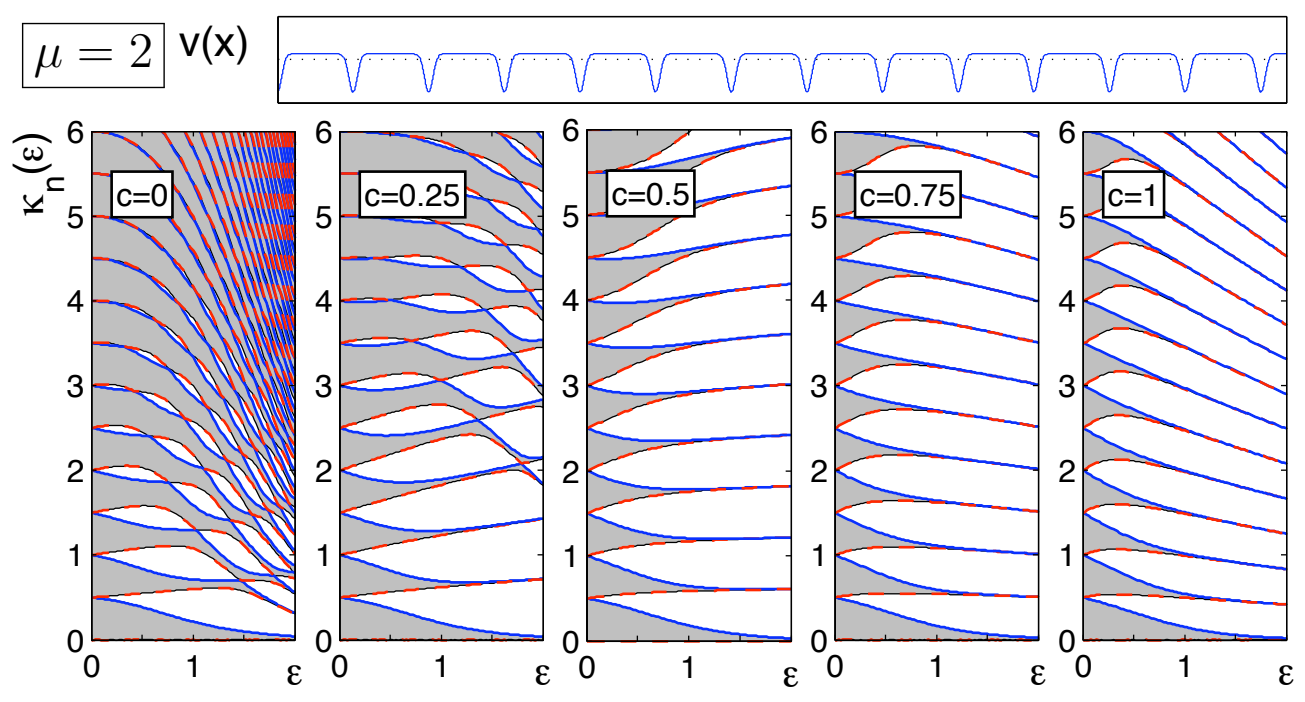

Figure 3.6: Ordinary diffusion in the potential with seldom wells. The generalized crystal momentum $\kappa_{n}(\varepsilon)$ as a function of effective potential strength $\varepsilon$ for different source-target impact $c$. For detailed description see caption of Figure 3.3. Note the difference from Figure 3.5 in disbalanced case: $c \neq 1 / 2$.

a target-dependent one in the wells potential. This is also indicated by broader bands in the only source dependent case in the peaks potential, than in the only target dependent case in the wells potential.

The first band does not change significantly with $c$ in the wells potential though it clearly becomes thinner in the peaks potential with growing $c$. Thus, in the last case, the process is even more slowed down on large scales in accordance with numerical results of the next section.

Let us conclude that even in the ordinary diffusion limit $\mu=2$, the potential shape has important implications for the band structure. Thus, essentially local ordinary diffusive processes can feel a bias of inhomogeneities, i.e. distinguish between wells and peaks potentials. The difference appears for $c$-values, moving apart from $c=1 / 2$, i.e. disbalancing equal source and target impact. In the ordinary diffusive case, the processes are still mostly slowed down with $\varepsilon$, especially on large scales. Attenuation of the processes becomes even stronger with a more definite target impact or, equivalently, growing $c$. This is no more the case for superdiffusion (cf. Section 3.3.1.2). On intermediate scales, the balanced diffusive process seems to be less slowed down in comparison to other source target impacts. These conclusions are supported by theoretical analysis in the case of ordinary diffusion in random potentials (Section 3.3.2).

\subsubsection{Superdiffusion}

Let us now consider Bloch bands for Lévy flights processes. All complicated band features such as gaps and shifts, present in the ordinary diffusive case, also remain present in the superdiffusive one. Furthermore, new features appear. The differences in the bands structure, as compared with the balanced case $c=1 / 2$, become even more amazing.

The most significant difference occurs in the first band. For all kinds of potential, the 

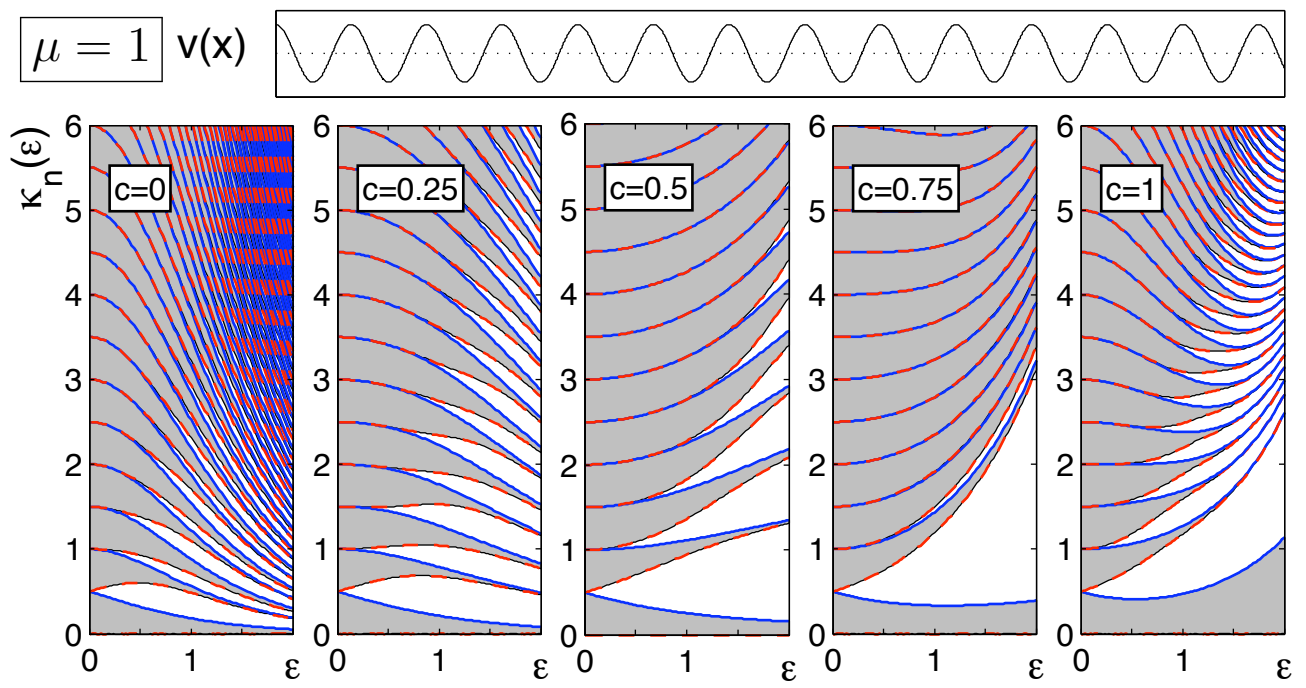

Figure 3.7: Superdiffusion with Lévy exponent $\mu=1$ in the cosine potential. The generalized crystal momentum $\kappa_{n}(\varepsilon)$ is presented in dependence on effective potential strength $\varepsilon$. Panels depict band structures for different source-target impact $c=0,1 / 4,1 / 2,3 / 4$ and 1 . Blue and red lines correspond to upper and lower band boundaries.

first band initially shrinka with $\varepsilon$, but broadens for larger $c$. This implies enhancement of processes on scales much larger than a wavelength of the potential. This is counter-intuitive to the common expectation that inhomogeneity attenuates diffusive processes. The effect is most pronounced in the wells potential. We investigate asymptotic dynamics on large scales in detail later in Section 3.4. Another interesting new aspect is an upward shift of the upper bands for higher $c$ values. This takes place in each potential type only in the superdiffusive case, implying enhancement on intermediate scales as well. Contrary to ordinary diffusion in all potentials, upper bands, initially shifted downwards for small $c$ values, experience an upward shift above some source-target impact $c>1 / 2$. However, the upward shift replaces an initially downward shift and begins only after some high enough value of the potential strength $\varepsilon$. For small $c$, and thus strong source impact, the downward shift, together with band shrinkage, leads to stronger attenuation.

\subsection{Cosine potential}

Again similar to ordinary diffusion, in the cosine potential bands of superdiffusive processes look plain without complex gaps for all $c$ values (Figure 3.7). Contrary to the ordinary diffusion case (Figure 3.3), instead of the smooth shift of upper bands first downwards for small $c$, then upwards for intermediate $c$ and finally again downwards for large $c$, we observe a continuous shift, first downwards, then upwards with varying/ $c$. However, for small $\varepsilon$ a downward shift is still present. The upward shift evidences the attenuation of the process on corresponding scales. As already mentioned, this feature is characteristic for superdiffusive process in all potentials, but is most pronounced in the cosine potential. 


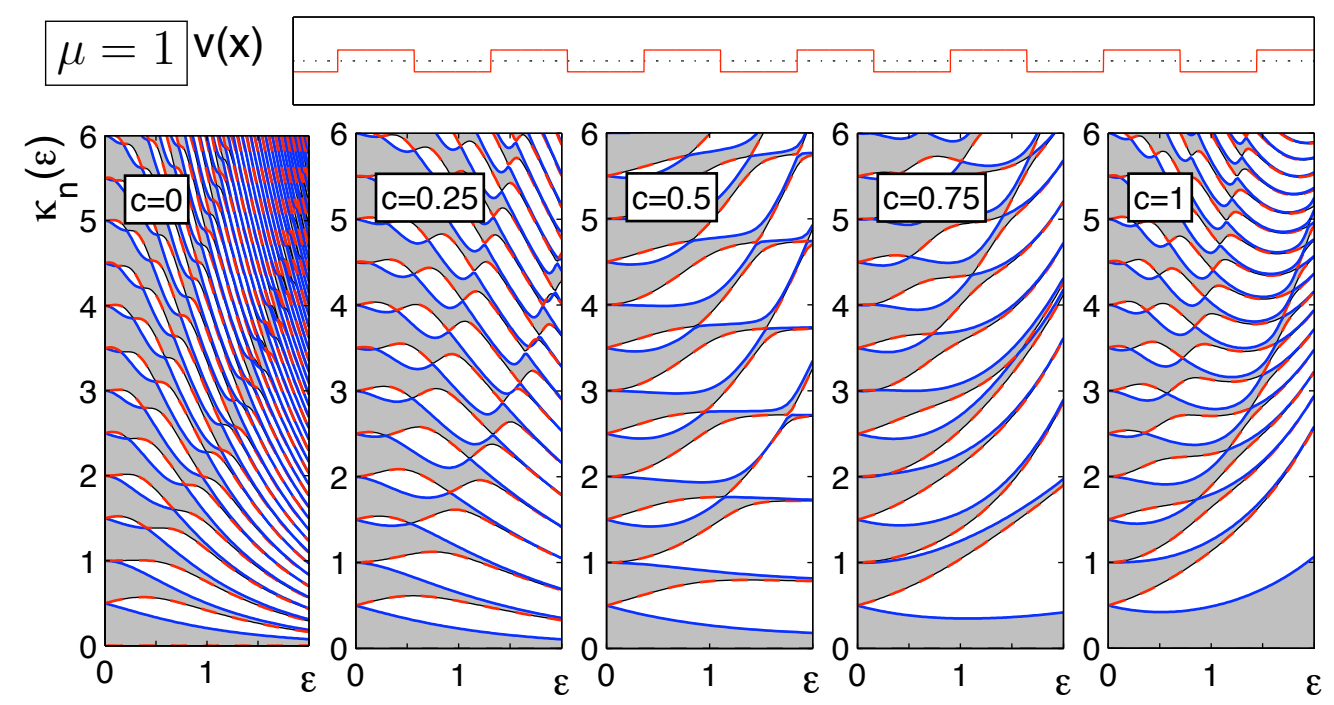

Figure 3.8: Superdiffusion with Lévy exponent $\mu=1$ in the piecewise potential. The generalized crystal momentum $\kappa_{n}(\varepsilon)$ in dependence on effective potential strength $\varepsilon$. For detailed description consult Figure 3.7.

One can observe a collapse of upper bands for small $c$ values which was not present in the ordinary diffusive case. Thus, the strongly source-dependent superdiffusive processes are substantially affected by the cosine potential. A less pronounced dense-band region appears for large $c$ as well. This was not the case for ordinary diffusion in the cosine potential. The collapse is even more noticeable in more complex potentials (Section 3.3.1.2.3).

\subsection{Piecewise constant potential}

One can observe that for all $c$ values, complex gaps are present in the band structure (Figure 3.8). Besides complex gaps, the overall picture is very similar to the cosine potential. We observe a collapse of upper bands for small $c$ values, and their upward shift for $c \rightarrow 1$ after an initial downward shift for small values of $\varepsilon$. The first band broadens in a way very similar to the cosine case, implying that dynamics on large scales are almost indistinguishable in both potentials. If we compare bands of the piecewise potential with the band structure of peaks and wells potentials (Figure 3.11 and Figure 3.12), there seems to be more similarity with the band structure of the wells potential for large $c$, than with the picture for peaks potential and small values of $c$. This implies that regions of high attractiveness (wells) become very important in the superdiffusive case with peaks playing almost no role. In the ordinary diffusion case (Figure 3.4), influence of peaks and wells in the upper bands was more balanced.

Some symmetry in the band structure in respect to $c=1 / 2$ present in the ordinary diffusion case, is not present anymore. Slight deviations from the balanced source-target impact $c=1 / 2$ on both sides lead to different band structures. A stronger target impact $(c>1 / 2)$ leads to 
a rather plain picture and a stronger source impact $(c<1 / 2)$ leads to complex bands. Thus, corresponding processes can be more or less affected by the piecewise potential.

For the special case of piecewise constant potential, along with the numerical diagonalization of the discretized Hamiltonian in the Fourier space (A.7), we can calculate Bloch bands structure by means of the much simpler Kronig-Penney (KP) method (de L Kronig and Penney, 1931; Ashcroft and Mermin, 1976). It is based on standard quantum mechanical consideration of tunneling through a potential barrier. Unfortunately, this method fails in the case $c>0$ for superdiffusive processes, may be due to non-local properties of superdiffusion. Note that this method is applicable to the usual Schrödinger equation, and the case $c=0$ leads to the common Schrödinger equation, describing diffusion in wells (Haus and Kehr, 1987; ben Avraham and Havlin, 2000). However, it is enough, as long as we need the KP method to confirm the anomalous collapse of higher bands in case $c=0$. We will observe that band collapse is still present and constitutes an intrinsic dynamical feature.

3.3.1.2.2.1 Kronig-Penney method The Schrödinger equation (3.4) for two regions of constant potential (Figure 3.9(a)) can be solved independently and the solutions should be sewed smoothly at the boundary. To this end we consider (A.1) for $c=0$

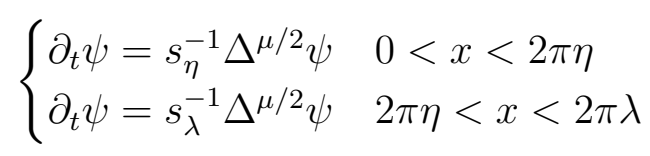

with $s_{\eta}=e^{-\varepsilon v_{\eta}}$ and $s_{\lambda}=e^{-\varepsilon v_{\lambda}}$. Making an ansatz (incident and reflected plane waves)

$$
\begin{cases}\psi=B e^{i \beta x}+B^{\prime} e^{-i \beta x} & 0<x<2 \pi \eta \\ \psi=A e^{i \alpha x}+A^{\prime} e^{-i \alpha x} & 2 \pi \eta<x<2 \pi \lambda,\end{cases}
$$

using the Bloch theorem $\psi(x)=e^{-i q x / \lambda} \theta(x)$, requiring periodicity and continuity of the wave function and its derivative on the boundary, the generalized crystal momentum ought to fulfill

$$
\cos (2 \pi q)=F(\kappa)
$$

where

$$
F(\kappa)=\cos (2 \pi \beta \eta) \cos [2 \pi \alpha(\lambda-\eta)]-\frac{\alpha^{2}+\beta^{2}}{2 \alpha \beta} \sin (2 \pi \beta \eta) \sin [2 \pi \alpha(\lambda-\eta)]
$$

and $\alpha=\kappa e^{\varepsilon V_{\lambda} /(2 \mu)} /(2 \pi \lambda)$ and $\beta^{2}=\kappa e^{\varepsilon V_{\eta} /(2 \mu)} /(2 \pi \lambda)$. The dependence $\kappa(\varepsilon)$ can be found numerically from the last equation, and for this purpose there is an illustration in Figure 3.9. Since cosine cannot be larger than unity by the absolute value, there are forbidden zones each time $|F(\kappa)|>1$. Bands obtained by solving the equation (3.17) numerically are presented in Figure 3.10(b). To access the usefulness of the Kronig-Penney model, we present here the results for ordinary diffusion. The similarity is striking (cf. Figure 3.4 and Figure $3.10 c=0$ ). Peaks and wells potential discussed in the next section can be considered as extreme cases of the piecewise potential with $\eta / \lambda \ll 1$ and $\lambda / \eta \ll 1$ respectively. Corresponding bands are also depicted in Figure 3.10. As expected, they are very similar to bands obtained by the diagonalization of the Hamiltonian (Figure 3.11 and Figure $3.12 c=0$ ). 


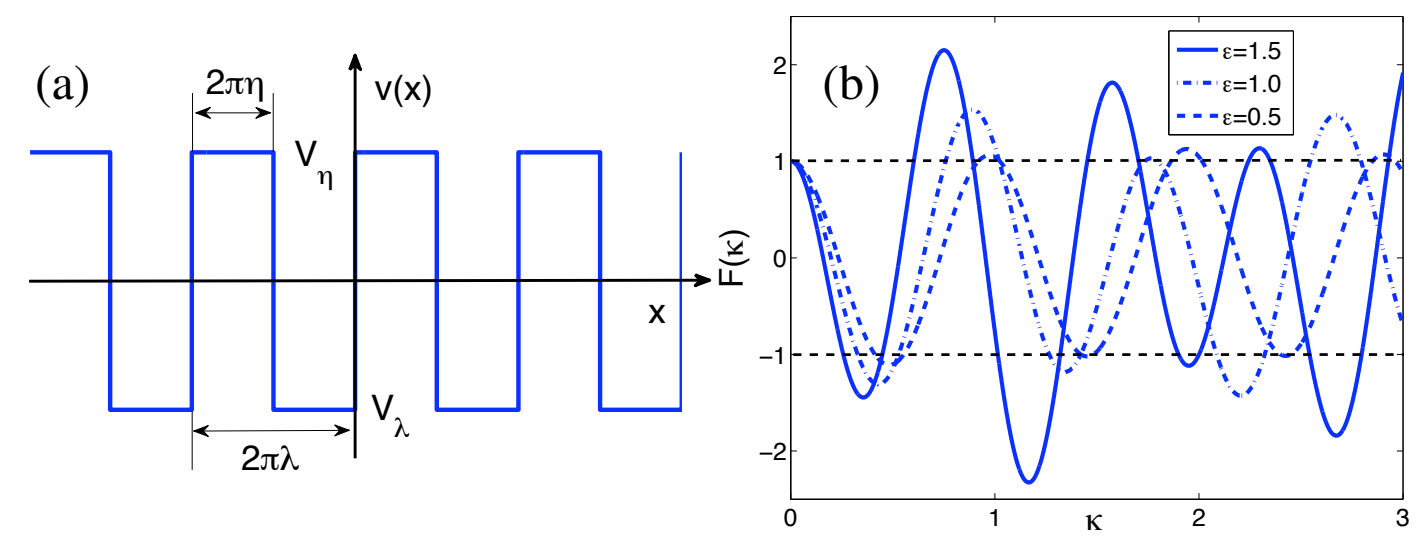

Figure 3.9: (a): Piecewise potential with parameters for the Kronig-Penney model. Allowed values of generalized crystal momentum $\kappa_{n}(\varepsilon)$ lie within regions determined by a condition on the function $|F(\kappa)| \leq 1$ defined by Equation.(3.18). (b): $F(\kappa)$ for different effective potential strength $\varepsilon$ and Lévy exponent $\mu=1$ is presented. $\eta / \lambda=0.5$.

3.3.1.2.3 Wells and Peaks potentials If seldom inhomogeneities are present, again, as in the ordinary diffusive case, complex gaps appear for strongly source-dependent processes in the wells potential and for strongly target-dependent processes in the peaks potential (Figure 3.11 and 3.12). On the other hand for strongly source dependent processes in the peaks potential ( $c=0 ; 1 / 4$ Figure 3.11) dependence on $\varepsilon$ is also smooth as well as for strongly target-dependent processes in the wells potential (Figure $3.12 c=3 / 4 ; 1$ ). However, in the latter case, upper bands are shifted upwards. In the former case they are shifted downwards. Thus, a difference to the ordinary diffusion consists in the upwards shift for strongly targetdependent processes, implying acceleration on corresponding scales.

In the peaks potential and strongly source dependent case, the downward shift of upper bands for superdiffusive processes is more pronounced as compared to the ordinary diffusion (Figure 3.11 and Figure 3.5). Thus, in the peaks potential source-dependent processes are strongly attenuated on intermediate scales in the superdiffusive case. In the only targetdependent case of the peaks potential ( $c=1$, Figure 3.11), a smooth behavior of the lower bands with changing $\varepsilon$, and a complicated structure of the upper ones, indicates that superdiffusion on large scales is not affected by inhomogeneity to such an extent as on intermediate scales. The complex band structure is present on intermediate scales as opposed to all scales of analogous ordinary diffusion case (Figure 3.5). The sharp downward shift can also be found in upper bands with the same implication on corresponding scales. Compared with the ordinary diffusive case, this dense gap structure (Figure 3.11 and Figure 3.5) persists for larger $c$ values. A similar picture, though with broader bands on all scales and a collapse of upper bands for large $\varepsilon$ values, appears also in the wells potential in the case of the mostly source-dependent process ( $c=0$ Figure 3.6), indicating attenuation on corresponding scales. Compared with the ordinary diffusion, the attenuation arises for larger $c$ values (cf. $c=0$ Figure 3.12 and Figure 3.6). Broader bands (without additional shift) generally imply less attenuation as compared with thinner bands (see discussion on page 31). For processes with small $c$ and $\varepsilon$ values, 

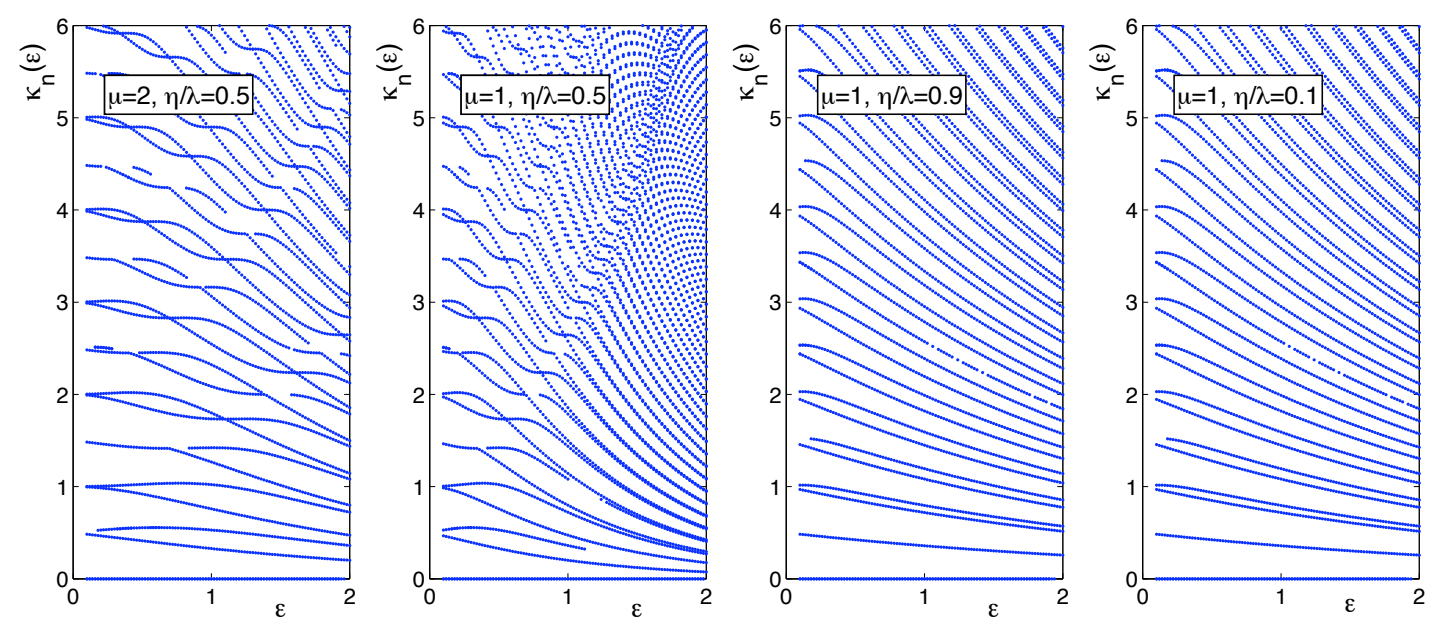

Figure 3.10: Band structures obtained by the Kronig-Penney method in the only source-dependent case $c=0$. Two right panels correspond to ordinary diffusive and superdiffusive $\mu=1$ processes in symmetric piecewise potential $\eta=\lambda$ (see fig.3.9). Two left panels correspond to superdiffusion $(\mu=1)$ in asymmetrical piecewise potential with opposite bias. Note the amazing similarity to the band structures of peaks $(\eta / \lambda=0.1)$ and wells $(\eta / \lambda=0.9)$ potentials $(c=0$ Figure 3.11 and Figure 3.12). Only upper and bottom boundaries for each band are plotted.

gaps emerge in lower bands, indicating a slow-down on large scales. In an intermediate range of $\varepsilon$ values, a fast downward shift of upper bands and band collapse can be observed. Thus, on corresponding scales, the attenuation is enhanced by a downward shift and band shrinkage (for $c=0$ ). What concerns the dynamics of strongly target dependent processes on large scales, if we take the present upward shift into account, we conclude that the most acceleration occurs in the wells potential as compared to the cosine and piecewise potentials. The peaks potential accelerates superdiffusive processes to the least extent. These findings are also confirmed in Section 3.4.

Let us summarize our qualitative analysis of the band structure. In contrast to ordinary diffusion, the first band becomes broader with source-target impact strength $c$ in the superdiffusive case. This implies a counterintuitive acceleration of such processes on large scales. Acceleration happens after some critical value of the target impact $c>1 / 2$. Thus, for a superdiffusive process to be enhanced, it is not sufficient to have only some prevalence of the target over the source impact. The prevalence should be strong enough. This observation is fully confirmed by perturbation analysis in the next section. On intermediate scales, we also have more pronounced acceleration for large $c$ values as compared with ordinary diffusion. Generally, one frequently observes the collapse of upper bands for very small and very large $c$ values. This indicates hindering of diffusion on corresponding scales. Not only superdiffusive processes but also ordinary diffusive ones, can distinguish between potential with opposite bias if source and target impacts are disbalanced. In general, sensitivity of the processes to a potential shape depends on the source-target impact. Presented qualitative insights into the dynamics on large scales will be compared with the analytical perturbation results and numerical diagonalization of the generalized Schrödinger equation (A.2) in the case of weak potential strengths $(\varepsilon \rightarrow 0)$ 


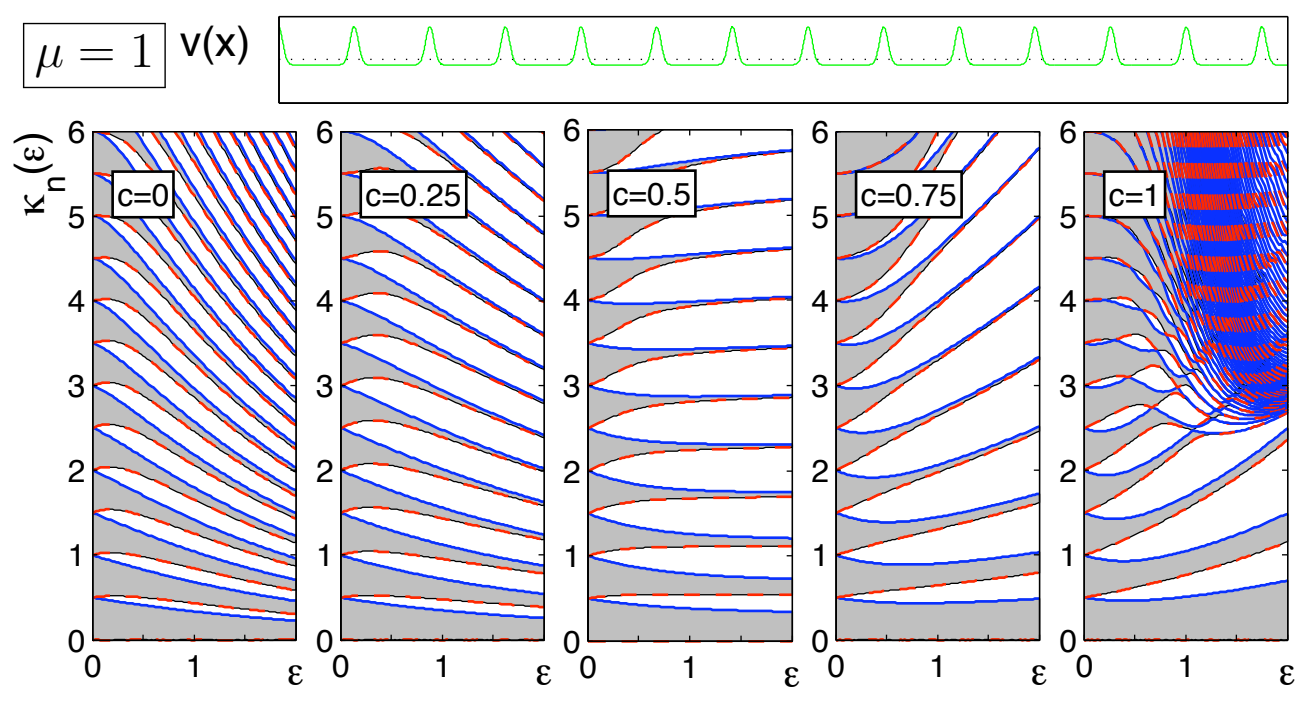

Figure 3.11: Superdiffusion with Lévy exponent $\mu=1$ in the potential with seldom peaks. The generalized crystal momentum $\kappa_{n}(\varepsilon)$ is presented in dependence on effective potential strength $\varepsilon$. For detailed description consult Figure 3.7.

in Section 3.4.

\subsubsection{Random potentials}

In the previous section we considered behaviour of the generalized superdiffusive processes in periodic potentials on intermediate scales. In the present section, we will draw our attention to the dynamics on intermediate and small scales in random potentials. According to the results of perturbative analysis (Section 3.2), the generalized diffusion coefficient is given by

$$
D_{\mu, c}(k ; \varepsilon) / D=1-\varepsilon^{2} G_{\mu, c}(k) .
$$

The function $G_{\mu, c}(k)=2 \int_{0}^{\infty} d q S(q) g_{\mu, c}(k / q)$ gives us insight into the dynamics on the corresponding spatial scale $x \sim 1 / k$. We will restrict oneselves to the case of Gaussian correlation spectrum $(\mathbf{F})$. Behavior in potentials with other correlation spectra reveals essentially the same features. The dependence of $G_{\mu, c}(k)$ on $k$, measured in the inverse units of the correlation length of the potential $\xi$ for different $c$ values and different Lévy exponents $\mu$, is presented in Figure 3.13 for ordinary diffusion and superdiffusion ${ }^{3}$. Three regimes can be distinguished: a small scale regime, an intermediate regime on scales approximately equal to the correlation length, and a large scale regime, which will be the topic of Section 3.4. We discuss ordinary diffusive and superdiffusive cases separately.

\footnotetext{
${ }^{3}$ Note that due to the scaling argument from Section 3.1 we can always rescale our coordinates so that $\xi=1$ without altering the properties of the system.
} 


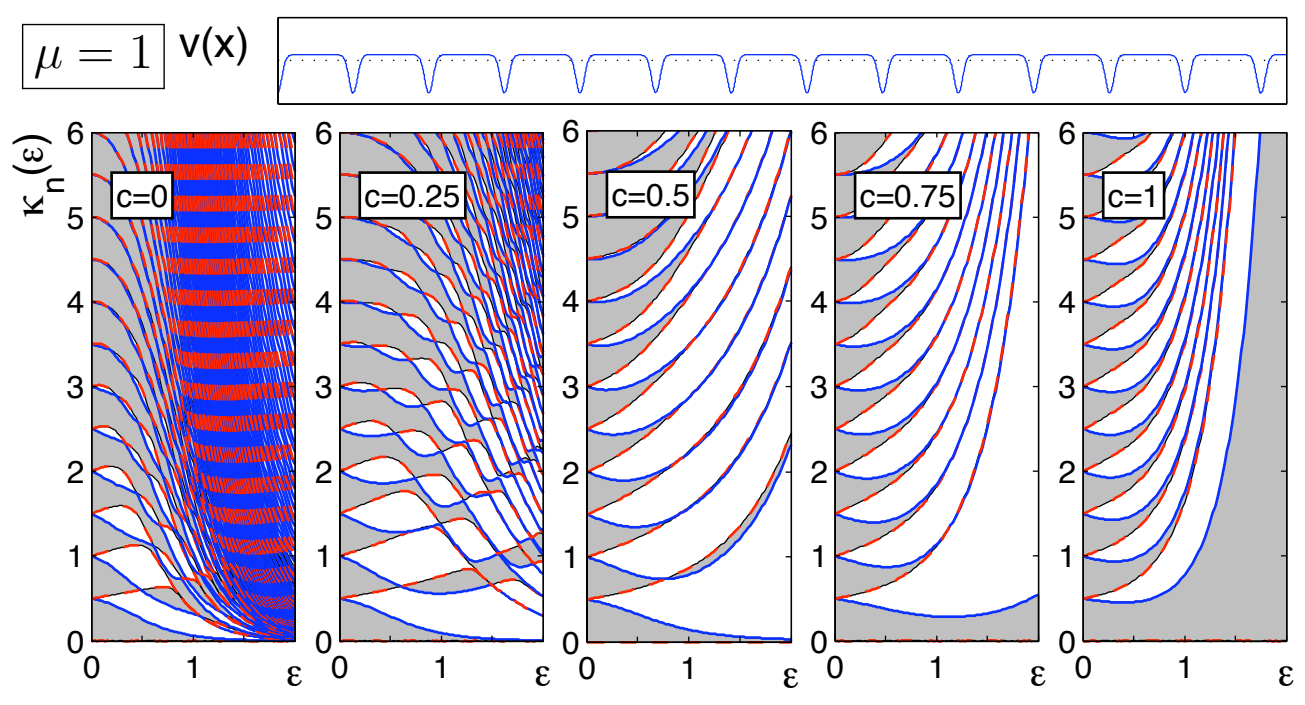

Figure 3.12: Superdiffusion with Lévy exponent $\mu=1$ in the potential with seldom wells. The generalized crystal momentum $\kappa_{n}(\varepsilon)$ is presented in dependence on effective potential strength $\varepsilon$. For detailed description consult Figure 3.7.

\subsubsection{Ordinary diffusion}

With a varying source-target impact in the ordinary diffusive case, a general course of the dependence $G_{2, c}(k)$ changes only quantitatively; no new global or local maxima appear. Large scale $(k \rightarrow 0)$ and small scale $(k \rightarrow \infty)$ limits are attained from below. The overall variation of the factor $G_{2, c}(k)$ is maximal for $c=1$ and minimal for $c=0$.

The small scale asymptotics does not monotonously depend on $c$. For the extreme $c$ values (strongly source- and target-dependent processes), $G_{2, c}(k)$ witnesses a stronger attenuation because it is larger, than in the balanced case $c=1 / 2$, in which $G_{2, c}(k) \sim 0$. The small scale asymptotics is the largest and the same for $c=0$ and $c=1$, implying that the processes have the same relaxation properties on corresponding scales. Note, that the small scale asymptotics does not vanish as was in the case for the balanced transfer rate $c=1 / 2$, in which the support on small scales becomes homogeneous. In the case $c \neq 1 / 2$ the fractional Fokker-Planck $\mathcal{L}$ operator (3.3) depends on the potential offset for $c \neq 1 / 2: \mathcal{L}\left[v(x)+v_{o}\right]=e^{(2 c-1) \varepsilon v_{o}} \mathcal{L}[v(c)]$. The small scale limit of $G_{\mu, c}(k)$ can be calculated as a product of $\frac{1}{2 \pi} \int d q S(q)=1$ and $\lim _{z \rightarrow \infty} g_{\mu, c}(z)$ :

$$
\lim _{k \rightarrow \infty} G_{\mu, c}(k)=\frac{1}{2 \mu}(2 c-1)^{2} .
$$

The dependence of $G_{\mu, c}(k \rightarrow \infty)$ on $\mu$ and $c$ is illustrated in Figure 3.14(a). Hence, the smaller $\mu$ values lead to greater attenuation on small scales. On the other hand, the more balanced the source-target impact is, the more $\mu$-independent the small scale asymptotics becomes. For $c=1 / 2$, it is completely $\mu$-independent and equals zero.

In the intermediate regime $1 / k \sim \xi, G_{2, c}(k)$ is positive for small $c$ values; with growing $c$ it becomes smaller. After some threshold value $c^{\star}<1 / 2, G_{2, c}(k)$ becomes negative and attains 

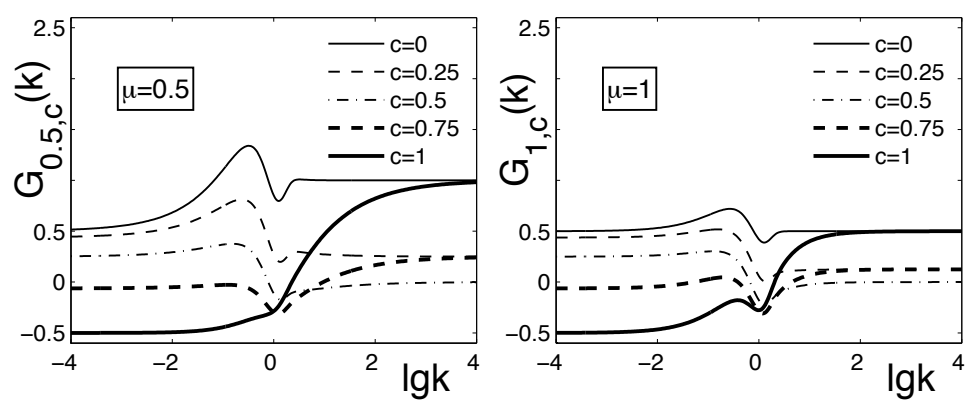

Figure 3.13: Dependence of the factor $G_{\mu, c}(k)$ determining the impact of the random phase potential
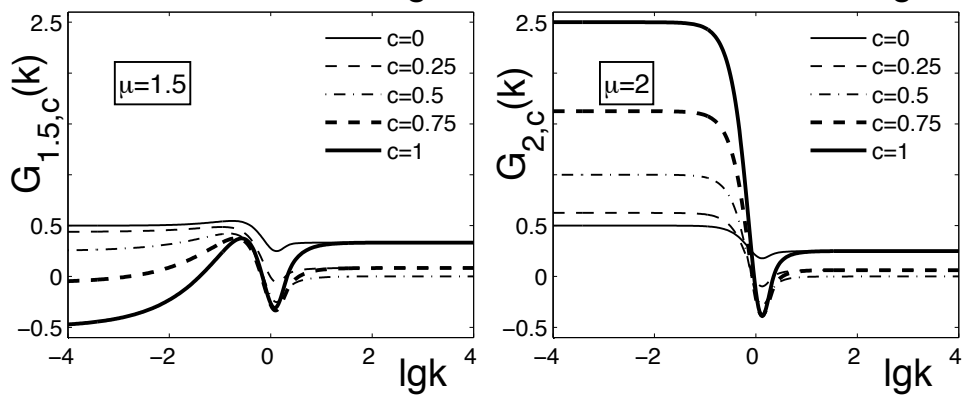
with a Gaussian correlation spectrum on diffusive processes with different Lévy exponents $\mu=$ $0.5 ; 1 ; 1.5$ and 2.

its local minimum which becomes most pronounced for $c=1$. Negative values of $G_{2, c}(k)$ correspond to the enhancement of the process even in the diffusive case, but only for some range of $c$ values. This stands in contrast to the balanced case where negative values of $G_{\mu, c}(k)$, i.e. acceleration, occurs only for superdiffusion (Brockmann, 2003). We can approximately calculate the dependence $c^{\star}(\mu)$ from the condition $g_{\mu, c}(1) \approx 0$ using the definition of $g_{\mu, c}$ (3.10). Hence, we obtain

$$
c^{\star}(\mu)=1-\sqrt{1-2^{-\mu}} .
$$

This dependence is presented in Figure 3.14(b). The smaller the Lévy exponent $\mu$, the larger the source-target impact is needed to obtain acceleration on the scales comparable with the characteristic scales of the inhomogeneity. The value of $c=1 / 2$ (balanced case) corresponding to the already obtained Lévy exponent $\mu_{t h} \approx 0.415$ (Brockmann, 2003) is depicted by the red circle.

\subsubsection{Superdiffusion}

The dependence of $G_{\mu, c}(k)(0<\mu<2)$ in the superdiffusive case, characterizing the transient dynamics, looks very different, as compared with ordinary diffusion. Depending on the $c$ values, the curves look very distinct, most changes occurring for $c>1 / 2$ (compare Figure 3.13 for $\mu=0.5$ with $c=1 / 2$ and $c=1$ ). For $c<1 / 2$, starting from small values of the argument, the curves go above their positive large scale asymptotic limit, reach the global maximum, then go down, reach the global minimum and then attain their small scale asymptotics from below. With increasing $c$ after certain threshold value $c_{\text {crit }}$ the form of the curves changes qualitatively ${ }^{4}$. It starts now from its negative large scale asymptotic limit, goes up, reaches its local maximum (except the strongly superdiffusive case, $\mu=0.5$ ), then goes down for a while, reaches its local minimum and then goes up again, reaching their positive

\footnotetext{
${ }^{4}$ As we will see in Section 3.4, the threshold value of source-target impact is $c_{\text {crit }}=1 / \sqrt{2} \approx 0.7071$.
} 

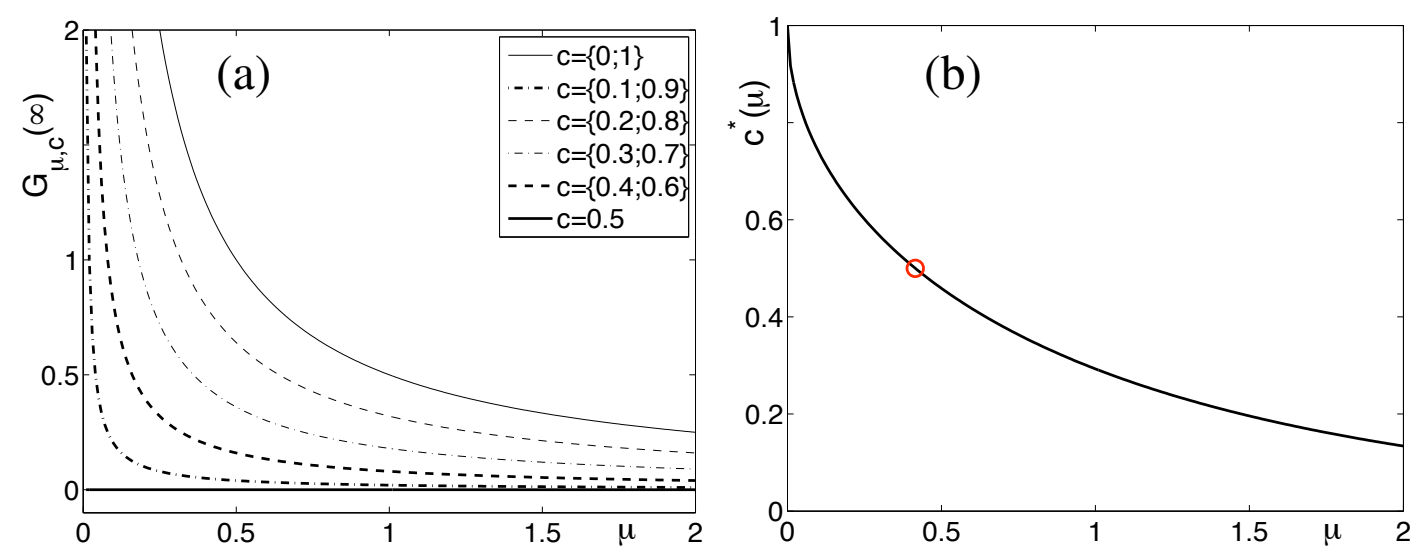

Figure 3.14: (a): Small scale asymptote of the factor $G_{\mu, c}(k)$ according to (3.20) as a function of $\mu$ for different source-target impact values $c$. (b): threshold value of the source-target impact $c^{\star}(\mu)$ according to (3.20), above which enhancement on scales $k^{-1} \sim \xi$ appears, as a function of Lévy exponent $\mu$.

small scales asymptotics from below. One of the messages from the picture is that we cannot obtain enhancement on all scales simultaneously. We can see, that for small $c$ (strong source impact), the function $G_{\mu, c}(k)$ is always positive, and the process is most attenuated (the global maximum) on the scales slightly above the correlation length. On scales approximately equal to the correlation length, the process attenuation is least pronounced (global minimum). Note that in the case $c=0$, for an intermediate Lévy exponent $(\mu=1)$, the long and small scales asymptotics coincide, suggesting the special role of this exponent for superdiffusion among traps: the only source dependent processes with $\mu=1$ exhibit the same dynamics except on the scales of the correlation length. For larger $c$ values, the global maximum becomes less pronounced. The whole long-scale range acquires similar relaxation properties and is attenuated. As previously mentioned, on the scales of the correlation length, the process becomes enhanced after $c^{\star}(\mu)$ (3.20). On smaller scales, enhancement disappears and $G_{\mu, c}$ attains its non-negative small scales asymptotics. For even larger values $c>c_{\text {crit }}$, the process is enhanced on large scales, the enhancement slows with decreasing spatial scales. Next in the case $\mu=1.5$ the enhancement gives place even to attenuation, then the enhancement increases again and after $k^{-1} \sim \xi$ diminishes towards zero. Afterwards the process becomes attenuated, reaching its most attenuation in the small scale limit. Note, that for the extreme $c$ values $(c=0$ and $c=1$ ), while small scale limits are the same, as was also the case in the diffusive limit, the only source-dependent process exhibits a faster rate of convergence to the asymptotic limit and thus more pronounced attenuation.

Thus, we can conclude that the strong acceleration regime at the scales of the correlation length may be attained also in the ordinary diffusive limit. Acceleration is mostly pronounced in the superdiffusive case of high target impact and cannot be realized at all scales. A largescale limit may be of particular interest, because the processes exhibits more universal properties in this case. In the next section, we discuss large scale asymptotics in detail, utilizing the results of Section 3.2. 


\subsection{Behavior on large scales}

In many physical situations, one is concerned with the asymptotic dynamics on large scales. If the inhomogeneity of the environment has an impact on large scales, it is certainly of importance and cannot be neglected. Moreover, it provides the theory with a scent of universality. As was already explained by the scaling reasoning (Section 3.1), this is exactly the case for the generalized topological superdiffusion. In what follows, we make use of the analytical perturbative results from the Section 3.2 for weak periodic and random inhomogeneities. In order to access their range of validity we also compare them to the numerical results.

\subsubsection{Periodic potentials}

The lowest band $E_{0}(q)$ with the small Bloch phase $q \ll 1$, and the corresponding wave number $k=q / \lambda \ll 1$, determines the asymptotic behavior on large scales. We characterize the dynamics on corresponding scales by the generalized diffusion coefficient. To this end we nee to calculate the limit

$$
D_{\mu, c}=\lim _{q \rightarrow 0} \frac{E_{0}(q)}{-(q / \lambda)^{\mu}} .
$$

According to (3.11), the generalized diffusion coefficient for weak potentials can be expressed as

$$
D_{\mu, c}(q ; \varepsilon) / D=1-\varepsilon^{2} G_{\mu, c}(q),
$$

with $G_{\mu, c}(q)$ given by (3.12). We can calculate the small- $q$ asymptotic by approximating the sum $2 \sum_{m>0} g_{\mu, c}(q / m)\left|\hat{v}_{m}\right|^{2}$ by the product of $g_{\mu, c}(q \rightarrow 0)$ and $2 \sum_{m>0}|\hat{v}|^{2}=1$. The last sum equals unity due to our choice of potential with unit variance and because $\int_{2 \pi \lambda} v^{2}(x) d x=$ $\sum_{m}\left|\widehat{v_{m}}\right|^{2}$ holds (Parseval or Plancherel theorem). The asymptotic limit of the function $g_{\mu, c}(z)$ given by (3.10) for small argument values $z \rightarrow 0$, corresponding to small Bloch phase values $q \rightarrow 0$ reads

$$
g_{\mu, c}(z)=1-2 c^{2}-\mu(1+\mu) c^{2} z^{2-\mu}+o\left(z^{2-\mu}\right)
$$

and thus

$$
\lim _{z \rightarrow 0} g_{\mu, c}(z)=\left\{\begin{array}{c}
1 / 2-c^{2}, 0<\mu<2, \\
1 / 2+2 c^{2}, \mu=2 .
\end{array}\right.
$$

Hence follows

$$
\lim _{q \rightarrow 0} G_{\mu, c}(q)=\left\{\begin{array}{c}
1 / 2-c^{2}, 0<\mu<2, \\
1 / 2+2 c^{2}, \mu=2 .
\end{array}\right.
$$

\subsubsection{Random potentials}

Recalling the results from Section 3.2 for random potentials and calculating the small- $k$ asymptotic of $G_{\mu, c}(k)=\frac{1}{2 \pi} \int d q S(q) g_{\mu, c}(k / q)$ given through (3.14) by factorizing it into $\frac{1}{2 \pi} \int d q S(q)=$ 1 and $g_{\mu, c}(z \rightarrow 0)$ given by (3.21) we obtain

$$
\lim _{k \rightarrow 0} G_{\mu, c}(k)=\left\{\begin{array}{c}
1 / 2-c^{2}, 0<\mu<2 \\
1 / 2+2 c^{2}, \mu=2
\end{array}\right.
$$




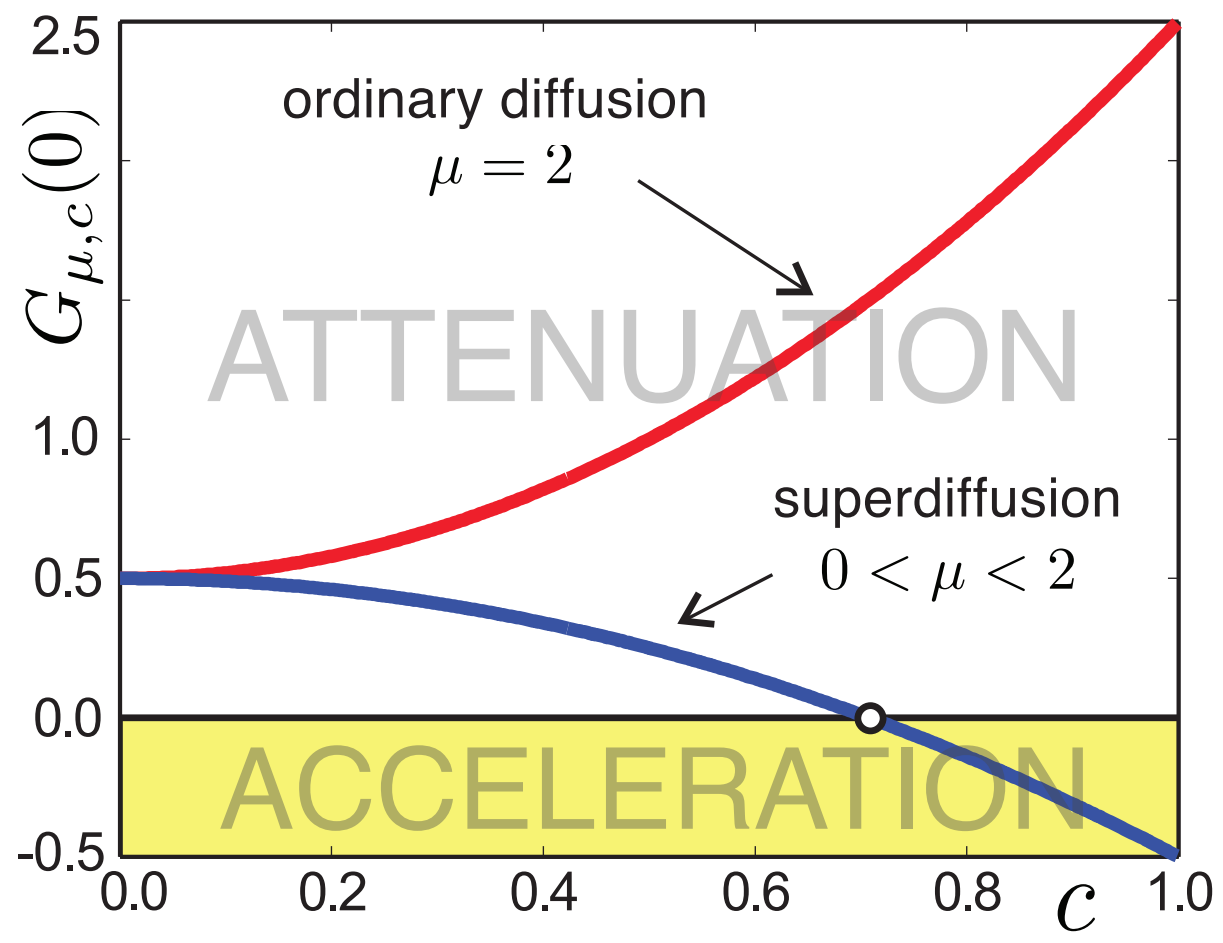

Figure 3.15: The impact of source and target location on the asymptotic of random walk processes. The generalized diffusion coefficient depends quadratically on the magnitude $\varepsilon$ of the inhomogeneity: $D_{\mu, c} / D=1-\varepsilon^{2} G_{\mu, c}(0)$. The factor $G_{\mu, c}(0)$ quantifies the impact of the spatial inhomogeneity, i.e. when $G_{\mu, c}(0)>0$, the process is slowed down, when $G_{\mu, c}(0)<0$, the process is accelerated. Two lines depict $G_{\mu, c}(0)$ as a function of the weight parameter $c$ for ordinary diffusion ( $\mu=2$, upper line) and the Lévy flights $(0<\mu<2$, lower line). As $\mathrm{c}$ is increased (increasing weight of the target location), ordinary diffusion is slowed down more strongly. This stays in contrast to the Lévy flights for which attenuation decreases until a critical value of $c_{\text {crit }}=1 / \sqrt{2}$ is reached (denoted by a small circle). Above this source-target impact, the Lévy flights are accelerated by the external inhomogeneity.

which coincide with the expression (3.22) for periodic potentials. This evidences the universal behaviour of source- and target-dependent processes in weak inhomogeneities.

The factor $G_{\mu, c}(z)$ as a function of $\mu$, does not converge uniformly on the interval $\mu \in(0,2]$. Ordinary diffusion limit $(\mu=2)$ presents an exceptional case, though we have a universal behavior for all superdiffusive exponents $\mu \in(0,2)$. The counter-intuitive independence of the generalized diffusion coefficient as a function of $\mu<2$ from its argument, is attained only in an infinite system. In Section 3.6, we will see that there is no such an abrupt transition from superdiffusive to ordinary diffusive exponents in the case of finite systems. For a discussion, see also (Brockmann, 2003; Brockmann and Geisel, 2003b). The discontinuity in the response to an external potential field is present only in the large scale asymptotic and for weak potentials.

The dependence of the asymptotics factor $G_{\mu, c}(0)$ on the source-target impact factor $c$ for ordinary diffusive as well as superdiffusive processes is presented in Figure 3.15. Typically, a random spatial inhomogeneity slows down a random process, and one generally expects 
$D_{\mu, c}(0 ; \varepsilon)$ to be smaller than $D_{\mu, c}(0 ; \varepsilon=0)$. As one can see from Figure 3.15, with increasing influence of the target salience (increasing values of $c$ ), superdiffusive processes $(\mu<2)$ exhibit the opposite behavior as ordinary diffusion processes $(\mu=2)$. Only when $c=0$, and target salience has no impact on the transition rate, all processes are slowed down by the same amount, i.e. $G_{\mu, 0}(0)=1 / 2$. As $c$ is increased, $G_{\mu, c}(0)$ increases as well for ordinary diffusion indicating that these processes are slowed down more strongly. This stands in contrast to superdiffusive processes, for which a more pronounced target influence decreases $G_{\mu, c}(0)$. This implies that attenuation of superdiffusive processes becomes weaker as the target weight in the transfer rate is increased. Moreover, if the impact of the target location ( $c$ exponent) is high enough, we obtain enhancement of the superdiffusion ${ }^{5}$. Equation (3.21) implies that the critical impact is $c_{\text {crit }}=1 / \sqrt{2} \approx 0.7071$. It is also a non-trivial result which claims that the impact of the target is more important than the impact of the source location. For acceleration to appear the impact of the target does not only need to becomes larger than the source impact, it needs to increase beyond the critical value. This is an interesting and novel result(Belik and Brockmann, 2007). Consequently, the common notion that random processes are typically slowed down by spatial inhomogeneities is not valid when superdiffusive processes are involved.

\subsection{Numerical results}

For small and intermediate values of the inhomogeneity strength $\varepsilon$, we computed the generalized diffusion coefficient $D_{\mu, c}(\varepsilon)$, characterizing the dynamics on large scales numerically for four periodic (A-D) and three random phase potentials (E-G) introduced in Section 3.1. Figures 3.16 and 3.17 present the dependence of the generalized diffusion coefficient on the potential strength $\varepsilon$.

The most amazing feature is a qualitative difference between ordinary diffusion and Lévy flights for large values of the source-target impact predicted by the theory. In this regime, ordinary diffusion processes are still slowed down by the spatial inhomogeneity as opposed to the Lévy flights, which exhibit an increase in the diffusion coefficient with increasing potential strength and are thus accelerated. Indeed, for $c=3 / 4$ and $c=1$ we have a profound increase of the diffusion coefficient with the potential strength $\varepsilon$. The value of the coefficient is above unity, implying enhancement of the process as compared with the homogeneous case. For $c=0$ (i.e. full weight of a source location), processes in a given environment behave similarly independent of the exponent $\mu$. Ordinary diffusion and all superdiffusive processes exhibit the same quadratic decrease of $D_{\mu, c}$ with $\varepsilon$ in a fixed potential. For $c=1 / 2$, the impact of the potential shape on the ordinary diffusion is the least and is increasing on both sides of $c=1 / 2$, though it is most pronounced for the solely source-dependent processes. This fact contradicts the statement that ordinary diffusion is not affected by the inhomogeneity shapein the balanced case (Brockmann, 2003). Note that superdiffusive processes show the least variability for the source-target impact in the vicinity of $c_{\text {crit. }}$. The curves collapse on one single function. At this

${ }^{5}$ Because $G_{\mu, c}(0)$ for superdiffusive processes becomes negative implying that these processes are accelerated by the spatial inhomogeneity since a negative value for $G_{\mu, c}(0)$ implies the diffusion coefficient larger than in the case of free superdiffusion. 

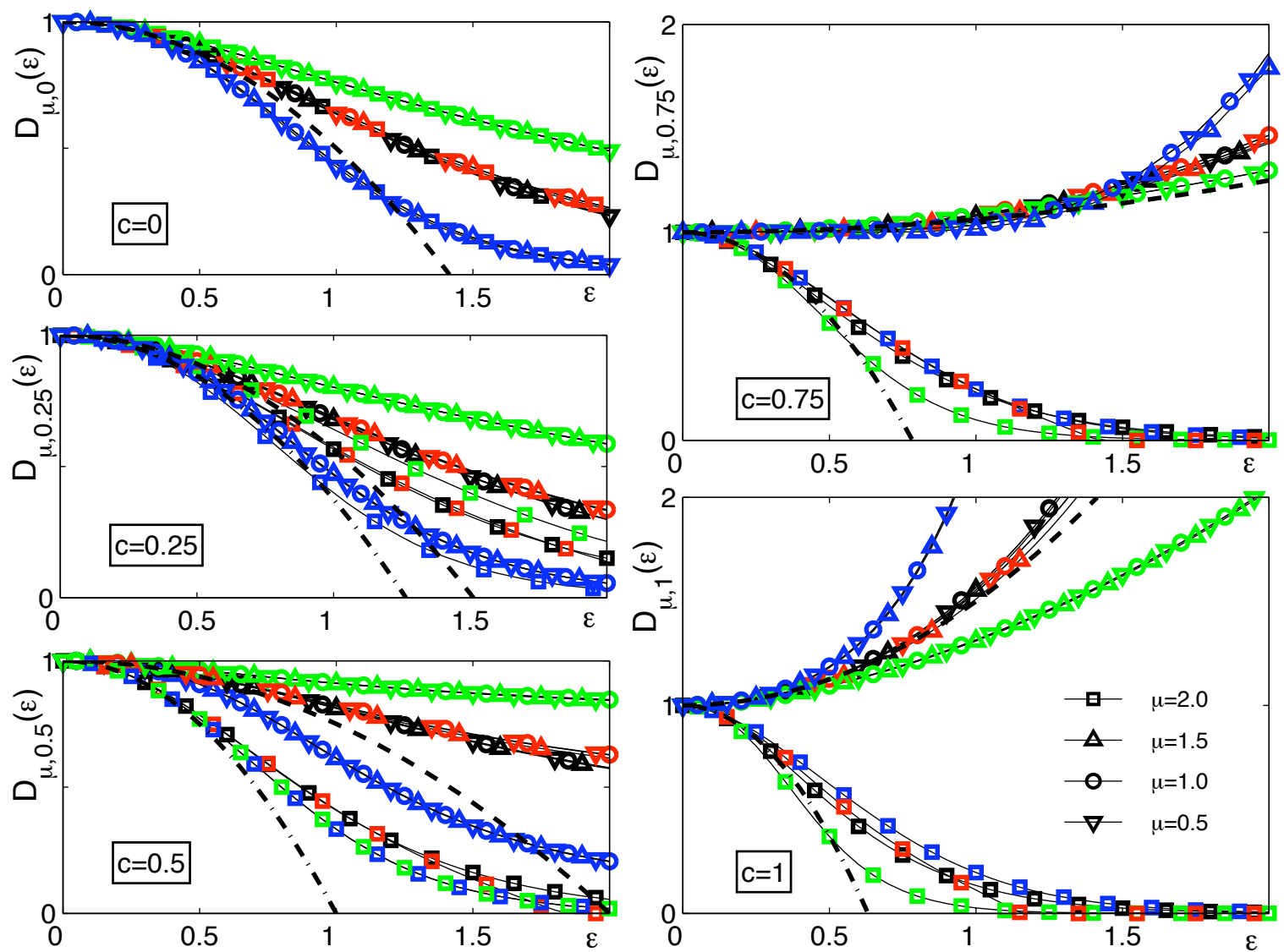

Figure 3.16: Generalized diffusion coefficient $D_{\mu, c}$ as a function of the magnitude $\varepsilon$ of the salience field $s(x)=\exp [-\varepsilon v(x)]$. Diffusion coefficient of the homogeneous system is set to unity. The function $D_{\mu, c}$ is shown here for four types of periodic potentials: a cosine potential (A, black color), piecewise potential (B, red color) and potentials with localized maxima and minima, $(\mathbf{C}$ and $\mathbf{D}$, green and blue colors, respectively). Potentials are displayed in Figure 3.2. Results for five values of the weight parameter $c$, are presented: $c=0 ; 0.25 ; 0.5 ; 0.75$ and 1 . Symbols and solid lines denote the numerical results. The dashed line and the dash-dotted lines are analytical results for $\mu=2$ and $0<\mu<2$ obtained by perturbation theory (3.22). Each type of symbol corresponds to one Lévy exponent: $\circ-\mu=0.5, \diamond-\mu=1.0, \triangle-\mu=1.5, \square-\mu=2.0$. For $c=1$ in contrast to diffusive processes, which are attenuated, the superdiffusive ones are enhanced by inhomogeneity. In Figure 3.17 results for random potentials are presented.

point, inhomogeneities with opposite bias swap their position. If for $c=0$, processes in the wells potential were the most attenuated, for $c=1$, they are the most accelerated. If for $c=0$, processes in the peaks potential were the least attenuated, for $c=1$, they are the least accelerated. This again emphasizes the strongest influence of deep attractive wells on superdiffusive processes.

Based on the concept of salience, we can provide an intuitive explanation of the acceleration of the superdiffusive walker for $c>1 / \sqrt{2}$. If the salience of the target site grows, than due to the non-locality of the Lévy flights, the random walker moves effectively only among 
regions of attraction with highest salience. This leads to the enhanced exploration and thus to acceleration of the process. On the contrary, diffusive walkers are only aware of their immediate surrounding and are trapped in confined regions.
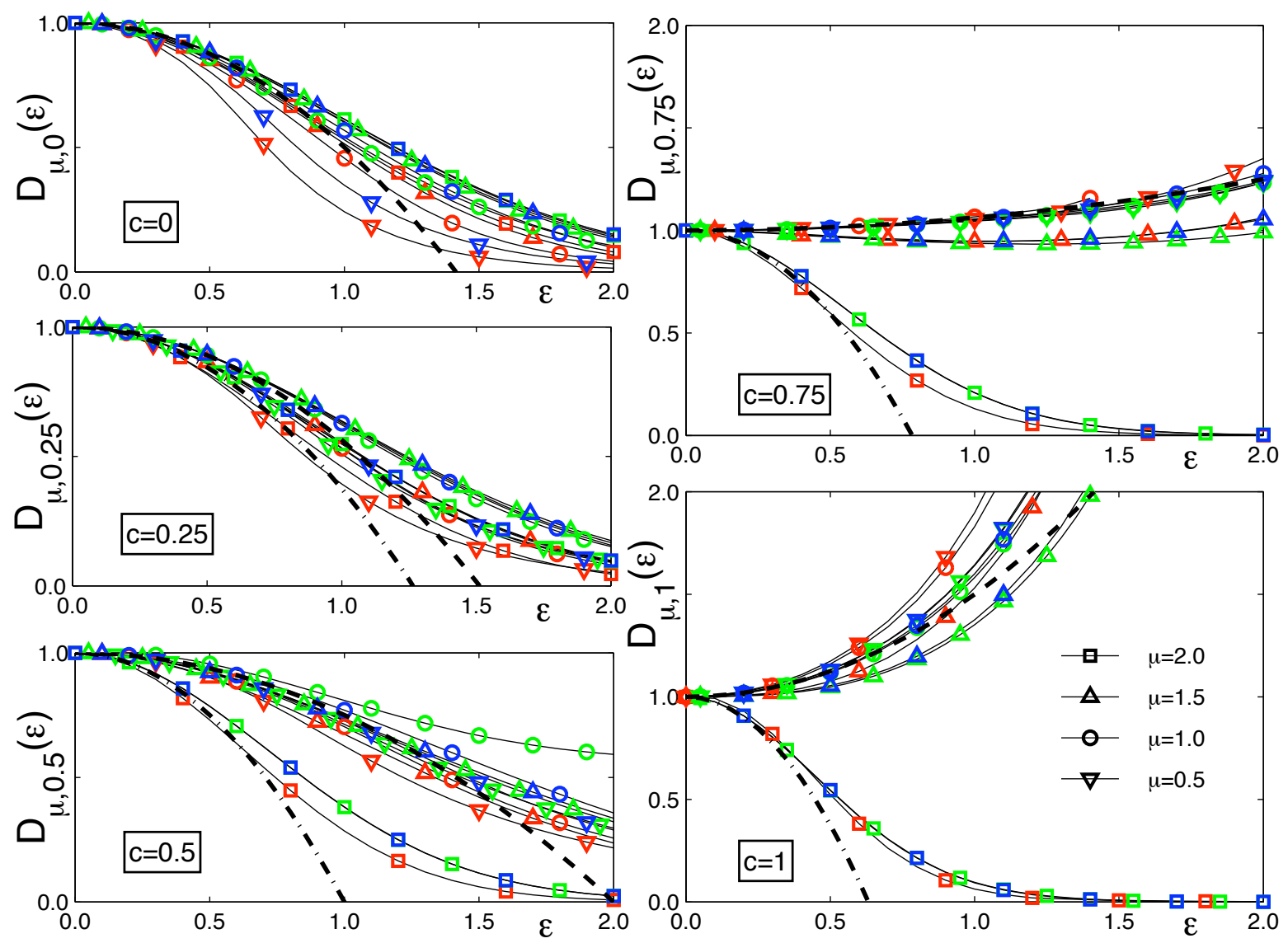

Figure 3.17: Generalized diffusion coefficient $D_{\mu, c}(\varepsilon)$ in dependence on the inhomogeneity strength $\varepsilon$ for different source-target impacts $c$ for three random potentials with Heaviside (E, red color), Gaussian (F, green color) and exponential power spectrum ( $\mathbf{G}$, blue color). Diffusion coefficient in the homogeneous system is set to unity. For other details see Figure 3.16, where results for periodic potentials are presented.

Results for random potentials presented in Figure 3.17 agree very well with results for periodic potentials. One can observe that variability in respect to different Lévy exponents increases with a roughness of the random potentials, which in turn increases with the correlation length. Random potentials were chosen in a way that the correlation length increases from $\mathbf{E}$ to $\mathbf{G}$ (Figure 3.2).

We have considered the consequences of the relative weight of source and target locations in one-dimensional random walk processes, evolving in an inhomogeneous environment. Our analysis revealed essential differences between superdiffusive Lévy flights and ordinary random walks when they occur in regular and random spatial inhomogeneities. Both superdiffusive and ordinary diffusive processes can distinguish among inhomogeneities of different 
shapes. For small values of $\varepsilon$, the numerics agree well with our results obtained by the perturbation theory. Unlike ordinary random walks, Lévy flights can be accelerated on large scales when the influence of the target salience is sufficiently large.

\subsection{Finite systems}

The considerations presented in previous sections were restricted solely to the infinite systems. A key question raised now is how these processes behave in finite systems, and to what extent finite size effects play a role. We can easily access finite size effect in periodic inhomogeneities. Therefore, we investigate the relaxation properties in finite systems of length $2 \pi L$, modulated by the periodic potential of the wavelength $2 \pi \lambda$. Let us consider the quantity

$$
\delta \tau=\tau / \tau_{\text {free }}-1
$$

where $\tau$ is the relaxation time of the process and $\tau_{\text {free }}$ is the relaxation time of the same process without spatial inhomogeneity. In a finite system, the corresponding Hamiltonian has a discrete spectrum. The continuous Bloch bands (for the infinite system) split into $M=L / \lambda$ discrete eigenvalues $E_{n}\left(q_{m}\right), n=0 . . \infty, m=1 . . M$, where $q_{m}=m \lambda / L$ is a Bloch phase. The smallest Bloch phase is given by $q_{1}=\lambda / L$. The relaxation time is related to the smallest eigenvalue of the spectrum by $\tau=1 / E_{0}(\lambda / L)$. Considering (3.24) and recalling that $\tau_{\text {free }}=L^{\mu}$, we obtain from (3.12)

$$
\delta \tau \approx \varepsilon^{2} G_{\mu, c}(\lambda / L)=\varepsilon^{2} 2 \sum_{m>0} g_{\mu, c}(m \lambda / L)\left|\hat{v}_{m}\right|^{2}
$$

which reads most simply: $\delta \tau \sim \varepsilon^{2} g_{\mu, c}(\lambda / L)$ for the cosine potential because all its Fourier coefficients vanish except the first one. In Figure 3.18, the dependence of the factor $G_{\mu, c}(\lambda / L)$ on the Lévy exponent $\mu$ is depicted for five values of $c=0,1 / 4,1 / 2,3 / 4$ and 1 and different $\lambda / L$ ratios. Small values of $\delta \tau$ correspond to a small effect of the inhomogeneity. For $c=0$, the ordinary diffusion process exhibits the smallest value of $\delta \tau$, which implies that in situations in which the source salience is important, diffusion exhibits the fastest relaxation. On the contrary, for $c=1$, strongly superdiffusive processes (i.e. $\mu \rightarrow 0$ ) exhibit the smallest $\delta \tau$. Only when both source and target possess an equal impact on the jump rates $(c=1 / 2), \delta \tau$ exhibits a minimum for intermediate values of the Lévy exponent $\mu$ (Brockmann and Geisel, 2003a). See also the discussion in the next paragraph on the optimal Lévy exponent. Intermediate $c$ values $c=1 / 4$ and $3 / 4$ shows transitions between the above-mentioned regimes. In finite systems, there is a continuous transition from attenuation to acceleration with varying exponent $\mu$. In Figure $3.18 c=1$ one can see, that only the Lévy flights with small exponents can be accelerated.

In small systems, one can see some dependence on the kind of potential. However, differences disappear in larger systems. One observes that the convergence of $G_{\mu, c}(\lambda / L)$ to its asymptotic value depends strongly on the parameter $c$. One can also note a similarity in the convergence in potentials with seldom peaks and seldom wells. Processes in the cosine and piecewise constant potential share strong similarities too. If one changes the parameter $c$ from 

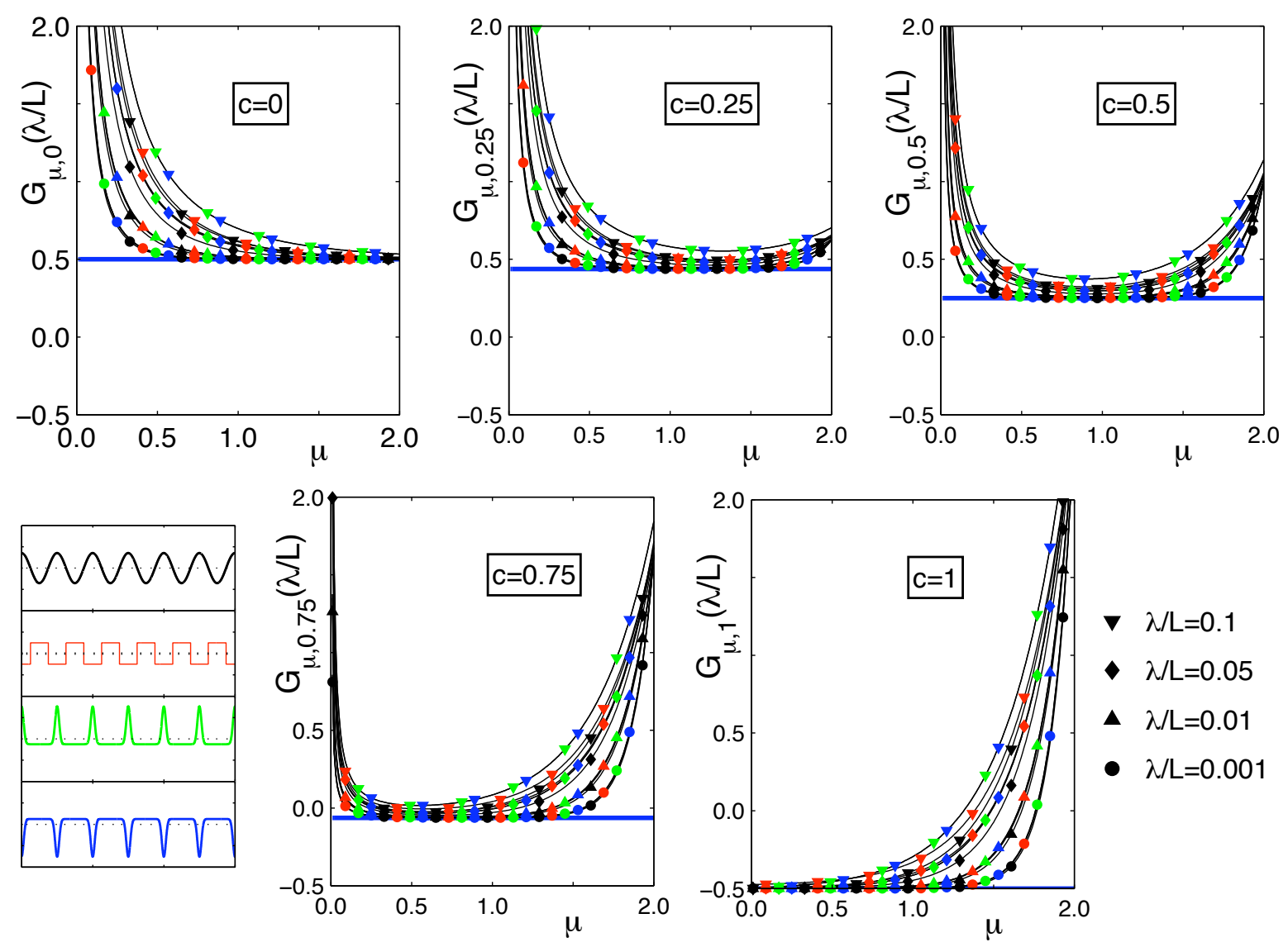

Figure 3.18: The impact of the inhomogeneity on the relaxation time of the processes $\delta t \sim G_{\mu, c}(\lambda / L)$ in dependence on the size of the system $2 \pi L$ for the different periodic potentials (denoted by color code on the inset) with period $2 \pi \lambda$. Different power-law exponents $\mu$ and different values of the weight parameter $c$ were considered. Blue lines denote the large scale limit, according to (3.22). With increasing $L / \lambda$ ratio for $c=1$, acceleration corresponding to negative values of $G_{\mu, c}$ occurs for increasing range of large $\mu$ exponents.

zero to unity, from only source-dependent processes to only target-dependent processes, one observes different speed of convergence towards infinite system behavior for different Lévy indexes $\mu$. For only source-dependent processes $(c=0)$, the fastest convergence occurs in the ordinary diffusive case $\mu=2$. For intermediate $c$ values, processes with $\mu \approx 1$ outperform ordinary diffusion and strongly superdiffusive processes. This feature for $c=1 / 2$ was used as a supporting argument for the explanation of the fact that foraging albatrosses perform Lévy flights with $\mu \approx 1$ (Viswanathan et al., 1999a,b), cf. also debate in Reference (Edwards et al., 2008). In the case $c=1 / 2$, this exponent leads to fast relaxation in inhomogeneous environment. However, as it can be seen from Figure 3.18, it is not the only option if we allow unequal source and target contribution. The optimal foraging coefficient is thus determined by the $c$ value. For large $c$, strongly superdiffusive processes relax in the fastest way. With changing $c$, optimal $\mu$ goes from ordinary diffusive value $\mu=2$ to strongly superdiffusive values $\mu \rightarrow 0$. The alleged optimality of the Lévy exponent $\mu=1$ (Viswanathan et al., 1999b) occurs only in 
the case when source and target are contribute equally to the transition probability. Thus, the optimal foraging index $\mu$ is not restricted to approximately unity. To determine it, we should also take into account the impact on the process of temporary source and target.

\subsection{Global coupling}

We now want to discuss a modification of our model, based on the gravity law assumption. Reminiscent of the dynamical game theory (Nowak, 2006) we consider a normalized salience implying the global coupling, i.e. instead of $s(x)=e^{-\varepsilon v(x)}$, we consider $s(x) /\langle s\rangle$, where averaging is performed over the whole available support. We can then carry out perturbation analysis in $\varepsilon$ similar to the case of non-normalized salience (see Appendix). We obtain in the large-scale limit for the factor $G_{\mu, c}$ (cf. (3.23) ):

$$
\lim _{k \rightarrow 0} G_{\mu, c}(k)= \begin{cases}-c(c-1) & 0<\mu<2, \\ c+2 c^{2} & \mu=2 .\end{cases}
$$

The dependence of $G_{\mu, c}(0)$ on the source-target impact $c$ is presented in Figure 3.19. As one can observe, contrary to the case of non-normalized salience in the superdiffusive case, acceleration is absent. Source and target influence superdiffusive processes in a symmetric way. Only source-dependent processes in both ordinary diffusive and Lévy flight case do not feel weak inhomogeneity. With increasing $c$, both ordinary diffusive and superdiffusive processes are attenuated, but to different extents. Superdiffusion is less slowed down by the inhomogeneity. After reaching the balanced case $(c=1 / 2)$, paths of ordinary diffusion and Lévy flights split. While attenuation of the ordinary diffusion still increases with $c$, Lévy flights become less attenuated. At $c=1$, i.e. in the target-dependent case the superdiffusion, again as for $c=0$, does not feel inhomogeneity at all. In the case of ordinary diffusion, attenuation is maximal at $c=1$. For a comparison, the dependence of $G_{\mu, c}(0)$ on $c$ with a non-normalized salience is also presented in Figure 3.19.

These results indicates that as far as the global information becomes available and poles of attraction become relative, the most Lévy flights can gain is a vanishing of the inhomogeneity. For this purpose one needs to choose source- or target-dependent travel rates. There is no way for acceleration in such a system.

\subsection{Perspectives}

So far, we considered consequences of the gravity model assumption for exclusively diffusive or superdiffusive transport of individuals in inhomogeneous environments. Bearing in mind human or animal populations (with usually many different species) we need to account for interactions between individuals. We can choose among many different possible interaction dynamics.

For example, we can consider an autocatalytic reaction mechanism $I+S \rightarrow 2 I$ describing a susceptible-infected epidemics (cf. Chapter 2). For such kinds of reaction-diffusion systems 


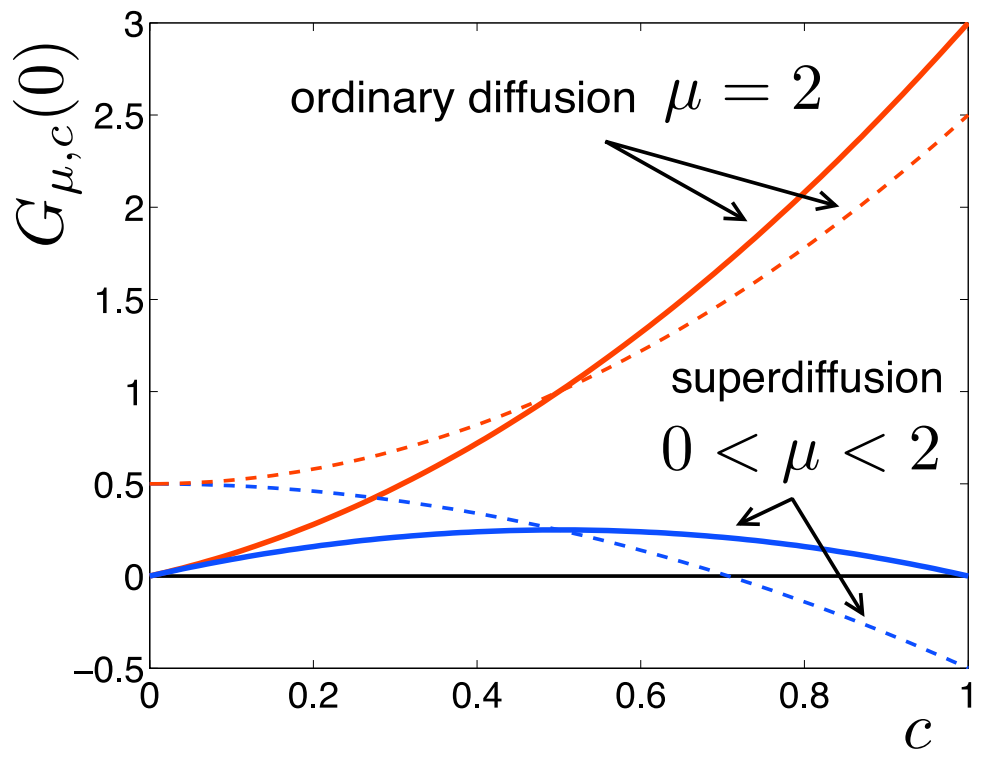

Figure 3.19: Dependence of the factor $G_{\mu, c}(0)$ on the source-target impact $c$ for normalized (solid color lines) and non-normalized (dashed lines) salience. There is no acceleration of superdiffusive processes in the case of normalized salience.

powerful field-theoretical tools exist (Doi, 1976b,a; Kree et al., 1989; Lee, 1994). We do not proceed in this direction, but note that one attempt to investigate the role of the inhomogeneity in the balance case of topological superdiffusion $(c=1 / 2)$, and for annihilation reaction of the type $A+A \rightarrow 0$ was already undertaken in (Chen and Deem, 2003). A starting point of their investigations was an equation for the Green's function $G(x, t)$, describing superdiffusion which can be derived by linearization in weak inhomogeneities. We derived a similar equation in the general case $c \in[0,1]$, which reads

$$
\partial_{t} \hat{G}(k, t)=-r_{0}^{\mu} k^{\mu} \hat{G}(k)+\varepsilon r_{0} \int d k^{\prime} \hat{V}\left(k-k^{\prime}\right) \hat{G}\left(k^{\prime}\right)\left\{c k^{\mu}+(c-1)\left[\left(k^{\prime}\right)^{\mu}-\left|k-k^{\prime}\right|^{\mu}\right]\right\}
$$

where $r_{0}$ is the minimal possible space distance or cutoff and $V$ is the potential defining the salience. One can try to follow a similar way of reasoning to reveal an impact of disbalanced source and target influence on the reaction dynamics of agents performing Lévy flights in inhomogeneous environments.

Another possible reaction mechanism pertinent to spatially extended Lotka-Volterra (Murray, 1993) systems manifests itself in the most simple form as the search for resources. Search processes play an important role in many fields from animal foraging (Viswanathan et al., 1999a; Bénichou et al., 2005; Edwards et al., 2008) and to routing protocols in the Internet (Toroczkai et al., 2008). They are also closely connected to the first passage problems (Feller, 1971; Condamin et al., 2008). Recalling findings of Section 3.6, we may state that better relaxation and therefore better exploration, occurs not only for $\mu=1$ (Raposo et al., 2003), but for different Lévy exponents dependent on the source-target impact. If the target impact prevails then the most optimal strategy would be the superdiffusive strategy. If the source influence is strong ordinary diffusion would be the best choice. Moreover, different modifications are imaginable such as a source-target impact depending on the internal, e.g sated or hungry state of a forager. 
Another often neglected issue is an exaggeration of random walk models concerns immediate jump after some waiting times with infinite velocities. In this case the spatial and temporal increments are decoupled from each other. As some recent studies suggest (Zaburdaev et al., 2008), if spatial and time increments are taken into account, essential differences in the evolution of the propagator of such a process appear. One can imagine that similar effects may appear also in the context of diffusion in disordered environments. Moreover, one can think of dependence of velocity increments on source and destination locations. Such kinds of problems are of importance for optimal random search strategies.

One of the exotic questions initially posed by Feynman (Feynman et al., 1966) can be raised with respect to the topological superdiffusion as well. It is an investigation of motion in periodic potentials lacking symmetry. In such a system for ordinary diffusion one can obtains rectification, i.e. persistent current in one direction, the so-called ratchet (Astumian and Bier, 1994). In Dybiec et al. (2008), an attempt to consider a ratchet with Lévy flights was undertaken, in which the usual generalized Langevin approach to superdiffusion was used. It may be interesting to consider the effects of source-target impact in such a system.

\subsection{Summary and discussions}

In the present chapter, motivated by the possible application in the population dynamics and epidemiology we have introduced a model for a random walk in an inhomogeneous environment. In the model, the transition rate of the random walk depends on both temporary source (or start) and on temporary target (or destination) locations. This dependence resembles the gravity law assumption having a long merited history of application in social and economical sciences (Zipf, 1946; Xia et al., 2004). The gravity assumption states that a flow of goods or individuals between a pair of locations is proportional to the population of both of them raised to a $c$-th power and is weighted by some negative power of the distance between them. The exponent of the distance determines whether we have ordinary diffusion or Lévy flights. Despite the fact that this model is only an approximation of reality, it is long-standing and has proven to be a good null hypothesis accounting for human movements. Besides, one can view the proposed model as a generalization of the topological superdiffusion (Brockmann and Sokolov, 2002; Chen and Deem, 2003) which can be recovered from our model in the case of balanced source-target impact corresponding to $c=1 / 2$. In the course of our analysis, different kinds of inhomogeneities — periodic and random ones — were considered. We have used perturbative methods to establish the theory of the generalized topological superdiffusion in weak inhomogeneities (Section 3.2), paying particular attention to dynamics on large scales (Section 3.4). In order to investigate the dynamics on intermediate scales, we have used the Bloch theory for periodic inhomogeneities and analyzed the spectral properties obtained by the perturbation theory in the case of random inhomogeneities (Section 3.3). We compared our theoretical predictions with numerical results in Section 3.4 as well as explored finite size effects in Section 3.6.

Our results revealed a striking difference in behaviour of ordinary diffusion and superdiffusion in inhomogeneous environments with a varying source-target impact. We have shown that superdiffusive motion can lead to enhancement of dispersion with a growing disorder 
strength. This is a rather counterintuitive result, because disorder usually attenuates dispersion. While with increasing target impact we obtain an increasing attenuation of the dispersion in the case of ordinary diffusion, we observe a diminished attenuation and even enhancement of the process above the critical source-target impact in the case of superdiffusion. For a superdiffusive process to be accelerated, it is not sufficient for the target just to prevail over the source influence but the impact of the target ought to be strong enough. To be specific, the source-target influence measured by the parameter $c$ has to be greater then $c_{\text {crit }}=1 / \sqrt{2}$. Our results state that, as far as random walkers move superdiffusively between inhomogeneously populated locations and aim to attain the fastest dispersion, an optimal source-target impact parameter should be equal to unity, what is also near an empirically found value for humans (Xia et al., 2004). Based on the concept of salience or attractiveness of a location, we can provide a heuristic explanation as to the acceleration of a superdiffusive walker. If the salience of the target site grows, then due to the non-locality of the Lévy flights, the random walker moves effectively only among regions of attraction with highest salience, which leads to enhanced exploration and thus to acceleration of the process. On the contrary, diffusive walkers are only aware of their immediate surrounding and are trapped in confined regions.

However, the analysis of the dynamics on intermediate scales showed that the enhancement cannot be obtained simultaneously on all scales. While dispersion on large scales experiences acceleration, dynamics on intermediate and small scales exhibits attenuation. What concerns the influence of the inhomogeneity on intermediate and small scales is that there are some differences in sensitivity between ordinary diffusive and superdiffusive processes. Although for ordinary diffusion, the sensitivity with growing $c$ becomes smaller (but still substantial), the above mentioned difference almost disappears for Lévy flights at $c \approx c_{\text {crit }}$ and for greater values of $c$ becomes pronounced again. On small scales, the case of balanced source-target impact exhibits the least attenuation. The most pronounced difference among distinct inhomogeneities occurs for the strongly source dependent processes for both ordinary diffusive and superdiffusive processes. Compared with all examined inhomogeneities, an environment with seldom regions of high attraction affects superdiffusive processes to the greatest extent. Contrary to the statement that ordinary diffusion is not affected by a particular shape of an inhomogeneity (Brockmann, 2003), in the disbalanced case $c \neq 1 / 2$, even ordinary diffusion can very well distinguish between inhomogeneities with an opposite bias (Section 3.3). The effect of acceleration on the scales of correlation length for even ordinary diffusion is most pronounced for processes with a high target impact.

In the large scale limit, a responce to inhomogeneity in infinite systems exhibits a discontinuity at $\mu=2$. This disappears in the case of finite systems. Thus, acceleration in such systems can only be achieved for a sufficiently small Lévy exponent. Convergence to the large scale limit results is different for different Lévy exponents. This leads to generally different optimal Lévy exponents given a fixed $c$. Hence a believed optimality of intermediate $\mu \approx 1$ exponents (Viswanathan et al., 1999a) is not universal. For small $c$ values, the most optimal exponent corresponds to ordinary diffusion and for large $c$ values it is a strong superdiffusive limit $\mu \rightarrow 0$. The exponent $\mu \approx 1$ becomes optimal only if the source and target influence is balanced.

If global information on salience is available everywhere, we cannot achieve acceleration anymore even in the superdiffusive case (Section 3.7). While ordinary diffusion keeps its 
qualitative behavior from the non-normalized case, Lévy flights, instead of decreasing with $c$, experience a symmetric influence of the source and target locations and exhibit the maximal attenuation at the balanced case. These results indicate that, as far as the global information become accessible and poles of attraction become relative, the most what Lévy flights can gain is an effective vanishing of the inhomogeneity. For this purpose, one needs to choose sourceor target-dependent travel rate. There is no way for acceleration in that system.

We briefly mentioned directions of further research in Section 3.8. In addition, we have discussed how to incorporate reaction in our model, what is of particular importance for epidemiological and ecological applications. In this respect, the use of field-theoretical tools can be helpful. Moreover, our results are of interest in the field of optimal random search algorithms in inhomogeneous landscapes (Condamin et al., 2008) and for a such exotic field as superdiffusive ratchets Dybiec et al. (2008). We believe that our findings may shed new light on many dispersal phenomena in population dynamical systems, various physical and biological contexts. 


\section{Epidemic spread due to recurrent host movements}

In Chapter 3, we have examined the role of disorder in a system, where agents perform superdiffusive random movements in inhomogeneous environment with transition rates dependent on the start and the destination location of a single movement event. We were mostly motivated by epidemiological applications, because human-mediated transport on large scales displays similar patterns (Brockmann et al., 2006). Infectious diseases can spread among different spatially distributed populations by the contacts between hosts from different locations. This happens due to host movements. As it was already mentioned in Chapter 2 (Fundamentals), there are two main frameworks aiming to incorporate movements of hosts into the description of an epidemic spread. The reaction-diffusion approach assumes that indistinguishable hosts can perform random walk among different habitats, i.e. diffuse. The second framework is referred to as the direct coupling approach. It suggests that local infectious dynamics are heuristically coupled to dynamics in other populations. Motivated by recent empirical studies (Brockmann et al., 2006; González et al., 2008) revealing recurrent and confined nature of human movements, in the present chapter we derive and analyse a novel general model for the epidemic spread explicitly incorporating recurrent host movements patterns. Both the reaction-diffusion and the direct coupling models represent special cases of our model. We show that dynamics of epidemics induced by recurrent host movements contrasts dramatically with the reaction-diffusion framework in many aspects, in particular, concerning the dependence of the velocity of spread on the individual travel rate.

The present chapter is structured as follows. In Section 4.1 we introduce our model and compare it with the directly coupled model in Section 4.2. In Section 4.3 we investigate an exemplary case of two locations. We proceed further by deriving the continuum version of our model in Section 4.4, analysing it analytically and numerically (Section 4.5) and compare it with the reaction-diffusion case and the continuum counterpart of the direct coupling model (Section 4.4.7). In Section 4.5 we analyze the results of the extensive agent-based stochastic computer simulations of our model, and analytically explain the deviations from the meanfield behavior. We sketch the models of epidemics due to recurrent movements on inhomogeneously populated random networks in Section 4.6. Eventually, we outline the directions of further research in Section 4.7.

\subsection{General model}

We consider a metapopulation (Hanski, 1998) inhabited by individuals distinguishable according to their locations of origin and moving with the origin-dependent transition rates. 


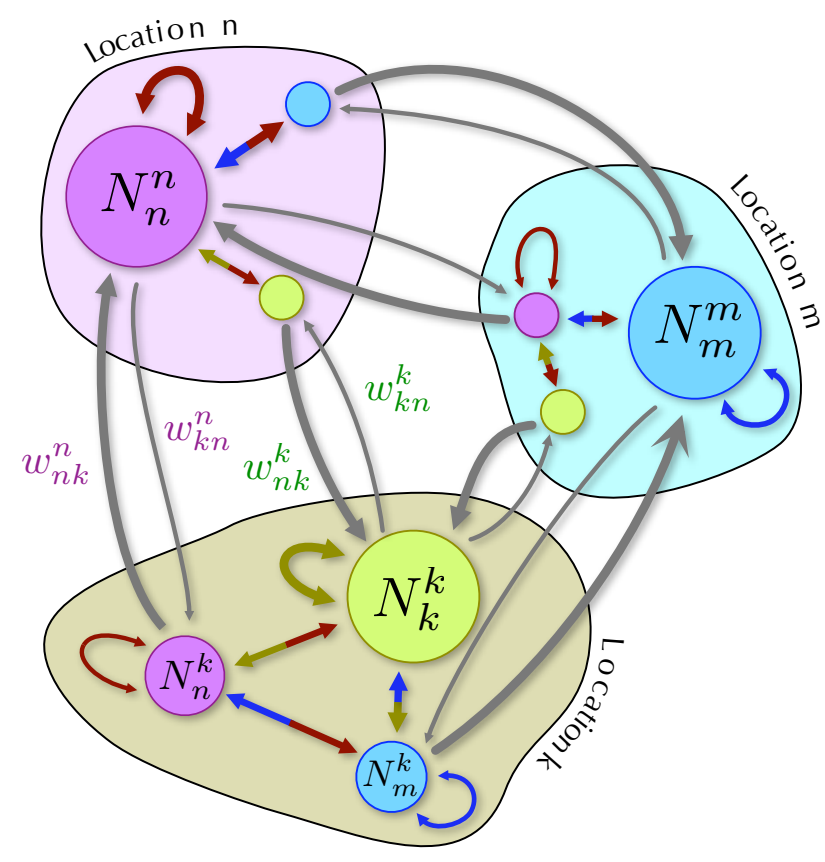

Figure 4.1: Schematic representation of the metapopulation model with travelling hosts. Within one location there can be species from other locations, which are denoted by circles. Different colors corresponds to species from different locations. The grey one-ways arrows symbolize movements among different locations. Two-way arrows symbolize interactions (infectious contacts) among different species. $N_{m}^{n}$ denotes a number of infected people from the $m$-th location sojourning in the $n$ th city. $\omega_{n k}^{n}$ denotes the travel rate of individuals with the $n$-th location as home (upper index) from the $k$-th location into the $n$-th location.

Furthermore, we impose that individuals do not remember where they were before and thus an underlying process is a Markov one (Gardiner, 1997). That humans forget their previous trips is an exaggeration, but it is more reasonable than the assumption of unbounded diffusion for their movement pattern. In the present section, we build an epidemic model with hosts moving with the origin-dependent transition rates.

Imagine a metapopulation distributed over $M$ locations, connected in some way to each other. Each individual is supposed to live permanently in one particular location. At every instant, an individual belonging to the $n$-th location can sojourn in some other location $m$. Thus, generally in every location and at every instant, individuals from other locations may be found (see Figure 4.1). We denote by $N_{n}^{m}$ the number of individuals belonging to the $n$-th location (indicated by the bottom index) sojourning in $m$-th location (indicated by the upper index). Individuals originating from the location $n$ may leave their current location $k$ and travel to some other location $m$ with the forward rate $\omega_{m k}^{n}$ and also return with the backward rate $\omega_{k m}^{n}$. By definition, movements from the location $n$ to the location $n$ are not considered and thus $\omega_{k k}^{n}=0$ for every $n$ and $k$. We assume that transition rates depend on the origin, which we denote by the upper index in the transition rates. In general, travel rates are given by the tensor quantities $\omega_{m n}^{k}$ where all indices can be independent from each other. This situation is equivalent to permitting the random walk with home-dependent transition rates. Such movement patterns can be formally represented by the following reaction scheme $\forall n, m, k$ :

$$
N_{n}^{k} \underset{\omega_{k m}^{n}}{\stackrel{\omega_{m k}^{n}}{n}} N_{n}^{m}
$$

If we discard fluctuations, we can write down the following multiparticle (chemical) master equations (van Kampen, 2007) for the overall probability density $\mathcal{P}\left(\ldots, N_{n}^{m}, N_{n}^{k}, \ldots\right)$ to find 


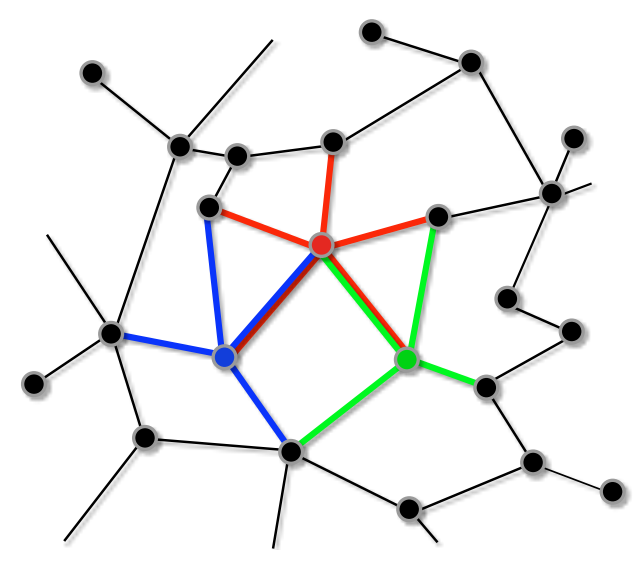

Figure 4.2: Schematic representation of recurrent host movements between their abode and other locations on overlapping star-like topologies. Color code symbolizes movements due to hosts from different nodes.

$N_{m}^{k}$ individuals of species $n$ in the location $k$ for all $n$ and $k$

$$
\begin{aligned}
& \frac{d \mathcal{P}\left(\ldots, N_{n}^{m}, N_{n}^{k}, \ldots\right)}{d t} \\
& \quad=\sum_{n, m, k}\left[\omega_{k m}^{n} N_{n}^{m} \mathcal{P}\left(\ldots, N_{n}^{k}-1, N_{n}^{m}+1, \ldots\right)-\omega_{m k}^{n} N_{n}^{k} \mathcal{P}\left(\ldots, N_{n}^{k}+1, N_{n}^{m}-1, \ldots\right)\right] .
\end{aligned}
$$

For the average number of agents $\left\langle N_{n}^{m}\right\rangle \equiv N_{n}^{m}=\int N_{n}^{m} d N_{n}^{m} \mathcal{P}\left(\ldots, N_{n}^{m}, \ldots\right)$ belonging to the location $n$ holds

$$
\frac{d N_{n}^{k}(t)}{d t}=\sum_{k, m}\left[\omega_{k m}^{n} N_{n}^{m}(t)-\omega_{m k}^{n} N_{n}^{k}(t)\right] .
$$

This equation represents a generalized description of the individual movements with the origindependent transition rates. Here, we neglected the possibility for individuals to change their home location, which can be easily incorporated in our model by introducing the reaction for the change of the corresponding bottom index

$$
N_{n}^{k} \underset{\Omega_{k}^{m m}}{\stackrel{\Omega_{k}^{m n}}{\rightleftarrows}} N_{m}^{k}
$$

where by $\Omega_{k}^{m n}$ denotes the rate of change of the home location from the old location $n$ to the new location $m$, being in the location $k$. If we discard the recurrent movements and consider only the reaction (4.1) we recover the usual random walk movement pattern of indistinguishable individuals.

Thus, we built the general model for the travel with transition rates, dependend on the location of species origin. We apply our formalism to the special case of the recurrent movements on star-like topologies and investigate its properties (Section 4.1.1). In Section 4.1.2 we will introduce interaction between different species on the example of infectious dynamics.

\subsubsection{Recurrent movements on star-like topologies}

We focus on the case of individuals moving only between their home location and neighboring ones, with the assumption that, before individuals move to some non-home location they 
always return home. Individuals commute between their home and other locations, i.e. every individual performs a generalized random telegraph process (Gardiner, 1997). Effectively, individuals move on the overlapping star-like topologies with a home location representing the proximal node and links to neighbouring, distal locations representing the edges (Figure 4.2(c)) In contrast to the diffusive models, in which the probability to return to the origin location may be even less than unity (a transient process) (Feller, 1971), our processes are always recurrent. We will refer to such a situation as the coupling due to recurrent movements or the bidirectional coupling, as opposed to the direct coupling and the random walk (diffusive) mediation of the epidemic spread (Chapter 2).

To describe the above mentioned situation we need to impose restrictions on the travel rates of such kind

$$
\omega_{k m}^{n}=\omega_{n m}^{n} \delta_{k n}+\omega_{k n}^{n} \delta_{m n} .
$$

$\delta_{m n}$ denotes the Kroneker delta: $\delta_{n m}=0$ if $m \neq n$ and $\delta_{n m}=1$ if $m=n$. This implies that one of the bottom indices of travel rates has to coincide with the upper denoting the home location. The traveling is explicitly described by the following reaction scheme

$$
N_{n}^{n} \underset{\omega_{n m}^{n}}{\stackrel{\omega_{m n}^{n}}{\rightleftharpoons}} N_{n}^{m}
$$

with the corresponding mean-field equations

$$
\frac{d}{d t} N_{n}^{m}=\delta_{m n} \sum_{k \in \Delta_{n}}\left(\omega_{n k}^{n} N_{n}^{k}-\omega_{k n}^{n} N_{n}^{n}\right)+\left(1-\delta_{m n}\right)\left(\omega_{m n}^{n} N_{n}^{n}-\omega_{n m}^{n} N_{n}^{m}\right)
$$

with $N_{n}^{m}=N_{n}^{m}(t)$ and where $\Delta_{n}$ lists all neighbours of the $n$-th location.

\subsubsection{Stationary properties}

From the above equations, it follows that a number of individuals in the $n$-th location is not necessarily conserved. If initially, all individuals were in their home locations $N^{n}(t=0)=$ $N_{n}, \forall n$ in the course of time, generally $N^{n}(t) \neq N_{n}$, where $N^{n}$ denotes the total number of individuals being in the $n$-th location (actual size) and $N_{n}$ denotes the total number of individuals having the $n$-th location as a home (nominal size). Equation (4.3) also implies that generally $N_{m}^{n} \neq N_{n}^{m}$, i.e. the number of species $n$ sojourning in the location $m$ may not equal exactly to the number of species $m$ sojourning in location $n$. Let us calculate the stationary actual population sizes. At equilibrium, the detailed balance (Gardiner, 1997) for the reaction (4.2) is fulfilled:

$$
\omega_{m n}^{n} N_{n}^{n \star}=\omega_{m n}^{n} N_{n}^{m \star},
$$

where an asterisk denotes equilibrium quantities. Hence, one can write down the equilibrium number of individuals from population $n$ sojourning in location $m$ in terms of individuals remaining at home

$$
N_{n}^{m \star}=\frac{\omega_{m n}^{n}}{\omega_{n m}^{n}} N_{n}^{n \star}
$$


Thus, the ratio of $\omega_{n m}^{n}$ and $\omega_{m n}^{n}$ is given by the fraction of the number of individuals staying in their home location to the number of individuals having left it to go to another location $m$. Hence, the tensor of transition rates $\omega_{n m}^{n}$ can be defined operationally using the stationary properties of the model. Without a loss of generality, we can assume, that there are no birthdeath dynamics in the population. Therefore $N_{n}$ - the total population of the species $n-$ remains constant for all $n$. From the conservation condition $\sum_{m} N_{n}^{m}=N_{n}$, it follows that

$$
N_{n}=N_{n}^{n \star}\left(1+\sum_{m \neq n} \frac{\omega_{m n}^{n}}{\omega_{n m}^{n}}\right) \quad \text { and thereby } N_{n}^{n \star}=N_{n}\left(1+\sum_{m \neq n} \frac{\omega_{m n}^{n}}{\omega_{n m}^{n}}\right)^{-1} .
$$

Hence, the expression of the equilibrium actual population size of the location $n$ reads

$$
N^{n \star}=\sum_{m \neq n} \frac{\left[\delta_{n m}+\left(1-\delta_{m n}\right) \frac{\omega_{m m}^{m}}{\omega_{m n}^{m}}\right] N_{m}}{1+\sum_{k \neq m} \frac{\omega_{k m}^{m}}{\omega_{m k}^{m}}} .
$$

We can also define the occupation probability for an individual, belonging to the $n$-th location to be in some location $m$

$$
p_{n}^{m}=\frac{N_{n}^{m \star}}{N_{n}}=\frac{\left[\delta_{n m}+\left(1-\delta_{m n}\right) \frac{\omega_{m n}^{n}}{\omega_{n m}^{n}}\right]}{1+\sum_{k} \frac{\omega_{k n}^{n}}{\omega_{n k}^{n}}}
$$

We observe that it depends, in a complicated way, on initial population sizes of neighboring locations, and on the backward and the forward transition rates. In this way, topology of the metapopulation comes into play. For example, assuming equal transition rates $\omega_{m n}^{n}=\omega_{n m}^{n}$, we obtain

$$
N^{n \star}=\sum_{m \in\left\{n, \Delta_{n}\right\}} \frac{N_{m}}{1+k_{m}},
$$

where $k_{n}$ is a number of neighboring locations for the location $n$, i.e. its node degree, in the metapopulation network (See also discussions in Section 4.6 on network topologies).

Our assumption of the constant travel rates leads to the uniform distribution of species over the their home locations and neighbor nodes, e.g. for the $n$-th species: $N_{n}^{m \star}=N_{n}^{n \star}$. Individuals also spend an equal amount of time in their home and neighbour locations. It is reasonable to assume $N_{n}^{m \star} / N_{n}^{n \star} \ll 1$ in many situations, which implies that individuals belonging to the $n$-th location remain mostly there. Instead of the assumption of constant transition rates we consider the fixed ratio of the forward $\omega_{m n}^{n}=\omega_{1}$ to the backward $\omega_{n m}^{n}=\omega_{2}$ rates

$$
\varepsilon=\frac{\omega_{1}}{\omega_{2}} .
$$

From (4.4), it follows that equation (4.7) gives us the ratio of the number $N_{n}^{m \star}$ of individuals of the species $n$ sojourning in the $m$-th location to the number $N_{n}^{n \star}$ of the individuals staying at home $\left(\varepsilon=N_{n}^{m \star} / N_{n}^{n \star}\right)$. In the following, we will call $\varepsilon$ the commuter ratio. We will discuss other possibilities for the choice of travel rates in Section 4.6.1, where we assume proportionality of the forward transfer rates to the size or capacity of the target location. Note, 
that depending on travel rates, different approximations, e.g. such as the direct coupling one (see section 4.2), can be made. However, before we start a comparison of presented formalism to other models, we need to incorporate species interaction.

\subsubsection{Interacting species}

After having introduced the recurrent travel mechanism for spatial dynamics, we can combine it with different local kinetics. In what follows, without a loss of generality, we will use the SIS epidemiological model (see Chapter 2) assuming locally a well mixed population. Note, that nothing hinders us from considering other, more comprehensive models (Anderson and May, 1991). In the SIS model we need to consider two classes of individuals: infectives and susceptibles. We denote by $I_{n}^{m}$ and by $S_{n}^{m}$ the number of infectives and susceptibles respectively, which belong to the location $n$ (bottom index) and are currently sojourning in the location $m$ (upper index). Thus, infectives from every location may meet susceptibles from generally every location. Now we introduce the aforementioned recurrent travel mechanism. Individuals may leave their home location $n$ and travel to some other location $m$ with the rate $\omega_{m n}^{n}$, and also return with the rate $\omega_{n m}^{n}$. This leads to the following set of reactions $\forall n, m, k$ :

$$
\begin{aligned}
& I_{m}^{n}+S_{k}^{n} \stackrel{\alpha}{\rightarrow} I_{m}^{n}+I_{k}^{n} \\
& I_{n}^{m} \stackrel{\beta}{\rightarrow} S_{n}^{m} \\
& I_{n}^{n} \underset{\omega_{n m}^{n}}{\stackrel{\omega_{m n}^{n}}{\rightleftharpoons}} I_{n}^{m} \\
& S_{n}^{n} \underset{\omega_{n m}^{m}}{\stackrel{\omega_{m n}^{n}}{\rightleftharpoons}} S_{n}^{m} .
\end{aligned}
$$

The model is illustrated in Figure 4.1. Different colors correspond to individuals belonging to different locations. The mean-field equations corresponding to the system (4.8) read

$$
\begin{aligned}
\frac{d}{d t} I_{n}^{n} & =\frac{\alpha}{N^{n}} S_{n}^{n} \sum_{m} I_{m}^{n}-\beta I_{n}^{n}+\sum_{m}\left(\omega_{n m}^{n} I_{n}^{m}-\omega_{m n}^{n} I_{n}^{n}\right) \\
\frac{d}{d t} S_{n}^{n} & =-\frac{\alpha}{N^{n}} S_{n}^{n} \sum_{m} I_{m}^{n}+\beta I_{n}^{n}+\sum_{m}\left(\omega_{n m}^{n} S_{n}^{m}-\omega_{m n}^{n} S_{n}^{n}\right) \\
\frac{d}{d t} I_{n}^{m} & =\frac{\alpha}{N^{m}} S_{n}^{m} \sum_{k} I_{k}^{m}-\beta I_{n}^{m}+\omega_{m n}^{n} I_{n}^{n}-\omega_{n m}^{n} I_{n}^{m} \quad \forall n \neq m \\
\frac{d}{d t} S_{n}^{m} & =-\frac{\alpha}{N^{m}} S_{n}^{m} \sum_{k} I_{k}^{m}+\beta I_{n}^{m}+\omega_{m n}^{n} S_{n}^{n}-\omega_{n m}^{n} S_{n}^{m} \quad \forall n \neq m,
\end{aligned}
$$

where index $m$ may take every value $m=1, \ldots, M$ and $N^{n}$ is the actual size of the location $n$. Note that similar equations were proposed in parallel to us in (Sattenspiel and Dietz, 1995). In the worst case of all-to-all coupling, the number of Equations (4.9) is $2 \times M^{2}$. One can reduce this number to $2 \times M^{2}-M$ taking into account conservation of the populations belonging to 
the location $n: \sum_{m}\left(I_{n}^{m}+S_{n}^{m}\right)=$ const. However, there are still too many equations to solve as compared with reaction-diffusion or directly coupled SIS models, where just $2 \times M$ dynamical quantaties need to be considered. Thus, to incorporate the fact that individuals always return back, we need to expand dynamical dimension of our system dramatically. However, locations need not be all-to-all coupled and the number of equations can sometimes be reduced significantly. In the simple case of one-dimensional topology, we can derive a continuum limit of the corresponding equations and obtain some important analytical results concerning e.g. the speed of the epidemic wave (Section 4.4).

One can also reformulate Equations (4.9) from the point of view of each location, i.e. consider the total numbers of infectives and susceptibles in one particular location: $I^{n}=\sum_{k} I_{k}^{n}$ and $S^{n}=\sum_{k} S_{k}^{n}$. We obtain e.g. for infectives

$$
\frac{d}{d t} I^{n}=\frac{\alpha}{N^{n}} S^{n} I^{n}-\beta I^{n}+\sum_{k \neq n}\left(\omega_{n k}^{k} I_{k}^{k}+\omega_{n k}^{n} I_{n}^{k}-\omega_{k n}^{k} I_{k}^{n}-\omega_{k n}^{n} I_{n}^{n}\right) .
$$

The dynamics of the total number of infectives in the location $n$ can be separated into the fully mixed reaction part and transport part ${ }^{1}$. However, the last part cannot be reduced to neither the reaction-diffusion nor to the directly coupled model without further assumptions (Section 4.2).

The aforementioned recurrent transport mechanism can also play a role in other, not only epidemiological, spreading phenomena (see Section4.7). The epidemic dynamics of species interaction was considered just as an important example of processes which involve the recurrent movements.

\subsection{Direct versus bidirectional coupling}

Epidemiologists have long understood that the effective diffusion-mediated coupling is not a good ansatz to model modern human epidemics. If humans travel, i.e. move on large scale among different cities, they do not perform a random walk but rather go back and forth between some permanent location and some temporary ones. This leads to their effective presence on several locations at the same time. Therefore, it is rather straightforward to phenomenologically assume that the infectious incidence ${ }^{2}$ in one location is proportional to the prevalence ${ }^{3}$ in other locations. Adopting the local SIS dynamics, one can write down the following equations dating back to (Rushton and Mautner, 1955)

$$
\frac{d}{d t} I_{n}=\frac{\alpha}{N_{n}} S_{n} \sum_{m} \epsilon_{n m} I_{m}-\beta I_{n}
$$

${ }^{1}$ The same holds for the general model with origin-dependent transition rates and the dynamics of the actual number of infectives in location $n$ given by the equation

$$
\frac{d I^{n}}{d t}=\frac{\alpha}{N^{n}} S^{n} I^{n}-\beta I^{n}+\sum_{k, m}\left(\omega_{n k}^{m} I_{m}^{k}-\omega_{k n}^{m} I_{m}^{n}\right) .
$$

${ }^{2}$ the rate of change of the number of infectives

${ }^{3}$ number of infectives in one location 
where $I_{n}, S_{n}$ are infectives and susceptibles from the location $n$ and $\epsilon_{n m}$ are coupling strengths. We call this approach the direct coupling model (see also Chapter 2, Section 2.4).

Let us show that equation (4.10) follows from our approach described by the system (4.9), under the assumption of large travel rates as compared to the intrinsic epidemic time scale, i.e.

$$
\omega_{m n}^{n} \sim \omega_{n m}^{n} \gg \frac{\alpha}{\beta}
$$

The last inequality implies that the time scales of the reaction and the transport are clearly separated. In this case, the equilibration with respect to transport occurs almost immediately and one can neglect the last terms in (4.9) responsible for the transport in equations (4.9). Note, that in parallel to us some of our findings concerning the scale separation were anticipated in (Keeling and Rohani, 2007). We consider only infectives. One can proceed analogously for susceptibles . Using the assumption (4.11), we obtain

$$
\omega_{n m}^{n} I_{n}^{m}=\omega_{m n}^{n} I_{n}^{n}
$$

hence, follows

$$
I_{n}^{m}=\frac{\left[\delta_{n m}+\left(1-\delta_{n m}\right) \frac{\omega_{m n}^{n}}{\omega_{n m}^{n}}\right] I_{n}}{1+\sum_{k} \frac{\omega_{k m}^{m}}{\omega_{m k}^{m}}}=p_{n}^{m} I_{n},
$$

where $p_{n}^{m}$ is an occupation probability to find an individual of species $n$ in the location $m$ already defined by Equation (4.5)

$$
p_{n}^{m}=\frac{\left[\delta_{n m}+\left(1-\delta_{m n}\right) \frac{\omega_{m n}^{n}}{\omega_{n m}^{n}}\right]}{1+\sum_{k} \frac{\omega_{k n}^{n}}{\omega_{n k}^{n}}} .
$$

In Equation (4.9), we sum over index $m$ denoting the current location and obtain

$$
\frac{d}{d t} \sum_{m} I_{n}^{m}=\sum_{m} \frac{\alpha}{N^{m}} S_{n}^{m} \sum_{k} I_{k}^{m}-\beta \sum_{m} I_{n}^{m} .
$$

Utilizing Equation (4.12), we can write

$$
\frac{d}{d t} I_{n}=\alpha S_{n} \sum_{k}\left(\sum_{m} \frac{p_{n}^{m} p_{k}^{m}}{N^{m}}\right) I_{k}-\beta I_{n} .
$$

Comparing the last equation with Equation (4.10) we see that

$$
\epsilon_{n m}=\frac{p_{n}^{m} p_{k}^{m}}{N^{m}}
$$

Note that the coupling strength is not just directly proportional to corresponding travel rates as it was assumed in (Camitz and Liljeros, 2006), but rather depends on them in a complicated manner (cf. Equations (4.14) and (4.13)). In addition, for the genuine direct coupling model 

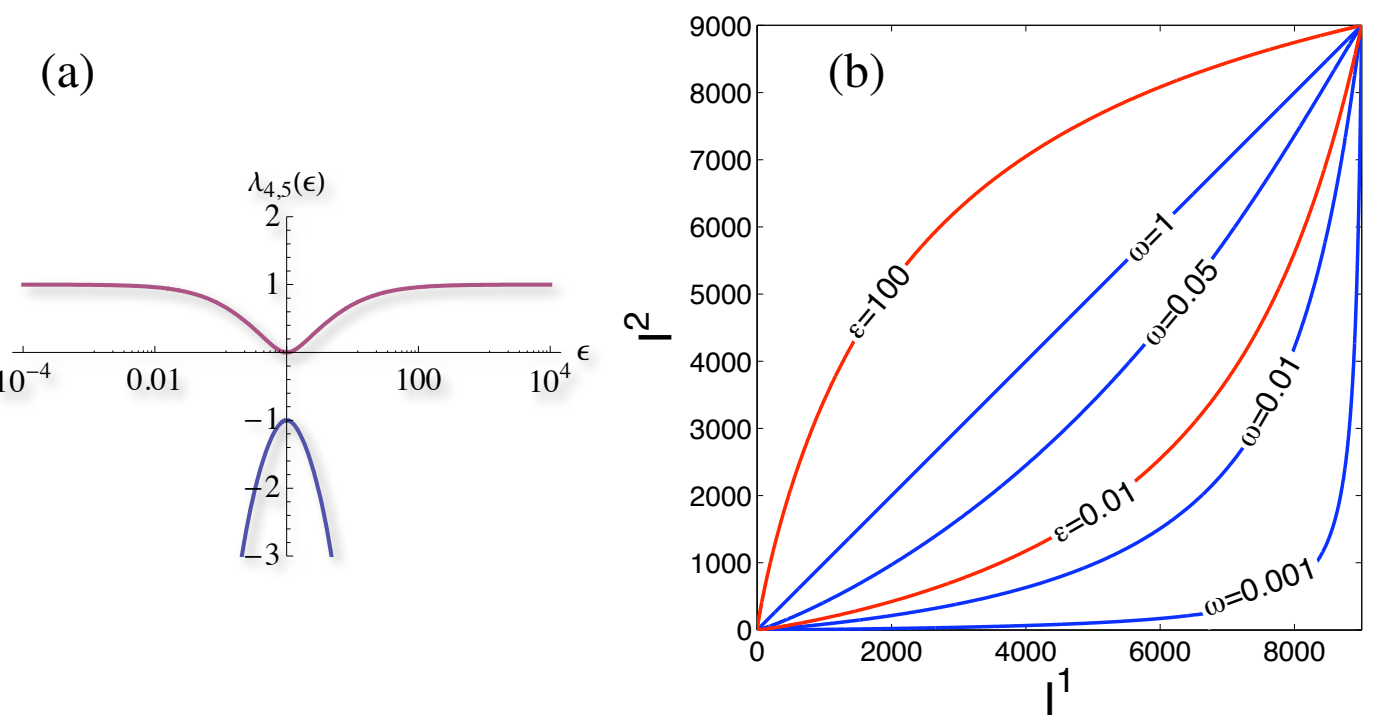

Figure 4.3: Panel (a): Dependence of $\lambda_{4}(\varepsilon)$ and $\lambda_{5}(\varepsilon)$ eigenvalues (blue and red colour respectively) of the linearized near the unstable fix-point system (4.16) on the ratio of the travel rates $\varepsilon=\omega_{1} / \omega_{2}$. One observes the minimum of the non-negative eigenvalue $\lambda_{4}$ at $\varepsilon=1$ leading to the most pronounced coupling of two locations. Panel (b): Projection of the phase space of the system (4.16) on the $\left(I^{1}, I^{2}\right)$ plane. Blue lines correspond to the case $\omega_{1}=\omega_{2}=\omega$ and thus $\varepsilon=1$ which is equivalent to the system (4.15). One observes greater coupling with increasing travel rate $\omega$. Red lines depict trajectories for $\omega=1$ and $\omega_{1,2}$ calculated from (4.17) for two values of $\varepsilon=0.01$ and $\varepsilon=100$. One observes the greatest coupling for $\varepsilon=1$. Parameters of the SIS model: $\alpha=1$ and $\beta=0.1$.

to be valid, together with the low travel rates one should implicitly assume that only a small fraction of individuals is travelling, so that the actual population of the location $n$ could be approximated by the nominal one $N^{n} \approx N_{n}$. Compare also discussion in Section 4.1.1. A small fraction of travelling individuals implies $\omega_{n m}^{n} \ll \omega_{m n}^{n}$.

Thus, the widely-used approach of direct coupling is a particular case of our general model valid in the case of high travel rates. The coupling strength is then given by Equation (4.14), where probabilities of being at some particular locations are given by Equation (4.5). Therefore, we established the relation between the directly coupling approach and the underlying travel pattern from first principles as well as assessed a validity range of the direct coupling model.

\subsection{Exemplary case of two populations}

Let us consider recurrent host movements in the simplest possible case of just two locations. Individuals belonging to one of them can move to another, but always return to their home location, where they spend a fraction of time which is usually larger than in the non-home location. That stands in contrast to random walk movements, in which individuals do not prefer one particular location and spend equal amounts of time in both locations.

The equations governing the dynamics of SIS epidemics in the case when we distinguish 
individuals according to where they currently are (the random walk based model) read

$$
\begin{aligned}
\frac{d}{d t} I_{1} & =\frac{\alpha}{N_{1}} S_{1} I_{1}-\beta I_{1}+\omega\left(I_{2}-I_{1}\right) \\
\frac{d}{d t} S_{1} & =-\frac{\alpha}{N_{2}} S_{1} I_{1}+\beta_{1} I_{1}+\omega\left(S_{2}-S_{1}\right)
\end{aligned}
$$

and analogous equations hold for the second location. $\omega$ denotes the travel rate. Note that because of conservation of the total number of individuals in both locations we can get rid of one variable. In the following, however, we consider the full system of 4 equations.

If we distinguish between individuals not just according to their current location, but whether they originate from population 1 or 2 (model based on recurrent movements) equations read

$$
\begin{aligned}
\frac{d}{d t} I_{1}^{1} & =\frac{\alpha}{N^{1}} S_{1}^{1}\left(I_{1}^{1}+I_{2}^{1}\right)-\beta I_{1}^{1}+\omega_{2} I_{1}^{2}-\omega_{1} I_{1}^{1} \\
\frac{d}{d t} I_{1}^{2} & =\frac{\alpha}{N^{2}} S_{1}^{2}\left(I_{1}^{2}+I_{2}^{2}\right)-\beta I_{1}^{2}+\omega_{1} I_{1}^{1}-\omega_{2} I_{1}^{2} \\
\frac{d}{d t} S_{1}^{1} & =-\frac{\alpha}{N^{1}} S_{1}^{1}\left(I_{1}^{1}+I_{2}^{1}\right)+\beta I_{1}^{1}+\omega_{2} S_{1}^{2}-\omega_{1} S_{1}^{1} \\
\frac{d}{d t} S_{1}^{2} & =-\frac{\alpha}{N^{2}} S_{1}^{2}\left(I_{1}^{2}+I_{2}^{2}\right)+\beta I_{1}^{2}+\omega_{1} S_{1}^{1}-\omega_{2} S_{1}^{2}
\end{aligned}
$$

and analogous equations can be written for the second population. We have 8 equations in total. We assume here that populations are equilibrated in respect to travel dynamics and thus $N^{1}$ and $N^{2}$ can be assessed. We consider the case of equal population sizes $N_{1}=N_{2}=N$. One can show that systems (4.15) and (4.16) are equivalent in the case $\omega=\omega_{1}=\omega_{2}$. This is intuitively clear: if agents are equally distributed between two (or even three) locations, it does not matter if we distinguish between them according to their origin or not. That is not true anymore if we consider the non-equal distribution of agents, which can only occur if forward and backward rates are non-equal: $\omega_{1} \neq \omega_{2}$. The commuter ratio $\varepsilon=\omega_{1} / \omega_{2}$ introduced in Section 4.1.1 yields the ratio of the time spent by agents from one location in another one $\tau_{2}$ to the time spent in their home location $\tau_{1}: \varepsilon=\tau_{2} / \tau_{1}$. If $\varepsilon=1$, we recover the case of equal distribution.

The consequences of $\varepsilon \neq 1$ can be understood by means of a linear stability analysis. Namely, if one expands the system (4.16) according to the Taylor formula near the unstable fix point, the system (4.16) takes the form $d_{t} X=A \delta X$, where $X=\left\{I_{1}^{1}, I_{1}^{2}, S_{1}^{1}, S_{1}^{2}, I_{2}^{2}, I_{2}^{1}, S_{2}^{2}, S_{2}^{1}\right\}$. If we assume an exponential as the solution of the linear system $\delta X \sim e^{\lambda t}$, the eigenvalues of the matrix $A$ determine the dynamics near the fixed point $X=\left\{0,0, \frac{N}{1+\varepsilon}, \frac{\varepsilon N}{1+\varepsilon}, 0,0, \frac{N}{1+\varepsilon}, \frac{\varepsilon N}{1+\varepsilon}\right\}$. The eigenvalues read: $\lambda_{1}=0$ (threefold degenerated), $\lambda_{2}=\alpha, \lambda_{3}=-\omega_{1}-\omega_{2}$ (threefold degenerated) with $\lambda_{4}$ and $\lambda_{5}$ skipped because of the complicated expressions for them in a general case.

Note that in the case of the equal rates we recover the eigenvalues of the system (4.15) $\Lambda=\{0, \alpha,-2 \omega, \alpha-2 \omega\}$. To compare behavior of the system (4.16) with (4.15) for $\varepsilon \neq 1$, we calibrate both systems so that the flow of agents between both location remains the same. That implies

$$
\omega_{1}=\frac{1+\varepsilon}{2} \omega \text { and } \omega_{2}=\frac{1+\varepsilon}{2 \varepsilon} \omega .
$$


In the case $\alpha=1$ and $\omega=1$, the dependence $\lambda_{4,5}(\varepsilon)^{4}$ is depicted in Figure 4.3(a). Generally we have a many-dimensional saddle point, but how fast the instability wins is determined by the positive eigenvalues. $\lambda_{4}(\varepsilon)$ is always positive, except for $\varepsilon=1$, where it takes the minimal value - zero. Maximal values are achieved for very small and very large values of $\varepsilon . \lambda_{5}(\varepsilon)$ is always negative. For $\varepsilon<1$, it is large by absolute value and achieves maximum for $\varepsilon \rightarrow 1$ from below. For $\varepsilon>1$, it rapidly grows by the absolute value with $\varepsilon$. $\lambda_{5}(1)=\alpha-2 \omega$ and equals one of the eigenvalues of the system (4.15). This implies, that at $\varepsilon \approx 1$, the trajectory in the phase space projection on $\left(I^{1}, I^{2}\right)$-plane at the least deviate from the diagonal leading to the most pronounced synchronisation or coupling of both locations (Figure 4.3(b)). $\varepsilon=1$ corresponds to the case when individuals spend their time equally on both locations, cf. (Keeling and Rohani, 2005). With changing $\varepsilon$, we could obtain retardation of the epidemic in both locations: for $\varepsilon<1$ in the second (there are too few travellers to transfer the disease into the second location) and for $\varepsilon>1$ in the first (there are too many travellers and the disease is first transferred into the second location before it develops in the first one). Thus, if the backward and forward travel rates are equal, the linear stability analysis reveals no difference between dynamics mediated by random walk and recurrent movements. The case of different backward and forward rates is illustrated in Figure 4.3(b) as well.

\subsection{Epidemics on continuum support}

Under particular conditions a set of partial differential equations can be derived from a set of ordinary differential equations (ODEs) for the spreading process on a support with a regular topology like lattice or chain (Figure 4.4). Obtained equations are called continuum approximation of the original ODEs. They can usually be easier analysed than the original ODEs due to the uncoupling of the dynamical quantities corresponding to different locations. In many reaction-diffusion systems the continuum limit have revealed a lot of important properties of the epidemic spread, e.g. velocity of the epidemic front or front shape. As already mention in Chapter2, the FKKP reaction-diffusion model (Kolmogorov et al., 1937; Fisher, 1937) was initially introduced in order to describe the advance of the diffusing gene in space; however, it describes the spread of the SI epidemic as well:

$$
\partial_{t} u=\mathcal{D} \Delta u+\alpha(1-u) u
$$

Continuum equations for the epidemic spread in the case of recurrent and confined host movements were a challenge until recently. In what follows, we will propose an approach allowing us to easily formulate corresponding continuum limit equations which are still analytically treatable.

\subsubsection{Epidemics due to recurrent transport}

Let us consider an homogeneous lattice with locations as its nodes depicted in Figure 4.4. We study the SI model with recurrent movements (without recovering: $\beta=0$, see also Chapter 2)

${ }^{4} \lambda_{4,5}(\varepsilon)=-\frac{1+\varepsilon+\varepsilon^{2}+\varepsilon^{3} \pm \sqrt{(1+\varepsilon)\left(1+8 \varepsilon-14 \varepsilon^{2}+8 \varepsilon^{3}+\varepsilon^{4}\right)}}{4 \varepsilon(1+\varepsilon)}$ 


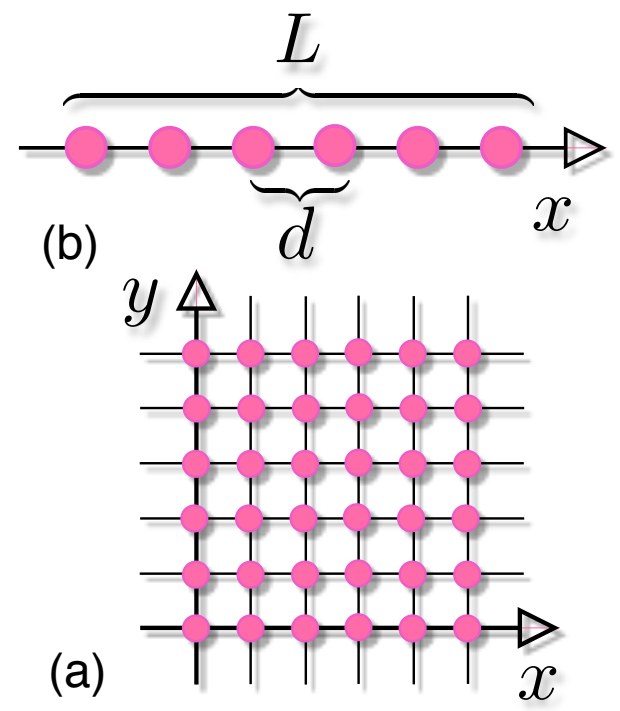

Figure 4.4: A scheme of lattice (a) or chain (b) model. Every circle represents a location with its own population. Individuals are allowed to travel among different locations either by the random walk pattern or recurrently (cf. Figure 4.2).

on the lattice with the next-neighbour coupling. For simplicity, we assume that only infectives can travel. We call this model the SI0 model. Although this seems artificial, it is the only way to reduce the dimensionality of the dynamical system. As we will see later, this assumption does not significantly change our results. We cannot allow susceptibles in place of infectives to travel. This is because, if they become infectives not in their home location we should assume either that they stay at their current location or that the infectives travel as well. Without loss of generality, we consider the epidemic on a one-dimensional lattice. Let us denote the number of invectives staying at home in location $n$, by $I_{n}^{n}$. The number of infectives being elsewhere at the moment is denoted by $I_{n}^{-}$and $I_{n}^{+}$commuting respectively to $(n-1)$ th and $(n+1)$ th location. Hence, analogously to Equations (4.9), we can write down the following reactions

$$
\begin{array}{rll}
I_{n}^{n}+S_{n} & \stackrel{\alpha}{\longrightarrow} 2 I_{n}^{n} \\
I_{n \mp 1}^{ \pm}+S_{n} & \stackrel{\alpha}{\longrightarrow} I_{n \mp 1}^{ \pm}+I_{n}^{n} \\
I_{n}^{n} & \underset{\omega_{2}}{\stackrel{\omega_{1}}{\rightleftarrows}} I_{n}^{ \pm},
\end{array}
$$

where $S_{n}$ denotes the number of susceptibles in the $n$-th location. The system of reactions (4.19) leads to the following mean field equations

$$
\begin{aligned}
\frac{d}{d t} I_{n}^{n} & =\frac{\alpha}{N}\left(N-I_{n}^{n}-I_{n}^{+}+I_{n}^{-}\right)\left(I_{n}^{n}+I_{n-1}^{+}+I_{n+1}^{-}\right)+\omega_{2}\left(I_{n}^{+}+I_{n}^{-}\right)-2 \omega_{1} I_{n}^{n} \\
\frac{d}{d t} I_{n}^{ \pm} & =\omega_{1} I_{n}^{n}-\omega_{2} I_{n}^{ \pm}
\end{aligned}
$$

where we have assumed that the size of one location remains constant during epidemic and equals $N$, which is true for a homogeneous lattice. If the dynamics in the neighbor locations is similar enough, i.e. $\frac{I_{n-1}-I_{n}}{I_{n}} \ll 1$, we can make use of the Taylor expansion $I^{ \pm}(x) \approx$ $I^{ \pm}(x) \pm d \nabla I^{ \pm}+\frac{d^{2}}{2} \Delta I^{ \pm}$, where we can introduce a continuous space variable $x$. Hence, 
follows

$$
\begin{aligned}
\partial_{t} I(x, t)= & \frac{\alpha}{N}\left\{\left(N-I-I^{+}-I^{-}\right)\left[I+I^{+}+I^{-}+C \nabla\left(I^{-}-I^{+}\right)+D \Delta\left(I^{+}+I^{-}\right)\right]\right\} \\
& +\omega_{2}\left(I^{+}+I^{-}\right)-2 \omega_{1} I \\
\partial_{t} I^{ \pm}(x, t)= & \omega_{1} I-\omega_{2} I^{ \pm},
\end{aligned}
$$

where $C=d, D=d^{2} / 2$ and $d$ is the distance between locations. If we consider new variables $u=I / N$ and $v=\left(I^{+}+I^{-}\right) / N$, we obtain

$$
\begin{aligned}
\partial_{t} u & =\alpha(1-u-v)(u+v+C \nabla w+D \Delta v)+\omega_{2} v-2 \omega_{1} u \\
\partial_{t} v & =2 \omega_{1} u-\omega_{2} v \\
\partial_{t} w & =-\omega_{2} w .
\end{aligned}
$$

Equations (4.21) are structurally different from the FKPP one (4.18). Reaction and diffusion are not uncoupled anymore. From the third equation, for $w$, we obtain $w(x, t)=w(x, 0) e^{-\omega_{2} t}$. This implies that $w$ depends only on the initial conditions and disappears quickly especially for large $\omega_{2}$. Thus, we can discard the gradient term in (4.21), which, as it can be shown, does not influence our results for the front velocity and we can consider instead of the system (4.21) only two equations:

$$
\begin{aligned}
\partial_{t} u & =\alpha(1-u-v)(u+v+D \Delta v)+\omega_{2} v-2 \omega_{1} u \\
\partial_{t} v & =2 \omega_{1} u-\omega_{2} v
\end{aligned}
$$

The steady states are $\left(u^{\star}, v^{\star}\right)=(0,0)$ and $\left(u^{\star}, v^{\star}\right)=\left(\omega_{2} /\left(2 \omega_{1}+\omega_{2}\right), 2 \omega_{1} /\left(2 \omega_{1}+\omega_{2}\right)\right)$. Thus, in the second endemic steady state, the density of infectives in one city is $u+v=1$, i.e. remains the same as in an isolated population. In fact, the density of infectives equals $u(x, t)$ plus infectives belonging to neighbour locations being now on site $x$. However, in the homogeneous situation, it equals $u(x, t)+v(x, t)$. Thus the recurrent transport does not affect the fix point in homogeneous case of regular lattices.

In the case of high and comparable forward and backward travel rates from equations (4.22) it follows that $v=2 \omega_{1} u / \omega_{2}$ and thus, we have only one equation describing the epidemic

$$
\partial_{t} u=\alpha(1-u) D \Delta u+\alpha(1-u) u,
$$

where we substituted $u \kappa \rightarrow u, \alpha \kappa \rightarrow \alpha$ and $2 D \omega_{1} /\left(\omega_{2} \kappa\right) \rightarrow D$ with $\kappa=1+2 \omega_{1} / \omega_{2}$. From the detailed balance condition, it is clear the new $u(x, t)$ gives us the total concentration of infectives belonging to the particular location $x$. For a homogeneous chain, it gives, at the same time, the actual concentration of infectives. Thus, we can recover the equation (4.39), which is derived as the continuum limit of the directly coupled model valid in the case of high travel rates (see Section 4.2).

We can further investigate Equation (4.22) to find whether this system of equations allows a travelling wave solution and determine the front velocity by the linear stability analysis of the zero steady state. 


\subsubsection{Front velocity}

According to the general scheme of analysis of reaction-diffusion equations (Murray, 1993), we look for traveling wave solution of the system (4.22): $u(x, t)=U(x-c t)$ and $v(x, t)=$ $V(x-c t)$. We obtain

$$
\begin{aligned}
{\left[\alpha(1-U-V) D \frac{2 \omega_{1}}{c}-c\right] U^{\prime}=} & \alpha(1-U-V)\left[U+V+\frac{D \omega_{2}}{c^{2}}\left(\omega_{2} V-\omega_{1} U\right)\right] \\
& +\omega_{2} V-2 \omega_{1} U \\
V^{\prime}= & \frac{1}{c}\left(\omega_{2} V-2 \omega_{1} U\right) .
\end{aligned}
$$

Note that because the left hand side of the system is singular, it is of a differential-algebraic type (Stoer and Bulirsch, 2002; Hairer et al., 2000, 2002) and is not trivial to solve, even numerically. However since we are interested in the stability analysis of the disease-free steady state, this does not matter. To get rid of the prefactor before $U^{\prime}$, which would lead to singularity, we perform the change of variable $z=x-c t \rightarrow \zeta$, such that $\frac{d}{d \zeta}=\left[\alpha D \frac{2 \omega_{1}}{c}(1-U-V)-c\right] \frac{d}{d z}$ and hence

$$
\begin{aligned}
U^{\prime} & =\alpha(1-U-V)\left[U+V+\frac{D \omega_{2}}{c^{2}}\left(\omega_{2} V-2 \omega_{1} U\right)\right]+\omega_{2} V-2 \omega_{1} U \\
V^{\prime} & =\left(\omega_{2} V-2 \omega_{1} U\right)\left[\alpha D \frac{2 \omega_{1}}{c}(1-U-V)-c\right] .
\end{aligned}
$$

The Jacobian of the linearized equations at zero steady state $\left(u^{\star}, v^{\star}\right)=(0,0)$ reads

$$
A=\left(\begin{array}{cc}
\alpha\left(1-D \frac{2 \omega_{1} \omega_{2}}{c^{2}}\right)-2 \omega_{1} & \alpha\left(1+D \frac{\omega_{2}^{2}}{c^{2}}\right)+\omega_{2} \\
-2 \omega_{1}\left(\alpha D \frac{2 \omega_{1}}{c^{2}}-1\right) & \omega_{2}\left(\alpha D \frac{2 \omega_{1}}{c^{2}}-1\right)
\end{array}\right)
$$

Solutions of the corresponding secular equations are

$$
\lambda_{ \pm}=\frac{1}{2}\left[\left(\alpha-\omega_{1}-2 \omega_{1}\right)^{2} \pm \sqrt{\left(\alpha-\omega_{2}-2 \omega_{1}\right)^{2}-4 \alpha\left(\omega_{2}+2 \omega_{1}\right)\left(\alpha D \frac{2 \omega_{1}}{c^{2}}-1\right)}\right] .
$$

For the steady state to be stable we should impose non-negativeness of the expression under the radical. This requirement leads to the condition on the front speed:

$$
c \geq \frac{2 \alpha \omega_{1} \sqrt{2 D\left(2+\frac{\omega_{2}}{\omega_{1}}\right)}}{\alpha+\omega_{2}+2 \omega_{1}} .
$$

As in the usual FKPP case, the selection of the front velocity takes place (Murray, 1993; Kessler et al., 1998) and thus the right part of the inequality (4.23) gives us the only possible 
velocity in the finite size systems. Note that we can measure velocity as a number of affected cities pro time.

If we consider equal forward and backward rates $\left(\omega_{1}=\omega_{2}\right)$ from (4.23) we have for the velocity

$$
c=\frac{2 \alpha \omega \sqrt{6 D}}{\alpha+3 \omega} .
$$

This is the case in which individuals spend half of their time in both "permanent" and "temporary" locations. They effectively belong to both their home and neighbor locations. In the case of high travel rates $\omega \gg 1$, the velocity attains the asymptotic value linearly proportional to the infection rate $\alpha$ :

$$
\lim _{\omega \rightarrow \infty} c=\frac{2 \sqrt{6 D}}{3} \alpha .
$$

The epidemic front in the case of random walk model described by the FKKP equation (cf. Fundamentals) possess the velocity (Murray, 1993)

$$
c=2 \sqrt{D \omega \alpha} .
$$

We observe that it grows unboundedly with the travel rate. Thus, in the ordinary case with the random walk movement pattern, we have an ever-growing velocity with a growing travel rate. Contrary to this, in the case of bidirectional movements, the velocity reaches its asymptotic value, i.e. the velocity is bounded from above. This is one of the main consequences of the recurrent transport mechanism for the epidemic spread. We will discuss this in detail in Section 4.5. In the case of high reaction rates (or low travel rates), we obtain from Equation (4.24)

$$
\lim _{\alpha \rightarrow \infty} c=2 \omega \sqrt{6 D}
$$

i.e. linear dependence on the travel rate. In Section 4.4.5, we will see that this limit is an artifact of the continuous description of the species concentration.

Backward and forward travel rates may not be the appropriate quantaties one needs to consider. The more intuitively assessable quantity is the ratio of the population traveling to a particular location relative to the population staying at home

$$
\varepsilon_{n}^{m}=\frac{N_{n}^{m}}{N_{n}^{n}},
$$

which we call the commuter ratio (cf. Section 4.1.1 and 4.3). In homogeneous case: $\varepsilon=$ $\omega_{1} / \omega_{2}$. We can assume the detailed balance in the stationary case, i.e. that the flow of travellers belonging to location $n$ and leaving $n$ is equal to the flow of travellers belonging to location $n$ and arriving at $n$ :

$$
\omega_{1} N_{n}^{n \star}=\omega_{2} N_{n}^{m \star} .
$$

Then, for the total flow between two neighbour locations holds

$$
F_{n m}=\omega_{1} N_{n}^{n}+\omega_{2} N_{m}^{n}
$$

with $m=n \pm 1$. In equilibrium, the detailed balance for the total flows is also fulfilled

$$
F_{n m}^{\star}=F_{m n}^{\star}=\omega N,
$$



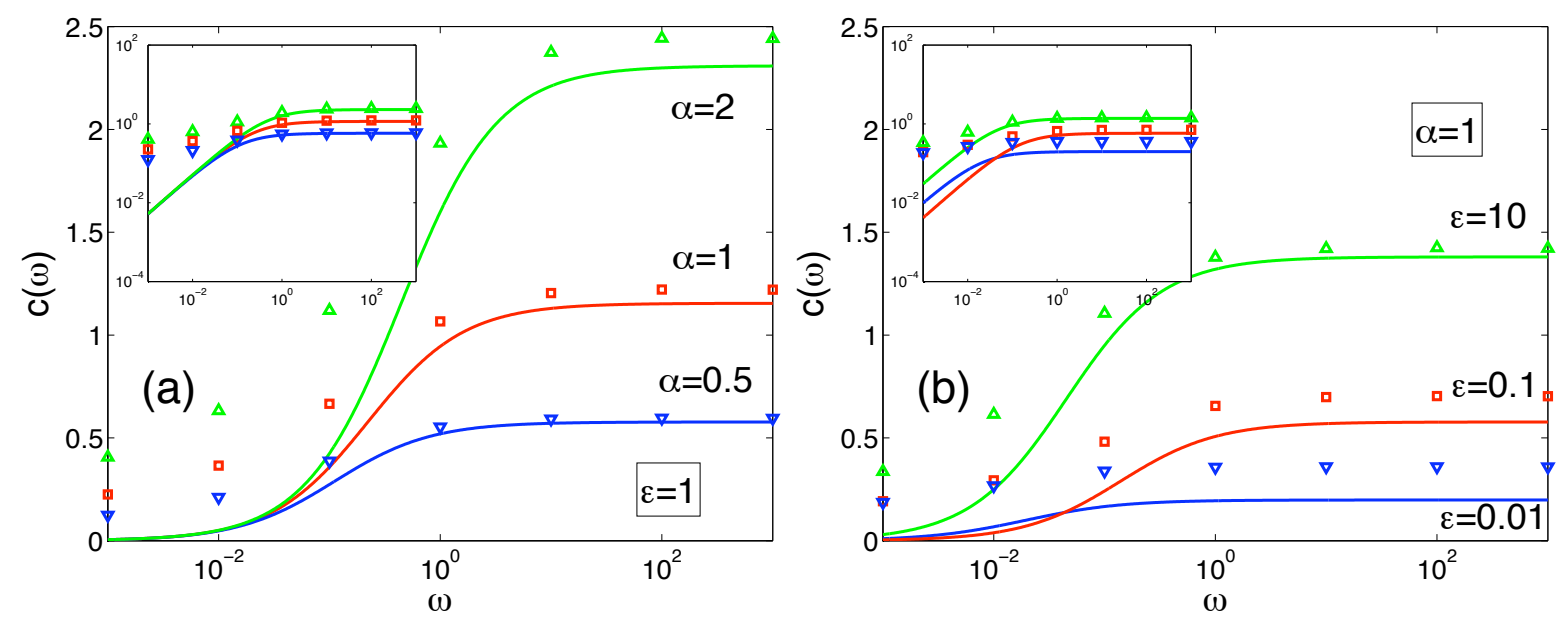

Figure 4.5: Dependence $c(\omega)$ of the front velocity on the travel rate. Curves represent analytical results given by Equation (4.30) (with $D=1 / 2$ ). Symbols repsresent results of the numerical solution of the system of ODEs (4.20). Panel (a): fixed commuter ratio $\varepsilon$. Panel (b): fixed infection rate $\alpha$. In the inset, the same dependence as in the main plot is depicted on the log-log scale.

where we equaled the total flow to the flow of the equivalent random walk system, to be able to vary the commuter ratio and the flow between the locations independently. The travel rate $\omega$ is just a parameter measuring the flow, and it is convenient for comparison of our bidirectional recurrent model with the diffusive one. From Equations (4.26),(4.27) and (4.28) it follows

$$
\omega_{1}=\frac{1+2 \varepsilon}{2} \omega \text { and } \omega_{2}=\frac{1+2 \varepsilon}{2 \varepsilon} \omega .
$$

Note that in the case of equal forward and backward rates $(\varepsilon=1), \omega$ from Equation (4.29) is not equal to $\omega$ from Equation (4.24): $\omega_{1}=\omega_{2}=3 \omega / 2$.

The expression for the velocity can be rewritten as

$$
c=\frac{2 \alpha \varepsilon \sqrt{2 D\left(2+\frac{1}{\varepsilon}\right)}}{1+2 \varepsilon+2 \alpha \varepsilon(1+2 \varepsilon)^{-1} \omega^{-1}} .
$$

The formula (4.30) represents the velocity of the SI epidemic mediated by the recurrent host movements. It depends on three important parameters: $\alpha$ - infection rate, $\omega-$ travel rate and $\varepsilon-$ ratio of commuters or travellers. In the next sections we will analyse the velocity dependence, paying special attention to limiting cases of "large" and "small" values of parameters in respect to the reference parameter value. We will also verify our analytical predictions against numerical solutions of corresponding ordinary differential equations. Detailed comparison of the results with numerical stochastic simulations and predictions for the FKPP reaction-diffusion model is postponed to Section 4.5.

\subsubsection{Dependence of the front velocity on the travel rate}

Let us consider the front velocity dependence on the travel rate. In Figure 4.5(a), the dependence $c(\omega)$ for different values of infection rate $\alpha=0.5,1,2$ and commuter ratio $\varepsilon=1$ is 

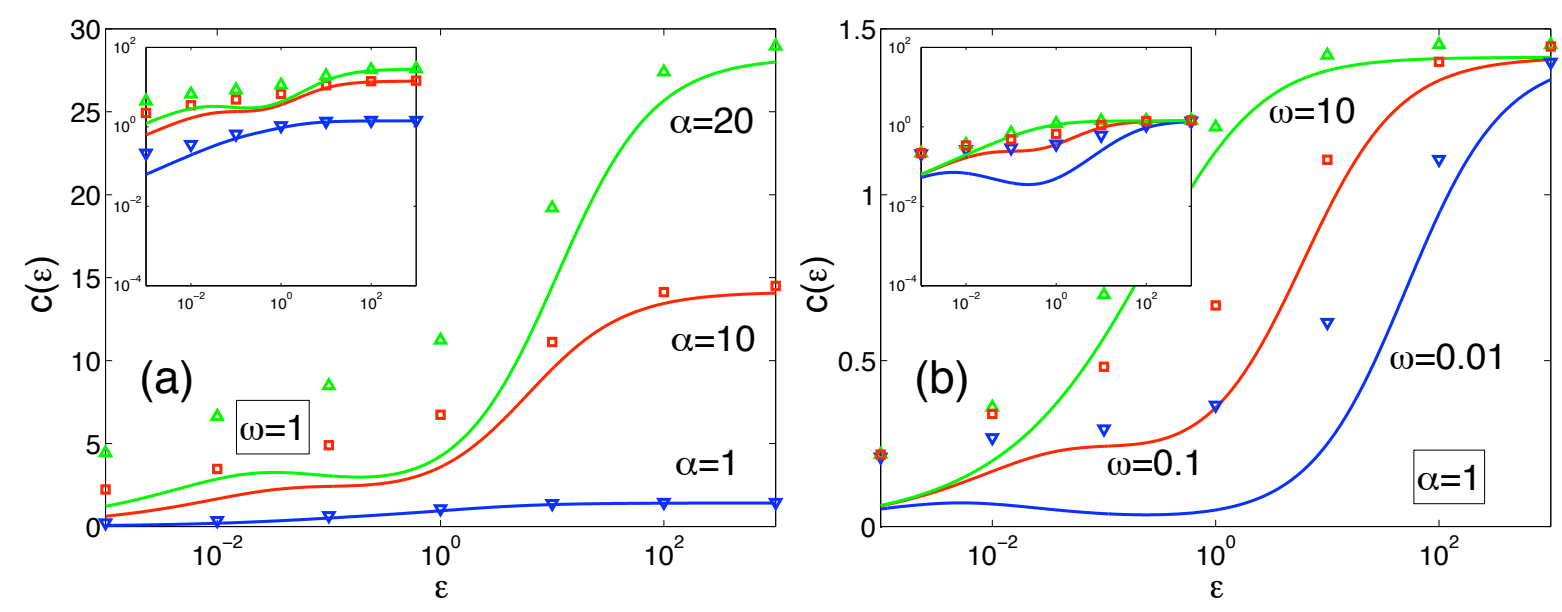

Figure 4.6: Dependence $c(\varepsilon)$ of the front velocity on the commuter ratio. Curves represent analytical results given by Equation (4.30) (with $D=1 / 2$ ). Symbols repsresent results of the numerical solution of the system of ODEs (4.20). Panel (a): fixed travel rate $\omega$. Panel (b): fixed infection rate $\alpha$. In the inset, the same dependence as in the main plot is depicted on the log-log scale.

presented. If there is no travel, i.e. $\omega=0$ then there is no epidemic wave propagation. From zero level, the velocity grows monotonously with increasing travel rate. Initially, it increases rather sharply. After $\omega \sim 1$ it saturates rapidly toward its maximal value, which is also the infection rate dependent and is given by

$$
\lim _{\omega \rightarrow \infty} c=2 \alpha \sqrt{\frac{2 D \varepsilon}{1+2 \varepsilon}} .
$$

Comparing our analytical curves with the the results of numerical solution of the system (4.20), we observe the deviation. It is nothing strange per se, because the analytical solution is the result of linearization of the continuum limit approximation of the discrete system (4.20). Actually, it is rather suprising that the velocity obtained this way is still feasible. The deviation is especially strong for large values of the infection rates and small values of the travel rate. We explain these phenomena in Section 4.4.5 and 4.5.2.

If we fix the infection rate $(\alpha=1)$ and consider the dependence $c(\omega)$ for different values of the commuter ratio $\varepsilon=10^{-2}, 10^{-1}, 10$ (Figure $4.5(\mathrm{~b})$ ), the velocity starting from zero monotonously increases again with subsequential saturation towards the $\varepsilon$-dependent high travel rate limit (4.31). At small values of the travel rates $\left(\omega \sim 10^{-2}\right)$ we can observe a non-monotonous dependence on the commuter ratio, which is more apparent from Figure 4.6(b). This prediction is however, not supported by numerical integration, as can be observed in Figure 4.5(b). For small commuter ratio values, we observe the pronounced discrepancy between numerics and analytics This will be explained in Section 4.5.

\subsubsection{Dependence on the commuter ratio}

In Figure 4.6(a), we depicted the front velocity as a function of the commuter ratio $\varepsilon$ for different infection rates $\alpha=1,10,20$ with the fixed travel rate $(\omega=1)$. If there are no 

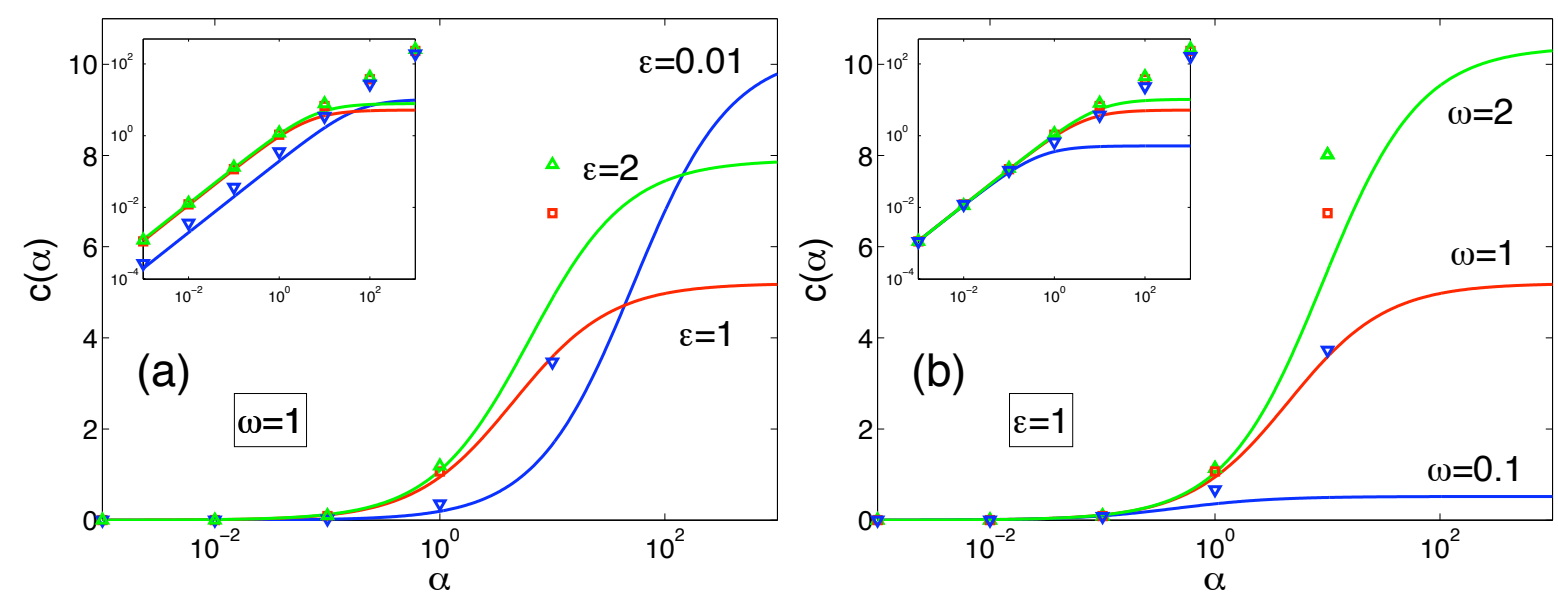

Figure 4.7: Dependence $c(\alpha)$ of the front velocity on the infection rate. Curves represent analytical results given by Equation (4.30) (with $D=1 / 2$ ). Symbols repsresent results of the numerical solution of the system of ODEs (4.20). Panel (a): fixed travel rate $\omega$. Panel (b): fixed commuter ratio $\varepsilon$. In the inset, the same dependence as in the main plot is depicted on the log-log scale.

commuters $(\varepsilon=0)$ there is also no wave propagation. For small infection rates $(\alpha=1)$, we observe the slight sigmoidal dependence on $\varepsilon$. With increasing infection rate $(\alpha=10)$ in the vicinity of $\varepsilon \sim 1$, a plateau emerges, which develops the pronounced local minimum for high infective rates. However, this analytically predicted effect is not supported by numerical solutions. The high $\varepsilon$ analytical limit depends on the infection rate as

$$
\lim _{\varepsilon \rightarrow \infty} c=2 \alpha \sqrt{D}
$$

It seems rather peculiar that we have no dependence on the travel rate $\omega$ in the limit of large fraction of individuals leaving their locations to neighbouring ones $(\varepsilon \rightarrow \infty)$. Thus, the frequency with which individuals leave their home and stay in the new locations for a long time (effectively swapping their homes), does not matter at all. On the other hand, in the scenario of high travel rates $(\omega \rightarrow \infty)$ (4.31) the commuter ratio $\varepsilon$ almost does not influence the front velocity as far as the fraction of travellers exceeds unity. Similar to the previous section, we have the strongest deviations from the analytical prediction for small $\varepsilon$ and high $\alpha$.

Let us note that the case of high travel rates $(\omega \rightarrow \infty)$ and large ratio of travellers $(\varepsilon \rightarrow \infty)$ leads to the result which can be obtained assuming the direct coupling of the locations to their neighbours (Section 4.4.7). This implies however, the implausible assumption that travellers stay at their neighbour location most of the time. Note that the velocity dependence (4.32) was obtained also in (Postnikov and Sokolov, 2007), where the direct coupling among locations without self-coupling was heuristically assumed, and no microscopic mechanism for the latter was proposed. For the detailed discussion, consult Section 4.4.7.

As can be observed for a given moderate travel rate $(\omega=1)$, the ratio of commuters $\varepsilon$ significantly influences the front velocity and thus the $\varepsilon$-dependence cannot be neglected. The zone of strong increase is weakly dependent on $\alpha$ and is situated between $\varepsilon \sim 1$ and $\varepsilon \sim 10^{2}$ 
(4.6(a)). As one can see from Figure 4.6, the deviation from the theoretical prediction is the least for moderate values of the infection rate $\alpha$.

For the fixed infectiousness $(\alpha=1)$, the dependence on the commuter ratio for different travel rates $\omega=10^{-3}, 10^{-1}$ and 10 is depicted in Figure4.6(b). The usual sigmoidal curve can be observed only for high travel rates $(\omega=10)$. For intermediate travel rates $\left(\omega=10^{-1}\right)$, we observe the plateau at $\varepsilon \sim 10^{-1}$ and for smaller travel rates $\left(\omega=10^{-2}\right)$, we observe the pronounced local minimum at $\varepsilon \sim 10^{-1}$. However, these analytical predictions are not confirmed by numerical results. The zone of sharp increase of the sigmoidal curve is not universal for all $\omega$ values.

\subsubsection{Dependence on the infection rate}

Finally, let us consider the dependence of the front velocity on the infection rate $\alpha$. It is usually tightly connected to the pathogens properties and cannot be modified as easy as all other parameters. However, the infectiousness of diseases can span a range from ${ }^{5} R_{0}=3-4$ for influenza, to $R_{0}=16-18$ for whooping cough (Keeling and Rohani, 2007). Besides, we should not forget that all rate quantities are relative in respect to the reference one. Thus the high infectiousness can mean the very low host mobility.

As it is seen in Figure 4.7(a),(b), the familiar from the previous sections sigmoidal analytical curve starting from zero (for zero infectiousness there is no epidemic) is reproduced for both cases of fixed travel rate $\left(\omega=10^{-1}, 1,2\right)$ and fixed commuter ratio $\left(\varepsilon=10^{-2}, 1,2\right)$. The analytical high infection rate limit depends on both the commuter ratio and the travel rate as

$$
\lim _{\alpha \rightarrow \infty} c=\sqrt{2 D\left(2+\frac{1}{\varepsilon}\right)}(1+2 \varepsilon) \omega .
$$

However, this is a delusion. Numerical results witness the surprising, ever-growing $\alpha$-dependence of the velocity. The increasing dependence of the velocity on the reaction rate may be an artifact of the continuum approximation, i.e. a continuous density of individuals implying an infinitive number of agents. The flow of agents is proportional to the number of them times the travel rate. Thus, in the system with an infinite number of agents, there is no restriction on the velocity from the movements of single agents. The reaction rate is the only restriction. This does not hold any more for a discrete system with a finite number of agents where the upper bound on the velocity, namely, $c \sim N \omega$ clearly has to exist. In the case of fixed travel rates (4.7(b)) a non-monotonous theoretical dependence of the velocity on the commuter ratio for high infection rates, already seen in the figure 4.6(a), is depicted. However, the non-monotonous behavior is not recovered in the numerics. The zone of strong increase is $\varepsilon$ - and $\omega$-independent. Numerical solutions of the mean-field model can be described by the analytical prediction in the range of small to intermediate infection rates.

Note that the unbounded velocity dependence on the infection rate obtained by numerical integration can be observed in the ordinary FKPP case as well. In Figure 4.8, analytical

\footnotetext{
${ }^{5}$ The basic reproduction number in the case of the SIS or the SIR epidemics reads $R_{0}=\alpha / \beta$. Refer also to Chapter 2.
} 


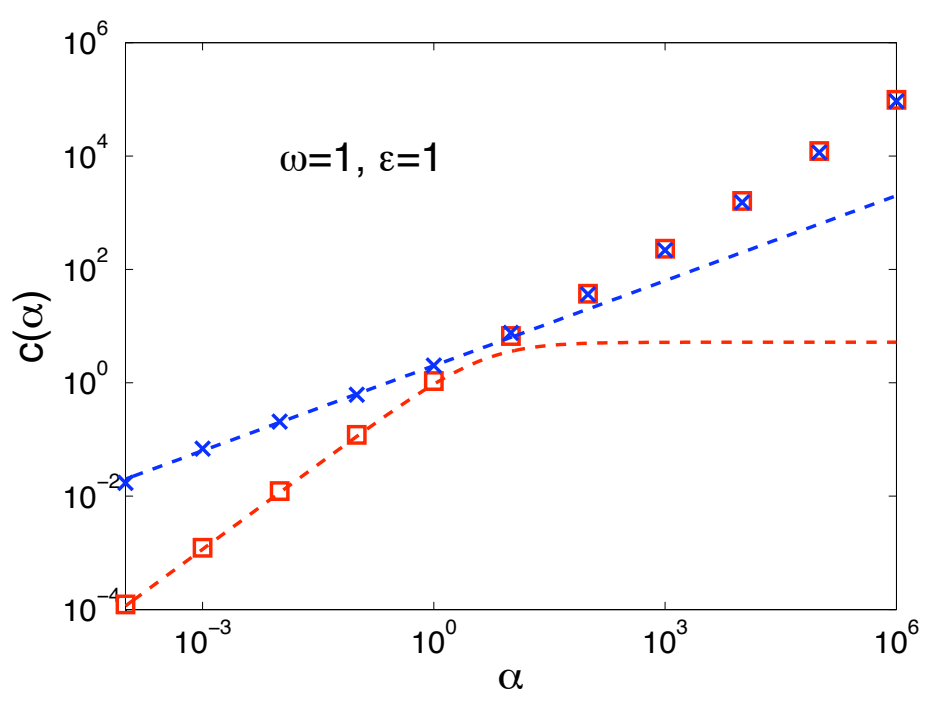

Figure 4.8: Dependence $c(\alpha)$ of the front velocity on the reaction rate for the random walk travel (blue line) and bidirectional recurrent travel (red line) obtained as the result of numerical solution of the system of ODEs. While the high reaction rate limits coincide for both models and deviate from the analytical predictions (dashed lines) given by (4.25) and (4.30), the small reaction rate asymptotics is well described by analytical predictions for both models.

velocity predictions for epidemics, mediated by recurrent host movements and by random walk, are compared. The analytics yields a perfect agreement with numerical integration in the range of small to intermediate infection rates in the FKPP case as well.

Various limit cases as well as asymptotic Taylor expansions of the formula (4.23) in large or small parameter ranges are summarized in Tables 4.1 and 4.4.5. Misleading results not confirmed by numerical integration are marked in red. Besides the above-discussed features the following can be noticed. For small values of the travel rate, the asymptotic predicts a linear dependence on the travel rate. Rather surprisingly, this is also true for a system with a finite agents number but is not true in the mean-field case (cf. Section 4.5) as revealed by the numerical integration. For high travel rates, it is only the additional term proportional to $\omega^{-1}$ to the limit velocity value in which the travel rate comes into play. For high commuter ratio, we also have the proportionality to $\varepsilon^{-1}$ only in the correction to the high commuter ratio limit. Thus, both the travel rate and the commuter ratio in this sense behave very similarly in the regime of large parameter values.

\subsubsection{Front shape}

The shape of the epidemic front and the front velocity in the FKPP model stay in immediate relation to each other (Murray, 1993). We can assume that in the co-moving frame, the leading front of the wave is also given by the decaying exponential $u(z)=u_{0} e^{-s z}$. After linearization of (4.18), we obtain the relation between the decay coefficient of the exponential $s$ and the front velocity

$$
c=\mathcal{D} s+\frac{\alpha}{s} .
$$

Having in mind the minimal velocity (4.25), we obtain the decay coefficient

$$
s=\sqrt{\frac{\alpha}{\mathcal{D}}}
$$




\begin{tabular}{|c|c|c|c|}
\hline & $\varepsilon \sim \mathcal{O}(\alpha)$ & $\omega \sim \mathcal{O}(\alpha)$ \\
\hline$\omega \rightarrow 0$ & 0 & $\varepsilon \rightarrow 0$ & 0 \\
\hline $0<\omega \ll \alpha$ & $\sqrt{2 D\left(1+\frac{1}{\varepsilon}\right)}(1+2 \varepsilon) \omega+\mathcal{O}\left(\omega^{2}\right)$ & $0<\varepsilon \ll \alpha$ & $2 \sqrt{2 D \varepsilon}+\mathcal{O}\left(\varepsilon^{\frac{3}{2}}\right)$ \\
\hline$\alpha \ll \omega<\infty$ & $\frac{2 \alpha \sqrt{2 D\left(2+\frac{1}{\varepsilon}\right)} \varepsilon-\frac{4 \alpha^{2} \sqrt{2 D\left(2+\frac{2}{\varepsilon}\right)}}{(1+2 \varepsilon)^{3}} \frac{\varepsilon^{2}}{\omega}+\mathcal{O}\left(\frac{1}{\omega^{2}}\right)}{1+2 \varepsilon} \varepsilon \ll \varepsilon<\infty$ & $2 \alpha \sqrt{2 D}-\frac{\alpha \sqrt{D}(2 \alpha+\omega)}{2 \varepsilon \omega}+\mathcal{O}\left(\frac{1}{\varepsilon^{2}}\right)$ \\
\hline$\omega \rightarrow \infty$ & $\frac{2 \alpha \sqrt{2 D\left(2+\frac{1}{\varepsilon}\right)}}{1+2 \varepsilon} \varepsilon$ & $\varepsilon \rightarrow \infty$ & $2 \alpha \sqrt{2 D}$ \\
\hline
\end{tabular}

Table 4.1: Asymptotic expansions of Equation (4.30) for the extreme values of the travel rate $\omega$ and the commuter ratio $\varepsilon$. The infection rate $\alpha$ was taken as the reference parameter. Results not confirmed by numerical integration are marked in red.

\begin{tabular}{|c|c|}
\hline$\alpha \rightarrow 0$ & 0 \\
\hline $0<\alpha \ll \omega, \varepsilon$ & $\alpha \frac{2 \varepsilon \sqrt{2 D\left(2+\frac{1}{\varepsilon}\right)}}{1+2 \varepsilon}+\mathcal{O}\left(\alpha^{2}\right)$ \\
\hline$\omega, \varepsilon \ll \alpha<\infty$ & $\sqrt{2 D\left(2+\frac{1}{\varepsilon}\right)}(1+2 \varepsilon) \omega-\frac{\sqrt{2 D\left(2+\frac{1}{\varepsilon}\right)}(1+2 \varepsilon)^{3}}{\varepsilon} \frac{\omega^{2}}{\alpha}+\mathcal{O}\left(\frac{1}{\alpha^{2}}\right)$ \\
\hline$\alpha \rightarrow \infty$ & $\sqrt{2 D\left(2+\frac{1}{\varepsilon}\right)}(1+2 \varepsilon) \omega$ \\
\hline
\end{tabular}

Table 4.2: Asymptotic expansions of the general formula (4.30) for extreme values of the infection rate $\alpha$. Expressions in red are not confirmed by numerical solution of the original system of ODEs (4.20).

We can proceed analogously in the case of bidirectional movements. However, this approach reveals a surprise. Namely, calculations similar to those performed above for the FKKP model predict the non-monotonous dependence of the decay coefficient on the travel rates

$$
s=-\sqrt{\frac{2}{D}\left(1+\frac{\omega_{2}}{\omega_{1}}\right)} \frac{\alpha+\omega_{2}+2 \omega_{1}}{\alpha-\omega_{2}-2 \omega_{1}} .
$$

However, from Figures 4.9 and 4.10, in which front shapes for the SIR epidemics are depicted, we observe, that the decay exponent $s$ decreases monotonously with the travel rate. This may indicate that the asymptotic is not exponential on long scales, because this ansatz fails to reproduce the real dependence of the front, or this ansatz is not valid because $u$ and $v$, taken alone, does not fulfill it. 


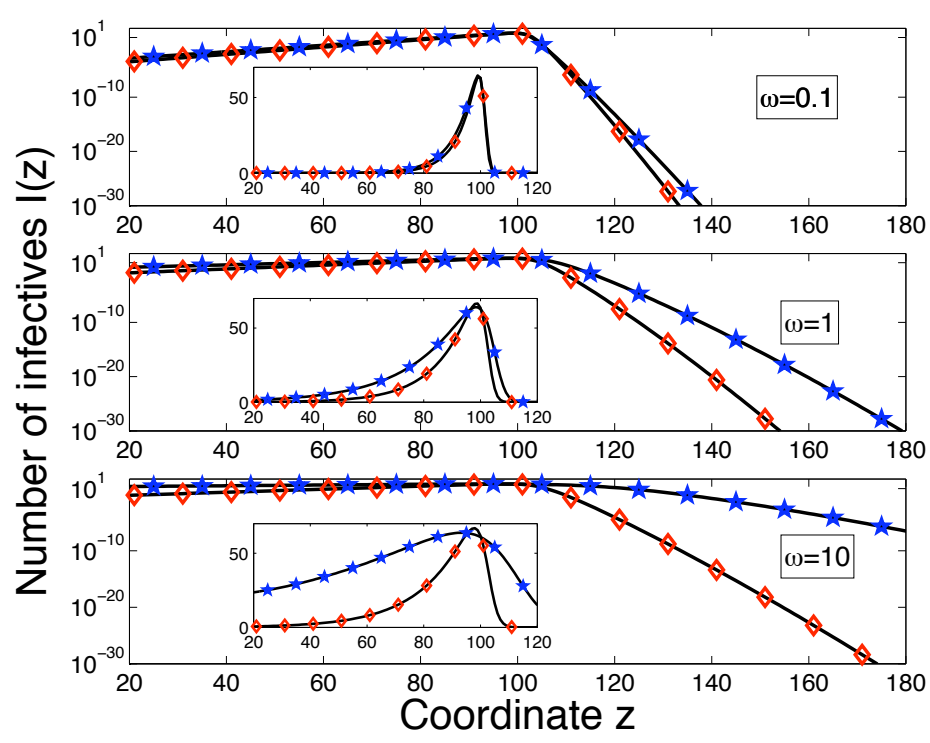

Figure 4.9: Front shape of the SIR epidemics for the random walk (blue stars) and the recurrent (red diamonds) movement pattern of the hosts for three values of travel rates $\omega=0.1,1$ and 10 . Other parameters: $\alpha=1, \beta=0.1$. We considered 200 locations with population size $N=100$. Insets shows the sames curves in linear scale.

Note that in the limit of high travel rates we obtain from Equation (4.34)

$$
\lim _{\varepsilon \rightarrow \infty} s=\frac{1}{d} \sqrt{\frac{1+2 \varepsilon}{\varepsilon}}
$$

which coincides with the result $(4.46)^{6}$ found in Section 4.4.7.

In Figure 4.9, the wave front for the case of the SIR epidemic is compared with the wave front for the FKPP model. While the fronts seems similar for small travel rates, the difference becomes more apparent with an increasing travel rate. Here again, as in Section 4.4.2, we have introduced the effective travel rate and the commuting ratio for the bidirectional model in order to compare both models. In Figure 4.10, front shape of the model with recurrent movements for different values of the commuter ratio $(\varepsilon=0.1,1,10)$ is compared with the direct coupling model and the incomplete SIO direct coupling model.

\subsubsection{Epidemics with direct coupling}

Now we consider the direct coupling model (see Chapter 2). As it was shown in Section 4.2 , for a general graph topology, the directly coupled model arises from the model with recurrent host movements in the limit of large travel rates. In the present section, we start from the heuristic direct coupled model, obtain the corresponding continuum limit equations, and analyse its characteristics, such as the wave front velocity and the slope of the wave front using our knowledge of the relation between coupling strengths and actual travel rates of individuals obtained in Section 4.2.

We start with the following scheme of the chemical reactions describing the SI epidemic in one particular location $n$

\footnotetext{
${ }^{6}$ To be specific, (4.46) gives the slope for the full SI model. As will be discussed in Section 4.4.7, the slope of the SI0 wave (4.35), which we are discussing now differs by a square root of two from (4.46).
} 


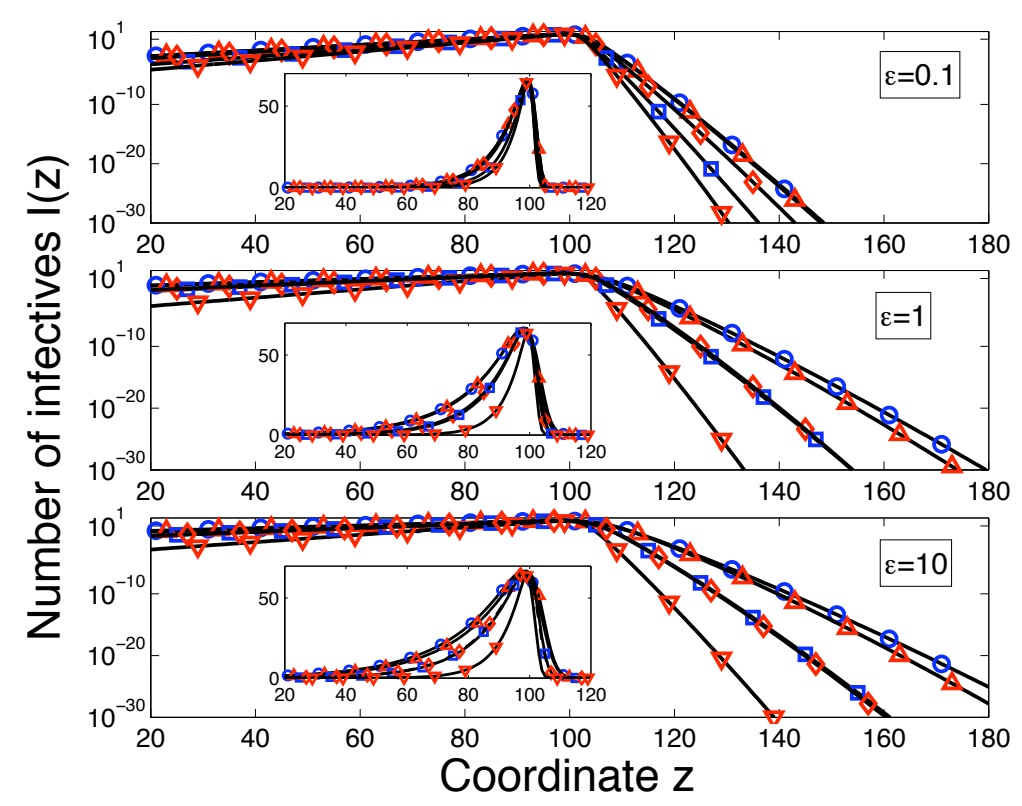

$$
\begin{array}{rll}
I_{n}+S_{n} & \stackrel{\alpha}{\rightarrow} & 2 I_{n} \\
I_{m}+S_{n} & \stackrel{\alpha \epsilon_{n m}}{\rightarrow} & I_{m}+I_{n} \\
I_{n} & \stackrel{\beta}{\rightarrow} & S_{n}
\end{array}
$$

Figure 4.10: Front shape for SIR epidemics for different values of commuter ratio $\varepsilon=$ $0.1,1$ and 10 calculated numerically by solving the set of corresponding ODEs. Red symbols denote results for the recurrent model for $\omega=0.1$ $(\nabla), \omega=1(\diamond)$ and $\omega=$ $10(\triangle)$. Blue symbols denotes results for the direct coupled model with neglected second neighbour coupling (4.45) $(\square)$ and the full direct coupled model (4.37)(o). Infective rate $\alpha=1$, recovery rate $\beta=0.1,200$ locations with population size $N=100$. Insets shows the sames curves in usual scale.

where we have phenomenologically introduced the coupling strength $\epsilon_{n m}$, characterizing the effective ever-presence of individuals due to their frequent travel. Thus, we can extend the usual SIS kinetic equation for one particular location $n$ to

$$
\partial_{t} I_{n}=\frac{\alpha}{N}\left(N-I_{n}\right) \times\left(\sum_{m} \epsilon_{n m} I_{m}\right)-\beta I_{n} .
$$

As previously mentioned in Chapter 2, such a description is rather straightforward and is frequently used in epidemiological models (Rushton and Mautner, 1955; Daley and Gani, 1999; Camitz and Liljeros, 2006; Viboud et al., 2006). Phenomenologically introduced coupling strengths $\epsilon_{n m}$ are unknown although they may be in principle derived a posteriori from epidemiological data using some heuristic assumptions and by fitting model parameters to the actual course of a past epidemic. However, as we showed previously, the coupling strengths can be derived immediately from the assessable quantities such as travel rates, given they are sufficiently high, using Equations (4.14) and (4.13). If we consider the homogeneous chain of locations once again, i.e. all locations are of the same size (see Figure 4.4) labelled by the index $n=1, \ldots, N$ and use the ratio of commuters $\varepsilon=\omega_{1} / \omega_{2}$ (cf. Section 4.4.2), then we 
obtain for the coupling strengths:

$$
\epsilon_{n m}=\frac{\delta_{n m}+\left(1-\delta_{n m}\right) \varepsilon}{(1+2 \varepsilon)^{2}} .
$$

We assume equal coupling strengths between the next-neighbors $\epsilon_{n m}=\epsilon \delta_{n \pm 1, m}$ and selfcoupling $\epsilon_{n n}=\epsilon_{0}$. Then we can write

$$
\begin{aligned}
\frac{d}{d t} I_{n}= & \frac{\alpha}{N} S_{n}\left[p_{n-2}^{n-1} p_{n}^{n-1} I_{n-2}+\left(p_{n-1}^{n-1} p_{n}^{n-1}+p_{n-1}^{n} p_{n}^{n}\right) I_{n-1}\right. \\
& +\left(p_{n}^{n-1} p_{n}^{n-1}+p_{n}^{n} p_{n}^{n}+p_{n}^{n+1} p_{n}^{n+1}\right) I_{n} \\
& \left.+p_{n+2}^{n+1} p_{n}^{n+1} I_{n+2}+\left(p_{n+1}^{n+1} p_{n}^{n+1}+p_{n+1}^{n} p_{n}^{n}\right) I_{n+1}\right]-\beta I_{n}
\end{aligned}
$$

where from (4.36) and (4.14) we obtain

$$
\frac{d}{d t} I_{n}=\frac{\alpha}{N(1+2 \varepsilon)^{2}} S_{n}\left[\varepsilon^{2}\left(I_{n-2}+I_{n+2}\right)+2 \varepsilon\left(I_{n-1}+I_{n+1}\right)+\left(1+2 \varepsilon^{2}\right) I_{n}\right]-\beta I_{n}
$$

If dynamics in the neighbor locations is similar enough, i.e. $\frac{I_{n-1}-I_{n}}{I_{n}} \ll 1$, we can make use of the Taylor expansion

$$
I_{n \pm 1}=I(x \pm d) \approx I(x) \pm d \nabla I+\frac{d^{2}}{2} \Delta I
$$

and similarly for $I_{n \pm 2}$. Inserting the Taylor approximation into Equation (4.38), we obtain

$$
\partial_{t} I=\frac{\alpha}{N(1+2 \varepsilon)^{2}}(N-I)\left[\left(4 \varepsilon^{2}+4 \varepsilon+1\right) I+\left(4 \varepsilon^{2}+2 \varepsilon\right) d^{2} \Delta I\right]-\beta I .
$$

Thus, for the density of infectives $u=I / N$ in one location we have

$$
\partial_{t} u=\alpha(1-u) \tilde{D} \Delta u+\alpha\left(u^{\star}-u\right) u
$$

where $u^{\star}=1-\beta / \alpha$ and

$$
\tilde{D}=\frac{2 \varepsilon d^{2}}{1+2 \varepsilon}
$$

We should note here that the similar continuum limit equations were obtained phenomenologically in more general form in (Lopez et al., 1999) and recently in (Postnikov and Sokolov, 2007). However, no connection of coupling strengths in the continuum limit to microscopical travel mechanism have been considered so far. This leads to the effective coupling only to the next neighbors (cf. Section 4.4.7.1) although Equation (4.37) witness coupling to the second next neighbors as well.

Equation (4.39) cannot be solved analytically. If however, we consider the SIR infectious dynamics, then one can show that the first integral of the ordinary differential equations obtained by using the travelling-wave ansatz exists. For example, in (Postnikov and Sokolov, 2007), such continuum version of SIR epidemics with phenomenologically direct coupling was considered, given by equations

$$
\begin{aligned}
\partial_{t} u & =\alpha(1-u-w)(u+\tilde{D} \Delta I)-\beta u \\
\partial_{t} w & =\beta u,
\end{aligned}
$$


where $u$ is now the density of infectives, $v$ is the density of susceptibles and $w$ is the density of recovered individuals from one particular location. In the comoving frame the invariant for the continuum version of the directly coupled SIR epidemic reads (Naether et al., 2008)

$$
\ln v+\frac{\alpha c}{\beta} w+\frac{\alpha \tilde{D} c}{\beta} w^{\prime \prime}=0
$$

leading to the solutions of the equations $4.41,4.42$ in a closed form

$$
\begin{aligned}
& v(z)=\frac{1}{2}\left[1+\tanh \left(\frac{c}{8 \alpha \tilde{D}}\left(z-z_{0}\right)\right)\right] \\
& u(z)=1-v(z)+\frac{\beta}{\alpha} \ln v(z)
\end{aligned}
$$

with the density of recovered individuals $w=1-u-v$.

\subsubsection{Front velocity}

Inserting the travelling wave ansatz $u(x, t)=U(x-c t)$ into Equation (4.39), we obtain the ordinary differential equation

$$
-c U^{\prime}=\alpha(1-U) \tilde{D} U^{\prime \prime}+\alpha\left(U^{\star}-U\right) U
$$

We can reduce this second order differential equation to the system of first order ones:

$$
\left\{\begin{array}{c}
U^{\prime}=V \\
\alpha(1-U) \tilde{D} V^{\prime}=-\alpha\left(U^{\star}-U\right) U-c V .
\end{array}\right.
$$

After the change of variable $z \rightarrow \zeta$, such that $\frac{d V}{d \zeta}=-\alpha(1-U) \tilde{D} \frac{d V}{d z}$, to get rid of singularity, the system of equations reads

$$
\left\{\begin{array}{c}
U^{\prime}=\alpha(1-U) \tilde{D} V \\
V^{\prime}=-\alpha\left(U^{\star}-U\right) U-c V
\end{array} .\right.
$$

As expected, fixed points of the system are $(U, V)=(0,0)$ and $(1,0)$. To fulfill boundary conditions: $u \rightarrow 0$ if $z \rightarrow \infty$ and $u \rightarrow 1$ if $z \rightarrow-\infty$, a trajectory (heteroclinic orbit) should start in the unstable fixed point $(1,0)$ and go without oscillation $(u \geq 0)$ straightly to the stable one $(0,0)$. To determine the conditions under which this is possible, we can perform the linear stability analysis for the zero-fixpoint $(0,0)$. For this, we have to solve the eigenvalue problem $(A-I \lambda) X=0$, with the determinant

$$
\operatorname{det}(A-I \lambda)=\left|\begin{array}{cc}
-\lambda & \alpha \tilde{D} \\
-\alpha U^{\star} & -c-\lambda
\end{array}\right| .
$$

Solutions of the secular equation are

$$
\lambda_{ \pm}=-\frac{c}{2} \pm \frac{1}{2} \sqrt{c^{2}-4 \alpha^{2} \tilde{D} U^{\star}} .
$$


Thus, for the zero fixed point to be stable ${ }^{7}$ we should require

$$
c \geq 2 \alpha \sqrt{\tilde{D} U^{\star}}
$$

For steep enough initial conditions, the last inequality becomes a strict equation (Murray, 1993). The corresponding velocity is called the minimal velocity and it is selected from all other possible ones (Kessler et al., 1998). Note that from Equation (4.40) follows for the velocity

$$
c=2 \alpha d \sqrt{\frac{2 \varepsilon}{1+2 \varepsilon} U^{\star}} .
$$

If we compare the last result with the high effective travel rate limit of our full bidirectionally coupled model (4.31), i.e. $c_{\mathrm{bd}}=2 \alpha d \sqrt{\varepsilon /(1+2 \varepsilon)}$, we observe that it differs from (4.44) by a factor of $\sqrt{2}$. That is an artifact of the SI0-model, where only infectives were assumed to travel. Indeed, for the SI0-model

$$
\frac{d}{d t} I_{n}=\frac{\alpha}{N} S_{n}\left(p_{n-1}^{n} I_{n-1}+p_{n}^{n} I_{n}+p_{n+1}^{n} I_{n+1}\right)-\beta I_{n}
$$

because the probability for a susceptible to be at home is unity and further

$$
\frac{d}{d t} I_{n}=\frac{\alpha}{N(1+2 \varepsilon)}\left[I_{n}+\varepsilon\left(I_{n-1}+I_{n+1}\right)\right]-\beta I_{n}
$$

Thus, finally

$$
\partial_{t} u=\alpha(1-u) \tilde{D}_{\mathrm{SI} 0} \Delta I+\alpha\left(u^{\star}-u\right)
$$

with $\tilde{D}_{\mathrm{SI} 0}=\frac{\varepsilon d^{2}}{1+2 \varepsilon}$ leading as expected to the velocity (4.31). Thus, even in the high commuter ratio limit, the prediction from (Postnikov and Sokolov, 2007) is valid only for infectives travelling and immobile susceptibles, leading to the nearest neighbor coupling instead of the considered above the second nearest neighbor coupling.

\subsubsection{Front shape}

If we consider the front shape $U(z)$ on long distances $(z \rightarrow \infty)$, we see from (4.39) that it is determed mostly by the exponential growth term. Thus we can approximate it by the exponential $U(z) \sim e^{-s z}$ (Murray, 1993). Inserting this ansatz into (4.39) and allowing terms only of the first order in $U$, we have

$$
\alpha \tilde{D} s^{2}-c s+\alpha U^{\star}=0 .
$$

Thus, for the wave front slope we obtain

$$
s=\frac{c}{2 \alpha \tilde{D}}\left(1 \pm \sqrt{1-\frac{4 \alpha^{2} \tilde{D} U^{\star}}{c^{2}}}\right) .
$$

\footnotetext{
${ }^{7}$ Note, that $c>0$.
} 
One can show that, if the initial conditions are steep enough, the front moves with the minimal velocity $c=2 \alpha \sqrt{\tilde{D} U^{\star}}$ (4.43), and hence, it follows for the slope exponent

$$
s=\sqrt{\frac{U^{\star}}{\tilde{D}}}
$$

or in terms of the commuter ratio $\varepsilon$ using (4.40)

$$
s=\sqrt{\frac{(1+2 \varepsilon) U^{\star}}{2 \varepsilon d^{2}}} .
$$

This dependence of the wave front slope on the commuter ratio is illustrated in Figure 4.11. If there are a few commuters $(\varepsilon \ll 1)$, we obtain very large values for the slope $s$ (almost vertical slope) which correspond to an almost very slow epidemic spread equivalent to a hopping of the epidemic among locations. If there are many commuters $(\varepsilon \gg 1)$, the slope of the wave front is bounded from below by $\lim _{\varepsilon \rightarrow \infty} s=\sqrt{U^{\star}} / d$. This is also illustrated in Figure 4.10, in the case of SIR epidemics ${ }^{8}$. Another noticeable property of the expression (4.46) is the independence of the slope on the infection rate in the SI-case $(\beta=0)$ or slight dependence in the SIS-case contrary to the analogous result for the ordinary FKPP model (4.33)

$$
s_{\mathrm{FKPP}}=\sqrt{\frac{\alpha U^{\star}}{\omega D}} .
$$

Thus, for the front shape of the directl coupling model, the reaction rate plays almost no role. However, in the limit of high travel rates, the FKPP wave front slope is almost zero $(s \rightarrow 0)$ contrary to the bidirectionally coupled model which has a slope bounded from below. Front shape stays in immediate relation to the velocity. In Section 4.5, we will discuss effects of finite number of agents on the front-speed and the ansatz due to Brunet and Derrida (Brunet and Derrida, 1997). Their prominent result states that the deviation from the analytical expression for the minimal velocity scales as $\delta c \sim 1 /(\ln N)^{2}$ with the number of agents pro site $N$. This effect can also be found in our case. The velocity arising in different coupling schemes may vary by a prefactor in this relation. Furthermore, in the light of the artifact of continuous description discussed in Section 4.4.5 leading to the unbounded velocity dependence on the infection rate, it is worth to numerically check the dependence of the front shape on the infection rate.

Note that in the case of SIR epidemics, the result (4.46) for the leading front of the density of infectives still holds. In the SIR epidemics, we have the rear front as well. One can assume $u(z) \sim e^{r z}$ for a region far away from the bulk of infectives, where the number of infectives is very small and the number of recovered is approximately unity $(w \approx 1)$. Then the linearized version of equations (4.41) reads

$$
\partial_{t} u \approx-\beta u \text {. }
$$

Thus, for the coefficient in the exponential for the rear front we have

$$
r=\frac{\beta}{2 \alpha d \sqrt{1-\beta / \alpha}} \sqrt{\frac{1+2 \varepsilon}{2 \varepsilon}},
$$

\footnotetext{
${ }^{8}$ The leading front in the SIR model is essentially the same as in the SIS epidemics considered so far.
} 


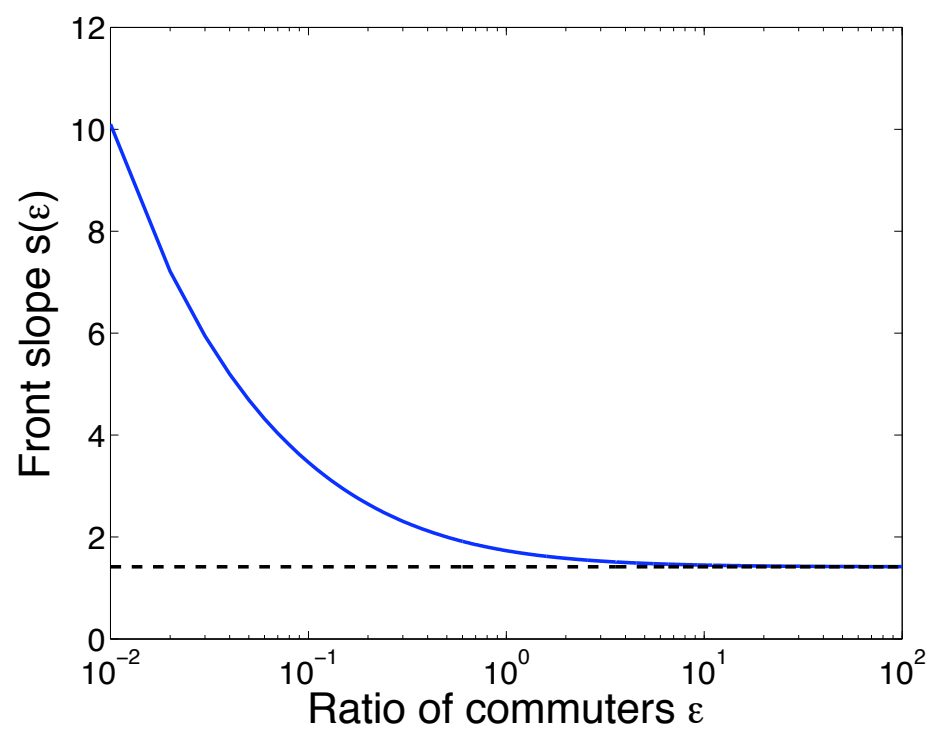

Figure 4.11: Dependence of the slope decay exponent $s(\varepsilon)$ of the wave front on the ratio of commuters $\varepsilon$ in the direct coupling case, Equation (4.46). The SI epidemic was considered $(\beta=$ $0)$.

leading to the flattening of the rear front with the increasing commuter ratio, as can be observed in Figure 4.10 where numerically calculated fronts for different models and different commuter ratios $\varepsilon$ and infection rates $\alpha$ are compared. Note that for both rear and leading fronts, the dependence on the commuter ratio is similar (cf. (4.46) and (4.47)). The result (4.47) is in accord with (Postnikov and Sokolov, 2007), in the case of high commuter ratio and no self-coupling if we recall the discussion from Section 4.4.7.1. Note also that in (Postnikov and Sokolov, 2007), an attempt was undertaken to explain the Kendall wave shape with a long rear front, which can not be accounted for by the common reaction-diffusion approach. We see that our approach can deliver even more variability in the front shapes, depending on the parameters in particular on the commuter ratio and travel rate.

We can conclude that the model of infectious spread with recurrent movements can be reduced to the direct coupling model only in the limit of high travel rates. Phenomenologically introduced coupling strengths can be related to individual travel rates. The continuum limit equations are structurally different from the FKPP case. The epidemic wave front shape looks very different from its counterpart in the reaction-diffusion framework.

\subsection{Numerics and stochastic}

The epidemic model due to recurrent host movements on a one-dimensional lattice, formulated in Section 4.4.1, can be stochastically simulated ${ }^{9}$ on a computer. In the present section the results of extensive numerical simulations are presented and new insights beyond the mean filed picture are analysed and discussed.

\footnotetext{
${ }^{9}$ The essential part of the software for numerical simulations was provided by Benjamin Schwenker whom I express my gratitude at this place. Details of the applied numerical algorithms can be found in his diploma thesis (Schwenker, 2008)
} 


\subsubsection{Brunet-Derrida scaling}

In Figure 4.12, the dependence $c(\omega)$ of the front velocity obtained from numerical simulations on the travel rate for different epidemiological models (SI0, SI and SIS) is presented. Without loss of generality, we took equal forward and backward travel rates $\omega_{1}=\omega_{2}=\omega$. For comparison, the dependence of the front velocity on the travel rate for the ordinary FKPP case is depicted as well. We observe a good agreement with our theoretical prediction. Note also that standard deviations of the velocity for different realisations denoted by error bars are very small and similar in the FKPP and recurrent case. However, the velocity obtained from the stochastic simulation for SI0 model is slightly lower, than the mean field prediction. This is no surprise, because, in the ordinary FKKP case with a random walk travel pattern, the effect of discreteness already leads to the attenuation of the wave. One can incorporate fluctuations using field theoretical methods and obtain the stochastic differential equation for the FKPP case (Pechenik and Levine, 1999)

$$
d u=\alpha u(1-u) d t+D \Delta u d t+\frac{\sigma}{\sqrt{N}} \sqrt{u(1-u)} d W(t) .
$$

The analysis of this equation shows the deviation of the front velocity from the continuum limit prediction scales with the average number of agents per site $N$ as

$$
\delta c \sim \frac{1}{(\ln N)^{2}} .
$$

The more insightful explanation of this phenomena is due to Brunet and Derida (Brunet and Derrida, 1997), and even earlier due to Bramson (Bramson, 1983). Still adhering to the meanfield model, they assumed, that the reaction cannot take place if th concentration of species is below some cut-off value $\varepsilon \sim N^{-1}$. Diffusion was however, allowed. They showed that the difference between the mean-field velocity prediction and that of the model with the cutoff is given by (4.48). This result was obtained by many different methods, although the prefactor recovering the equality in (4.48) is not unique (Panja, 2004). In Figure 4.13, the dependence of the front velocity on the number of agents per site for an SI epidemic is presented in the case of recurrent host movements. A very good agreement with the scaling expression (4.48) is observed. One can hope to apply the same cutoff approach to our model with bidirectional travel, what is a non-trivial task in our case. One could try e.g. with the following ansatz (for the SI0 model)

$$
\begin{aligned}
\partial_{t} u & =\alpha\left[1-u \vartheta_{\varepsilon}(u)-v \vartheta_{\varepsilon}(\varepsilon)\right]\left[u \vartheta_{\varepsilon}(\varepsilon)+v \vartheta_{\varepsilon}(\varepsilon)+D \Delta v\right)+\omega_{2} v-2 \omega_{1} u \\
\partial_{t} v & =2 \omega_{1} u-\omega_{2} v .
\end{aligned}
$$

Since we want the effective diffusion or supply with infectives to be sustained, we cannot introduce a cutoff before the Laplacian. Thus, we take in account fluctuations in the reactions within the population but not in the reactions where contacts with neighbours take place.

One can further investigate the temporal relaxation properties of the velocity towards its analytical mean-field prediction and the role of initial conditions à la (Kessler et al., 1998). Their results are based on consideration of the FKPP model as a limit case of the real GinsburgLandau equation. 


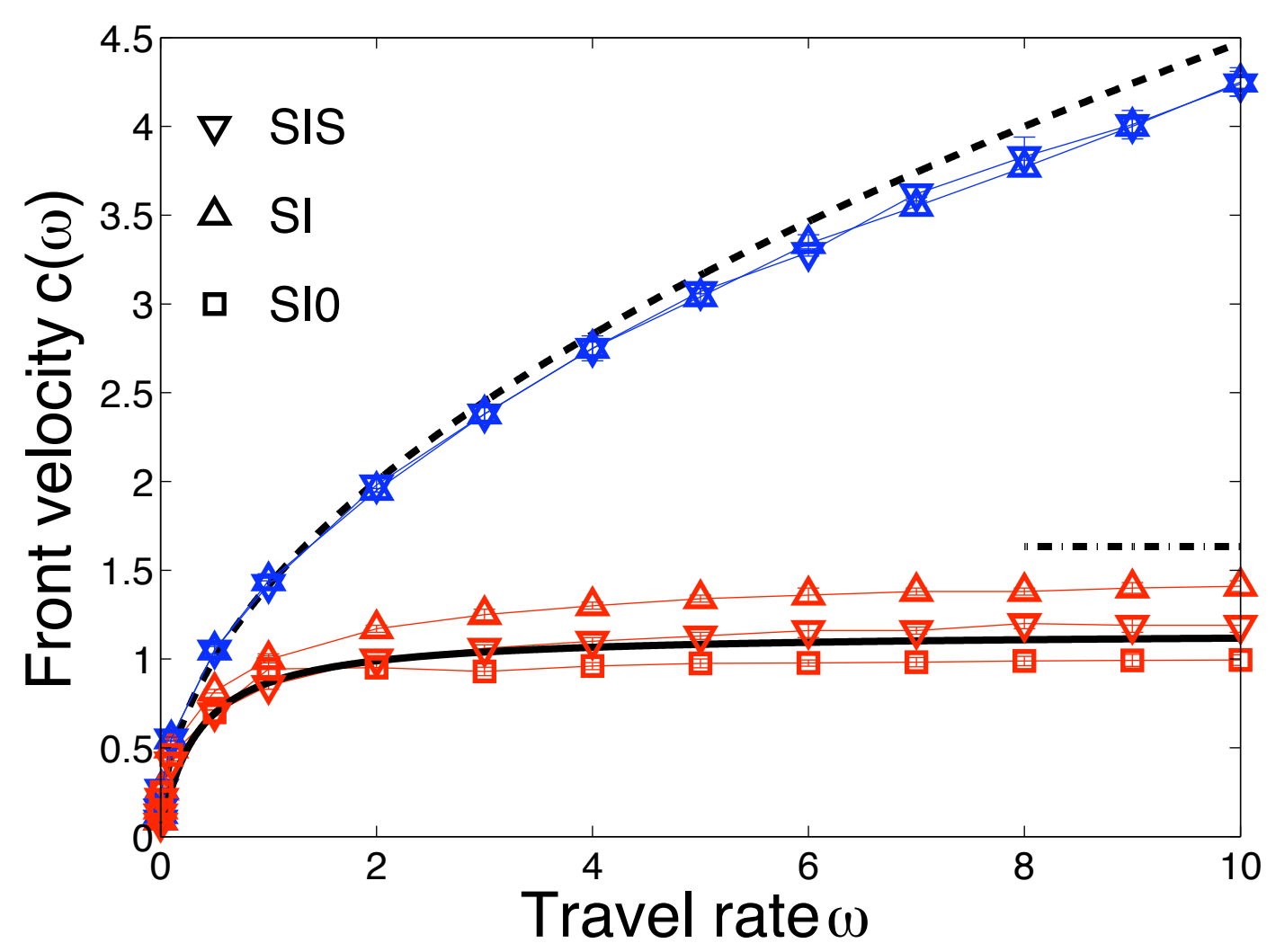

Figure 4.12: Dependence $c(\omega)$ of the epidemic front velocity on the travel rate. Symbols represent results of the agent-based stochastic simulations of the FKPP SI model (blue ones) and to the SI0 model with recurrent travel (red ones). Solid and dashed lines denote analytical predictions for the FKKP SI (4.25) and the bidirectional SI0 (4.24) models respectively. The dash-dotted line denotes the high commuter ratio limit of the velocity of the directly coupled SI model given by (4.44), or equivalently, the high travel rate limit of the recurrent SI model. The number of agents pro site, $N=10^{4}$ and recovery rate for the SIS model, $\beta=0.1$.

\subsubsection{Behavior of the front velocity for small travel rates}

Figure 4.14 presents a surprising result in the behavior of the front velocity for very low travel rates. We observe the noticeable deviation of the stochastic results denoted by symbols from both the analytical prediction (4.24) (solid black line) and the results of the numerical solution of the system of ODEs (4.20)(solid blue line). In the regime of low travel rates, we not only expected, but also really observed similar behavior for the FKPP model. The analytical result (4.25) for the FKPP model (dashed line) does not reproduce numerics as well. Note that in the case of small local concentrations, we have a linear dependence on the travel rate, or equivalently, on the diffusion coefficient (Mai et al., 2000).

The deviation of the numerical solution from the Monte Carlo simulations occurs for small $\omega$, which are due to the finite number of agents we are considering. In the figure, the crossover from the linear scaling with $\omega$ to the numerically obtained one is also noticeable. It can be 


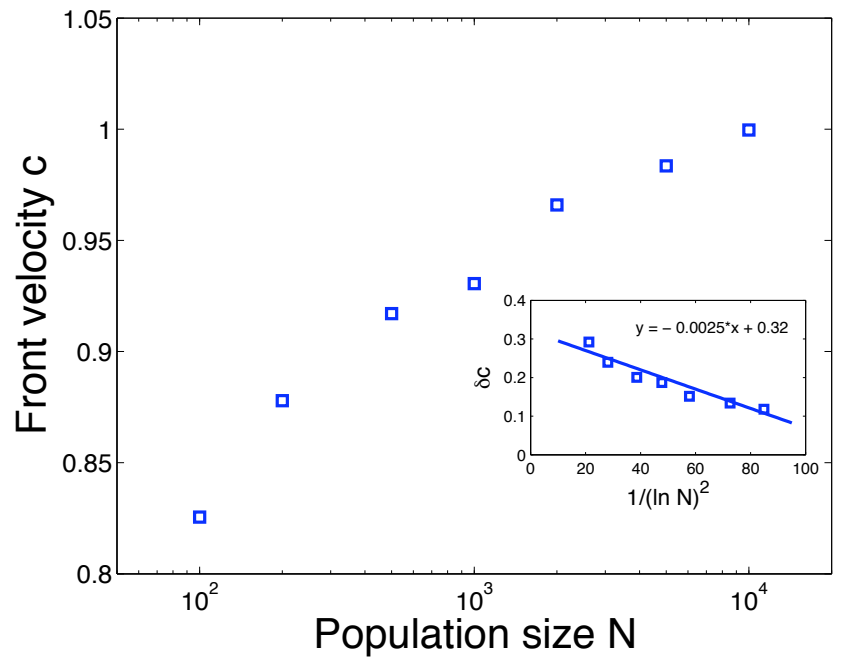

Figure 4.13: Dependence of the front velocity $c(N)$ on the number of individuals pro site $N$ (the SI model with $\alpha=1$ and $\omega=10$ ). In the inset, the deviation $\delta c$ of the velocity from the mean-field prediction in dependence on $1 /(\ln N)^{2}$, is presented. One can observe a good agreement with prediction $\delta c \sim 1 /(\ln N)^{2}$ (Brunet and Derrida, 1997).

interpreted as follows: if we consider very small travel rates - thus the infection rate is very large $(\alpha \gg \omega)$ - an outbreak takes place almost instantaneously and the epidemic essentially hops from an affected location to non-affected one. It also explains why the results for small travel rates for the recurrent system, and for the reaction-diffusion, are the same. The rate of such hopping is proportional to $N \omega$, i.e. to the flow of individuals between locations, where $N$ is a number (or average number in the FKPP case) of individuals pro site. This is illustrated in Figure 4.15. The crossover from discrete to continuous behavior takes place when $\alpha \sim \omega N$, i.e. $\omega_{\text {crossover }} \sim \alpha N^{-1}$.

Note that the slow convergence of the velocity towards zero with decreasing travel rate can be understood from the following considerations. Let us consider just two locations with agents which can hop between them. Without loss of generality we consider the SI epidemic. At the beginning of the epidemic the number of infectives in the second location is small and we can linearize the standard SI kinetic equation (2.17)

$$
\frac{d j_{2}}{d t}=\alpha j_{2}+\omega j_{1}
$$

where we have neglected the backward flux of the individuals from the second location. By $j_{1}$ and $j_{2}$, we have denoted concentrations of infectives in first and second locations. This equation can be integrated by means of the integrating factor

$$
j_{2}(t)=e^{\alpha t} \int_{0}^{t} d \tau j_{1}(\tau)
$$

The solution of the SI model for the first location (as if it was isolated) reads

$$
j_{1}(t)=\frac{1}{1+a e^{-\alpha t}},
$$

where $a=\frac{1-j_{1}(0)}{j_{1}(0)}$. Inserting (4.51) into (4.50) and performing integration, we obtain

$$
j_{2}(t)=e^{\alpha t} \omega\left(\ln \frac{1+a e^{-\alpha t}}{1+a}+\ln e^{\alpha t}\right)
$$




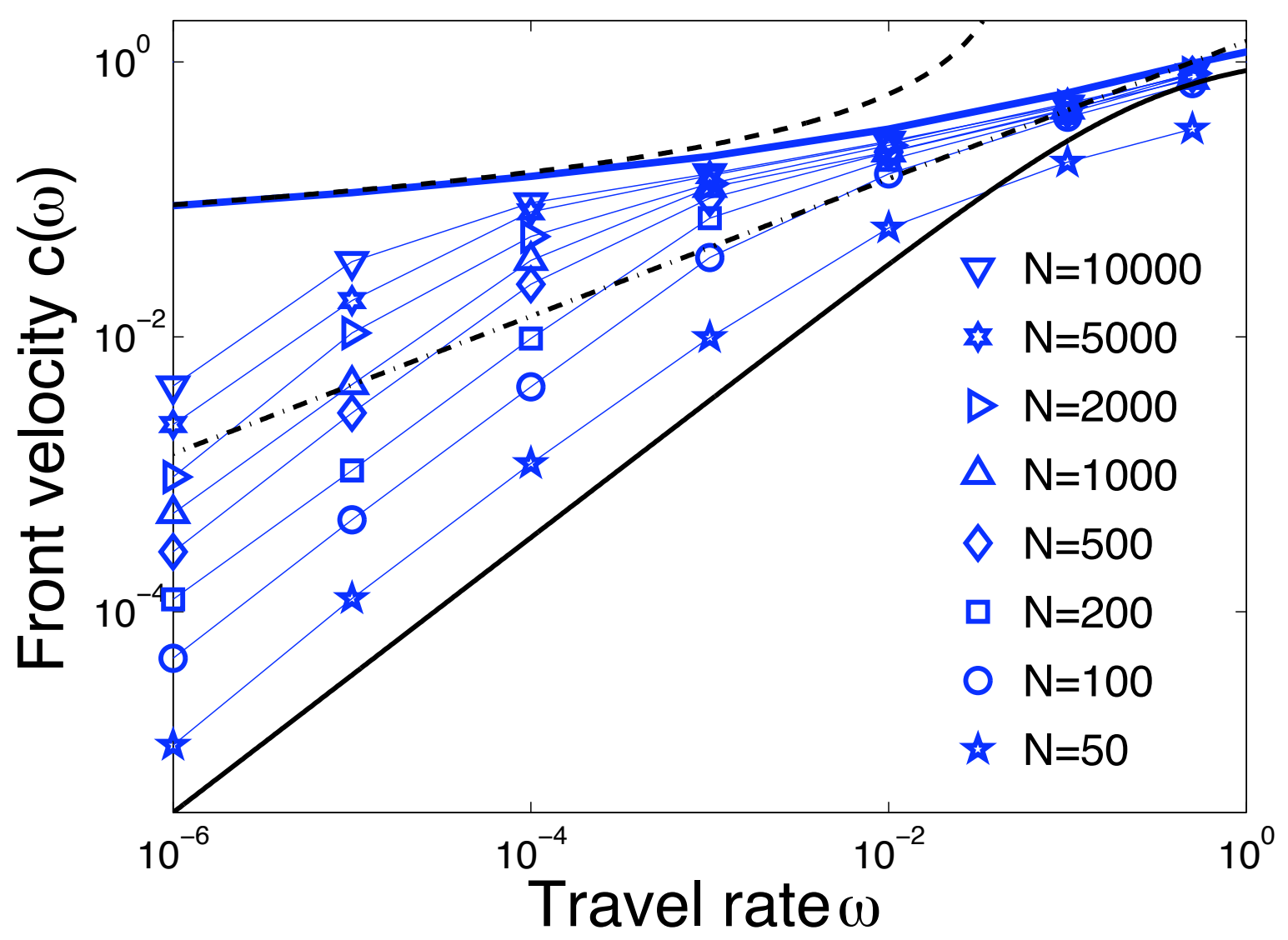

Figure 4.14: Front velocity dependence $c(\omega)$ on low travel rates. One observes deviations of the front velocity for low travel rates from the analytical predictions (4.24). The thick blue line denotes the velocity obtained by the numerical solution of the mean-field equations (4.20). Symbols denote results of the numerical simulations for different number of individuals per location $N$. The dashed curve is the analytical result (4.52). The dependence $c(\omega)$ in the FKPP case is denoted by the dashed-dotted line.

For large times $\ln \left(1+a e^{-\alpha t}\right) \approx 0$, the exponential growth overcomes the logarithmic one and thus

$$
j_{2}(t) \sim \omega e^{\alpha t} .
$$

If we are interested in the lag time between outbreaks of the epidemics, i.e. $\Delta \tau(q)=\tau_{2}(q)-$ $\tau_{1}(q)$, where $\tau_{1}$ and $\tau_{2}$ are times moments at which the concentrations of infectives attains some particular value $q$ in the first and second location, then we have

$$
\Delta \tau(q) \sim \ln \frac{q}{\alpha \omega}-\ln \frac{q}{1-q}-\ln a,
$$

where we have used an expression for the $t\left(j_{1}\right)$ dependence for a SI model which can be easily derived from (4.51). Choosing $q=1 / 2$, we obtain for the velocity $c=\Delta \tau^{-1}$

$$
c \sim\left(-\ln \frac{\alpha \omega}{2}-\ln a\right)^{-1}
$$




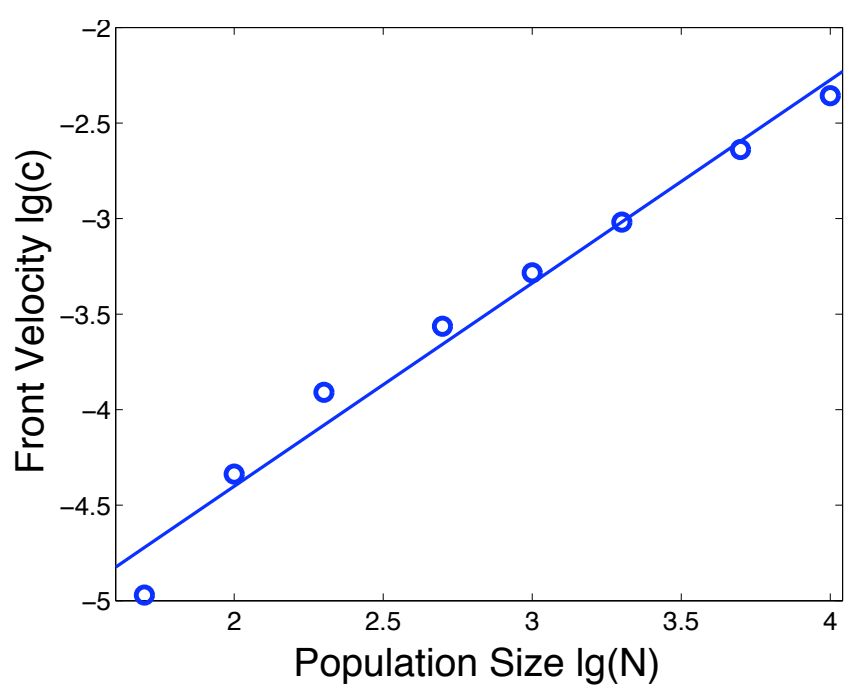

Figure 4.15: Dependence $c(N)$ of the front velocity on the number of agents pro site for small travel rates $(\omega=$ $10^{-6}$ ) as obtained by stochastic numerical simulations. The solid line is the fit $\lg c=1.06 \lg N-6.51$ or $c \sim N^{1.06}$.

This expression gives the slow convergence towards zero, which is in agreement with the results obtained by numerical solution of the system of ODEs. From (4.52), for $\omega \rightarrow 0$, the velocity falls to zero as expected.

One can conclude that, the numerical solution of the ordinary differential equations (4.20) on a lattice describing the epidemic due to the recurrent transport as well as the simulation of the full stochastic model defined by the system of reactions (4.19) support the theoretical results obtained by analysis of the continuum equations (4.22) very well. A finite number of agents result in deviation of the front velocity towards smaller values. The deviation scales according to the Brunet-Derrida model in the case of moderate and high travel rates. In the case of low travel rates, the velocity depends linearly on the number of agents pro site. In low travel rates regime with increasing number of agents, we obtain a crossover from the linear dependence on the travel rate to a very slow inverse logarithmic convergence of the velocity towards zero with decreasing travel rate. We explained this dependence by considering the inverse of the delay time between epidemic outbreaks of just a pair of locations.

\subsection{Epidemics on networks}

In the previous sections, we have considered epidemic spread due to recurrent host movements on a chain and obtained corresponding continuum equations. However, the one-dimensional support constitutes the simplest possible setting. All real situations exhibit much more complex, network-like topologies for interconnectivity structure of different locations. In (Gastner and Newman, 2006), some real transportation networks were considered, e.g. highway network in the USA is reminiscent of a random Erdôs-Rényi (ER) network, while air transportation network is reminiscent of a small-world, scale-free network (Watts and Strogatz, 1998; Albert and Barabási, 2002).

In the context of epidemiology, there were numerous attempts to incorporate transportation networks, e.g. such as aviation or road networks. However, either dispersive, e.g. (Hufnagel et al., 2004) or direct coupling approaches, e.g. (Camitz and Liljeros, 2006) were consid- 
ered. Note that the success of the random walk approach in the SARS case (Hufnagel et al., 2004) can be explained by the small world feature of the global aviation network and small incubation period of the disease. In Section 4.3, we have seen that the eigenvalues of both epidemics due to random walk and recurrent travel coupling coincide if individuals are equally distributed over locations. In Section 4.5.2 we have found that very slow travel rates lead to the same front velocity in both recurrent and random walk models. The success of the direct coupling model is due to the frequent commuting over short distances, because, as we have seen in Section 4.2 the model with recurrent movements is equivalent to the direct coupled one in this case.

Thus, a question arises if, and how, our results from previous sections can be extended to the case of complex network topologies. In the present section, we discuss a general case of networks, with nodes representing locations, and edges representing connections between them. Generally expect that the effect of attenuation of the speed of the epidemic wave as compared with the random walk travel pattern becomes less pronounced if we consider network topology. In general, much more links and thus "escape" routes for epidemic are available and recurrent movements play less important roles. This is confirmed in Figure 4.16, where for both random walk and recurrent travel, the dependence of the fraction of infectives in the SI epidemic is presented for one dimensional chains with coupling to different number of the next neighbors $k=1,2$ and 10. However, the detailed exploration of the parameter space and extensive stochastic simulations may reveal new unexpected effects. One of the important questions which need to be answered is about the dependence of the effective basic reproduction ratio on particular network properties (Arino and van den Driessche, 2003).

Another interesting aspect we will dwell on, is disorder, which can be very important for various spreading phenomena. In Chapter 3, we have already seen that disorder can lead to acceleration of the superdiffusive processes (Belik and Brockmann, 2007). The recent study of the SARS-epidemic on the aviation network (Hufnagel et al., 2004) indicates that the disorder in travel rates can increase the predictability of the epidemic dynamics. Which role disorder or inhomogeneity plays in the dynamics of the spreading phenomena in the metapopulation network with bidirectional movements of individuals, remains an open question. As opposed to the regular lattices, random metapopulations networks permit two kinds of disorder. The first one is immanent in the random topology due to different degrees of nodes. The second one is inherent in different population sizes or capacities of single nodes. Certainly, the two types of disorder cannot be separated. They are actually always interwoven. We will propose several ways to incorporate disorder into our model and relate it to the empirical data.

\subsubsection{Modelling travel rates}

In Section 3.1, in which we have defined the model for bidirectional travel on star-like topologies, we have introduced forward and backward rates $\omega_{m n}^{n}$ and $\omega_{n m}^{n}$ for species belonging to the node $n$. For a homogeneous lattice with travel only to the next neighbor locations, it was a rather straightforward assumption to consider just two different values for the forward and backward rates, $\omega_{1}$ and $\omega_{2}$ (cf. Section 4.4). In the case of travel to further locations and inhomogeneous lattices, it is not obvious how to incorporate the dependence of the travel rates on e.g. distance from the home location or on population sizes of home and remote locations. 


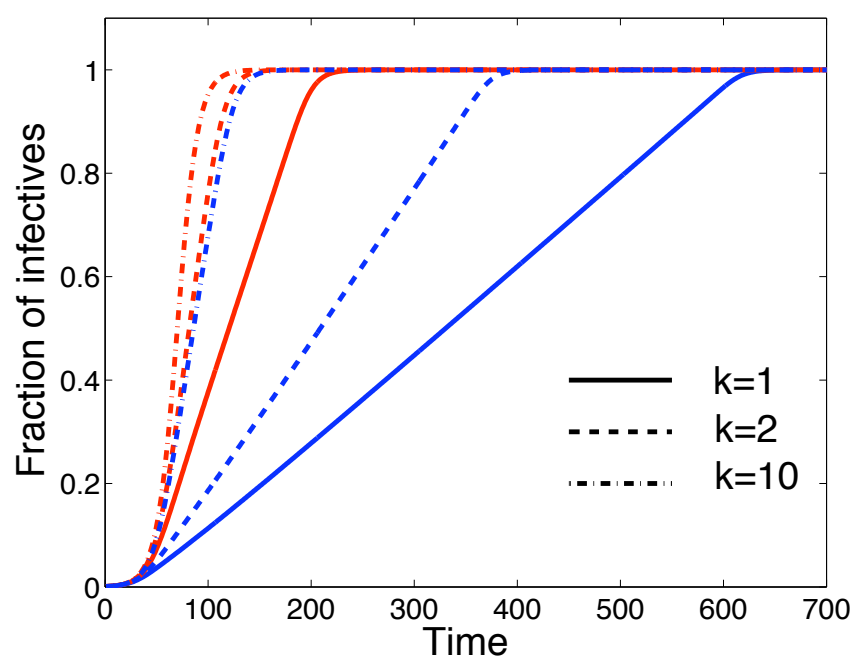

Figure 4.16: The dependence of the time-course of the SI epidemic on the number of nearest neighbours $k$, allowed for agent movements. The random walk travel model (red lines) and the bidirectionally coupled model (blue lines) are compared. Infection rate, $\alpha=1$ and travel rate, $\omega=1$.

Information usually available are just flows of individuals, e.g. humans, among different locations without specification to which location they belong to. Assessment of fluxes of humans is possible by indirect methods alongside with a direct census, as done e.g. by survey authorities ${ }^{10}$. For example, one can use some entities as movement trackers: money bills (Brockmann et al., 2006) or trackable items moving between geocaches (Brockmann and Theiss, 2008b). ${ }^{11}$ The data on flows were frequently used to get transition probabilities under the assumption of random walk travel (Hufnagel et al., 2004) or not precisely (cf. Section 4.2) to get coupling strengths (Viboud et al., 2006; Camitz and Liljeros, 2006) in epidemiological models. Even if the flows are available, one cannot uniquely determine the travel rates. It is not enough to know the flows of individuals among different locations to determine the parameters $\omega_{m n}^{n}$. Generally for every location $n$, we have $3 \times k_{n}$ unknown parameters, where $k_{n}$ is the number of neighbor locations. These include forward and backward return rates $\omega_{k n}^{n}, \omega_{n k}^{n}$ and stationary distribution of the individuals over different locations $N_{n}^{k}$. The flows between locations can be imaginably split into two flows of individuals belonging to different cities. Indeed, the flow between two locations $m$ and $n$ consists of two terms:

$$
F_{n m}=\omega_{n m}^{n} N_{m}^{n}+\omega_{n m}^{m} N_{m}^{m} .
$$

The first term describes individuals which belonging to location $n$, have visited location $m$ and are returning to their home location $n$. The second one describes individuals who, belonging to the location $m$, leave it for location $n$. Swapping the indices $m$ and $n$ gives the flow in the opposite direction $F_{m n}$. Assumption of the detailed balance leads to $F_{n m}=F_{m n}$. Additionally, the detailed balance has to be fulfilled separately for individuals from a particular location:

$$
\omega_{n m}^{n} N_{m}^{n}=\omega_{m n}^{n} N_{n}^{n}
$$

\footnotetext{
$10_{\text {WwW }}$.bts.gov, www . destatis.de

${ }^{11}$ Geocaches are usually some collections of small objects (travel bugs) which are hidden at some picturesque places and supposed to be found with a GPS navigation divice. Their coordinates are published on the internet. Geocashing is a modern version of fox hunting with radio devices. It offers a lot of fun for its devotees. See also www. geocaching. com
} 
Moreover, the conservation of the population sizes implies

$$
\sum_{m \neq n} N_{n}^{m}=N_{n}
$$

Hence, we have $2 \times k_{n}+1$ conditions on $3 \times k_{n}$ parameters and thus cannot determine them uniquely. As more data of high resolution on human or animal movements become available from e.g. mobile phone tracking (González et al., 2008) or animal telemetry ${ }^{12}$ (Cooke et al., 2004), the direct assessment of travel rates may even become possible. But until then, we are predestined to make some assumptions on the values of the travel rates or use only available information. We can propose a zoo of different models incorporating ingredients such as geographical distance and local population sizes.

\subsubsection{Topological disorder}

First, let us consider the situation when the travel rate of all individuals is equal to $\omega$ and initial population sizes on every node are the same $N_{n}(t=0)=N_{0}$. Commuters are equally distributed among all neighbor locations. As we have already mentioned in Section 3.1, if we observe our population system with only travelling dynamics for a sufficiently long time, the steady state will be attained with the following real population sizes (4.6)

$$
N^{n \star}=N_{0}\left[\frac{1}{1+k_{n}}+\sum_{m=1}^{k_{n}} \frac{1}{1+k_{m}}\right]
$$

as compared to $N^{n \star} \sim N_{0}$ for the random walk travel with an equal travel rate (David and Brockmann, 2009) and $N_{n}^{\star} \sim N_{0} k_{n}$ in the case $\omega_{n m} \sim k_{m}^{-1}$ (Collizza et al., 2007). The (in)dependence of $\omega$ on the nodes degree is not a trivial question even in the case of ordinary random walk travel. Thus, given the degree distribution $p(k)$ and possible correlations among neighbors, the distribution of real population sizes $p(N)$ can be assessed in principle. Note that (4.54) witnesses greater variability in the population sizes than initially given ones. This may lead to greater variability in the overall time course of the epidemics. In Figure 4.17, the equilibrium cumulative distribution of the population sizes $F\left(N / N_{0}\right)$ for random Erdôs-Rényi networks with the average degree $\langle k\rangle=10$ and with 100 nodes is presented. From (4.54), one can approximate

$$
N / N_{0} \approx k /\langle k\rangle \text {. }
$$

Hence, for a Erdős-Rényi network with the nodes degree distribution given by the Poisson law $p(k)=e^{-\langle k\rangle}\langle k\rangle^{k} / k$ ! (Albert and Barabási, 2002), we can calculate the cumulative distribution of the stationary population sizes

$$
F\left(\frac{N}{N_{0}}\right) \approx \frac{\langle k\rangle}{e} \sum_{n}^{k \leq N / N_{0}} \frac{1}{n !} .
$$

From (4.55), is clear that the variance of the equilibrium population sizes decreases with increasing average node degree $\langle k\rangle$. Thus, large connectivity leads to smoothing of the real population sizes. Real populations become more homogeneous.

\footnotetext{
$12_{\text {WWW }}$ movebank. org
} 


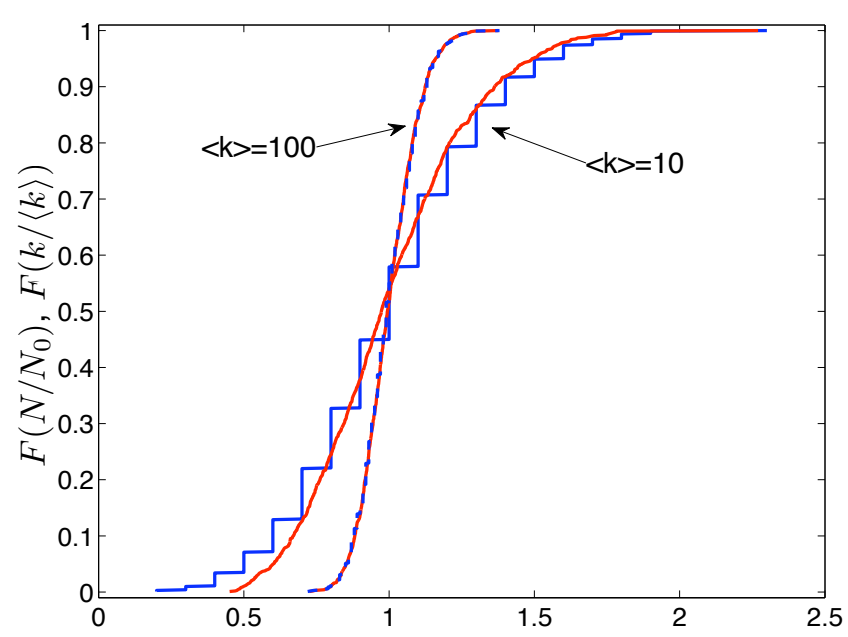

Figure 4.17: The dependence of the equilibrium population size distribution $F\left(N / N_{0}\right)$ (red line) on the average node degree $\langle k\rangle$ of the random Erdős-Rényi network with 1000 nodes. Blue lines represents the distribution $F(k /\langle k\rangle)$. According to (4.55) $F\left(N / N_{0}\right) \approx F(k /\langle k\rangle)$.

Having considered the consequences of network topology in a homogeneously populated network, we will propose models for populational or capacity disorder. One important assumption concerns the backward travel rate $\omega_{n m}^{n}(n \neq m)$ which can be set to a constant $\omega_{n m}^{n}=\omega^{-}$. This implies that all individuals spend almost the same amount of time at their non-home places. This assumption was also used in (Keeling and Rohani, 2007).

\subsubsection{Equal commuter ratios independent of the location}

One can argue that if individuals, in particular humans, decide to move to some location, they take the population size (alternatively, one could take the capacity) of the destination into account. If there are many of individuals in a remote place, there are also more appealing conditions. Also, in order to choose one location from many alternatives, an individual needs to consider the relative size of the destination. If we assume that the forward rate is proportional to the relative size of the distal node, we can write down

$$
\omega_{n k}^{k}=\omega^{+} \frac{N_{n}^{n} A_{n k}}{\sum_{m} N_{m}^{m} A_{m k}}
$$


with the adjacency matrix $\mathcal{A}^{13}$. This leads to condition $\sum_{n \neq k} \omega_{n k}^{k}=\omega^{+}$and thus we guarantee equal total commuter ratio

$$
\varepsilon_{k}=\sum_{n \neq k} N_{k}^{n} / N_{k}^{k}=\omega^{+} / \omega^{-}
$$

for all locations. Because $N_{k}^{k}=N_{k}\left(1+\varepsilon_{k}\right)$ and $\varepsilon_{k}=\varepsilon_{0}$ is equal for all nodes, we obtain $\omega_{n k}^{k}=\omega^{+} \frac{N_{n} A_{n k}}{\sum_{m} N_{m} A_{m k}}$. Thus, there is no difference which population size, actual $N^{k}$ or nominal $N_{k}$, we consider in (4.56). In the spirit of the previous sections where epidemics due to random walk and to recurrent travel were compared, we can calibrate these two models so that the total flows between locations remain the same. To calculate the total flow, we first need to know the partial flow of the $k$-species from $k$ to $n: F_{n k}^{k}=\omega^{+} \frac{A_{m k}}{\sum_{m \neq k} A_{m k}} \frac{N_{n} N_{k}}{1+\varepsilon_{0}}$. Thus, the total flow from $k$ to $n$ is given by

$$
F_{n k}=\omega^{+} \frac{N_{n} N_{k}}{1+\varepsilon_{0}}\left(K_{k}^{-1}+K_{n}^{-1}\right) .
$$

Note the condition $N_{n}^{k}=N_{k}^{n}$ is not fulfilled in this case. The last expression resembles the common condition for diffusion on graphs with $w \propto K^{-1}$. In the case of a regular lattice, $F_{n k}$ reduces to

$$
F_{n k}=\omega^{+} \frac{N_{n} N_{k}}{1+\varepsilon^{0}} 2 K_{0}^{-1}
$$

where $K_{0}$ is a number of neighbors of one lattice site.

However, the normalization over the size of the neighborhood may not be always important. Sometimes there is no information available and e.g. only the distance plays a role.

\subsubsection{Non-equal commuter ratios without normalization}

Another model for the forward travel rate can be proposed, where no averaging over neighborhood is performed

$$
\omega_{n k}^{k}=\gamma \omega^{+} N_{n} A_{n k} .
$$

For the travellers ratio, we have

$$
\varepsilon_{k}=\gamma \frac{\omega^{+}}{\omega-}\langle N\rangle_{k}
$$

\footnotetext{
${ }^{13}$ Random networks or graphs are defined as a set of nodes with edges or links between them. Information whether two particular nodes are connected is given by the adjacency matrix $\mathcal{A}$

$$
a_{i j}= \begin{cases}1 & \text { if } i \text { and } j \text { are connected } \\ 0 & \text { if } i \text { and } j \text { are not connected or } i=j\end{cases}
$$

It is obvious that if we postulate symmetric links, i.e. if $i$ is connected to $j$ then $j$ is connected to $i$ as well, the adjacency matrix $\mathcal{A}$ is symmetric. One can consider different types of networks corresponding to different adjacency matrices. To generate a particular network means to generate a particular kind of the adjacency matrix.
} 
where $\langle N\rangle_{k}=\sum_{n} N_{n} A_{n k}$ is the size of the neighborhood of $k$ and thus $\varepsilon_{k}$ is generally different for different $k$. Then the partial flow reads $F_{n k}^{k}=\omega_{n k}^{k} N_{k}^{k}=\gamma \frac{\omega^{+} N_{n} N_{k}}{1+\gamma \frac{w^{+}}{w^{-}}\langle N\rangle_{k}}$ and hence

$$
F_{n k}=\gamma \omega^{+} N^{n} N^{k}\left(\frac{1}{1+\gamma \frac{\omega^{+}}{\omega-}\langle N\rangle_{n}}+\frac{1}{1+\gamma \frac{\omega^{+}}{\omega-}\langle N\rangle_{k}}\right) \text {. }
$$

For $\gamma$, one can assume e.g. the global averaging $\gamma \propto \frac{1}{\langle N\rangle}$, where $\langle N\rangle=\frac{1}{M} \sum_{n} N_{n}$ or introduce the distance dependence $\gamma_{k n} \propto r_{k n}^{-d-\mu}$, where $r$ is the distance between $k$ and $n, d$ is the space dimension and $\mu$ is the exponent responsible for either short or long-range movements. This model can be applied to spatially embedded networks.

\subsubsection{Rates dependent on the actual population sizes}

In the previous models, we have used travel rates proportional to the nominal population sizes. One can also try to use the actual size of population that remains at home

$$
\omega_{n k}^{k}=\gamma \times \omega^{+} N_{n}^{n} A_{n k}
$$

where e.g. $\gamma=\frac{1}{K_{k}\left\langle N_{h}\right\rangle}$ and $K_{k}=\sum_{n \neq k} A_{n k}$ is the degree of the node $k$ and $\left\langle N_{h}\right\rangle=$ $\frac{1}{M} \sum_{n=1}^{M} N_{n}^{n}$ is the average number of people staying at home in the entire population. With these assumptions we obtain for the $\varepsilon_{k}$ that determine the equilibrium

$$
\varepsilon_{k}=\frac{\omega^{+}}{\omega^{-}} \times \frac{\left\langle N_{h}\right\rangle_{k}}{\left\langle N_{h}\right\rangle}
$$

where $\left\langle N_{h}\right\rangle_{k}=\frac{1}{K_{k}} \sum_{n \neq k} N_{n}^{n} A_{n k}$ is the average number of people staying at home in the locations accessible from $k$. Note that in the expression for $\varepsilon_{k}$ the second fraction is of the order of one, whereas the first fraction in small. Note however, that, if we assume (4.57), we need to know

$$
N_{n}^{n}=N^{n} /\left(1+\sum_{m} \omega_{m n}^{m} / \omega^{-}\right)
$$

which is in turn is determined by some other $w_{n^{\prime} n}^{n^{\prime}}$. In principle, one can try to resolve these dependencies self-consistently, starting with some initial distribution of the real home populations $N_{n}^{n}$, obtaining $\omega_{k n}^{k}$ from (4.57), then gain $N_{n}^{n}$ from (4.58) and so forth. Thus real home population sizes lead to inconsistencies which need to be resolved.

\subsubsection{Available flows}

If the flows between locations are already known, one possibility to assess the travel rates is to fix the fraction of individuals who are away at the moment, over individuals who stay at home, i.e. to fix the already introduced commuter ratio (cf. Section 4.4):

$$
\varepsilon_{k}^{m}=\frac{N_{k}^{m}}{N_{k}^{k}} .
$$


E.g. one can set $\varepsilon_{n}^{m}=\varepsilon_{0} / k_{n}$, where $k_{n}$ is the number of neighbouring locations of the location $n$ (the nodes degree). Next, given the partial fluxes $\omega_{n m}^{n} N_{n}^{m}=\omega_{m n}^{n} N_{n}^{n}=\gamma_{n m} F_{n m}$, we obtain for the backward rate which is now not fixed

$$
\omega_{n m}^{n}=\gamma_{n m} \frac{F_{n m} k_{n}}{\varepsilon_{0} N_{n}^{n}}
$$

and for the forward rate

$$
\omega_{m n}^{n}=\gamma_{n m} \frac{F_{n m}}{N_{n}^{n}}
$$

where from (4.53) and (4.59), $N_{n}^{n}=N_{n}\left(1+\sum_{m \neq n} \varepsilon_{0} / k_{n}\right)^{-1}$. For travel rates of individuals belonging to the city $m$, we should swap the indices and change $\gamma_{n m}$ to $1-\gamma_{n m}$. The simplest possibility is to assume equal partial flows $\gamma=1 / 2$. Further assumptions can be made on how $\varepsilon_{n}^{m}$ and $\gamma_{n m}$ are related to degree and size of the nodes.

The next step should be the comparison of the models with random walk and recurrent movements using the proposed models for the travel rates. The interlocation flows should be kept the same in both models. In the random walk case, the transition rates are given through $\omega_{k n}=F_{k n} / N_{n}$. One of the important questions is whether the network topology influences the epidemics due to recurrent travel pattern in another way than epidemics due to random walk travel pattern under disordered conditions. Two simple extreme cases are obvious. In the all-to-all coupling network, there is almost no difference in the dynamics for the commuter ratio corresponding to the equal time spent in neighboring and home locations. In the case of regular lattice, the difference is most pronounced. One can argue that all other topologies, including random networks, leads to intermediate behavior between these two extreme cases.

\subsection{Perspectives}

So far, we considered the recurrent transport mechanism in metapopulational epidemic systems, related this to the directly coupled model and analysed the continuum approximation. However, there is still more to investigate on. Generalizations to two-dimensional support and incorporation of long-range movements is still an open question. We argue, that in the latter case the attenuation (as compared with the random walk mechanism) of the epidemic spread is reduced. In the continuum limit, one can take into account fluctuations using field theoretical methods (Doi, 1976b,a; Kree et al., 1989) or moment closure methods (Parham and Ferguson, 2006). Thus far, we considered recurrent movements which are Markov processes. One can also introduce memory for individuals, prohibiting visits to locations visited in the immediate past. Even periodic movements corresponding to clear home-work rhythm could be considered. The dynamics on network topologies need to be investigated in detail using the models for travel rates incorporating inhomogeneity and spatial properties as proposed in Section 4.6.1. It is important to know how network properties such as community structure and modularity (Newman, 2006; Watts et al., 2005) influence epidemiological dynamics with recurrent transport. Within our framework, we can easily allow travel not only to next-neighbor locations and returns over remote nodes. The problem of the limited length percolation (López 
et al., 2007) is also relevant in this context. Inhomogeneity of the human population (Liljeros et al., 2001; Pastor-Satorras and Vespignani, 2000; May and Lloyd, 2001; Sokolov and Belik, 2003) could also be addressed. More complex kinds of epidemiological dynamics (Grenfell et al., 2001) leading to synchronisation in the course of epidemic among different locations require also inter-patch correlations to be considered (Hagernaas et al., 2004). Note, that recurrent movements are not only human specific behavior but could be pertinent to animal systems as well.

The recurrent movements play a role not only in the epidemiological systems. There are also many other situations where this mechanism may be encountered. One of possible applications of the recurrent movements is the transport of some items analogous to the transport of pathogens in epidemiology. It can be e.g. money bills (Brockmann et al., 2006) or - as previously mentioned — trackable items from geocaches (Brockmann and Theiss, 2008b). A large field of of biological research is devoted to seed dispersal (Nathan, 2006). Seeds cannot move by themselves and need to be transported by wind or herbivorous animals. Let us look at the model for items dispersal due to recurrent movements more closely and compare it to the random walk model.

We consider a situation in which items can be left somewhere independently of the individuals who transport them (seeds) and a setting in which items cannot be separated from the individuals (some parasites, money bills). We consider a linear chain of locations with the next-neighbour coupling. The first reaction system with an underlying random walk pattern of individual movements reads:

$$
\begin{aligned}
A_{n}+G_{n} & \stackrel{\alpha}{\longrightarrow} A g_{n} \\
A g_{n} & \stackrel{\beta}{\longrightarrow} A_{n}+G_{n} \\
A_{n} & \stackrel{\omega}{\rightleftharpoons} A_{n \pm 1} \\
A g_{n} & \stackrel{\omega}{\rightleftharpoons} A g_{n \pm 1},
\end{aligned}
$$

where we denote the individuals without items by $A_{n}$, the individuals with items by $A g_{n}$ and the items themselves by $G_{n}$. The second model accounts for recurrent movements of individuals:

$$
\begin{array}{rll}
A_{n}^{n}+G_{n} & \stackrel{\alpha}{\rightarrow} A g_{n}^{n} \\
A_{n \pm 1}^{n}+G_{n} & \stackrel{\alpha}{\rightarrow} A g_{n \pm 1}^{n} \\
A g_{n}^{n} & \stackrel{\beta}{\rightarrow} A_{n}^{n}+G_{n} \\
A g_{n \pm 1}^{n} & \stackrel{\beta}{\longrightarrow} A_{n \pm 1}^{n}+G_{n} \\
A_{n}^{n} & \stackrel{\omega}{\rightleftharpoons} A_{n \pm 1}^{n} \\
A g_{n}^{n} & \stackrel{\omega}{\rightleftharpoons} A g_{n \pm 1}^{n},
\end{array}
$$

where $A_{m}^{n}$ and $A g_{m}^{n}$ are the numbers of individuals with and without items, belonging to the location $m$ and being now in the location $n$. In Figure 4.18, the concentrations of items initially 
situated in the first location at different times are presented. Red lines corresponds to the first random walk model, the blue one - to the second recurrent model. One can see, that as expected, random walk movements pattern leads to stronger dispersal due to the same reasons as in the case of epidemics. The interchange of items or pathogens depends on the probability of being at a particular location and it does not change significantly with increasing travel rate in the recurrent process. To quantitatively assess the model with items dispersal, one can try to use methods which were already successfully applied for various reaction-diffusion processes.

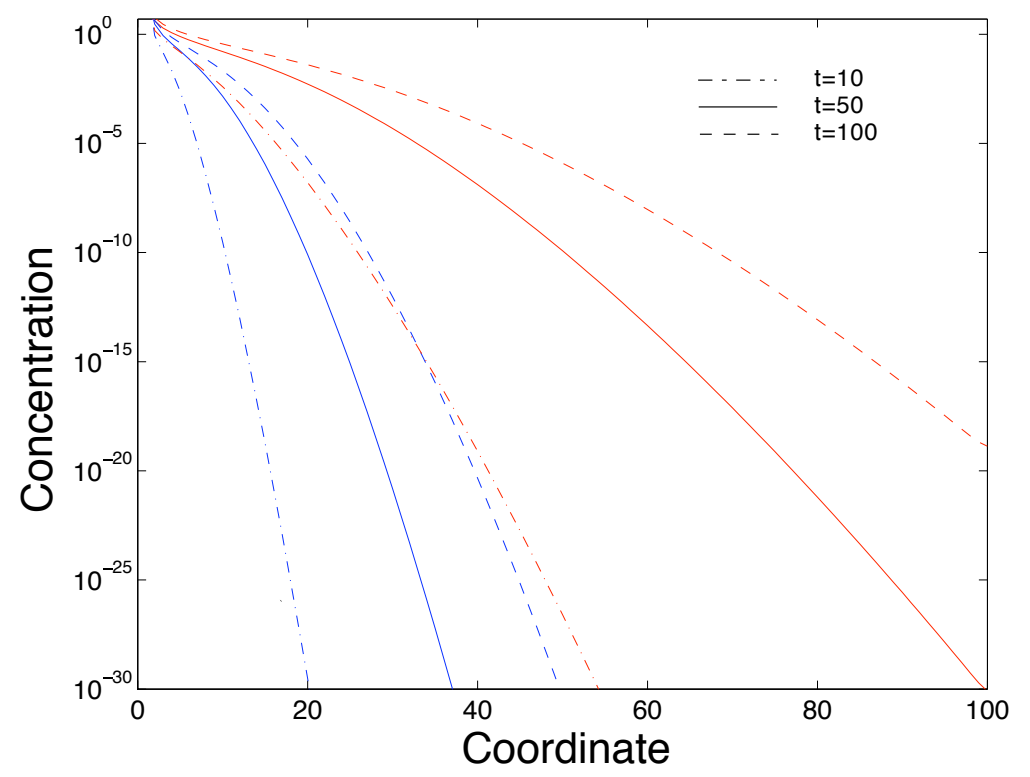

Figure 4.18: Concentration of different items transported by agents performing random walk (red lines) or recurrent movements (blue lines) at different times due to numerical solution of the mean-filed kinetic equations corresponding to (4.60) and (4.61) on a chain with 100 sites. Parameters are $\alpha=\beta=\omega=1$ and initial conditions $G(x=0, t=0)=$ 1000 .

Furthermore, our model with origin-dependent transition rates may be relevant for various dispersal phenomena (Clobert et al., 2003), i.e. spread of species or invasion into new habitats, such as the spread of genes (Novembre et al., 2008), influenza viruses (Russell et al., 2008) or infectious vectors (Tatem et al., 2006). Miscellaneous ecological spatially extended systems with interacting species, such as prey-predator or Lotka-Volterra systems (Volterra, 1926; Murray, 1993) are also of interest. Our approach can be applied even to such an exotic phenomena as rumour spread (Boccaletti et al., 2006). In this context, various self-organisational, non-equilibrium critical phenomena occurred (Horsthemke and Lefever, 2006; Stollenwerk and Jansen, 2007), which can be described in the game-theoretical framework (Nowak, 2006; Reichenbach et al., 2007) or by field-theoretical methods (Kuzovkov and Kotomin, 1988). We expect to find similar phenomena also in our framework.

The field research, i.e. the empirical assessment the parameters of the models, is of utmost importance and needs to be extended in future. Alongside with the direct methods such as census surveys or tracking of individuals (González et al., 2008; Cooke et al., 2004) indirect methods based on tracking of auxiliary items, could provide a reasonable alternative (Brockmann et al., 2006; Brockmann and Theiss, 2008b).

We wanted to draw attention to presice consideration of agent movements - mediators of spread. Our approach was an attempt to capture essential features of human-mediated 
spreading phenomena. We hope that many other adequate models will be established in future.

\subsection{Summary and discussion}

In the present chapter, we derived from first principles the general model for the epidemics spread due to host movements among different locations with transfer rates dependent on the location of origin. Our model is able to take into account the recurrent and confined nature of human movements revealed by recent empirical studies (Brockmann et al., 2006; González et al., 2008). We have considered in detail the special case of recurrent host movements to next-neighbor locations on overlapping star-like topologies (Sattenspiel and Dietz, 1995). Analysis of the stationary properties of our model reveals that actual population size does not necessarily equal to nominal population size.

We compared our model to diffusion-reaction and direct coupling models. The dynamics of the total number of infectives in some location can be separated into the fully mixed reaction part and transport part. However, the transport part can be reduced to neither the reactiondiffusion nor to the direct coupling model without further assumptions (Section 4.2). The widely used direct coupling approach can be derived from our formalism in the case of high travel rates, when fast equilibration in respect to movements can be assumed. The coupling strengths can be immediately related to the travel rates and underlying topology but in nontrivial manner. However, travel rates are sometimes taken simply proportional to coupling strengths (Camitz and Liljeros, 2006). We established the relation between the direct coupling approach and the underlying travel pattern from first principles. We also found the range of validity of the direct coupling model. Note that similar considerations could be found in (Keeling and Rohani, 2007), although our approach is more systematic. Furthermore, we show that the diffusion mediated spread can also be described within our framework.

In Section 4.3, we investigated an exemplary situation of just two locations. Even in such a case, we observed the difference in the dynamics of epidemic outbreaks as compared to conventional model with indistinguishable diffusing hosts. If the backward and forward travel rates are equal, linear stability analysis reveals no difference between random walk and dynamics mediated by recurrent movements. This case also corresponds to equal time spent by hosts in both locations and implies the maximal synchrony between two populations, cf. (Keeling and Rohani, 2005). To assess the effect of recurrent movements, we have introduced the commuter ratio, defined as the ratio of the number of agents sojourning in the non-home location, to the number of agents staying at home. Varying the commuter ratio, we could obtain retardation of the epidemic in one location in respect to another location. If we start epidemic in the first location and the commuter ratio is less than unity, we obtain retardation in the second location because there are too few travellers to transfer the disease into the second location. We obtain the delay in the first location for the commuter ratio greater than unity: there are to many travellers and the disease is first transferred into the second location before it develops in the first one).

We derived the continuum approximation of our epidemic model on one-dimensional lattice in Section 4.4. Obtained equations are structurally different from the FKPP equations (Kolmogorov et al., 1937; Fisher, 1937), a continuum limit of the reaction-diffusion model. 
Reaction and diffusion cannot be separated in our case. In the case of high travel rates, from our equations, one could recover the equation derived as the continuum limit of the directly coupled model (see Section 4.2). Similar continuum limit equations in the direct coupling case were obtained phenomenologically in more general form in (Lopez et al., 1999), and recently in (Postnikov and Sokolov, 2007), although no connection of coupling strengths in continuum limit to microscopical travel mechanism have been considered so far. We filled this gap and showed that the latter model (Postnikov and Sokolov, 2007) is a special case of our approach in the limit of infinite commuter ratio and immobile susceptibles.

We have obtained the analytical expression for the velocity of the epidemic wave in Section 4.4.1. The analysis shows while in the ordinary case with random walk travel pattern (FKPP case), we have the front velocity growing unboundedly with the travel rate. In the case of recurrent host movements, however, the velocity saturates towards its asymptotic value. In other words, the velocity is bounded from above. This result is one of the main consequences of the recurrent transport mechanism for the epidemic spread.

We have also investigated the dependence of the velocity on the commuter ratio and infection rate in Sections 4.4.4 and 4.4.5. Our theory predicts saturation of the velocity with increasing parameter values. Deviations between analytical predictions and results of numerical solutions of corresponding ordinary differential equations appear for low travel rates, small commuter ratio and high infection rates. Analytics reproduces numerics perfectly in the range of small infection rates. However, contrary to the analytically predicted saturation of the velocity with the growing reaction rate, numerical solutions reveal the unbounded dependence of the velocity on the reaction rate. That seems to be an artifact of the continuous approximation, i.e. a continuous density of individuals implying infinitive number of agents. The flow of agents is proportional to the number of them times the travel rate, and thus in the system with an infinite number of agents there is no restriction on the velocity from movements of single agents. The only restriction is the reaction rate. This is not true any more for a discrete system with a finite number of agents where an upper bound on the velocity given by the agents flow has to exist. Note, that the unbounded front velocity dependence on the inflection rate comes out in the FKPP case as well.

Alongside with the velocity of the epidemic front, another frequently considered quantity in the context of reaction-diffusion is the shape of the wave front. The standard approach to assess the front shape in the case of recurrent movements does not work. Calculations predict a non-monotonous dependence of the slope decay exponent on the travel rate. This is not confirmed by the numerical solutions of the corresponding ordinary differential equations. The slope of the front of the SI epidemic does not depend on the infection rate (or slightly depends in the SIS-case). This is in contrast to the analogous result for the ordinary FKPP model. Thus, for the front shape of the directl coupling model, the reaction rate plays almost no role. The velocity of the front and its shape stay in direct connection to each other. That is why the slope of the FKPP wave front diminishes to zero with the increasing movement rate contrary to the slope of the model with recurrent movements, which has a lower boundary.

We have solved numerically the ordinary differential equations on a lattice describing the epidemic due to the recurrent movements. We also performed the simulation of the full stochastic model. These numerical results supported very well our theoretical predictions obtained by the analysis of the continuum equations. However, the finite number of agents 
results in smaller values of front velocity than predicted theoretically. This deviation scales according to the Brunet-Derrida formula (Brunet and Derrida, 1997) in the case of moderate and high travel rates. In the case of low travel rates, the velocity depends linearly on the number of agents pro site. In the range of low movement rates with increasing number of particles, we obtained the crossover from the linear dependence of the front velocity on the travel rate to the very slow inverse logarithmic convergence towards zero with decreasing travel rate. The same effect can be observed in the FKPP case. We have explained this dependence by considering the inverse of the delay time between epidemic outbreaks in the case of two locations (Section 4.5.2).

In Section 4.6, we considered epidemics due to recurrent transport on network topologies with different nodes representing different locations. We proposed different models for transition rates of travelling species incorporating populational and topological disorder. Equal movement rates in the initially equally populated random network lead to large variability in the actual population sizes. This can lead to greater variability in the evolution of the epidemics. On the other hand, large connectivity leads to homogenization of actual population sizes. One of the important questions which need to be asked is, whether the network topology influences the epidemics due to recurrent travel pattern in different way than epidemics due to random walk travel pattern. To compare both mentioned above models we suggest to keep interlocation flows the same in both models and to use the above mentioned models for movement rates. 


\section{Conclusion}

In this thesis, we have studied different issues of spatial epidemic dynamics. We were mainly interested in the following problems. The first problem deals with superdiffusion in inhomogeneous environments (Chapter 3). The second problem concerns epidemics mediated by the recurrent movements of hosts (Chapter 4).

In the first part of the thesis, motivated by the empirical evidence of the superdiffusive nature of spreading processes in animal and human systems (Brockmann et al., 2006; Sims et al., 2008), we have investigated the role of disorder for Lévy flights in inhomogeneous environments. We have accounted for inhomogeneity by transition rates asymmetrically dependent on the start (source) and the destination (target) of a single jump event (Belik and Brockmann, 2007). Such a law of movements is reminiscent of the gravity model, used for a long time in economics and social sciences to model flows of goods or individuals between different inhomogeneously populated locations (Zipf, 1946; Wilson, 1967). Our model could also be considered as a generalization of the concept of topological superdiffusion (Brockmann and Sokolov, 2002).

Our analysis unveiled a striking difference in the behavior of ordinary diffusion and Lévy flights on large scales in the presence of inhomogeneities (Section 3.4). In the case of ordinary diffusion we can achieve only attenuation of the process. Remarkably, in the case of superdiffusion, dependent on the relative source and target impact not only attenuation but also acceleration of the process could be possible. This stands in contrast to common belief that disorder can only attenuate diffusive processes. For superdiffusive processes with growing target impact the attenuation diminishes until it gives place to acceleration after the critical target impact. Maximal acceleration is achieved when transition rate depends exclusively on the properties of target location. Empirical calibration of the gravity model supports this finding (Xia et al., 2004). Our results state that if autonomous agents aim to achieve the fastest dispersion, they need to move with only the target-dependent transition rates. This result can be heuristically explained using the concept of salience or attractiveness field. Ordinary diffusive walkers are only aware of their immediate surrounding and for most of the time remain trapped in confined regions. In contrast, as soon as non-local Lévy flights are allowed, random walkers move effectively between the regions of high attractiveness. This leads to an enhanced exploration of the environment and acceleration of the process. This result is universal and independent of the particular kind of inhomogeneity since we have analytically and numerically demonstrated that for different periodic and random environments.

Dynamics on small and intermediate scales reveals that acceleration of the superdiffusive processes has its price and could not be achieved on all scales (Section 3.3). While acceleration appears on large scales, attenuation occurs on small and intermediate scales. Ordinary diffusion and superdiffusion processes exhibit different levels of sensitivity to different types of inhomogeneity. For both ordinary diffusion and superdiffusion, on large scales, the sensi- 
tivity diminishes initially with increasing target impact. However, above the critical value of the relative source and target impact, their ways split. While the sensitivity of superdiffusion continues to increase with target impact, the sensitivity of ordinary diffusion decreases. An environment with seldom attractive regions disturbs superdiffusive processes to the largest extent. In contrast to the statement that ordinary diffusion is not affected by the particular shape of inhomogeneity (Brockmann, 2003), ordinary diffusion can distinguish between inhomogeneities with opposite bias in the case of disbalanced source-target impact.

In infinite systems, the response of the superdiffusive processes on large scales exhibits discontinuity at the Lévy index corresponding to ordinary diffusion. However, in finite size systems there is a continuous transition from superdiffusion to ordinary diffusion (Section 3.6). We demonstrate that convergence towards the infinite-size result depends on the superdiffusive exponent and on the source-target impact. Fast convergence implies less influence of inhomogeneity. Thus we show that, dependent on the source-target impact, different Lévy exponents, ranging from ordinary diffusive to superdiffusive, are optimal. We recover the alleged optimality of the intermediate Lévy exponents (Viswanathan et al., 1999b) as a special case of the balanced source and target influence.

In Section 3.7, we learn that the acceleration of superdiffusion vanishes as soon as we introduce normalization of the salience, and regions of high attractiveness become relative to the average level of attraction. Ordinary diffusion does not change its behavior qualitatively — its attenuation still monotonously increases with the source-target impact. In contrast the influence of source and target becomes non-monotonous and symmetrical for superdiffusion. Superdiffusive processes can no longer be accelerated and the most pronounced attenuation is attained for the balanced source-target impact. This results imply that if global information becomes available, the largest gain we can achieve due to variation of the source-target impact is zero attenuation. It is achieved for either exclusively source or target dependent processes. In this case the process spreads as in homogeneous media.

To apply our results to epidemiological problems we want to extend our investigation to the case of reaction-superdiffusion processes in the presence of quenched disorder. In Section 3.8, we outlined the way to do this using field theoretical techniques. Furthermore, the dependence of the optimal Lévy exponent on the source and target impact should be addressed in the context of random search strategies (Condamin et al., 2008). Other fields of application include various ecological systems, for example prey-predator interactions.

In the second part of the thesis we have looked for adequate models of human mediated dispersion. In particular we studied the impact of recurrent host movements on the spatial spread of infectious diseases. We were motivated by recent empirical studies (González et al., 2008; Brockmann et al., 2006) which provide evidence for the recurrent and confined character of human movements.

In Section 4.1, we built a general model for epidemic spread mediated by agents moving with origin-dependent transition rates. In contrast to indistinguishable agents of the reactiondiffusion model (FKPP), we distinguish our individuals according to their home place. The global steady state in such a system, is generally different from the steady state of the reactiondiffusion model. It is easy to generalize our model, allowing agents to change their home place to recover the reaction-diffusion approach. We considered the special case of recurrent movements to the next-neighbor locations on overlapping star like topologies (Sattenspiel 


\section{Conclusion}

and Dietz, 1995). Our analysis showed that the stationary actual population sizes do not necessarily coincide with the nominal sizes. We showed that the heuristic direct coupling approach, widely used to describe spatio-temporal epidemic dynamics constitutes a particular case of our model in the limit of high movement rates. In this case the fast equilibration of species in respect to transport could be assumed, and coupling strengths of the direct coupling model could be explicitly related to the movement rates and the underlying topology. Our results demystify the heuristically introduced direct coupling model and show its range of validity. Coupling strengths are often assumed to be just proportional to travel rates (Camitz and Liljeros, 2006), although our study shows that generally this is not true.

The minimal number of locations for the effect of recurrent movements already appears is two (Section 4.3). Let us note that we have used much general and systematic approach than in the study on the similar subject (Keeling and Rohani, 2005) performed independently from us. In this simplest setting the strongest synchronization between populations arises in the case of individuals equally distributed over both locations. This case is equivalent to the usual random walk. Thus recurrent movement hinders synchronizations between locations. To assess the effect of recurrent movements, we have introduced the commuter ratio which is defined as a ratio of travelling agents and those staying at home. Varying the commuter ratio, we can achieve a retardation of the epidemic in one location with respect to another one.

Furthermore we have considered an epidemic on a one dimensional lattice of locations populated homogeneously (Section 4.4). We have derived corresponding continuum equations. They are structurally different from both the reaction-diffusion counterpart (Fisher, 1937; Kolmogorov et al., 1937) and the continuum version of the direct coupling model (Lopez et al., 1999; Postnikov and Sokolov, 2007). As in the general case (Section 4.2), the continuum direct coupling model is a special case of our approach in the high travel limit. Instead of introduced ad hoc, heuristic coupling strengths of the direct coupling model used in the previous studies (Postnikov and Sokolov, 2007) we derived precise expressions, connecting coupling strengths and travel rates.

In Section 4.4.2, we obtained the analytical expression of the velocity of an epidemic wave front in dependence on travel and infection rates as well as commuter ratio. It is a non-trivial mathematical task, easy tracktable only for the reduced SI model with immobile susceptibles. Our theory predicts saturation of the velocity with increasing parameter values. This stands in contrast to the unbounded square root dependence of the velocity on reaction and travel rates in the reaction-diffusion scheme. Besides the differences mentioned above, numerical integration revealed also similarities between the reaction-diffusion and the recurrent models. For example, at small values of travel rates, the velocity converges extremely slowly towards zero with decreasing travel rate in both the reaction-diffusive and the recurrent cases (Section 4.5). We have shown analytically that the velocity converges towards zero as an inverse logarithm of the travel rate (Section 4.5.2). The results of the agent-based stochastic numerical simulations converge towards our analytical prediction with an increasing number of individuals. Another similarity between the reaction-diffusion and the recurrent model appeared in the range of extremely high infection rates (Section 4.4.5). In this regime contrary to analytical predictions for both models, the velocity grows unboundedly and faster than the square root with the infection rate. This is an artifact of the continuous description of the number of agents. 
Another question of interest closely related to epidemiological applications concerns the shape of the epidemic front. It stands in immediate relation with the front velocity. In the direct coupling case with proper coupling strengths, we obtain that the leading front shape is independent of the infection rate in the SI case or slightly dependent in the SIR and the SIS cases. This stands in contrast to the reaction-diffusion case with strong dependence of the front shape on the infection rate (Section 4.4.6).

Real epidemics evolve on topologies reminiscent of random networks. Therefore, in Section 4.6, we outlined approaches to epidemic spread due to recurrent movements on random networks. These networks consist of nodes representing locations and links representing transportation routes between them. We proposed various plausible models for travel rates, incorporating topological and population disorder. They could serve as a basis for the future research.

Other interesting directions for further exploration include: a generalization to two dimensions, the incorporation of long range interactions, synchronization dynamics in the case of recurrent epidemics (Grenfell et al., 2001; Hagernaas et al., 2004) and explicit assessment of the effective epidemic thresholds . Contacts between individuals always take place in physical space. Thus, our model could account for social heterogeneity (Liljeros et al., 2001; Pastor-Satorras and Vespignani, 2000; Sokolov and Belik, 2003) as well. Our model with recurrent movement or more general models with origin-dependent travel rates and incorporation of migration can also find applications beyond epidemiology. This includes the spread of genes (Novembre et al., 2008) and invasion of species into new habitats known as dispersal (Clobert et al., 2003). Transport of various entities, such as seeds (Nathan, 2006), money bills (Brockmann et al., 2006) or trackable items between geocaches (Brockmann and Theiss, 2008b) should also be elaborated, taking into account the frequently recurrent character of agent movements and rare migration events. Wireless communication is prone to malicious attacks and represents a new threat for various compact mobile devices such as the Blackberry or the iPhone (Kleinberg, 2007). This problem can also be addressed within our general framework. For a successful application of our approach to reality, it is necessary to know the parameters of the system under consideration. In this respect recent advances in empirical assessment of human (González et al., 2008) and animal behavior (Cooke et al., 2004) fill us with hope.

In our work, we unveiled the role of inhomogeneity for long range movements and discovered the novel property of systems with power law distributions in the presence of disorder. We also elucidated the impact of recurrent host movements on epidemic spread, revealing the importance of the proper incorporation of dynamics of host movements into models. We are convinced that our work contributes to a thorough theoretical understanding and appropriate modeling of the spatial epidemic spread in the modern world. 


\section{A Bloch theory}

For dynamics in periodic potentials a very powerful method of Bloch was successfully used in the solid state physics in particularly for calculations of electron conductivity bands in crystal lattices. Eigenstates and eigenvalues of corresponding Hamiltonians are described by what is called the Bloch theory or Flouqet theorem (Ashcroft and Mermin, 1976). We sketch the Bloch theory for our case of generalized topological superdiffusion.

Let us start with the fractional Schrödinger equation (3.4)

$$
\partial_{t} \psi=s^{c-1 / 2} \Delta^{\mu / 2} s^{c-1 / 2} \psi-\psi s^{c-1} \Delta^{\mu / 2} s^{c}=\mathcal{H} \psi,
$$

Performing the separation ansatz $\psi(x, t)=e^{E t} \phi(x)$ we obtain the time independent Schrödinger equation

$$
(\mathcal{H}-E)|\phi\rangle=0 .
$$

Considering our problem in a periodic potential $v(x+2 \pi \lambda)=v(x)$ we assume boundedness and zero average

$$
\frac{1}{2 \pi \lambda} \int_{2 \pi \lambda} d x v(x)=0 \text { and } \frac{1}{2 \pi \lambda} \int_{2 \pi \lambda} d x v^{2}(x)=1 .
$$

It is convenient to consider our problem in the Fourier (impulse) representation $\langle k \mid f\rangle=$ $\mathcal{F}_{k}\{f\}=\tilde{f}=\int d x e^{i k x} f(x)$ :

$$
E \tilde{\phi}(k)-\int d k^{\prime} \tilde{\mathcal{H}}\left(k, k^{\prime}\right) \tilde{\phi}\left(k^{\prime}\right)=0,
$$

with a Hamiltonian

$$
\tilde{\mathcal{H}}\left(k, k^{\prime}\right)=(2 \pi)^{-2} \int d k^{\prime \prime}\left[\tilde{Q}\left(k^{\prime \prime}-k^{\prime}\right)\left|k-k^{\prime \prime}\right|^{\mu} \tilde{R}\left(k-k^{\prime \prime}\right)-\tilde{U}\left(k-k^{\prime}\right)|k|^{\mu} \tilde{U}\left(k^{\prime \prime}-k^{\prime}\right)\right],
$$

where $\tilde{Q}(k)=\mathcal{F}_{k}\left\{s^{c-1}\right\}, \tilde{R}(k)=\mathcal{F}_{k}\left\{s^{c}\right\}, \tilde{U}(k)=\mathcal{F}_{k}\left\{s^{c-1 / 2}\right\}$. Using the Bloch ansatz we look for solutions in the form

$$
\phi(x)=e^{-i q x / \lambda} \theta(x),
$$

where in reduced zone representation the Bloch phase $q$ is restricted to the first Brillouin zone $q \in[0,1]$. The function $\theta(x)$ is periodic with the same period as the potential

$$
\theta(x+2 \pi \lambda)=\theta(x) .
$$

Because of the periodicity Equation (A.4) can be expressed in terms of the Fourier coefficients $\hat{\theta}_{n}=(2 \pi \lambda)^{-1} \int_{2 \pi \lambda} d x \theta(x) e^{-i n x / \lambda}$

$$
\tilde{\phi}(k)=2 \pi \sum_{n} \delta(k+(q-n) / \lambda) \hat{\theta}_{n} .
$$




\section{A Bloch theory}

Analogously expressing $\tilde{Q}, \tilde{R}$ and $\tilde{U}$ through their Fourier coefficients (e.g. $\tilde{U}(k)=2 \pi \sum_{n} \delta(k-$ $\left.k_{n}\right) \hat{U}_{n}$ ), after some cumbersome algebra we arrive at expression

$$
E \tilde{\theta}_{n}-\lambda^{-\mu} \sum_{m, l}\left[\hat{R}_{m-l}|m-l|^{\mu} \hat{Q}_{l-n}-\hat{U}_{m-l}|l-q|^{\mu} \hat{U}_{l-n}\right] \hat{\theta}_{m}=0
$$

Because the last equation is parametrized by the Bloch phase, so are the eigenvalues

$$
\mathcal{H}(q)|\theta\rangle=E(q)|\theta\rangle
$$

which also can be labeled by a discrete index $n$ because they are solutions of the equation (A.5) with periodic boundary conditions. Thus

$$
E=E_{n}(q), \text { with } n \in \mathbb{Z}, q \in[0,1] .
$$

A continuum of eigenvalues labeled by the same index $n$ is called a Bloch band of the Schrödinger equation (A.2). Matrix elements of the Hamiltonian are given through

$$
\langle n|\tilde{\mathcal{H}}(q)| m\rangle=\lambda^{-\mu} \sum_{l}\left[\hat{R}_{m-l}|m-l|^{\mu} \hat{Q}_{l-n}-\hat{U}_{m-l}|l-q|^{\mu} \hat{U}_{l-n}\right] .
$$

In case of vanishing potential $\varepsilon=0$ we have

$$
\langle n|\tilde{\mathcal{H}}(q)| m\rangle=\lambda^{-\mu} \sum_{l}\left[\delta_{m l}|m-l|^{\mu} \delta_{l n}-\delta_{m l}|l-q|^{\mu} \delta_{l n}\right]=-\lambda^{-\mu}|n-q|^{\mu} \delta_{n m}
$$

And thus we recover eigenvalues and eigenfunctions of the free superdiffusive motion

$$
E_{n}^{0}(q)=-\left|\frac{n-q}{\lambda}\right|^{\mu} \quad \text { and } \quad \phi(q)=e^{i(n-q) x / \lambda} .
$$

The band index $n$ and the parameter $q$ together span the whole $\mathbb{R}$ space, and we can denote $k=n-q$ and recover from (A.8) the well-known result for the spectral decomposition of $\Delta^{\mu / 2}: E(k)=-|k|^{\mu}$ and $\phi_{k}(x)=e^{i k x}$ with $k \in \mathbb{R}$. 


\section{B Perturbation theory for random potentials}

The generalized fractional Schrödinger equation reads (3.4)

$$
\mathcal{H} \psi=s^{c-1 / 2} \Delta^{\mu / 2} s^{c-1 / 2} \psi-\psi s^{c-1} \Delta^{\mu / 2} s^{c} \equiv \mathcal{L}_{1} \psi-\mathcal{L}_{2} \psi
$$

Performing the Taylor expansion of the exponential $\left(s=e^{-\varepsilon v}\right)$

$$
\begin{gathered}
s^{c-1 / 2}=e^{-\varepsilon v(c-1 / 2)}=1-(c-1 / 2) \varepsilon v+(c-1 / 2)^{2} \frac{\varepsilon^{2}}{2} v^{2}+\ldots \\
s^{c-1}=e^{-\varepsilon v(c-1)}=1-(c-1) \varepsilon v+(c-1)^{2} \frac{\varepsilon^{2}}{2} v^{2}+\ldots \\
s^{c}=e^{-\varepsilon v c}=1-c \varepsilon v+c^{2} \frac{\varepsilon^{2}}{2} v^{2}+\ldots
\end{gathered}
$$

we obtain up to the second order in $\varepsilon$ :

$$
\begin{gathered}
\mathcal{L}_{1} \psi= \\
\left(1-(c-1 / 2) \varepsilon v+(c-1 / 2)^{2} \frac{\varepsilon^{2}}{2} v^{2}\right) \Delta^{\mu / 2}\left(\psi-(c-1 / 2) \varepsilon v \psi+(c-1 / 2)^{2} \frac{\varepsilon^{2}}{2} v^{2} \psi\right)= \\
\Delta^{\mu / 2} \psi+(c-1 / 2)\left[-\varepsilon\left(\Delta^{\mu / 2} v \psi+v \Delta^{\mu / 2} \psi\right)+\varepsilon^{2}(c-1 / 2)\left(\Delta^{\mu / 2} v^{2} \psi+v \Delta^{\mu / 2} v \psi+\frac{v^{2}}{2} \Delta^{\mu / 2} \psi\right)\right] . \\
\mathcal{L}_{2} \psi= \\
\psi\left(1-(c-1) \varepsilon v+(c-1)^{2} \frac{\varepsilon^{2}}{2} v^{2}\right) \Delta^{\mu / 2}\left(1-c \varepsilon v+c^{2} \frac{\varepsilon^{2}}{2} v^{2}\right)= \\
c \psi\left[-\varepsilon \Delta^{\mu / 2} v+\frac{\varepsilon^{2}}{2}\left(c \Delta^{\mu / 2} v^{2}+2(c-1) v \Delta^{\mu / 2} v\right)\right]
\end{gathered}
$$

(Note that $\Delta^{\mu / 2} 1=0$ ). Let's collect all terms according to their order of $\varepsilon$ together

$$
\begin{aligned}
& \mathcal{H} \psi=\Delta^{\mu / 2} \psi+\varepsilon[c \underbrace{\left\{\Delta^{\mu / 2} v\right\}}_{1}-(c-1 / 2)(\underbrace{\Delta^{\mu / 2} v}_{2}+\underbrace{v \Delta^{\mu / 2}}_{3})] \psi+ \\
& +\frac{\varepsilon^{2}}{2}\left[-c(\underbrace{\left\{\Delta^{\mu / 2} v^{2}\right\}}_{4}+2(c-1) \underbrace{v\left\{\Delta^{\mu / 2} v\right\}}_{5})+(c-1 / 2)^{2}(2 \underbrace{\Delta^{\mu / 2} v^{2}}_{6}+2 \underbrace{v \Delta^{\mu / 2} v}_{7}+\underbrace{v^{2} \Delta^{\mu / 2}}_{8})\right] \psi .
\end{aligned}
$$


So we obtain for the Hamiltonian $\mathcal{H} p=\Delta^{\mu / 2} p+\hat{V}$. The fractional Laplacian $\Delta^{\mu / 2}$ represents the free part and $\hat{V}$ represents the perturbation.

According to the stationary perturbation theory, the eigenvalues up to the second order of $\varepsilon$ are given by (Landau and Lifshitz, 2002)

$$
E_{n}=E_{n}^{0}+V_{n n}+\sum_{m}^{\prime} \frac{\left|V_{m n}\right|^{2}}{E_{n}^{0}-E_{m}^{0}} .
$$

In our case of finite support of length $2 \pi L$, the eigenfunctions of the free Hamiltonian are plane waves, thus matrix elements are calculated according to the formula

$$
V_{n m}=\left\langle\varphi_{n}|\hat{V}| \varphi_{m}\right\rangle=\frac{1}{2 \pi L} \int_{2 \pi L} e^{-i \frac{n}{L} x} \hat{V} e^{i \frac{n}{L} x} d x
$$

The matrix elements corresponding to the numbered terms from Equation (B.7)

$$
\begin{aligned}
\left\langle\varphi_{n}\left|\left\{\Delta^{\mu / 2} v\right\}\right| \varphi_{m}\right\rangle & =-\left|\frac{n-m}{L}\right|^{\mu} \hat{v}_{n-m} \\
\left\langle\varphi_{n}\left|\Delta^{\mu / 2} v \cdot\right| \varphi_{m}\right\rangle & =-\left|\frac{n}{L}\right|^{\mu} \hat{v}_{n-m} \\
\left\langle\varphi_{n}\left|v \Delta^{\mu / 2} \cdot\right| \varphi_{m}\right\rangle & =-\left|\frac{m}{L}\right|^{\mu} \hat{v}_{n-m} \\
\left\langle\varphi_{n}\left|\left\{\Delta^{\mu / 2} v^{2}\right\}\right| \varphi_{m}\right\rangle & =-\left|\frac{n-m}{L}\right|^{\mu}\left(\hat{v}^{2}\right)_{n-m} \\
\left\langle\varphi_{n}\left|v\left\{\Delta^{\mu / 2} v\right\}\right| \varphi_{m}\right\rangle & =-\sum \hat{v}_{n-m-l}\left|\frac{l}{L}\right|^{\mu} \hat{v}_{l} \\
\left\langle\varphi_{n}\left|\Delta^{\mu / 2} v^{2} \cdot\right| \varphi_{m}\right\rangle & =-\left|\frac{n}{L}\right|^{\mu}\left(\hat{v}^{2}\right)_{n-m} \\
\left\langle\varphi_{n}\left|v \Delta^{\mu / 2} v \cdot\right| \varphi_{m}\right\rangle & =-\sum \hat{v}_{n-l}\left|\frac{l}{L}\right|^{\mu} \hat{v}_{l-m} \\
\left\langle\varphi_{n}\left|v^{2} \Delta^{\mu / 2} \cdot\right| \varphi_{m}\right\rangle & =-\left|\frac{m}{L}\right|^{\mu}\left(\hat{v^{2}}\right)_{n-m},
\end{aligned}
$$

we denote by the dot the action of the fractional Laplacian also on the ket vector. Hence up to the second order in $\varepsilon$ follows

$$
\begin{aligned}
V_{n n}=\frac{\varepsilon}{L^{\mu}}(c-1 / 2)\left(|n|^{\mu} \hat{v}_{0}\right. & \left.+|n|^{\mu} \hat{v}_{0}\right)+\frac{\varepsilon^{2}}{2 L^{\mu}}[2 c(c-1) \sum_{l} \underbrace{\hat{v}_{-l}|l|^{\mu} \hat{v}_{l}}_{|l|^{\mu}\left|\hat{v}_{l}\right|^{2}}- \\
& \left.-(c-1 / 2)^{2}(2|n|^{\mu}\left(\hat{v}^{2}\right)_{0}+2 \sum_{l} \underbrace{\hat{v}_{n-l}|l|^{\mu} \hat{v}_{l-n}}_{|l|^{\mu}\left|\hat{v}_{n-l}\right|^{2}}+|n|^{\mu}\left(\hat{v}^{2}\right)_{0})\right]
\end{aligned}
$$




\section{$B$ Perturbation theory for random potentials}

Note that for $\left|V_{m n}\right|^{2}$ we need only the terms of order $\varepsilon$

$$
\begin{aligned}
\left|V_{n m}\right|^{2}=[\varepsilon(\ldots)] \overline{[\varepsilon(\ldots)]} & =\frac{\varepsilon^{2}}{L^{2 \mu}}\left[-c|n-m|_{n-m}^{\mu}+(c-1 / 2)\left(|n|^{\mu}+|m|^{\mu}\right)\right] \times \\
\hat{v}_{n-m} \hat{v}_{m-n} & = \\
& \frac{\varepsilon^{2}}{L^{2 \mu}}\left[-c|n-m|_{n-m}^{\mu}+(c-1 / 2)\left(|n|^{\mu}+|m|^{\mu}\right)\right]^{2}\left|\hat{v}_{n-m}\right|^{2}
\end{aligned}
$$

Thus following the common scheme (B.8) and denoting $k_{n}=n / \lambda, E_{n}^{0}=-\left|k_{n}\right|^{\mu}$ we obtain

$$
\begin{aligned}
& E_{n}=-\left|k_{n}\right|^{\mu}+2 \varepsilon(c-1 / 2)\left|k_{n}\right|^{\mu} \hat{v}_{0}+\frac{\varepsilon^{2}}{2}\left\{2 c(c-1) \sum_{l}\left|k_{l}\right|^{\mu}\left|\hat{v}_{l}\right|^{2}-\right. \\
& -(c-1 / 2)^{2}\left(3 | k _ { n } | ^ { \mu } \left(\hat{\left.\left.v^{2}\right)_{0}+2 \sum_{l}\left|k_{l}\right|^{\mu}\left|\hat{v}_{n-l}\right|^{2}\right)+}\right.\right. \\
& \left.+2 \sum_{m}^{\prime}\left[-c\left|k_{n}-k_{m}\right|^{\mu}+(c-1 / 2)\left|k_{n}\right|^{\mu}+(c-1 / 2)\left|k_{m}\right|^{\mu}\right]^{2} \frac{\left|\hat{v}_{n-m}\right|^{2}}{\left|k_{m}\right|^{\mu}-\left|k_{n}\right|^{\mu}}\right\}
\end{aligned}
$$

Now we introduce the random phase potentials $\hat{v}_{k}=\phi_{k} e^{-i \vartheta_{k}}$ (3.7), for which holds $\left|\hat{v}_{k}\right|^{2}=\phi_{k}^{2}$. We rewrite our sums so that the sum only over positive index appears. Note, that

$$
\sum_{l}\left|k_{l}\right|^{\mu}\left|\hat{v}_{n-l}\right|^{2} \equiv \sum_{l^{\prime}}\left|k_{n}-k_{l^{\prime}}\right|^{\mu} \phi_{l^{\prime}}^{2}
$$

Hence for two underlined terms in (B.12) we obtain

$$
4 c(c-1) \sum_{l>0}\left|k_{l}\right| \phi_{l}^{2}-2(c-1 / 2)^{2} \sum_{l>0}\left[\left|k_{n}-k_{l}\right|^{\mu}+\left|k_{n}+k_{l}\right|^{\mu}\right] \phi_{l}^{2}
$$

Instead of the sum over $m$ in the last term of (B.12) we introduce the new index $l=n-$ $m,(m=n-l)$. Thus for this term holds (note, that $m=-n$ corresponds $l=2 n$ )

$$
\begin{gathered}
\sum_{\substack{l=-\infty \\
l \neq 0,2 n}}^{\infty}\left[c\left|k_{l}\right|^{\mu}+(c-1 / 2)\left|k_{n}\right|^{\mu}+(c-1 / 2)\left|k_{n}-k_{l}\right|^{\mu}\right]^{2} \frac{\phi_{l}^{2}}{\left|k_{n}-k_{l}\right|^{\mu}-\left|k_{n}\right|^{\mu}}= \\
\sum_{l>0}^{\infty} \ldots+\sum_{0<l \neq 2 n}^{\infty} \ldots= \\
\sum_{l>0}\left[c\left|k_{l}\right|^{\mu}+(c-1 / 2)\left|k_{n}\right|^{\mu}+(c-1 / 2)\left|k_{n}+k_{l}\right|^{\mu}\right]^{2} \frac{\phi_{l}^{2}}{\left|k_{n}+k_{l}\right|^{\mu}-\left|k_{n}\right|^{\mu}}+ \\
+\sum_{0<l \neq 2 n}\left[c\left|k_{l}\right|^{\mu}+(c-1 / 2)\left|k_{n}\right|^{\mu}+(c-1 / 2)\left|k_{n}-k_{l}\right|^{\mu}\right]^{2} \frac{\phi_{l}^{2}}{\left|k_{n}-k_{l}\right|^{\mu}-\left|k_{n}\right|^{\mu}} .
\end{gathered}
$$


Thus for the eigenvalues we have the following expression

$$
\begin{aligned}
E_{n}= & -\left|k_{n}\right|^{\mu}+2 \varepsilon(c-1 / 2)\left|k_{n}\right|^{\mu} \hat{\phi}_{0}-\frac{\varepsilon^{2}}{2} 3(c-1 / 2)^{2}\left|k_{n}\right|^{\mu}\left(\widehat{\phi^{2}}\right)_{0}+ \\
& +\frac{\varepsilon^{2}}{2}\left\{4 c(c-1) \sum_{l>0}\left|k_{l}\right|^{\mu} \phi_{l}^{2}-2(c-1 / 2)^{2} \sum_{l>0}\left[\left|k_{n}-k_{l}\right|^{\mu}+\left|k_{n}+k_{l}\right|^{\mu}\right] \phi_{l}^{2}+\right. \\
& +2 \sum_{l>0}\left[c\left|k_{l}\right|^{\mu}+(c-1 / 2)\left|k_{n}\right|^{\mu}+(c-1 / 2)\left|k_{n}+k_{l}\right|^{\mu}\right]^{2} \frac{\phi_{l}^{2}}{\left|k_{n}+k_{l}\right|^{\mu}-\left|k_{n}\right|^{\mu}}+ \\
+ & \left.2 \sum_{0<l \neq 2 n}\left[c\left|k_{l}\right|^{\mu}+(c-1 / 2)\left|k_{n}\right|^{\mu}+(c-1 / 2)\left|k_{n}-k_{l}\right|^{\mu}\right]^{2} \frac{\phi_{l}^{2}}{\left|k_{n}-k_{l}\right|^{\mu}-\left|k_{n}\right|^{\mu}}\right\}
\end{aligned}
$$

Performing the limiting transition $k_{n} \rightarrow k, k_{l} \rightarrow q, 1 / L \rightarrow d k$ from the sum over $l$ to the integral over $q$ we obtain ${ }^{1}$ for the diffusion coefficient $D_{\mu . c}(\varepsilon)=\lim _{k \rightarrow 0}-E(k) / k^{\mu}$

$$
D_{\mu, c}(k ; \varepsilon) / D=1-\varepsilon^{2} G_{\mu, c}(k)
$$

where

$$
G_{\mu, c}(k)=\frac{1}{2 \pi} \int d q S(q) g_{\mu, c}(k / q)
$$

and

$$
\begin{aligned}
g_{\mu, c}(z) & =-2(c-1 / 2)^{2}+\frac{1}{z^{\mu}}\left\{2 c(c-1)-(c-1 / 2)^{2}\left(|1-z|^{\mu}+|1+z|^{\mu}\right)\right. \\
& +\left[(c-1 / 2)\left(z^{\mu}+|1+z|^{\mu}\right)-c\right]^{2} /\left(|1+z|^{\mu}-z^{\mu}\right) \\
& \left.+\left[(c-1 / 2)\left(z^{\mu}+|1-z|^{\mu}\right)-c\right]^{2} /\left(|1-z|^{\mu}-z^{\mu}\right)\right\} .
\end{aligned}
$$
${ }^{1}$ We use here the fact that the term $\left(\widehat{\phi^{2}}\right)_{0}$ is nothing else as $\int v^{2}(x) d x \equiv \int \hat{v}^{2}(\kappa) d \kappa=\frac{1}{2 \pi L} \int S(\kappa) d \kappa$. Also
$\hat{v}_{0}=\int v(x) d x=0$ because we consider only potentials with zero mean. 


\section{Acknowledgments}

I dedicated this thesis to my wonderful mother Nionila Belik who suddenly passed away during my $\mathrm{PhD}$ studies. She supported all my decisions during the very short 25 years. I am very thankful to her for always considering me an independent person and I am obliged to her for all my best qualities. The second person, whom I dedicated this thesis, is my devoted grandmother Praskovia. In her garden, I spent very nice summers and had very pleasant time reading a lot of books. Ways of my life were paved there.

I would like to express my gratitude to Theo Geisel for giving me the opportunity to work on my PhD in the great and very creative environment of our department, and for allowing me to give the two-days talk in the Winterseminar.

I would like to thank Dirk Brockmann for drawing my attention to topics elaborated in this thesis, for encouraging me to work independently and to look into the essence.

I would like to thank Alejandro Morales Gallardo, Benjamin Schwenker, Vincent David, Rafael Brune and Christian Thiemann for the work on epidemics together.

I would like to thank Kai Bröking for a lot of help and for providing me with books and DVDs. I would like also to thank him, together with Andreas Sorge and Peter Hiemeyer, for being nice roommates.

I want to thank all other members of our department, in particular: Oliver Bendix, Dmitry Bibichkov, Hecke Degering, Harold Gutsch, Katja Fiedler, Ragnar Fleischmann, Denny Fliegner, Tanja Gindele, Joachim Haß, Holger Hennig, Frank Hesse, Moritz Hiller, Min Huang, Katharina Jeremias, Wolfgang Keil, Tsampikos Kottos, Georg Martius, Raoul Martin Memmesheimer, Jakob Metzger, Björn Naundorf, Tobias Niemann, Lars Reichl, Michael Schnabel, Tatjana Tchumatchenko, Marc Timme, Corinna Trautsch, Fred Wolf and Regina Wunderlich for contributing to the unique atmosphere of our institute.

I want to thank Lars Hufnagel, Olga A. Chichigina, and Igor M. Sokolov for valuable discussions - not only about science - and support, especially at the beginning of my $\mathrm{PhD}$.

Tatiana Engel, Anna Levina, Anastasiya Lavrova, Tilo Schwalger, Wolfgang Pietsch, Vasily Zaburdaev and Robert Engel I want to thank for many funny hours spent together and for careful proofreading of the manuscript. I acknowledge the friendly support by Ira Schwalger, Katya Zhuchkova, David Strehober, Larisa Zaburdaeva, Oxana Actis and Xenia Fomenko.

I would like to thank family Belikov - my uncle Vladimir, my aunt Galina and my cousin Svetlana - for just being there.

I also would like to thank family Loskutov, my best friends in Moscow, especially, Margarita and Vladimir for helping me to manage a lot of troubles.

I want to thank Kunibert and Ingeborg Kraski for being so kind to me as if they were my parents. Families Mehner and Weiser I want to thank for accepting me as one of the family.

My daughter Nila Johanna I thank for her nice character and for providing me with inspirition and hope. 
Last but not least, I want to thank my best friend and wife Gitte, for indispensable support during the last years, for sharing good and bad times with me, and having the patience to commute between Berlin and Göttingen. 


\section{Bibliography}

Albert, R. and Barabási, A. L. (2002). Statistical mechanics of complex networks. Reviews of Modern Physics, 74:47.

Anderson, P. W. (1958). Absence of diffusion in certain random lattices. Physical Review, 109:1492.

Anderson, R. M. and May, R. M. (1991). Infectious diseases of humans. Oxford university press.

Arino, J. and van den Driessche, P. (2003). Positive Systems, chapter The Basic Reproduction Number in a Multi-city Compartmental Epidemic Model. Springer-Verlag, Berlin.

Ashcroft, N. W. and Mermin, N. D. (1976). Solid state physics. Saunders College Publishing.

Astumian, R. D. and Bier, M. (1994). Fluctuation driven ratchets: Molecular motors. Physical Review Letters, 72(1766).

Bachelier, L. (1900). Théorie de la spéculation. PhD thesis, Faculty of Sciences of the Academy of Paris.

Belik, V. V. and Brockmann, D. (2007). Accelerating random walks by disorder. New Journal of Physics, 9:54.

ben Avraham, D. and Havlin, S. (2000). Diffusion and reaction in fractals and disordered systems. Cambridge university press.

Bénichou, O., Coppey, M., Moreau, M., Suet, P.-H., and Voituriez, R. (2005). Optimal search strategies for hidden targets. Physical Review Letters, 94:198101.

Boccaletti, S., Latora, V., Moreno, Y., Chavez, M., and Hwang, D. (2006). Complex networks: stucture and dynamics. Physics Reports, 424:175.

Bouchaud, J.-P. and Georges, A. (1990). Anomalous diffusion in disordered media: statistical mechanism, models and physical applications. Physics Reports, 195:127.

Bramson, M. (1983). Convergence of solutions of the kolmogorov non-linear diffusion equations to travelling waves. Memoirs of the American Mathematical Society, 44:285.

Brockmann, D. (2003). Superdiffusion in scale-free inhomogeneous environments. PhD thesis, Georg-August-Universität zu Göttingen. 
Brockmann, D. and Geisel, T. (2000). The ecology of gaze shifts. Neurocomputing, 32-33:643.

Brockmann, D. and Geisel, T. (2003a). Lévy flights in inhomogeneous media. Physical Review Letters, 90:170601.

Brockmann, D. and Geisel, T. (2003b). Particle dispersion on rapidly folding random heteropolymers. Physical Review Letters, 91:048303.

Brockmann, D. and Hufnagel, L. (2007). Front propagation in reaction-superdiffusion dynamics: Taming Lévy flights with fluctuations. Physical Review Letters, 98:178301.

Brockmann, D., Hufnagel, L., and Geisel, T. (2006). The scaling laws of human travel. Nature (London), 439:462.

Brockmann, D. and Sokolov, I. M. (2002). Lévy flights in external force fields: from models to equations. Chemical Physics, 284:409.

Brockmann, D. and Theiss, F. (2008b). Money circulation, trackable items, and the emergence of universal human mobility patterns. Pervasive Computing, 7:28.

Brown, R. (1866). The miscellaneous botanical works of Robert Brown, volume 1. R. Hardwicke, London.

Brunet, E. and Derrida, B. (1997). Shift in the velocity of a front due to a cutoff. Physical Review E, 56:2597.

Camitz, M. and Liljeros, F. (2006). The effect of travel restrictions on the spread of a moderately contageous disease. BMC Medicine, 4:32.

Chen, L. and Deem, M. W. (2003). Two-dimensional diffusion in the presence of topological disorder. Physical Review E, 68:021107.

Cliff, A. and Haggett, P. (2004). Time, travel and infection. British Medical Bullitin, 69:87-99.

Clobert, J., Danchin, E., Dhondt, A. A., and Nichols, J. D., editors (2003). Dispersal. Oxford university press.

Collizza, V., Pastor-Satorras, R., and Vespignani, A. (2007). Reaction-diffusion processes and metapopulation moldels in heterogeneous networks. Nature Physics.

Condamin, S., Bénichou, O., Tejedor, V., Voituriez, R., and Klafter, J. (2008). First-passage times in complex scale-invariant media. Nature (London), 450:77.

Cooke, S. J., Hinch, S. G., Wikelski, M., Andrews, R. D., Kuchel, L. J., Wolcott, T. G., and J.Butler, P. (2004). Biotelemetry: a mechanistic approach to ecology. Trends in Ecology and Evolution, 19:334.

Daley, D. J. and Gani, J. (1999). Epidemic Modelling. Cambridge university press. 
David, V. and Brockmann, D. (2009). in preparation.

de L Kronig, R. and Penney, W. G. (1931). Quantum mechanics of electrons in crystal lattices. Proceedings of the Royal Society of Science A, 130:499.

Diekmann, O. and Heesterbeek, J. A. P. (2000). Mathematical Epidemiology of Infectious Diseases. Wiley, New Yorck.

Doi, M. (1976a). Second quantization representation for classical many-particle system. Journal of Physics A: Mathematical and General, 9:1465.

Doi, M. (1976b). Stochastic theory of diffusion-controlled reactions. Journal of Physics A: Mathematical and General, 9:1479.

Dybiec, B., Gudowska-Nowak, E., and Sokolov, I. M. (2008). Transport in a Lévy ratchet: Group velocity and distribution spread. Physical Review E, 78:011117.

Ebeling, W., Gudowska-Nowak, E., and Sokolov, I. M. (2008). On stochastic dynamics in physics — remarks on history and terminology. Acta Physica Polonica B, 39:1003.

Ebeling, W. and Sokolov, I. M. (2006). Statistical thermodinamics and stochastic theory of nonequilibrium systems. World Scientific, Singapur.

Economou, E. N. (2006). Green's Functions in Quantum Physics. Springer-Verlag, Berlin.

Edwards, A. M., Phillips, R. A., Watkins, N. W., Freeman, M. P., Murphy, E. J., Afanasyev, V., Buldyrev, S. V., da Luz, M. G. E., Raposo, E. P., Stanley, H. E., and Viswanathan, G. M. (2008). Revisiting lévy flight search patterns of wandering albatrosses, bumblebees and deer. Nature (London), 449:1044.

Einstein, A. (1905). Über die von molekularkinetischen Theorie der Wärme geforderte Bewegung von in ruhenden Flüssigkeiten suspendierten Teilchen. Annalen der Physik, 17:549.

Erlander, S. (1980). Optimal spatial interaction and the gravity model. Lecture notes in Economics and mathematical systems. Springer-Verlag, Berlin.

Eubank, S., Guclu, H., Kumar, V. S. A., Marathe, M. V., Srinivasan, A., Toroczkai, Z., and Wang, N. (2004). Modelling disease outbreaks in realistic urban social networks. Nature (London), 429:180.

Feller, W. (1971). An introduction to probability theory and its application, volume 1, 2. Wiley, New Yorck.

Ferrari, M. J., Grais, R. F., Bharti, N., Conlan, A. J. K., Bjørnstad, O. N., Wolfson, L. J., Guerin, P. J., Djibo, A., and Grenfell, B. T. (2008). The dynamics of measles in sub-Saharan Africa. Nature (London), 451:679.

Feynman, R. P., Leighton, R. B., and Sands, M. (1966). The Feynman Lectures in Physics, volume 1. Addison-Wesley, Reading, MA. 
Fick, A. (1855). Ueber Diffusion. Annalen der Physik, 170:59.

Fisher, R. A. (1937). The wave of advance of advantageous gene. Annals of Eugenics, 7:355369.

Fogedby, H. C. (1998). Lévy flights in quenched random force fields. Physical Review E, 58:1690.

Gardiner, G. W. (1997). Handbook of stochastic methods. Springer-Verlag, Berlin.

Garrothers, G. A. P. (1956). An historical review of the gravity and potential concepts of human interaction. Journal of the American Planning Association, 22:94.

Gastner, M. T. and Newman, M. E. J. (2006). The spatial structure of networks. European Physical Journal B, 49:247.

Geisel, T. (1985). Accelerated Diffusion in Josephson Junctions and Related Chaotic Systems. Phys. Rev. Lett., 54:616.

González, M. C., Hidalgo, C. A., and Barabási, A.-L. (2008). Understanding individual human mobility patterns. Nature (London), 453:779.

Grenfell, B. T., Bjornstad, O. N., and Kappey, J. (2001). Travelling waves and spatial hierarchies in measles epidemics. Nature (London), 414:716.

Hagernaas, T. J., Donnelly, C. A., and Ferguson, N. M. (2004). Spatial heterogeneity and the percictence of the infectious diseases. Journal of Theoretical Biology, 229:349.

Hairer, E., Nørsett, S. P., and Wanner, G. (2000). Solving ordinary differential equations I. Springer-Verlag, Berlin.

Hairer, E., Nørsett, S. P., and Wanner, G. (2002). Solving ordinary differential equations II. Springer-Verlag, Berlin.

Hanski, I. (1998). Metapopulation dynamics. Nature (London), 396:41.

Haus, J. W. and Kehr, K. W. (1987). Diffusion in regular and disordered lattices. Physics Reports, 150:263.

Helbing, D. (1997). Verkehrsdynamik. Springer-Verlag, Berlin.

Helbing, D., Farkas, I., and Vicsek, T. (2000). Simulating dynamical features of escape panic. Nature (London), 407:487.

Hilfer, R., editor (2000). Application of fractional calculus in physics. World Scientific, Singapur.

Holland, M. D. and Hastings, A. (2008). Strong effect of dispersal network structure on ecological dynamics. Nature (London). 
Honerkamp, J. (1990). Stochastische dynamische Systeme. VCH Verlaggesellschaft mbH, Weinheim.

Horsthemke, W. (1999). Fisher waves in reaction random walks. Physics Letters A, 263:285.

Horsthemke, W. and Lefever, R. (2006). Noise-Induced Transitions. Springer Series in Synergetics. Springer-Verlag, Berlin.

Hufnagel, L., Brockmann, D., and Geisel, T. (2004). Forecast and control of epidemics in a globalized world. Proceedings of the National Academy of Sciences of the United States of America, 101:15124.

Hughes, B. D. (1995b). Random walks and Random Environments, volume 2. Oxford university press.

Jung, W.-S., Wang, F., and Stanley, H. E. (2008). Gravity model in the korean highway. European Physics Letters, 81:48005.

Keeling, M. J. and Rohani, P. (2005). Estimating spatial coupling in epidemiological systems: a mechanistic approach. Ecology Letters, 5:20-29.

Keeling, M. J. and Rohani, P. (2007). Modeling infectious diseases. Princeton University Press.

Kermack, W. O. and McKendrick, A. G. (1927). A contribution to the mathematical theory of epidemics. Proceedings of the Royal Society of London, Series A, 115:700.

Kessler, D. A., Ner, Z., and Sander, L. M. (1998). Front propagation: Precursors, cutoffs, and structural stability. Physical Review E, 58:107.

Kleinberg, J. (2007). The wireless epidemic. Nature (London), 449:287.

Kolmogorov, A., Petrovsky, I., and Piscounov, N. (1937). Étude de l'équation de diffusion avec croissance de la quantité de matière et son application à un problème biologique. Bulletin de l'université d'état à Moscou, Série internationale, section A, 1:1.

Kozma, B., Hastings, M. B., and Korniss, G. (2005). Diffusion processes on power-law smallworld networks. Physical Review Letters, 95:018701.

Kree, R., Schaub, B., and Schmittman, B. (1989). Effects of pollution on critical population dynamics. Physical Review A, 39(2214).

Kuzovkov, V. and Kotomin, E. (1988). Kinetics of bimolecular reactions in condensed media: critical phenomena and microscopic self-organisation. Reports on Progress in Physics, 51:1479.

Landau, L. D. and Lifshitz, E. M. (2002). Quantum Mechanics: Non-Relativistic Theory. Butterworth-Heinemann College. 
Lee, B. P. (1994). Renormalization group calculation for the reaction kA to OE. Journal of Physics A: Mathematical and General, 27:2633.

Lévy, P. (1954). Théorie de l'Addition des Variables Alèatoires. Gauthier-Villars, Paris.

Liljeros, F., Edling, C. R., Amaral, L. A. N., Stanley, H. E., and Åberg, T. (2001). Promiscuous individuals are the vulnerable nodes to target safe-sex campaigns. Nature (London), 411:907-908.

Lomholt, M. A., Ambjornsson, T., and Metzler, R. (2005). Optimal target search on a fastfolding polymer chain with volume exchange. Physical Review Letters, 95:260603.

López, E., Parshani, R., Cohen, R., Carmi, S., and Havlin, S. (2007). Limited path perolation in complex networks. Physical Review Letters, 99:188701.

Lopez, L. F., Coutinho, F. A. B., Burattini, M. N., and Massad, E. (1999). Modelling the spread of infections when the contact rate among individuals is short ranged: Propagation of epidemic waves. Mathematical and Computer Modelling, 29:55.

Mai, J., Sokolov, I. M., and Blumen, A. (2000). Front propagation in one-dimensional autocatalytic reactions: The breakdown of the classical picture at small particle concentrations. Phys. Rev. E, 62:141.

May, R. M. and Lloyd, A. L. (2001). Infection dynamics on scale-free networks. Physical Review Letters, 64:066112.

Méndez, V., Campos, D., and Fedotov, S. (2004). Front propagation in reaction-dispersal models with finite jump speed. Physical Review E, 70:036121.

Metzler, R. and Klafter, J. (2000). The random walk's guide to anomalous diffusion: a fractional dynamics approach. Phys. Rep., 339:1.

Metzler, R. and Klafter, J. (2004). The restaurant at the end of the random walk: recent developments in the description of anomalous transport by fractional dynamics. J.Phys. A: Math. Gen., 37:R161.

Montroll, W. and Shlesinger, M. (1984). On the wonderful world of random walks, volume 11 of Studies in statistical mechanics. North-Holland, Amsterdam.

Murray, J. D. (1993). Mathematical biology. Springer-Verlag, Berlin.

Naether, U., Postnikov, E. B., and Sokolov, I. M. (2008). Infection fronts in contact disease spread. The European Physical Journal B, 65:353.

Nathan, R. (2006). Long-Distance Dispersal of Plants. Science, 313(5788):786-788.

Newman, M. E. J. (2002). Spread of epidemic disease on networks. Physical Review E, 66:016128. 
Newman, M. E. J. (2006). Modularity and community structure in networks. Proceedings of the National Academy of Sciences, 103:8577.

Noble, J. V. (1974). Geographic and temporal development of plagues. Nature (London), 250:726.

Nolting, W. (2000). Grundkurs Theoretische Physik, volume 5. Vieweg (Braunschweig/Wisbaden).

Novembre, J., Johnson, T., Bryc, K., Kutalik, Z., Boyko, A. R., Auton, A., Indap, A., King, K. S., Bergmann, S., Nelson, M. R., Stephens, M., and Bustamante, C. D. (2008). Genes mirror geography within europe. Nature, 449:98.

Nowak, M. A. (2006). Evolutionary dynamics. The Belknap Press of Harvard University Press.

Panja, D. (2004). Effects of fluctuations on propagating fronts. Physics Reports, 393:87.

Parham, P. E. and Ferguson, N. M. (2006). Space and contact networks: capturing the locality of disease transmission. Journal of the Royal Society Interface, 3:483.

Pastor-Satorras, R. and Vespignani, A. (2000). Epidemic spreading in scale-free networks. Physical Review Letters, 86:3200.

Pechenik, L. and Levine, H. (1999). Interfacial velocity corrections due to multiplicative noise. Physical Review E, 59:3893.

Porta, A. L., Voth, G. A., Crawford, A. M., Alexander, J., and Bodenschatz, E. (2001). Nature (London), 409:1017.

Postnikov, E. B. and Sokolov, I. M. (2007). Continuum description of a contact infection spread in a SIR model. Mathematical Biosciences, 208:205.

Raposo, E. P., Buldyrev, S. V., da Luz, M. G. E., Santos, M. C., Stanley, H. E., and Viswanathan, G. M. (2003). Dynamical robustness of lévy search strategies. Physical Review Letters, 91:240601.

Reichenbach, T., Mobilia, M., and Frey, E. (2007). Mobility promotes and jeopardizes biodiversity in rock-paper-scissors games. Nature, 448(7157):1046-1049.

Reluga, T. C., Medlock, J., and Galvani, A. P. (2006). A model of spatial epidemic spread when individuals move within overlapping home ranges. Bulletin of Mathematical Biology, 68:401.

Riley, S. (2007). Large-scale spatial-transmission models of infectious disease. Science, 316:1298.

Risken, H. (1996). The Fokker-Planck equation. Springer-Verlag, Berlin. 
Rushton, S. and Mautner, A. J. (1955). The deterministic model of a simple epidemic for more than one community. Biometrika, 42:126-132.

Russell, C. A., Jones, T. C., Barr, I. G., Cox, N. J., Garten, R. J., Gregory, V., Gust, I. D., Hampson, A. W., Hay, A. J., Hurt, A. C., de Jong, J. C., Kelso, A., Klimov, A. I., Kageyama, T., Komadina, N., Lapedes, A. S., Lin, Y. P., Mosterin, A., Obuchi, M., Odagiri, T., Osterhaus, A. D. M. E., Rimmelzwaan, G. F., Shaw, M. W., Skepner, E., Stohr, K., Tashiro, M., Fouchier, R. A. M., and Smith, D. J. (2008). The Global Circulation of Seasonal Influenza A (H3N2) Viruses. Science, 320:340.

Rvachev, L. A. and Longini, I. M. (1985). A mathematical model for the global spread of influenza. Mathematical Biosciences, 75:3.

Sattenspiel, L. and Dietz, K. (1995). A sructured epidemic model incorporating geographic mobility among regions. Mathematical Biosciences, 128:71.

Schwenker, B. (2008). Super fast epidemic spread. Master's thesis, Georg-August-Universität zu Göttingen.

Sims, D. W., Southall, E. J., Humphries, N. E., Hays, G. C., Bradshaw, C. J. A., Pitchford, J. W., James, A., Ahmed, M. Z., Brierley, A. S., Hindell, M. A., Morritt, D., Musyl, M. K., Righton, D., Shepard, E. L. C., Wearmouth, V. J., Wilson, R. P., Witt, M. J., and Metcalfe, J. D. (2008). Scaling laws of marine predator search behaviour. Nature (London), 451:1098.

Sokolov, I. M. (2000). Lévy flights from a continuous-time process. Phys. Rev. E, 63:011104.

Sokolov, I. M. and Belik, V. V. (2003). Competition between lévy flights and continuoius drift. Physica A, 330:46.

Sokolov, I. M., Metzler, R., Pant, K., and Williams, M. C. (2005). Target search of n sliding proteins on a dna. Biophysical Journal, 89:895.

Stoer, J. and Bulirsch, R. (2002). Introduction to numerical analysis. Springer-Verlag, Berlin.

Stollenwerk, N. and Jansen, V. A. A. (2007). Complex population dynamics, chapter Criticality in epidemiology, page 159. World Scientific, Singapur.

Stratonovich, R. L. (1963). Topics in the Theory of Random Noise. Gordon and Breach, New York.

Tatem, A. J., Hay, S. I., and Rogers, D. J. (2006). Global traffic and disease vector dispersal. Proceedings of the National Academy of Sciences of the United States of America, 103:6242.

Toroczkai, Z., Kozma, B., Bassler, K. E., Hengartner, N. W., and Korniss, G. (2008). Gradient networks. Journal of Physics A: Mathematical and Theoretical, 41:155103.

van Kampen, N. G. (2007). Stochastic Processes in Chemistry and Physics. North-Holland, Amsterdam. 
Viboud, C., Bjørnstad, O. N., Smith, D. L., Simonsen, L., Miller, M. A., and Grenfell, B. T. (2006). Synchrony, waves, and spatial hierarchies in the spread of influenza. Science, $312: 447$.

Viswanathan, G. M., Buldyrev, S. V., Havlin, S., da Luz, M. E. G., Raposo, E. P., and Stanley, H. E. (1999a). Lévy flight search patterns of wandering albatrosses. Nature (London), 401:911.

Viswanathan, G. M., Buldyrev, S. V., Havlin, S., da Luz, M. G. E., Raposo, E. P., and Stanley, H. E. (1999b). Optimizing the success of random searches. Nature (London), 401:911.

Volterra, V. (1926). Fluctuations in the abundance of a species considered mathematically. Nature (London), 118:558.

Watts, D. J., Muhamad, R., Muhamad, M., Daniel, C., and Dodds, P. S. (2005). Multiscale, resurgent epidemics in a hierarchical metapopulation model. Proceedings of the National Academy of Sciences of the United States of America, 102:11157.

Watts, D. J. and Strogatz, S. H. (1998). Collective dynamics of 'small-world' networks. Nature (London), 393:440.

Wilson, A. G. (1967). A statistical theory of spatial distribution modes. Transportation Research Part A, 1:253.

Winkle, S. (2005). Geißeln der Menschheit: Die Kulturgeschichte der Seuchen. Artemis \& Winkler.

Xia, Y., Bjørnstad, O. N., and Grenfell, B. T. (2004). Measles metapopulation dynamics: A gravity model for epidemiological coupling and dynamics. The American Naturalist, 164:267.

Zaburdaev, V., Schmiedeberg, M., and Stark, H. (2008). Random walks with random velocities. Physical Review E, 78:011119.

Zipf, G. K. (1946). The P1 P2/D Hypothesis: On the Intercity Movement of Persons. American Sociological Review, 11:677. 